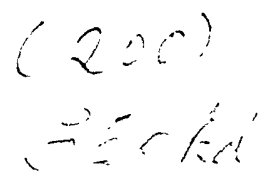

UNITED STATES

DEPARTMENT OF THE INTERIOR

GEOLOGICAL SURVEY

Ground Water Branch

\title{
DATA ON WATER WELLS IN THE \\ FREMONT VALLEY AREA, \\ KERN COUNTY, CALIFORNIA
}

By

L. C. Dutcher

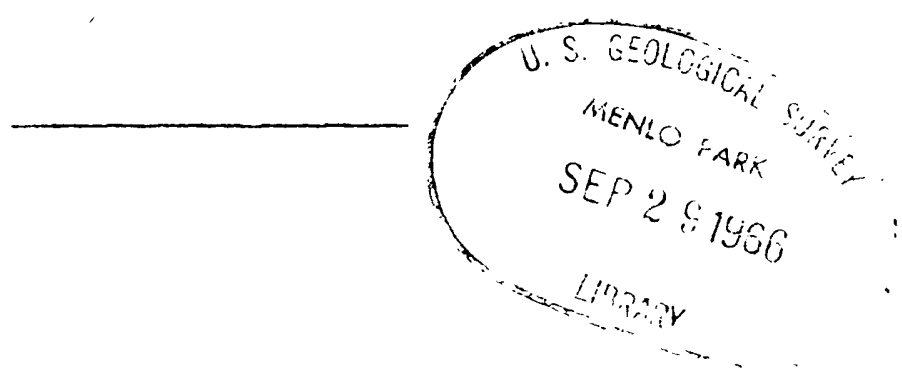

Prepared in cooperation with the California Department of Water Resources

OPEN-FILE REPORT

$$
56.35
$$

Long Beach, California

1959 
CONTENTS

Page

Purpose and scope of the work and report

Location and general features of the area... 5

Previous investigations and acknowledgments 7

Geologic and hydrologic features of the area

Economic aspects of ground water in the area... 14

Well-numbering system-15

References c1ted... 17

TABLES

Table 1. Descriptions of wells in the Fremont Valley area,

California-..... 20

2. Cross index of other well numbers and Geological

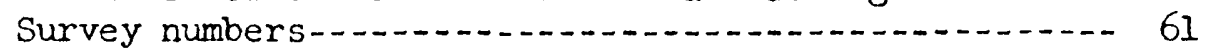

Part 1. Numbers used by Thompson (1929) ...... 61

2. Numbers used by Cyril Williams, Jr.,

(1930)

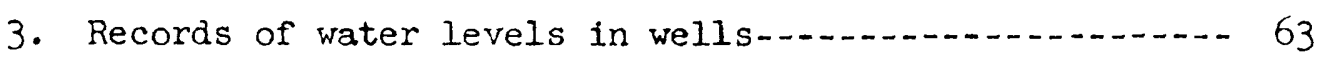

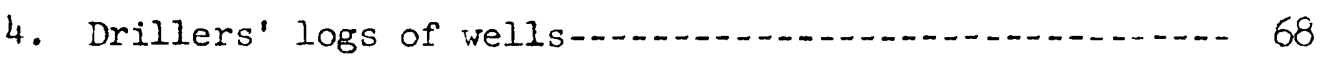

5. Chemical analyses of water from wells

\section{PLATE}

Plate 1. Map of the Fremont Valley area, California, showing reconnaissance geology and locations of wells-.....-In pocket 
DATA ON WATER WEILS IN THE FREMONT VALLEY AREA, KERN COUNTY, CALIFORNIA

By L. C. Dutcher

PURPOSE AND SCOPE OF THE WORK AND REPORT

The data presented in this tabulation were collected by the U. S. Geological Survey in connection with an investigation of water wells and general hydrologic conditions throughout much of the desert region of southern California. The geologic mapping was financed by Federal funds for arid-regions studies, and the canvass of wells and compilation of data were financed under a cooperative agreement with the California Department of Water Resources.

The desert regions of California are characterized by barren mountain ranges and isolated hills surrounding broad valleys or basins which are underlain by alluvial debris derived from the surrounding highlands. These basins generally contain ground water which has a wide range in chemical quality and which can be and in some areas has been developed for beneficial use.

The general objective of the cooperative investigation is to collect and to tabulate all available hydrologic data for the individual desert basins in order to provide public agencies and the general public with data for use in planning water utilization and management and for use in possible subsequent ground-water investigations. 
Accordingly, the scope of the work carried out by the Geological Survey in each area has included: (1) Brief reconnaissance mapping of major geologic features to define the extent and general character of the deposits that contain the ground water; (2) visiting and examining virtually all the water wells in the area; determining and recording their locations in relation to geograpinic and cultural features and the public-land net, wherever possible; and recording well depths and sizes, types and capacities of installed equipment, uses of the water, and other pertinent information available at the well site; (3) measurement of the depth to the water surface below an established and described measuring point at or near the land surface; (4) selection of representative wells to be measured periodically in order to detect and record changes of water levels; and (5) collection and assembly of well records, Including well logs, water-level measurements, and chemical analyses. The work has been carried on by the U. S. Geological Survey under the general supervision of Harry D. Wilson, Jr., district engineer in charge of ground-water investigations in California, and under the immediate supervision of Fred Kunkel, geologist in charge of the Long Beach subdistrict office. 
LOCATION AND GENERAL FEATURES OF THE AREA

The Fremont Valley area covers about 680 square miles and includes most of Fremont Valley and the northeastern part of Antelope Valley as defined by Thompson (1929, pls. 16 and 19) and parts of the Tehachapi, El Paso, and Rand Mountains (pl. 1).

The area of this study lies in the southwestern part of the Mojave Desert region between $117^{\circ} 35^{\prime}$ and $118^{\circ} 10^{\prime}$ west longitude and about $35^{\circ} 00^{\prime}$ and $35^{\circ} 25^{\prime}$ north latitude, north and east of the town of Mojave. The eastern boundary of the area coincides with the Kern and San Bernardino Counties' boundary line at the west edge of the Cuddeback, Superfor, and Harper Valleys (Kunkel, 1956); the southern boundary in the Antelope Valley is U. S. Highway 466 (Edwards Air Force Base); and in the Fremont Valley is the Muroc fault at the northeast edge of the Willow Springs, Gloster, and Chaffee areas (Kunkel and others, 1957); the western boundary is the Sierra Nevada; and the northern boundary is the El Paso Mountains.

The area includes one large ground-water basin in Fremont Valley, part of the North Muroc basin north of U. S. Highway 466 in Antelope Valley, and three or more minor basins or subbasins in Antelope Valley in the area east of Castle Butte and south of the Rand Mountains. The large basin in Fremont Valley extends north from Castle Butte to the Sierra Nevada and El Paso Mountains and on the northeast is contained in the narrow depression between the $E I$ Paso and Rand Mountains. 
The area extending from the Rand Mountains southward to North Muroc basin is, in general, uplifted in relation to the main valley area to the north and west. Granitic rocks (bedrock), volcanic rocks, and virtually impermeable sedimentary rocks of Tertiary age crop out in many hills and in extensive areas of low relief. Many faults strike across the area, and ground water occurs in the small but locally deep depressions bounded by these geologic structures or by hills of impermeable rock.

The surface drainage of the area is of the closed type, and infrequent munoff reaches one or another of the small playas shown on plate 1, or reaches the larger playas known as Koehn Lake, in the northeastern part of Fremont Valley, and Rogers Lake (south of U. S. Highway 466, and not shown on pl. 1) in the northeastern part of Antelope Valley.

The area is shown on all or parts of the following U. S. Geological Survey topographic quadrangle maps: Boron, Castle Butte, Cross Mountain, Kramer, Mojave, Randsburg, and Saltdale, all at a scale of 1:62,500. Access to the area is provided by U. S. Highways 6 and 466 and several paved and many unpaved roads.

Geographically the area consists mainly of alluvial fans and plains built out from the Sierra Nevada, El Paso, and Rand Mountains. On the east and south the Rosamond Hills, Castle Butte, Desert Butte, and other isolated hills rise above the valley floor. 


\section{PREVIOUS INVESTIGATIOINS AND ACKNOWLEDGMENTS}

Data on ground water in the Fremont Valley area are contained in three reports: U. S. Geological Survey Water-Supply Paper 578, "The Mohave Desert Region, California" (Thompson, 1929, p. 201-223, 289-371), includes data obtained in 1918 on 53 wells in the area of the present investigation; a U. S. Geological Survey open-file report, "Ground-Water Reconnaissance in the Western Part of the Mojave Desert, Calif., with Particular Respect to the Boron Content of Well Water" (Stone, 1957), contains data collected in 1954 on 171 wells in the area; and a private report (Williams, 1930) contains information collected in 1929 and 1930 on 23 wells in the area. The data on wells from all these reports are included herein.

Part of Fremont Valley and viclnity is shown on the geologic maps of the Saltdale and Castle Butte quadrangles by Dibblee (1952 and 1958). The geology shown on plate 1 was compiled and modified from the published maps mentioned above and from unpublished maps of the Boron and Mojave quadrangles by T. W. Dibolee and unpublished maps by the author.

The California Department of Water Resources provided access to all pertinent information in its files, including numerous well logs and chemical analyses. The U. S. Borax and Chemical Corp. and the $M$ and $R$ Sheep and Cattle Co. provided access to a large amount of data in their files, as did many private well owners, well drillers, and others. The cooperation and assistance given by these people and agencies contributed materially to the completeness of the data presented in this report, and are most gratefully acknowledged. 


\section{GEOLOGIC AND HYDROLOGIC FEATURES OF THE AREA}

The geologic units in Fremont Valley and vicinity can be grouped into two broad categories: Consolidated rocks and unconsolidated deposits. The consolidated rocks are for the most part impervious and, except for minor amounts of water in cracks and weathered zones, yield little or no water. The consolidated rocks (pl. 1) comprise the old crystalline, metamorphic, and consolidated sedimentary rocks of pre-Tertiary age which collectively form the basement complex (map symbol $\mathrm{bc}$ ) and the consolidated sedimentary rocks of Tertiary age (map symbol $\underline{\mathrm{Tc}}$ ).

The consolidated sedimentary and pyroclastic rocks of Tertiary age (map symbol $\mathrm{Tc}$ ) are part of the Goler formation of Eocene to early Miocene age and the Ricardo formation of Pliocene age mapped by Dibblee (1952) in the Saltdale quadrangle, and the Tropico group of Miocene(?) and Pliocene(?) age mapped by Dibblee (1958) in the Castle Butte quadrangle. They consist mainly of gray and red conglomerate, arkose, cobble gravel, tuff, sandstone, chert, limestone, gravel, sand, silt, and clay. For the most part these rocks are poorly permeable, but locally where penetrated by deep wells they yield small amounts of water to domestic wells.

Volcanic rocks of acidic composition, mainly andesite, rhyolite, and dacite of Miocene and Pliocene age (map symbol Tav), also occur in the Fremont Valley area. These rocks are part of the Ricardo formation mapped by Dibblee (1952) in the Saltdale quadrangle and the Tropico group mapped by Dibblee (1958) in the Castle Butte quadrangle. 
Extrusive and intrusive basalts of Miocene(?) to Pleistocene age (map symbol QTb) also occur in the area. These rocks are part of the Ricardo formation mapped by Dibblee (1952) in the Saltdale quadrangle, the Tropico group mapped by Dibblee (1958) in the Castle Butte quadrangle, and the Black Mountain basalt mapped in the Saltdale quadrangle by Dibblee (1952).

The unconsolidated older alluvium of late Pleistocene age (map symbol Qoal) consists of compact arkosic gravel, sand, silt, and clay. The deposits are weathered, and locally the feldspars have been altered to clay. Near the hills the unit is predominantly gravel but beneath the valley areas it is finer grained and better sorted. Because the older alluvium overlies older fan deposits (Qof) or Tertiary continental rocks ( $\mathrm{Tc}$ ) on which an erosional surface of considerable local relief is present, the thickness of the older alluvium varles greatly from place to place. Where saturated the older alluvium contains the main aquifers in the area.

The older fan deposits of Pleistocene age (map symbol Qof) consist of weakly consolidated fanglomerate or unsorted unbedded boulder gravel occurring as isolated erosional remnants. The materials are mainly of granitic origin but fragments of basalt, andesite, dacite, and metamorphic rocks are common. The unit is nearly everywhere above the water level in wells and is unsaturated. However, the attitude of this unit suggests that locally it extends beneath the younger or older alluvium in the valley and where saturated may yield small quantities of water to deep wells. 
The younger alluvium of Recent age (map symbol Qyal) is mostly gravel, sand, and silt, and overlies the older units beneath the central parts of the valleys. These deposits are generally above the water table except in the lower parts of the valley, where they may yield small amounts of water to shallow wells.

The younger fan deposits of Recent age (map symbol Qyf) are mostly poorly sorted boulders, arkosic gravel, sand, silt, and clay derived from nearby hills or mountains. The materials have been transported only a short distance and malnly represent mudflow or slope-wash debris. Near the hills and mountains the younger fan deposits are coarse grained, but they become finer with increasing distance from the areas of active erosion. These deposits are poorly sorted and poorly permeable, are usually above the water table, and are believed to be unpromising sources of water.

Playa deposits of Recent age (map symbol Op) occur principally at Koehn Lake, the lowest point in Fremont Valley, and at the base level of some minor drainage areas. They consist principally of silt and clay and minor amounts of sand, are of low permeability, and where saturated usually contain water having a moderate to very high dissolvedsolids content.

Unconsolidated coarse to fine dune sand (map symbol Qds) occurs in the lower parts of the valleys. The dunes are, in part at least, actively drifting; locally some small interdune playas are included in the area shorm as dune sand on plate 1. 
Lakeshore deposits of Recent age (map symbol Qls) occur locally near the old shorelines of large perennial lakes which formerly existed in the lowest parts of the valleys. These deposits consist mainly of coarse gravel and sand but are everywhere above the water table, are not saturated, and therefore do not yield water to wells.

In 1958 the water levels in wells in Fremont Valley ranged from above the land surface in the lowest part of the valley near Koehn Lake to more than 625 feet beneath the higher alluvial slopes north of the Muroc fault and more than 400 feet beneath the alluvial fans extending into the valley from the Rand Mountains east of Koehn Lake.

Recharge to the basin occurs by subsurface ground-water outflow from the Chaffee area (Kunkel and others, 1957) and the North Muroc basin through the older alluvium between Desert and Castle Buttes (pl. 1), from runoff from the directly tributary mountains and hills, and in very minor arnounts by deep penetration of rain during infrequent periods of heavy precipitation.

The ground water in Fremont valley is moderately to highly mineralized. The highest concentration of dissolved solids, about 28,000 ppm (parts per million), occurs in shallow wells near Koehn Lake. The waters of best quality are from wells drilled in the alluvial fans and the higher slopes of the younger alluvium in the southwestern part of the valley, where the dissolved-solids content is only about 400 to $60 \mathrm{cpm}$. 
In North Muroc basin (pl. 1) in 1958 the vater levels in wells were about 80 to 100 feet below the land surface beneath the central part of the basin and more than 250 feet beneath the higher alluvial slopes near the town of Boron. Ground water in the North Muroc basin moves from the surrounding hills or the adjacent ground-water basins (or subbasins) northwestward between Castle and Desert Buttes, through a low topographic divide underlain by alluvium, to Fremont Valley. The ground water in the North Muroc basin has a low to moderate concentration of dissolved solids. The highest concentration of dissolved solids, about 1,000 ppm, occurs in wells near Boron. The waters of best quality come from wells drilled near the western part of the basin, where the dissolved-solids content locally is less than $500 \mathrm{ppm}$.

Three areas are underlain mainly by younger alluvium in the generally uplifted area north of the North Muroc basin and south of the Rand Mountains. Several large northwest-trending faults strike across the area, and bedrock crops out in numerous hills east of Castle Butte. The largest of the three alluvial areas is between the Rand Mountains and the Lockhart fault (pl. 1). The occurrence and movement of ground water in the area is imperfectly known vecause of the lack of wells. However, meager data suggest that subsurface flow from the area enters either the Harper Valley of Kunkel (1956) or the other and smaller subbasins to the south, and thence enters North Muroc basin. 
In the area east of Castle Butte, north of the North Muroc basin and south of the Lockhart fault, there are two valley areas underlain by younger alluvium which contain ground water. Each of these two areas contains several large-capacity irrigation or industrial supply wells.

Recharge to the three valley areas east of Castle Butte occurs mainly from runoff from the local hills and mountains. Discharge occurs by pumping and by subsurface flow to the North Muroc basin and eventually to Fremont Valley.

The quality of water is suitable for most domestic, irrigation, and industrial use.

Of the approximately 700 square miles of the Fremont Valley area, more than half is underlain by consolidated rocks where wells have not been drilled. The valley areas underlain by unconsolidated deposits contain 370 wells which are shown on plate 1 and are described in table 1.

Table 2 lists cross indexes of Geological Survey well numbers and the numbers previously given to the same wells by other workers in the area.

Table 3 lists all available water-level measurements, and table 4 lists all drillers' logs of wells.

Table 5 contains chemical analyses of water from wells. The analyses were made by the agencies shown in the table. 
ECONOMIC ASPECTS OF GROUND WATER IN THE AREA

The principal towns in the Fremont Valley area are Randsburg near the northeast margin and Boron near the southeast corner. Boron was named for the borate minerals mined near the town by the U. S. Borax and Chemical Corp. The borate deposit is the largest ever discovered in the United States.

On the basis of economic development, the Fremont Valley area can be divided roughly into three parts. The economy of the southern part of the area, extending from Boron and U. S. Highway 466 to Castle Butte (pl. 1), is based almost entirely on the mining of the borate deposits near Boron, the residential development resulting from the employment of workers at nearby Edwards Air Force Base south of U. S. Highway 466, and commerce with the travelers using the highway. In Fremont Valley, which extends about from Castle Butte to several miles northeast of Koehn Lake, the economy is based almost wholly on irrigated agriculture. In 1958 roughly 8,000 acres of land was irrigated by pumping ground water at three large and several smaller ranches.

Finally, in the area near Randsburg the economy formerly was based on the mining of gold, silver, tungsten, and other metals and minerals. In recent years, however, many of the mines have closed and only limited mining is now done. In many instances the difficulty of obtaining water is great, and long pipelines have been bullt from the lower parts of the valley where it is possible to drill largecapacity wells to supply water to the mines and mills. 
The well-numbering system used in the Fremont Valley area conforms to that used in virtually all ground-water investlgations made by the Geological Survey in California since 1940. It has been adopted as official by the California Department of Water Resources and by the California Water Pollution Control Board for use throughout the State. Wells are assigned numbers according to their location in the rectangular system for the subdivision of public land. For example, in the number $11 / 8-33 L 1$, assigned to a well show on plate 1 , the part of the number preceding the bar indicates the township ( $T$. $11 \mathrm{~N}$. ), the part between the bar and the hyphen indicates the range ( $R .8$ W.), the number between the hyphen and the letter indicates the section (sec. 33), and the letter indicates the 40-acre subdivision of the section as shown in the accompanying dlagram.

\begin{tabular}{|c|c|c|c|}
\hline$D$ & $C$ & $B$ & $A$ \\
\hline$E$ & $F$ & $G$ & $H$ \\
\hline$M$ & $L$ & $K$ & $J$ \\
\hline$N$ & $P$ & $Q$ & $R$ \\
\hline
\end{tabular}

Within the 40-acre tract the wells are numbered serially as indicated by the final digit. Thus, well $11 / 8-33 \mathrm{Ll}$ is the first well to be listed in the $N E \frac{1}{4} S W \frac{1}{1} \sec .33$ (San Bernardino base and meridian). 
Similarly, well 32/37-16RI is in the $\mathrm{SE}_{\frac{1}{4}} \mathrm{SE}_{1}^{\frac{1}{3}}$ sec. 16, T. $32 \mathrm{~S}$, R. 37 E., Mt. Diablo base and meridian. Because all of the wells in the Fremont Valley area are elther in the northwest quadrant of the San Bernardino base and meridian lines or in the southeast quadrant of the Mt. Diablo base and merldian lines, the foregoing abbreviations of the township and range are sufficient.

For well numbers where a dash has been substituted for the letter designating the 40-acre tract, the dash indicates that the well is plotted from unverified location descriptions; the indicated sites of such wells were visited but no evidence of a well could be found. For some wells the letter following the section number is designated $\mathrm{X}$. This indicates that the well has been field located and is accurately plotted with respect to its position on the map, but that the control for the public-land net is too poor to warrant assigning a more accurate location number. 
Dibblee, T. W., Jr., 1952, Geology of the Saltdale quadrangle, Calif.: Cal1fornia Div. Mines Bull. 160, 66 p. 1958, Geologic map of the Castle Butte quadrangle, Kern County, Calif.: U. S. Geol. Survey Mineral Inv. Map MF-170.

Kunkel, Fred, 1956, Data on water wells in Cuddeback, Superior, and Harper Valleys, San Bernardino County, Calif.: U. S. Geol. Survey open-file rept., 73 p.

Kunkel, Fred, and others, 1957, Data on water wells in the Willow Springs, Gloster, and Chaffee areas, Kern County, Calif.: U. S. Geol. Survey open-file rept., $67 \mathrm{p}$.

Stone, R. S., 1957, Ground-water reconnaissance in the western part of the Mojave Desert, Cal1f., with particular respect to the boron content of well water: U. S. Geol. Survey open-file rept., $102 \mathrm{p}$

Thompson, D. G., 1929, The Mohave Desert region, California: U. S. Geol. Survey Water-Supply Paper 578, 759 p. Williams, Cyril, Jr., consultirg engineer, San Francisco, Calif., 1930, Supply investigation in the vicinity of Mojave, Calif.: Prepared for Pacific Portland Cement Co. 


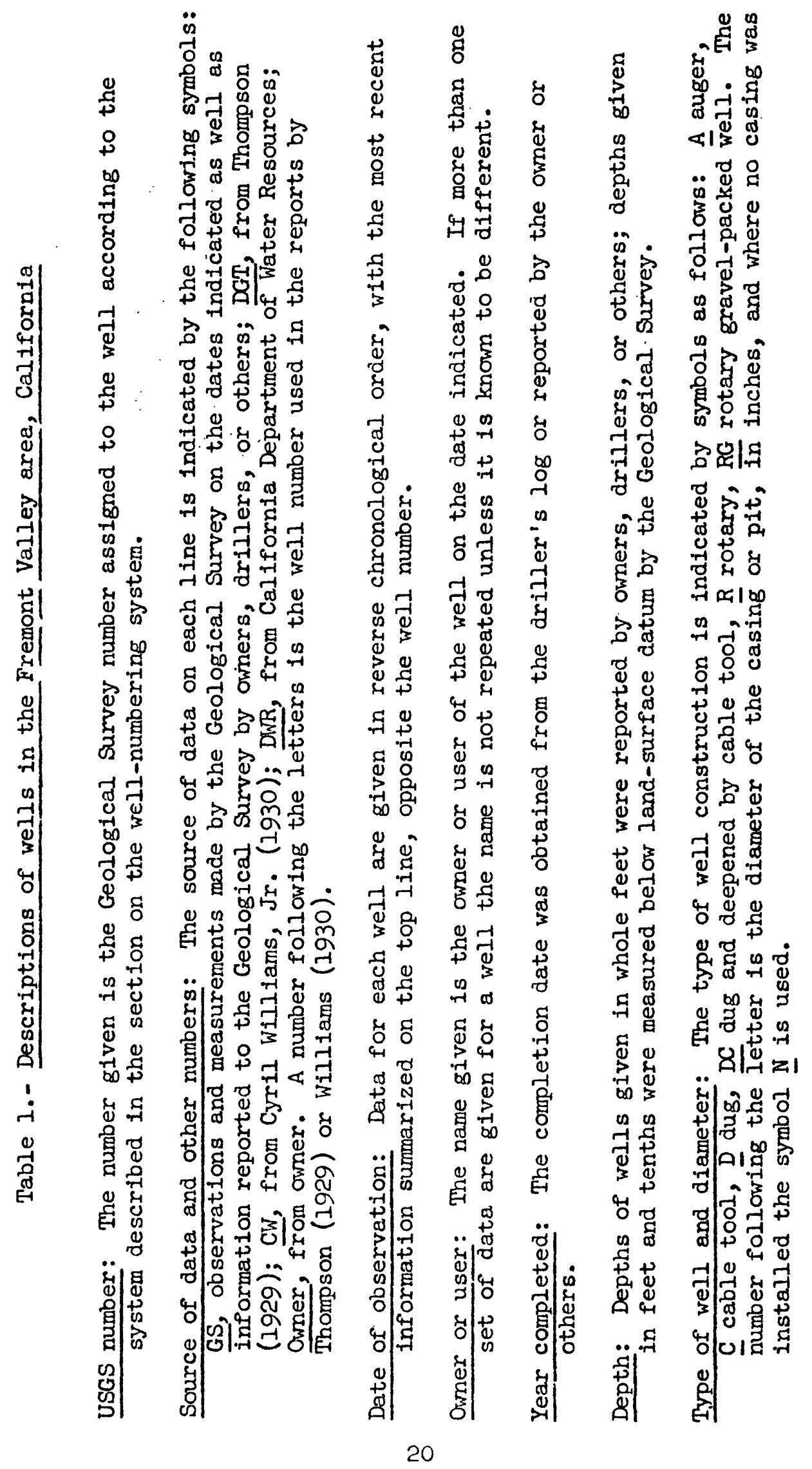




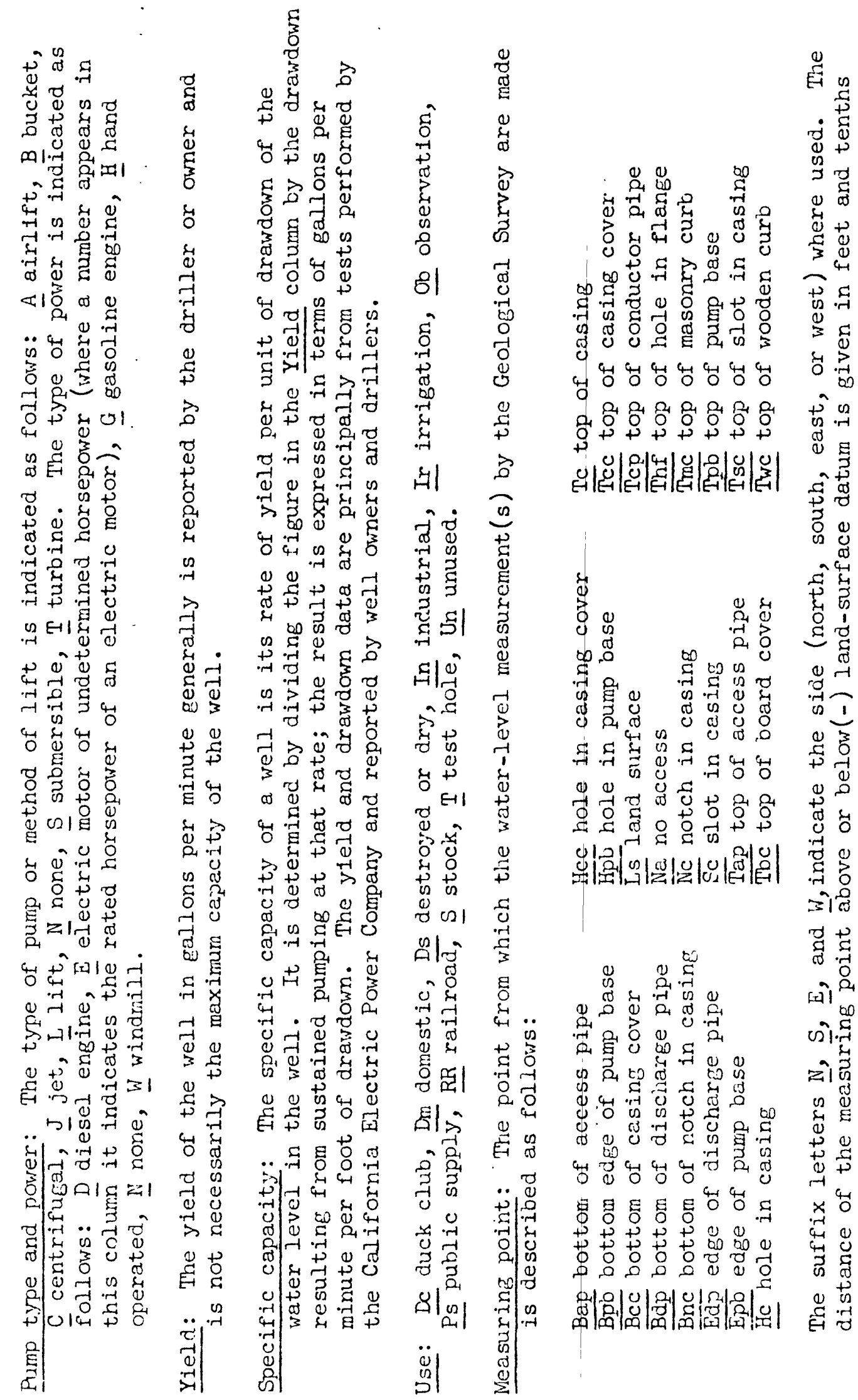




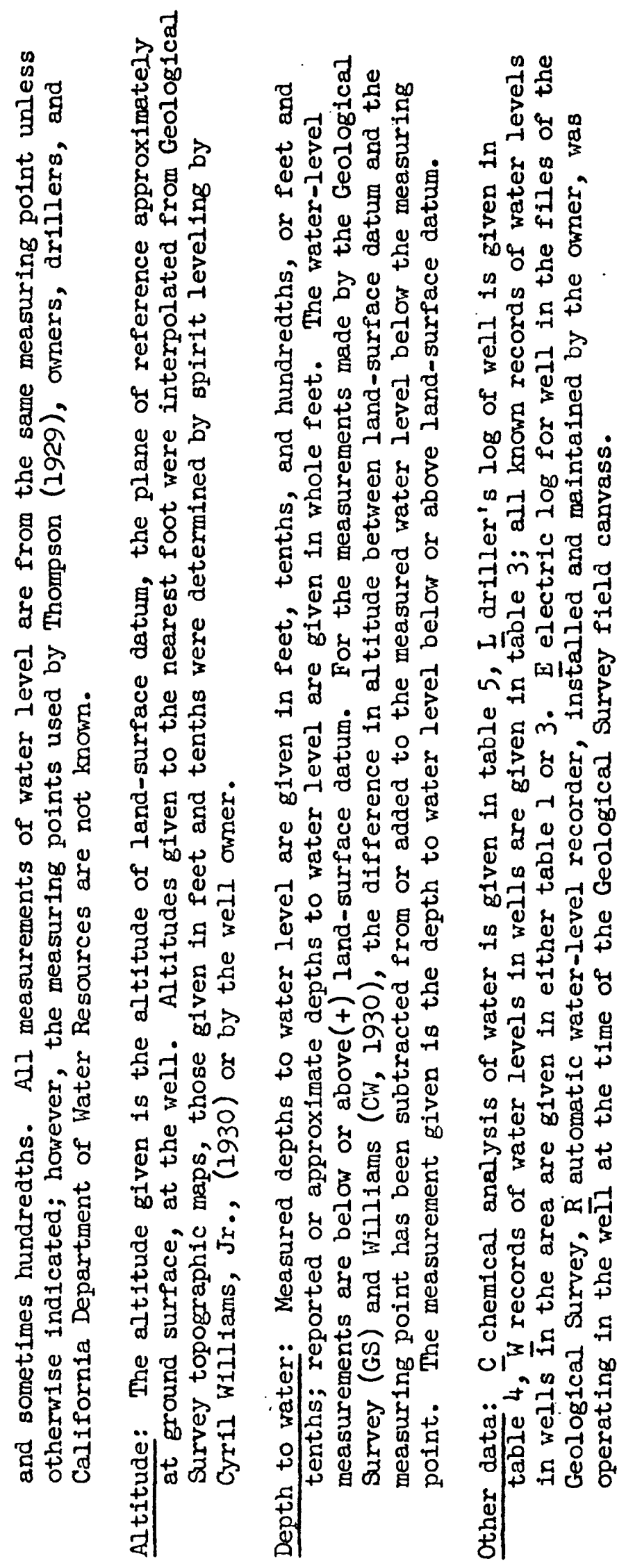




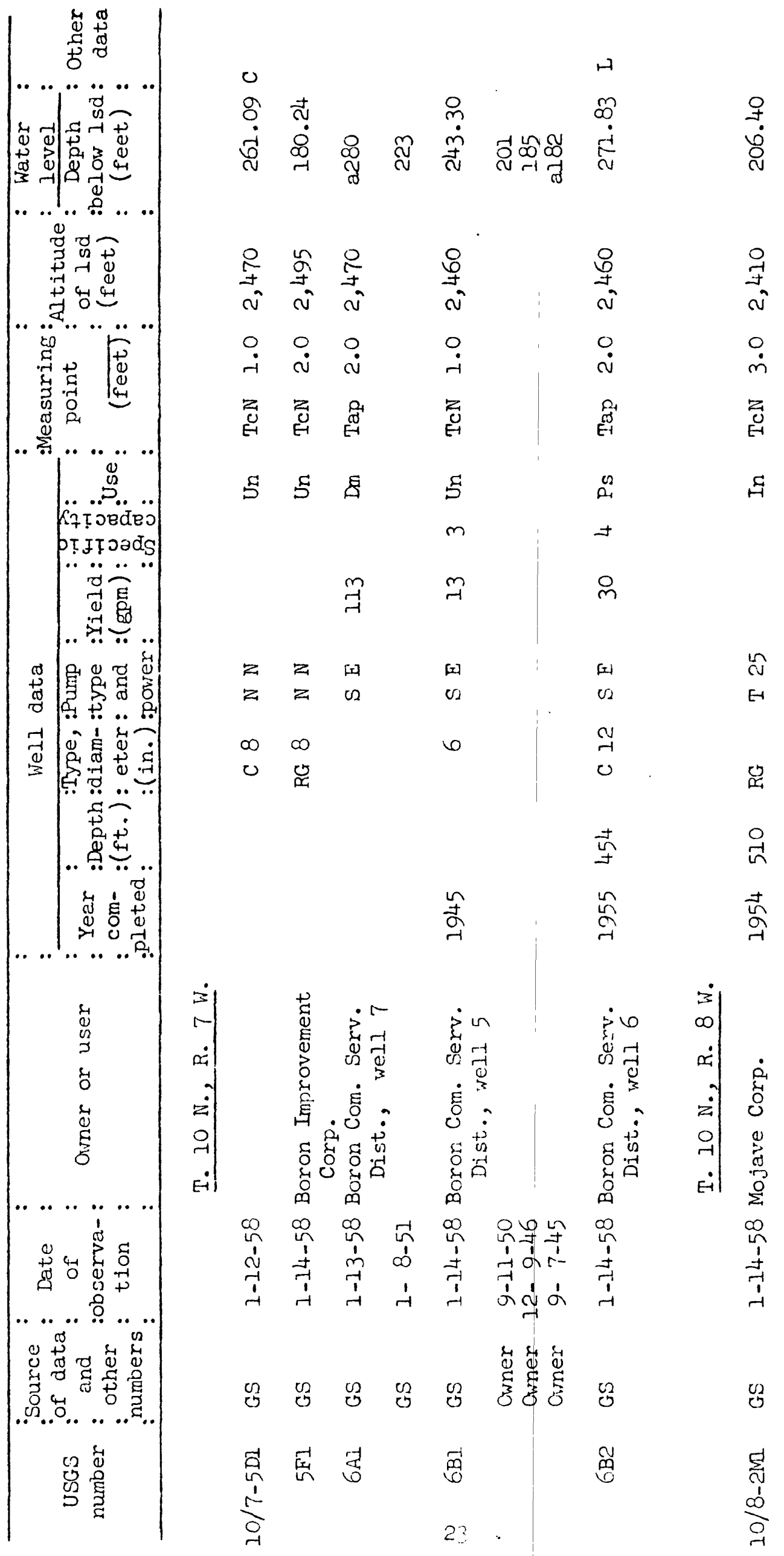




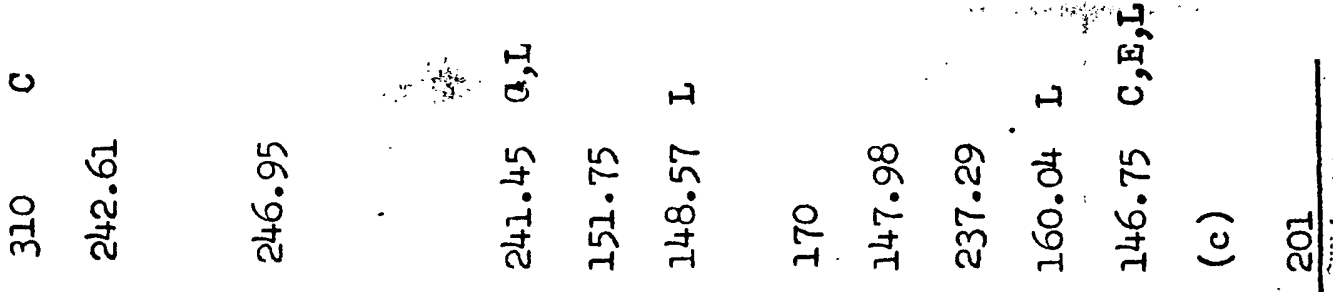

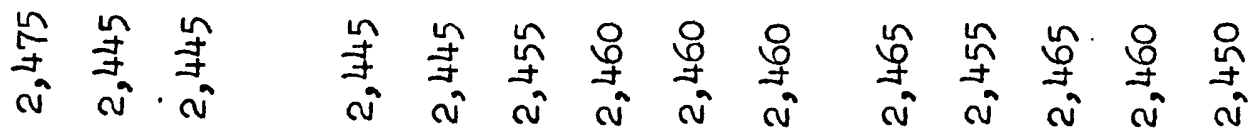

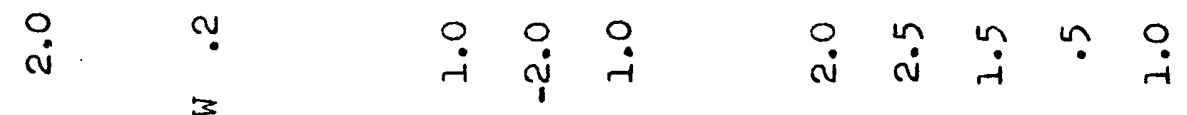

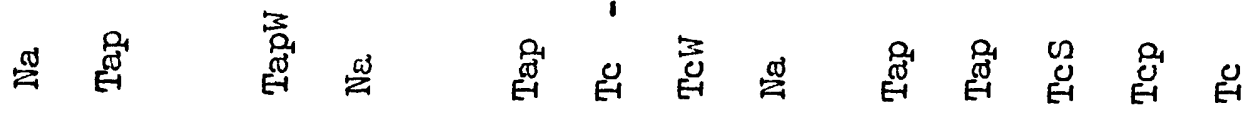

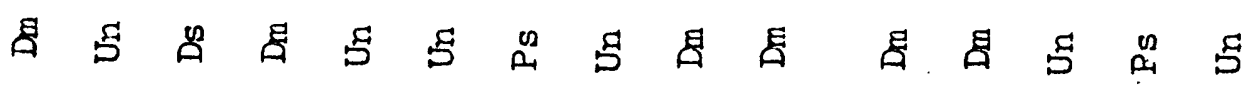
$\dot{m}$.

$+\stackrel{\stackrel{m}{m}}{+}$

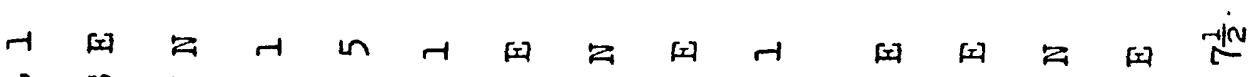

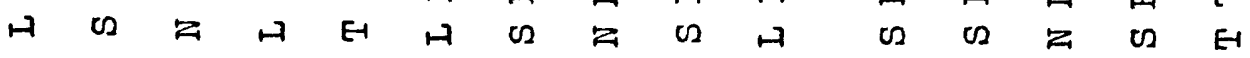

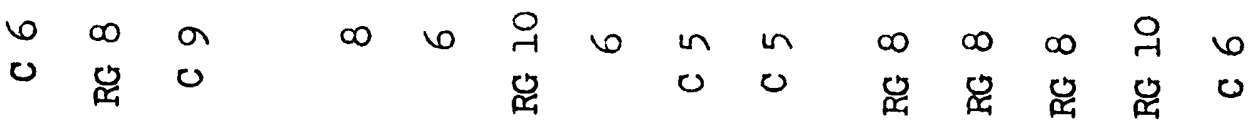

莶

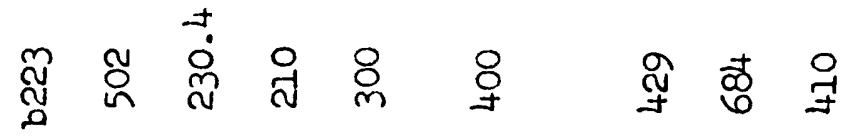

瓷萼号

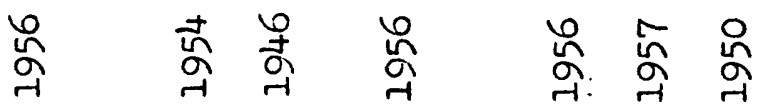

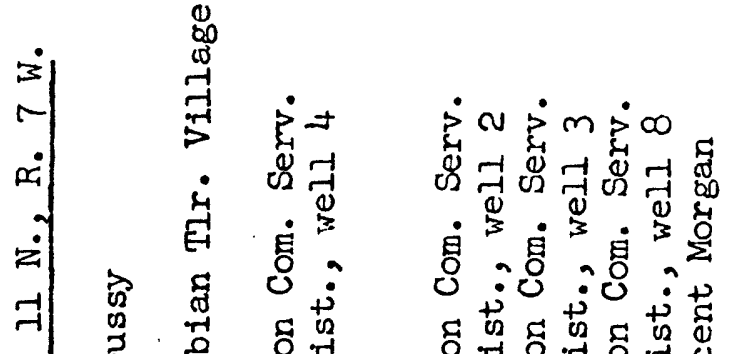

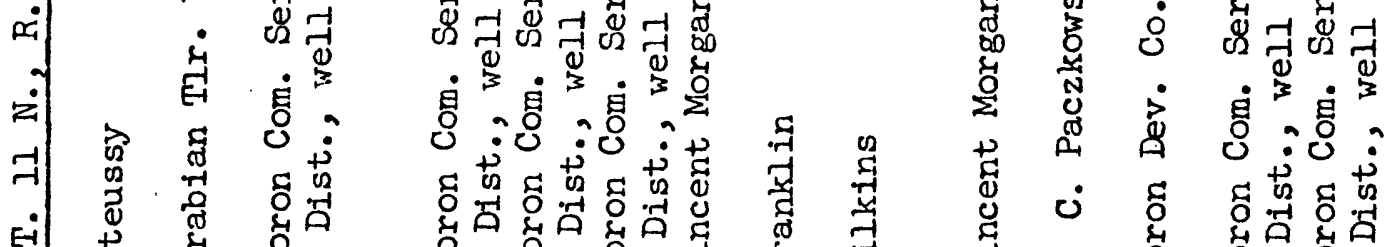

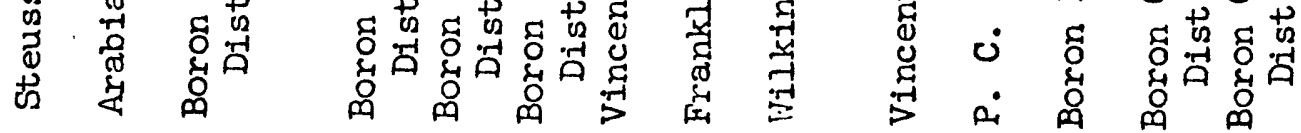

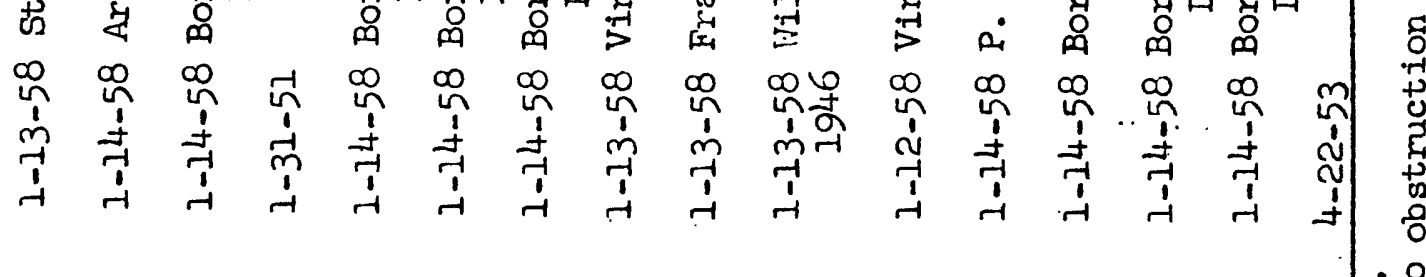
У 兽 


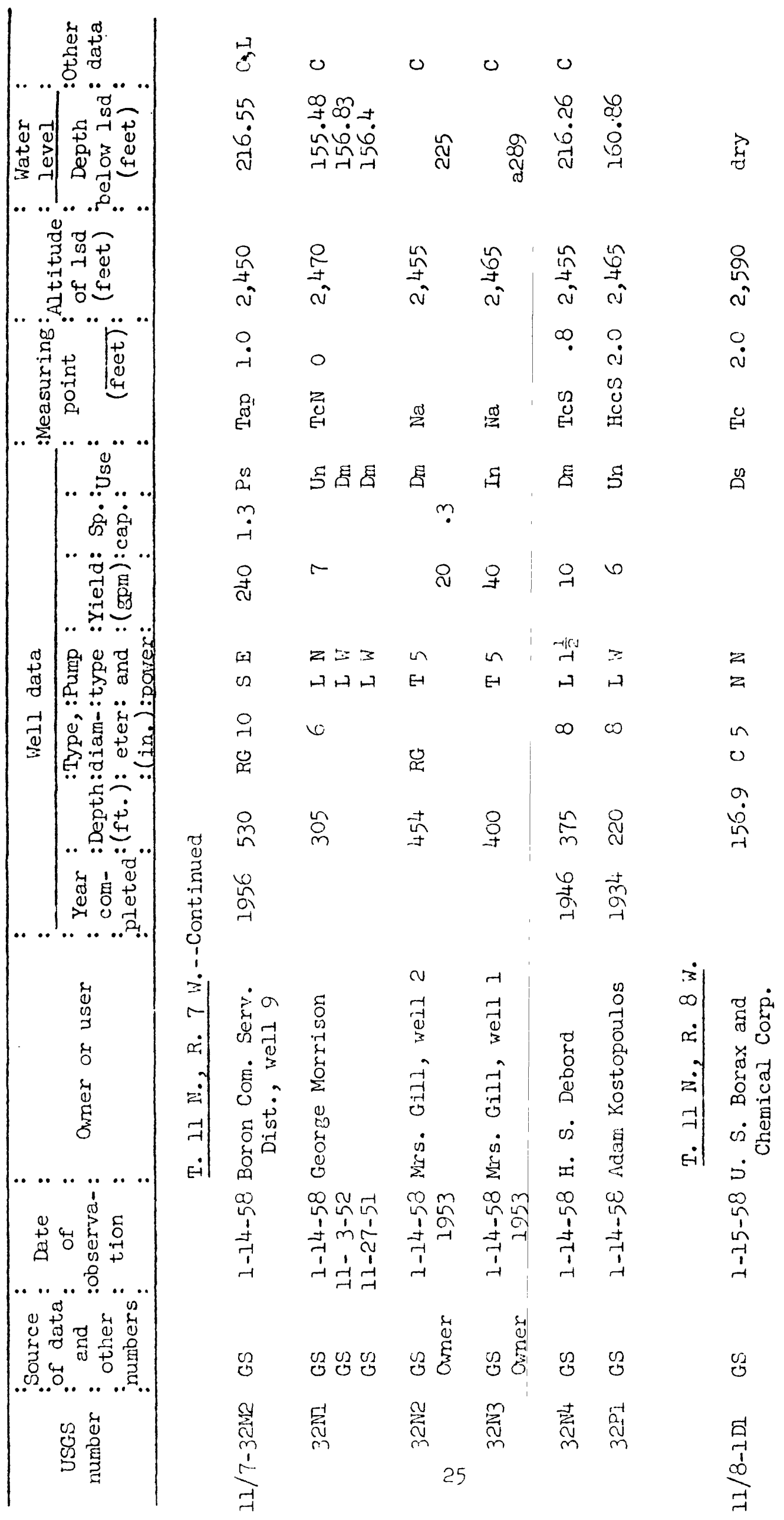




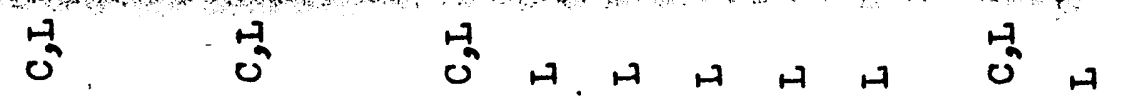

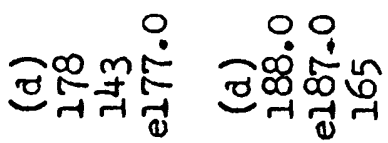

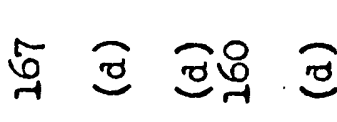

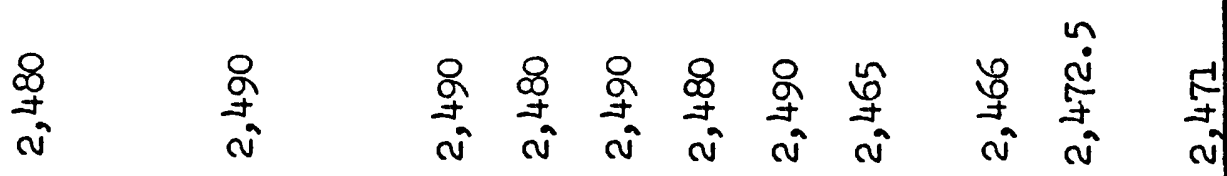

$\stackrel{i}{i} \dot{i}$

总总

离

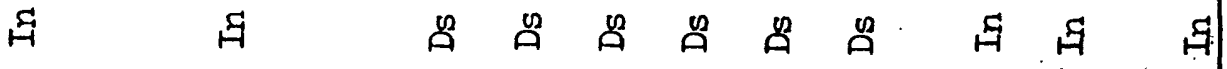

$\stackrel{\infty}{i}$

$\stackrel{4}{4}+4 \stackrel{8}{0}$

$\stackrel{\text { ฯ }}{n}$

in 88

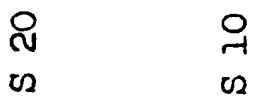

$z z z z z z \quad$ 电

न

z Z Z Z Z Z Z C2 is

4

o

\%

$z= \pm$

足尊

$\underset{m}{P}$

0

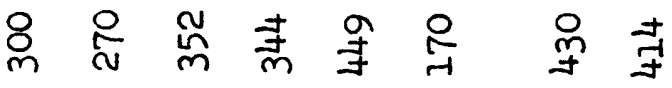

毒

靑

号

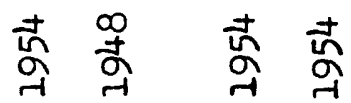

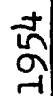

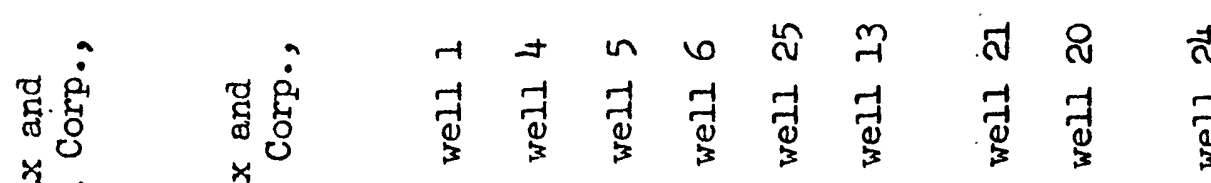
兽哥 vi vi $\dot{0}$ $\dot{0}$

$\dot{b} \dot{\Delta} \dot{s} \dot{s} \dot{s}$

$\dot{5} \dot{0}$

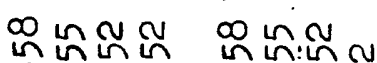

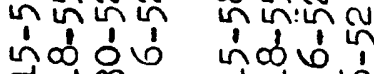
İ

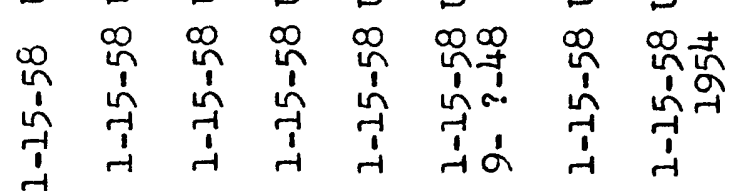

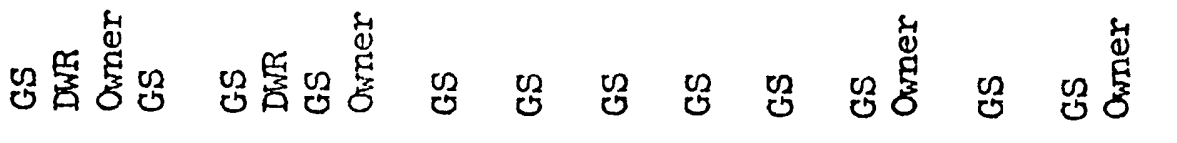
点 苂 $\vec{i}$

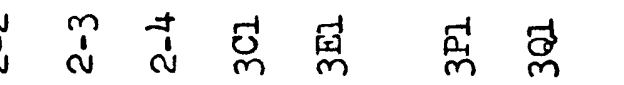




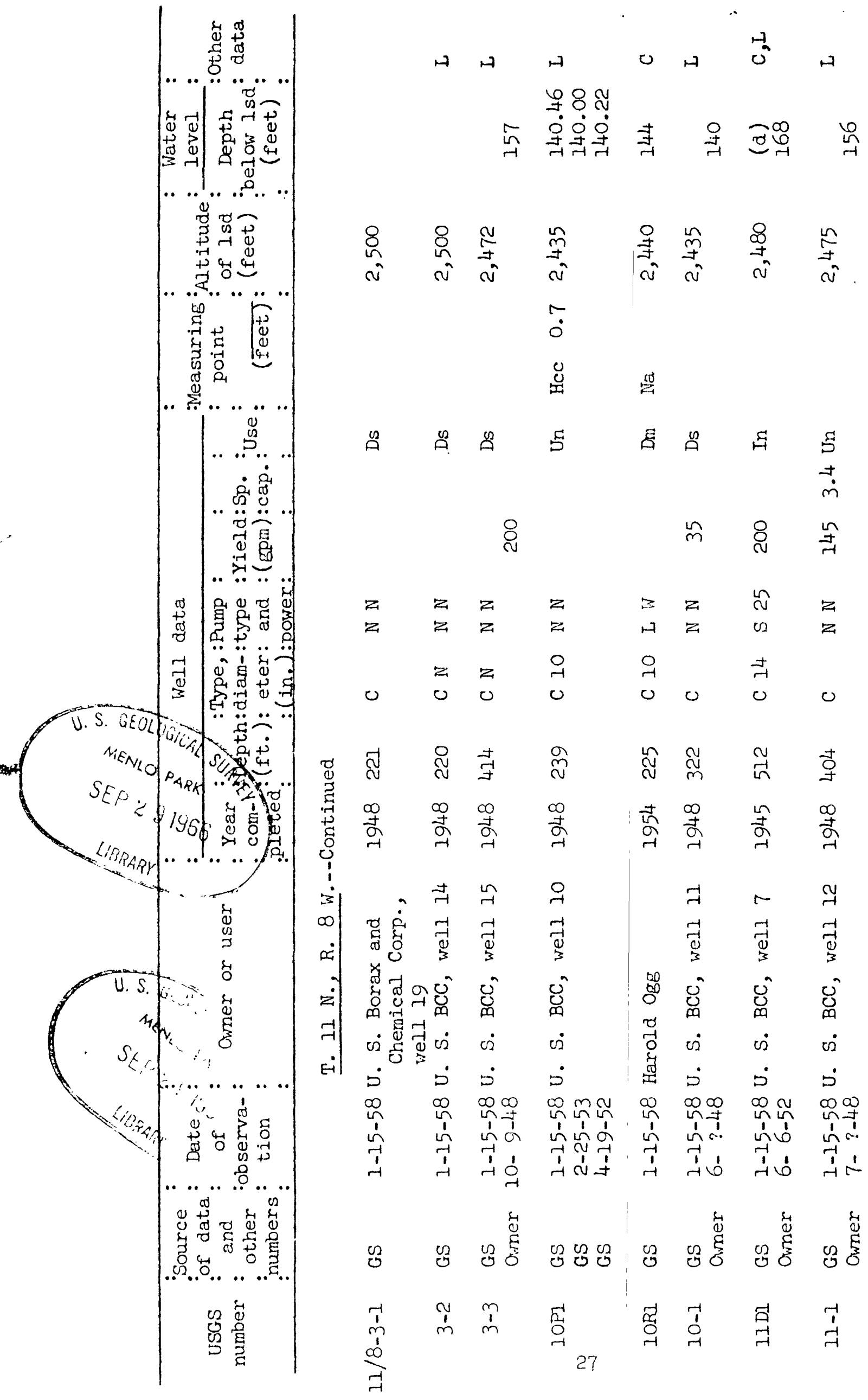




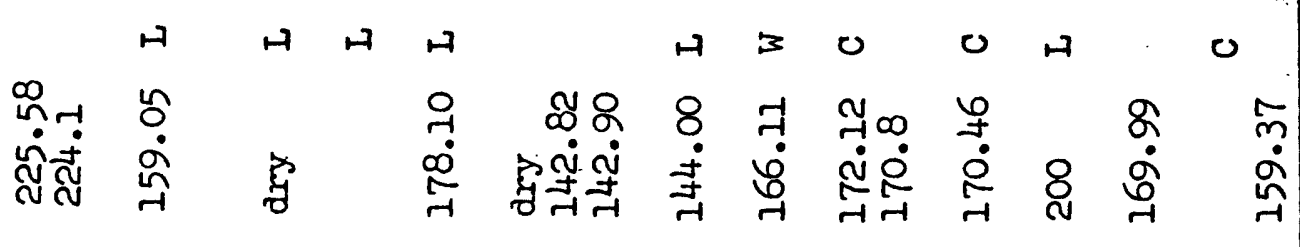

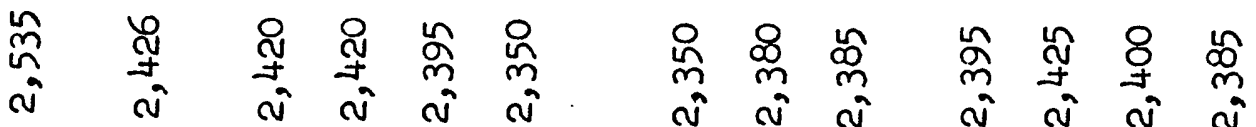

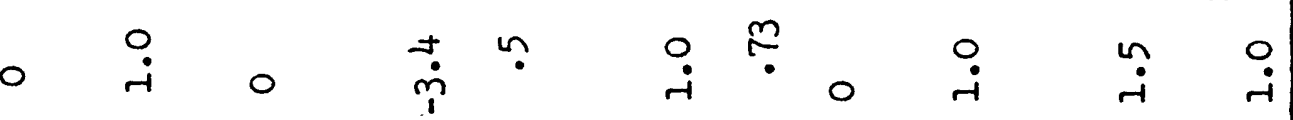

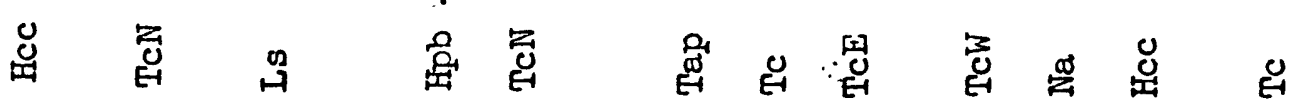

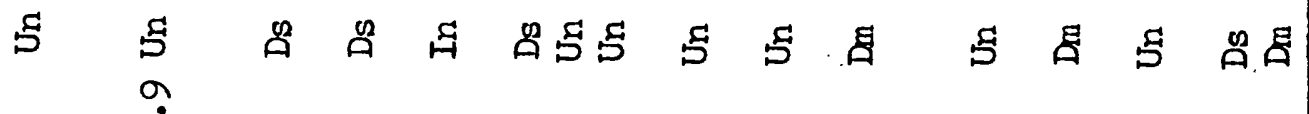

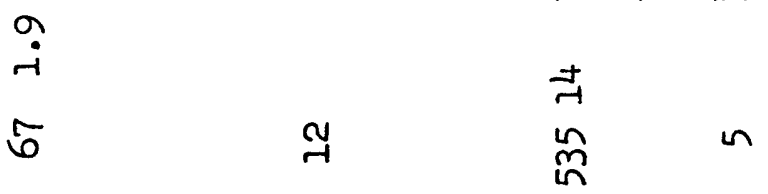

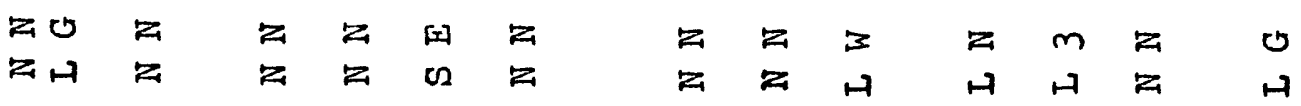

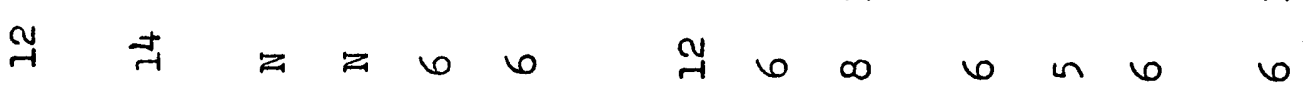

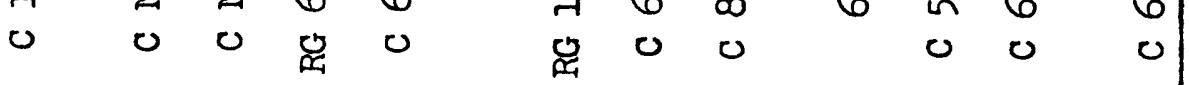

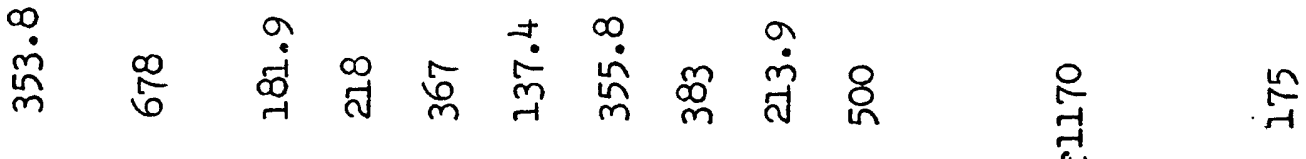

高

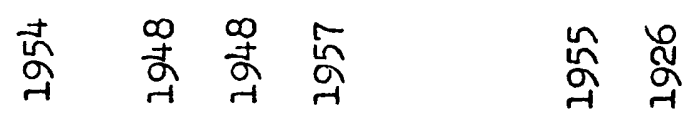

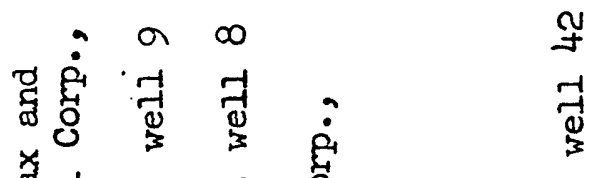

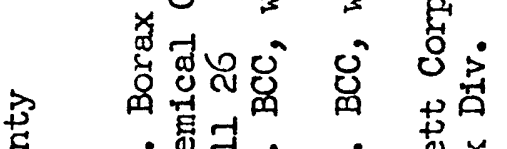

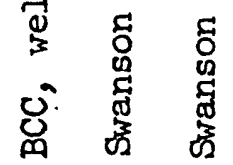

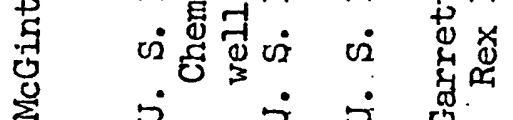

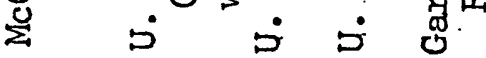

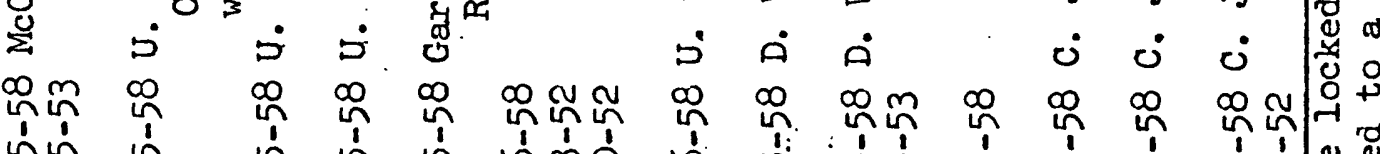
जी

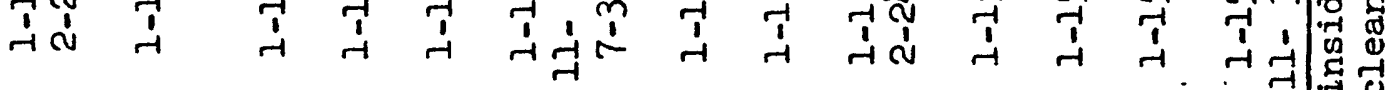

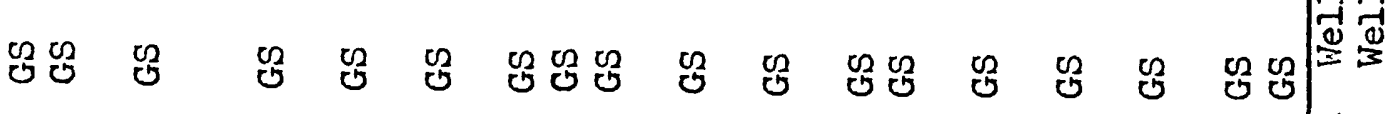

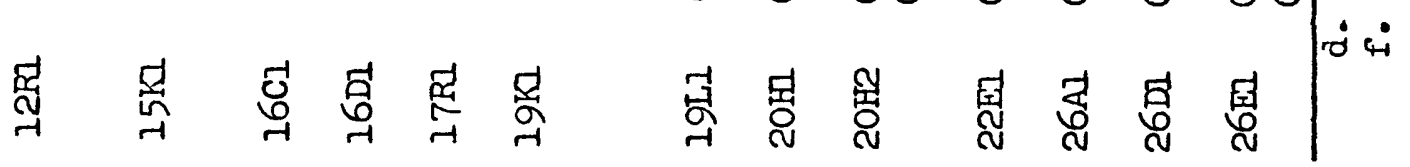




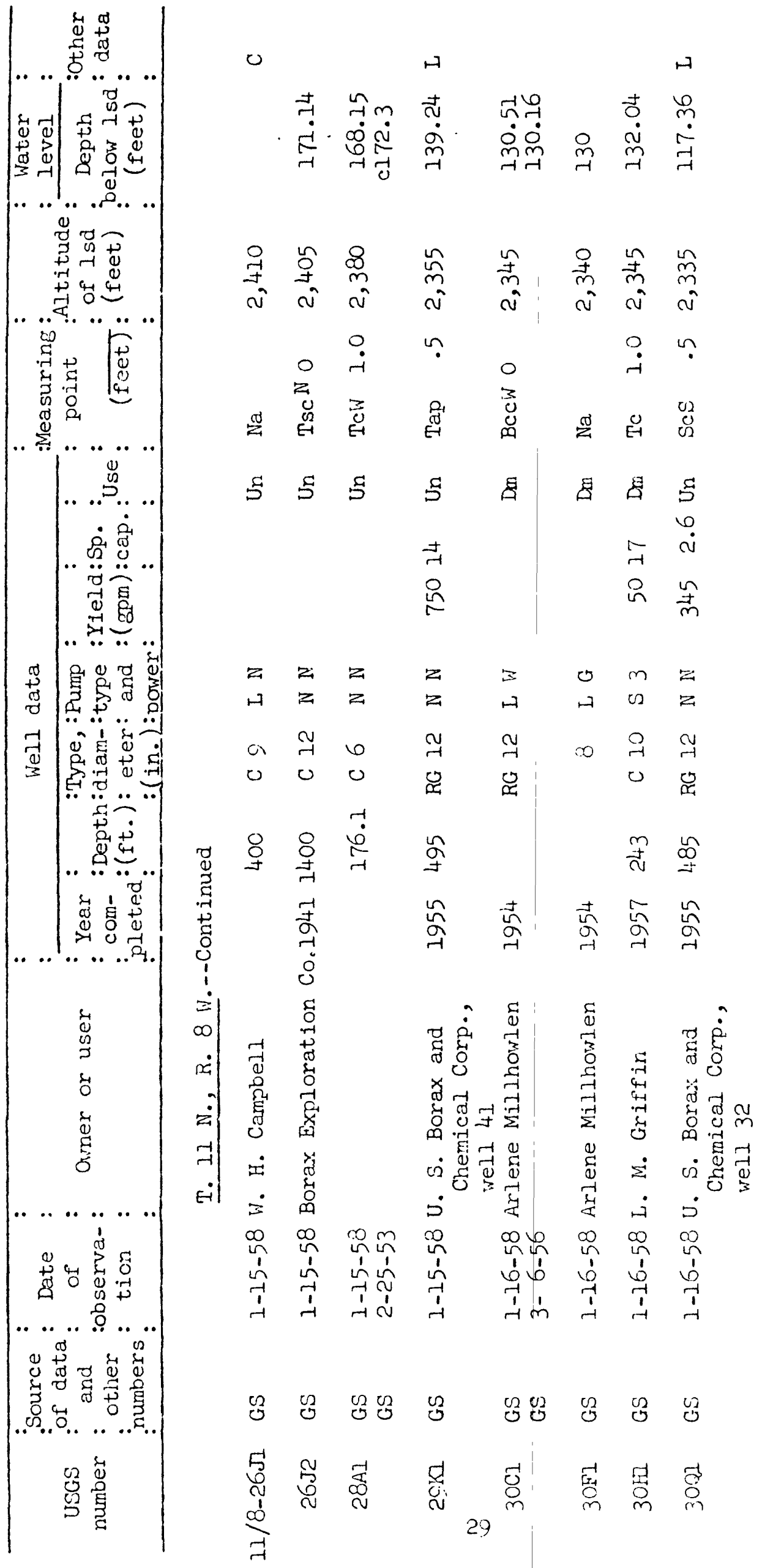




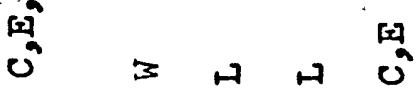

야 \&

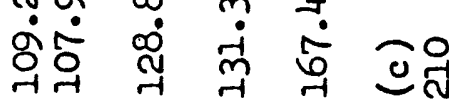

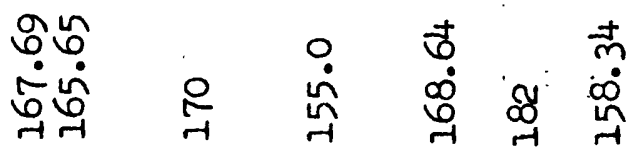

लै

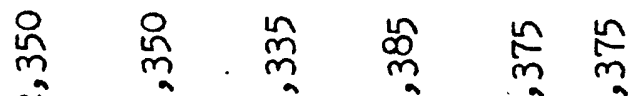

iे रे रे के रे रे

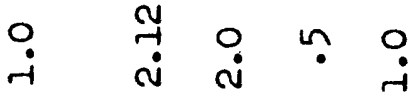

$0 \quad \stackrel{0}{i} \quad$ i

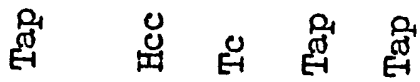

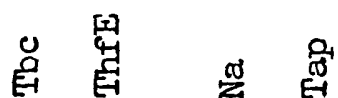

ze

5 5 5 Б 5 م

5 ค ค

?

E

$) \dot{m}$

న

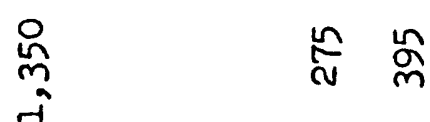

$z \quad z$ 田质的

$\begin{array}{llll}\text { Z } & \text { Z } & 0 & 0\end{array}$

각 가 त

吕 000 诘

ก

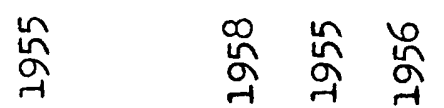

वृ

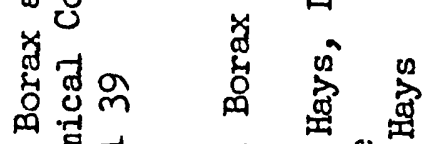

v过势 离

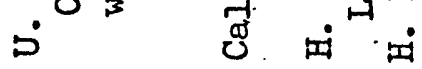

舟舟 $\infty$

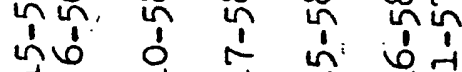

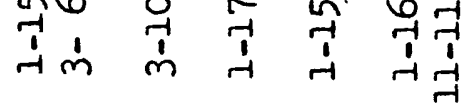

ail

\begin{tabular}{lllll}
$y$ & $z$ & 0 & $\infty$ & \multirow{r}{1}{} \\
0 & $\infty$ & & 0 & 0 \\
0 & 0
\end{tabular}

$\begin{array}{lllll}z & z & 0 & 0 & z \\ z & z & n & n & z\end{array}$

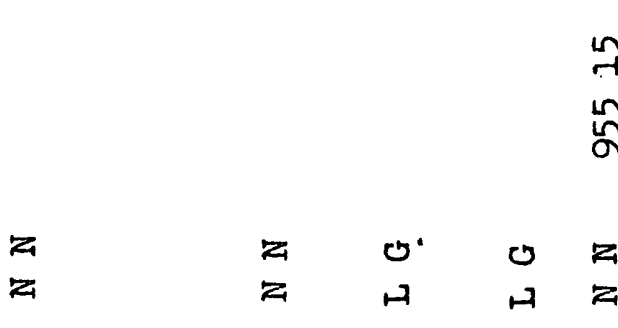

8

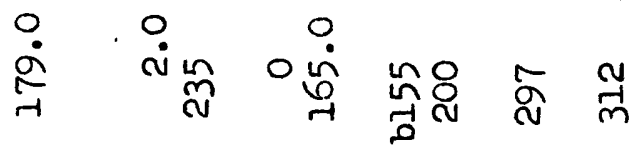

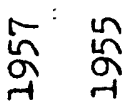

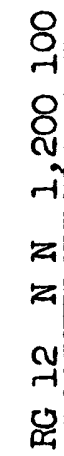

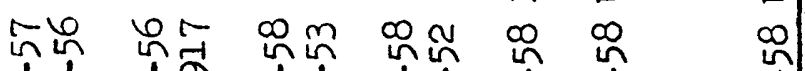

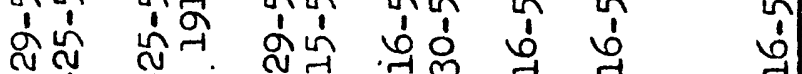

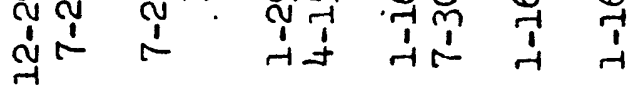

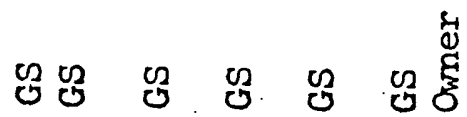

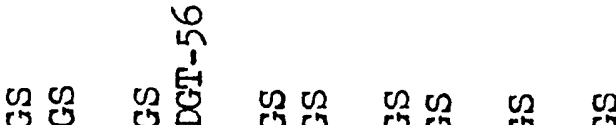

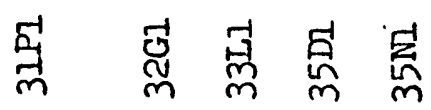

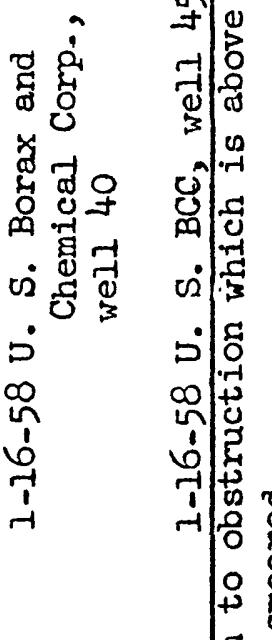

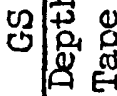

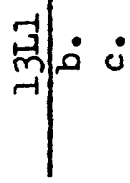




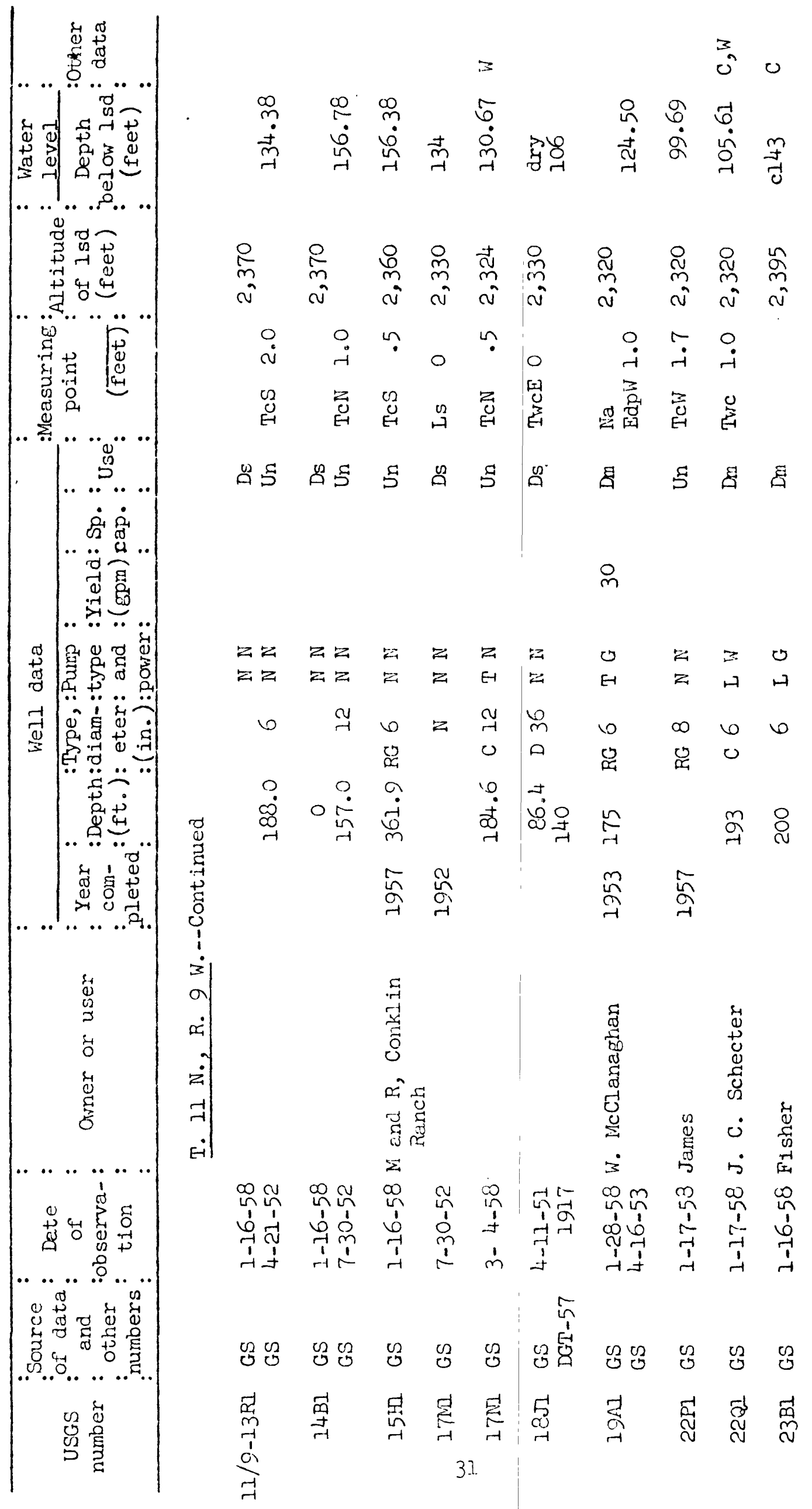




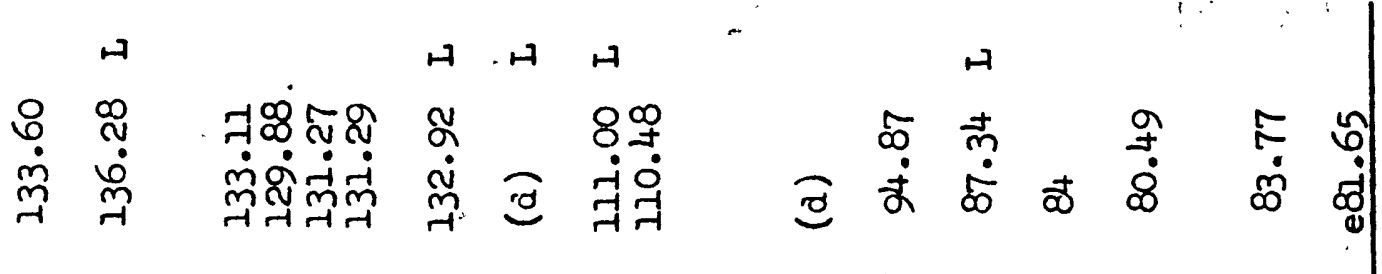

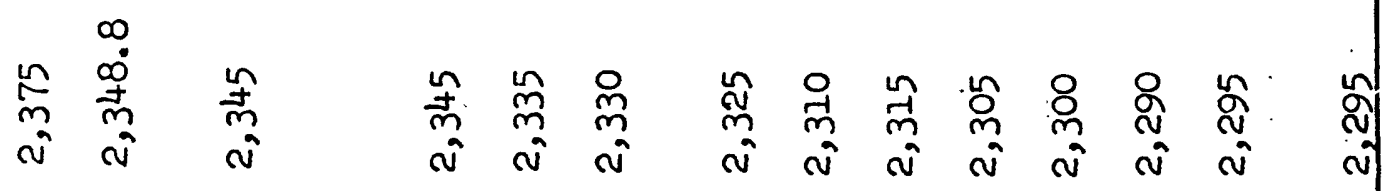

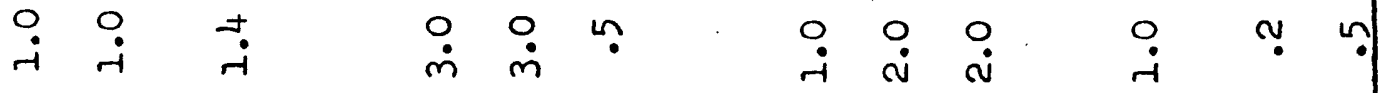

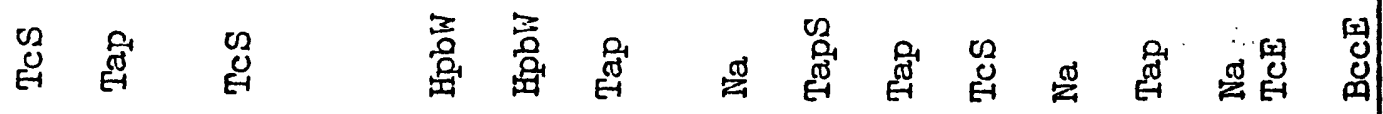

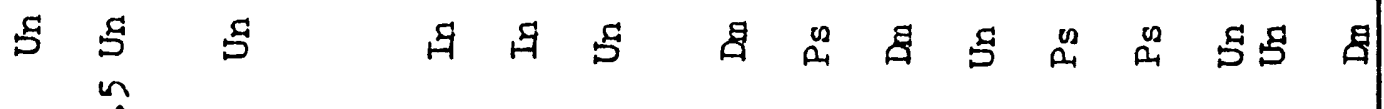

需

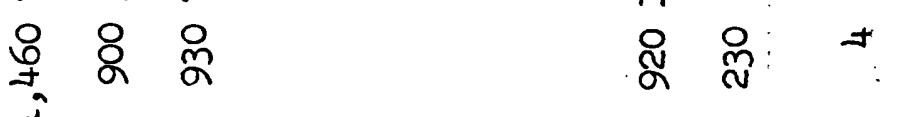

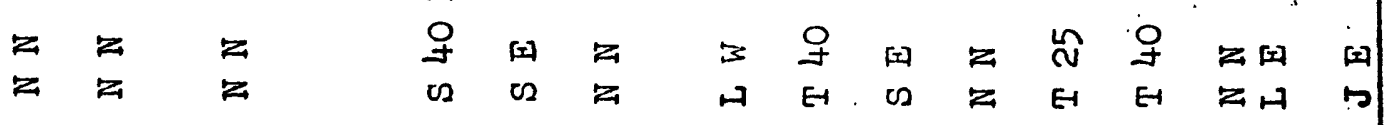

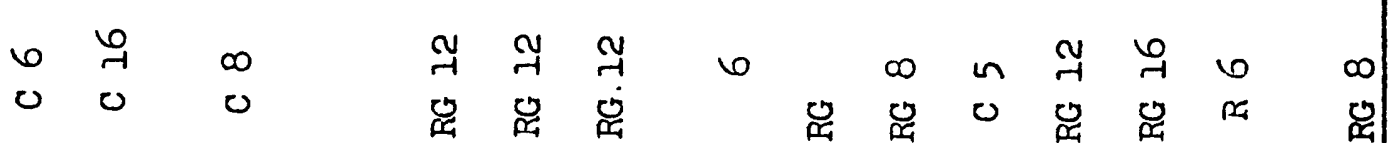

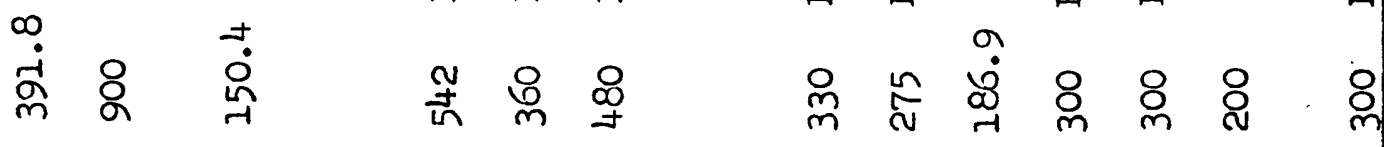

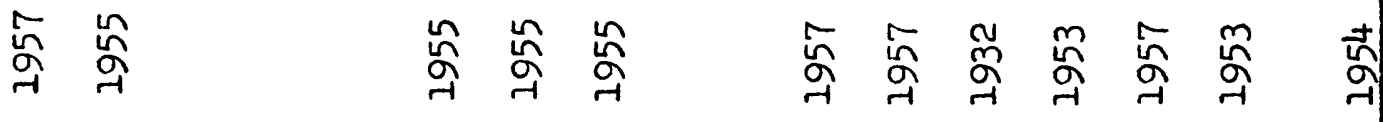

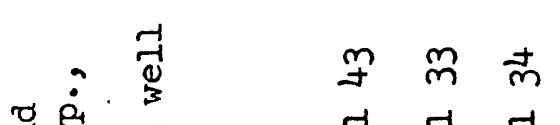

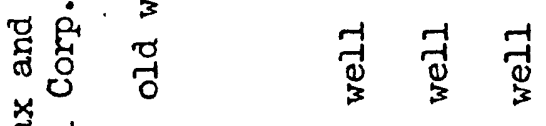

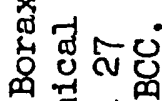

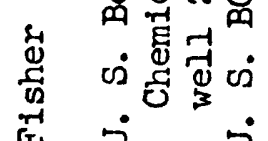

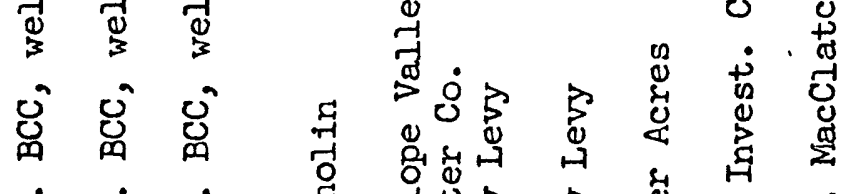

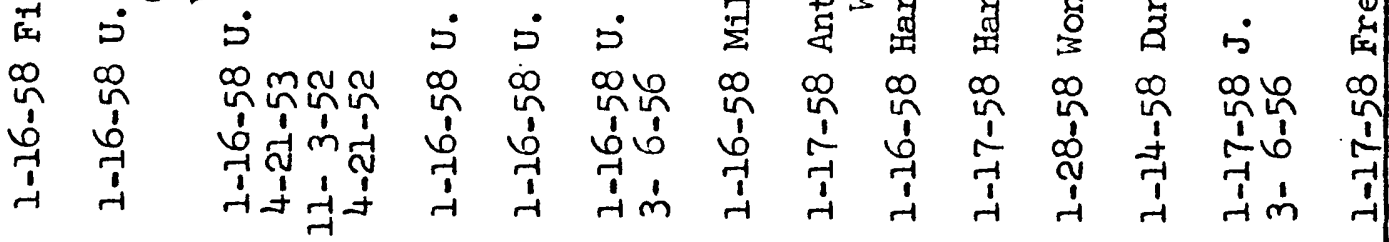

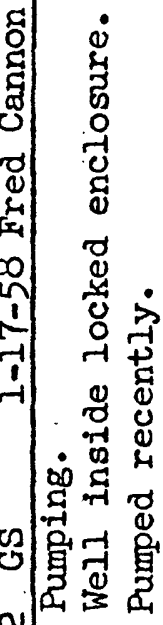

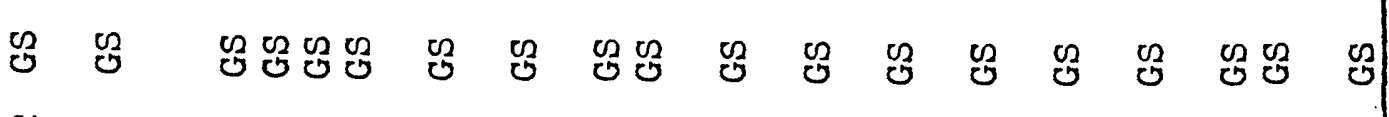

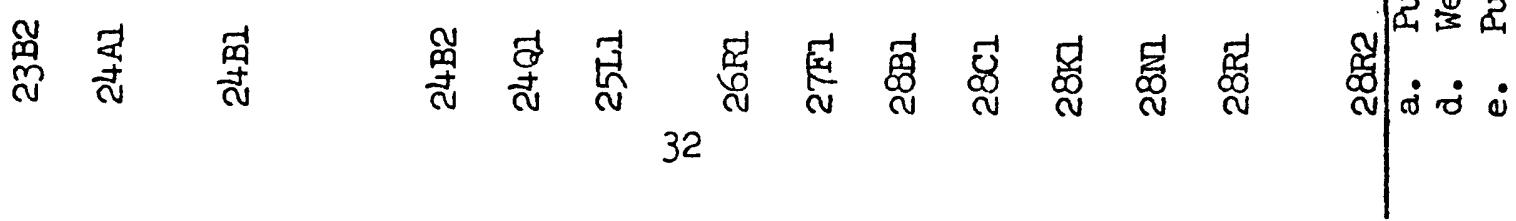




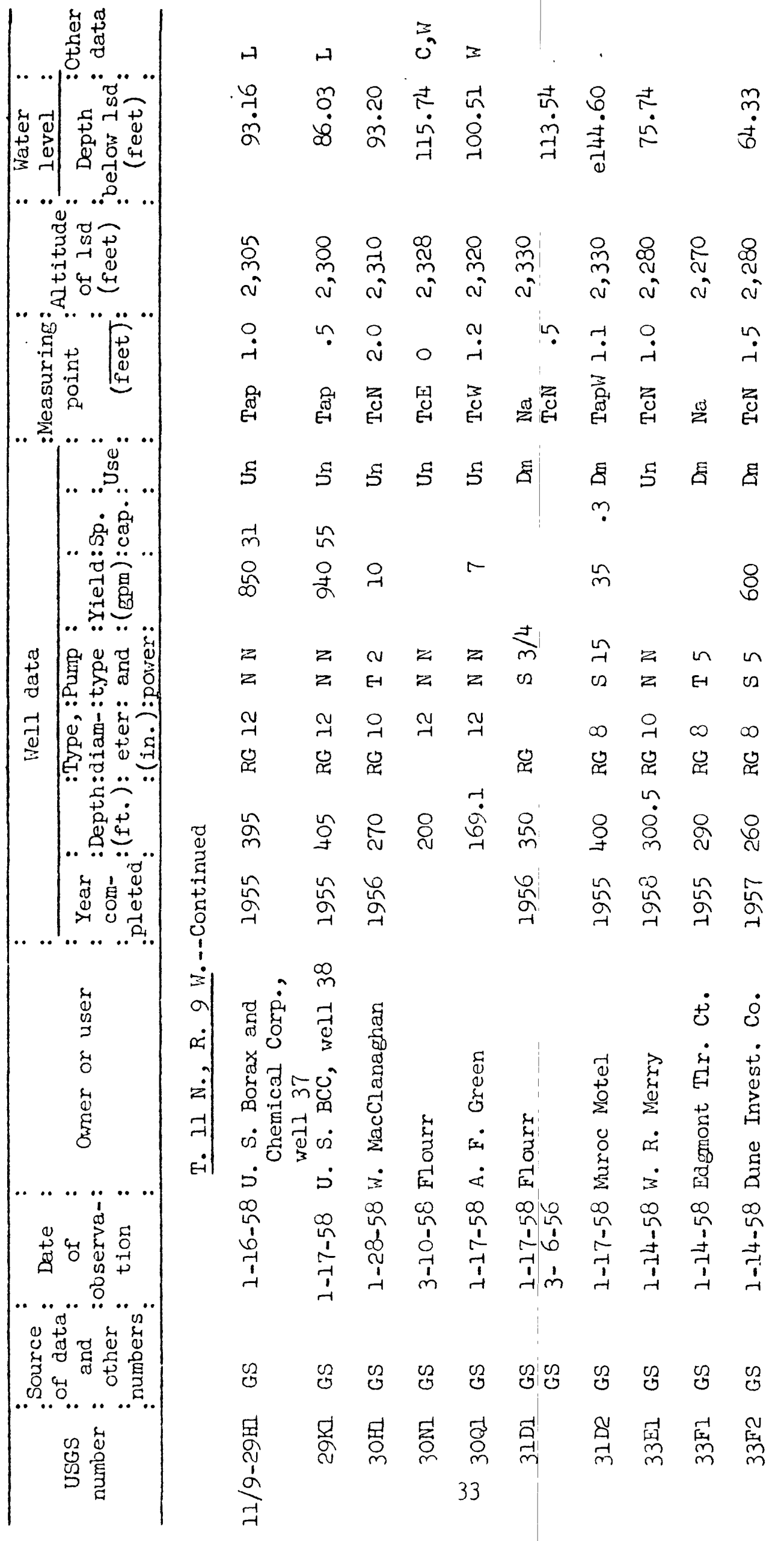




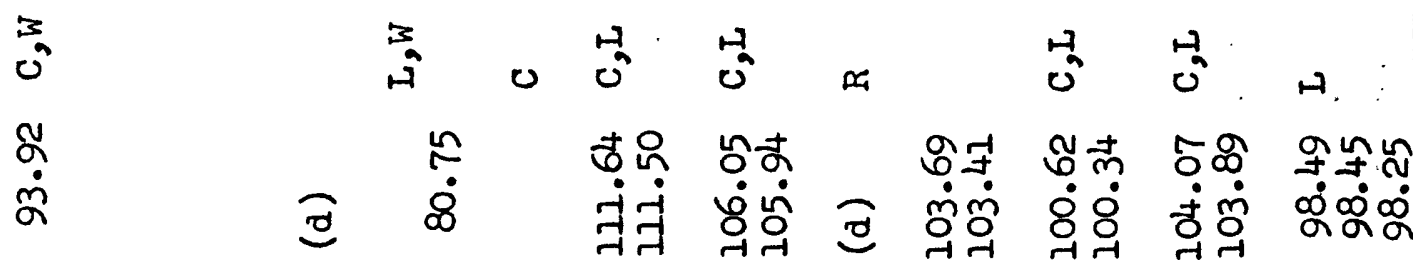

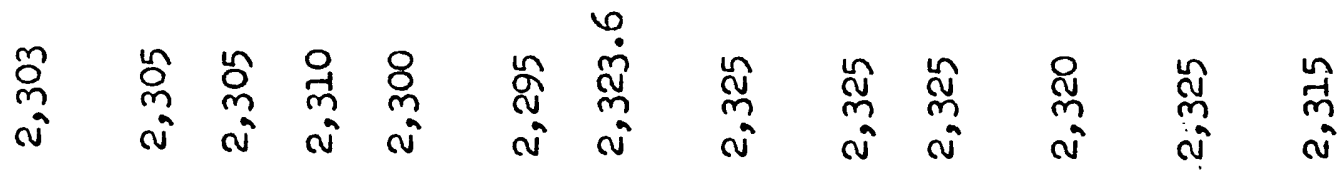

$\because \quad \stackrel{i}{i}$

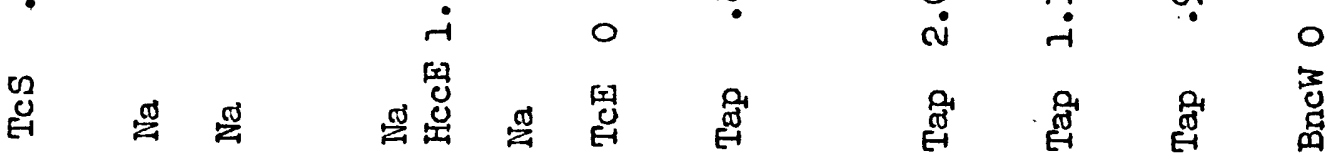

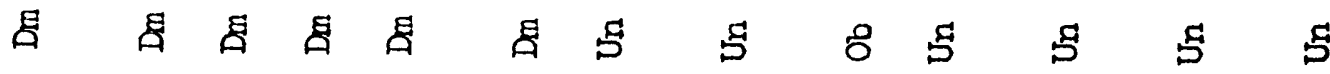

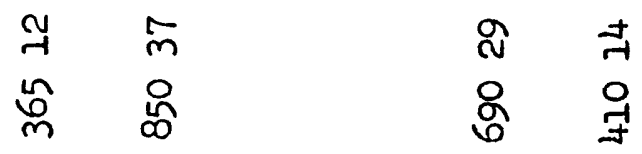

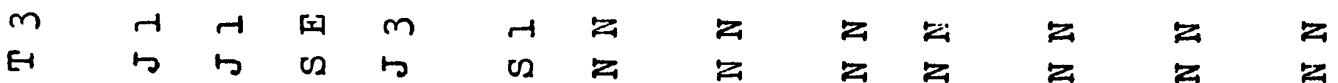

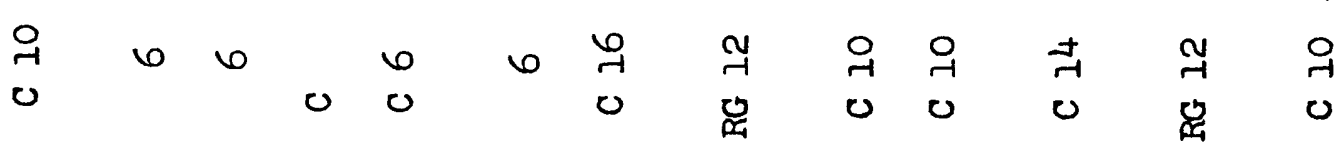

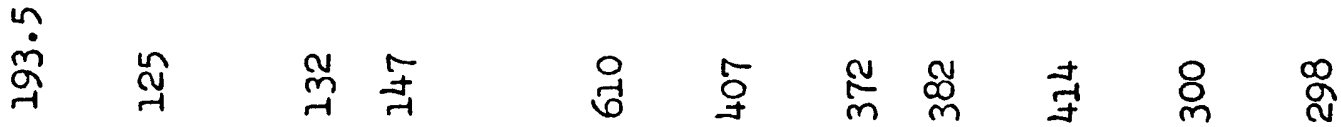

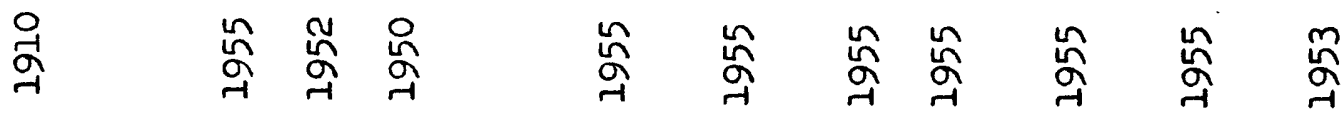

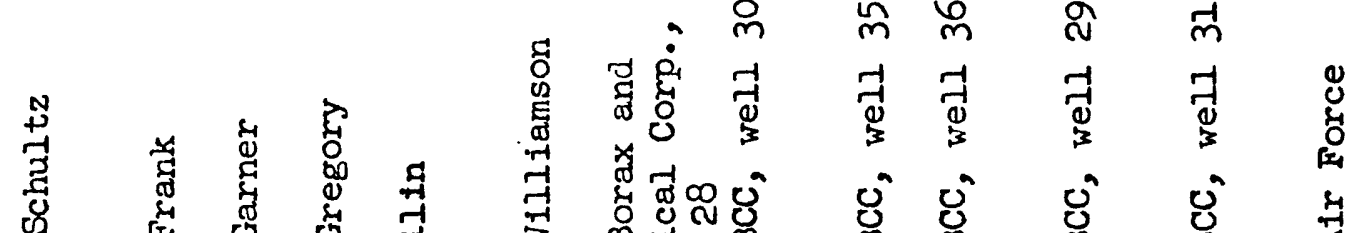

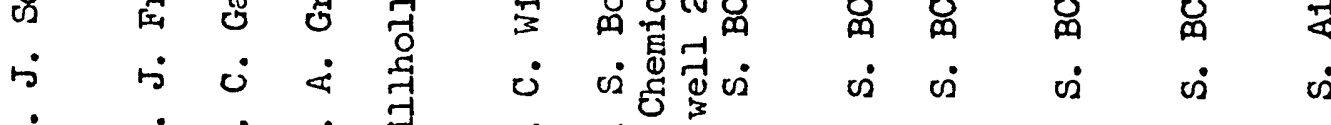

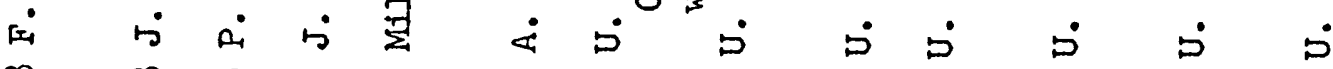

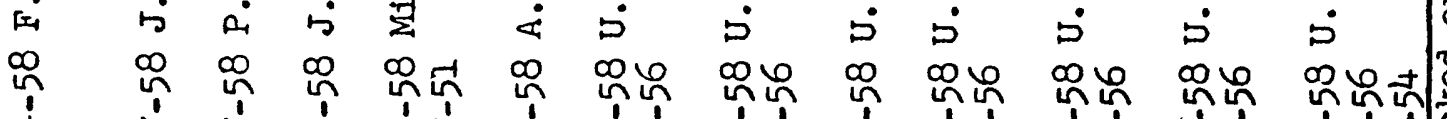

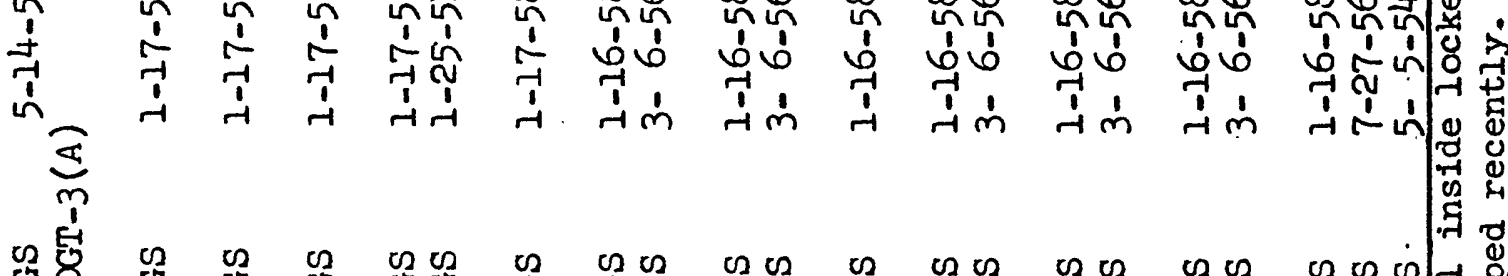

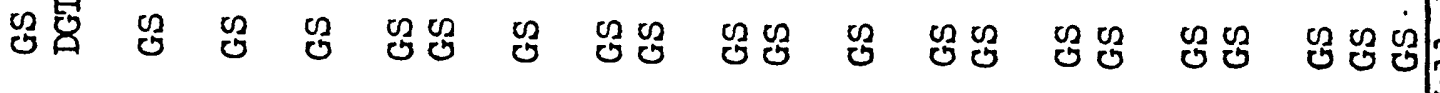

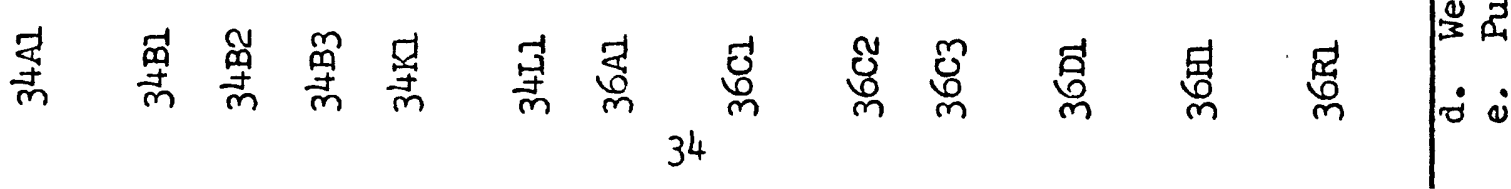




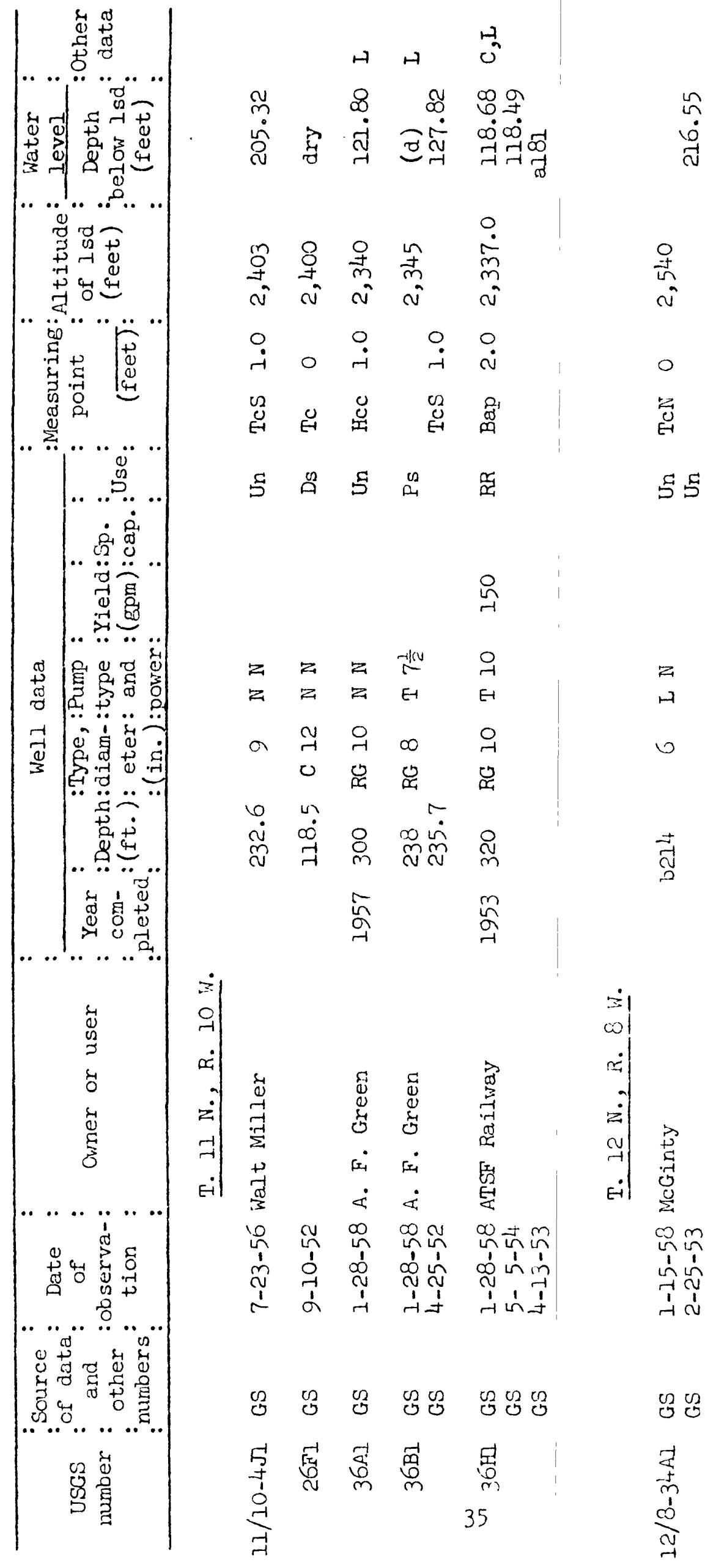


क ज ए

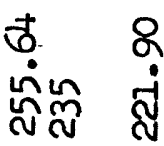

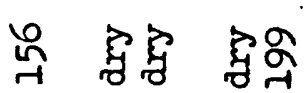

$\stackrel{2}{\infty}$

品 $\frac{m}{m}$

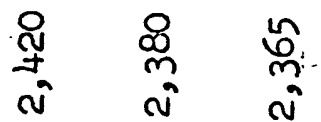

in in

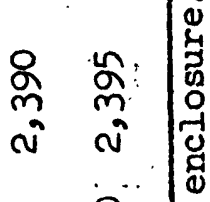

i 0

$\stackrel{0}{i} \quad \dot{i}$

㗲 学

U

i

U

总䔡

\& 5

\&



\&

$\vec{m}$

$m_{m}^{\infty}$ 鬲

in

이

$z \quad z \quad z$

$\underset{-1}{6}$

$\rightarrow$

ल

옹

r

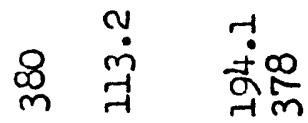

억 0

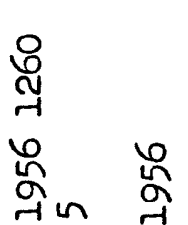

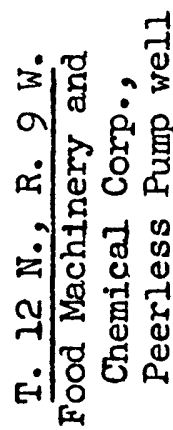

i.

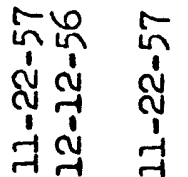

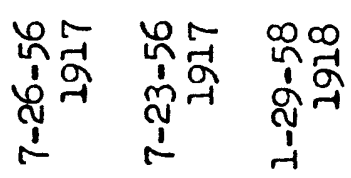

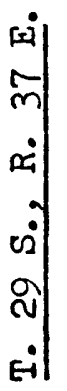

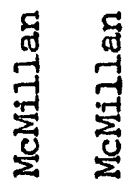

유

Z E

न

o 4

용 오ํ

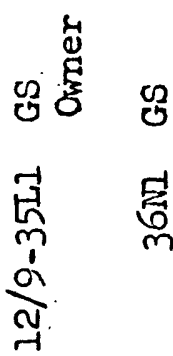

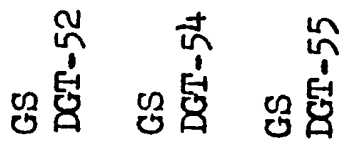

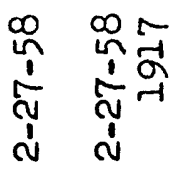
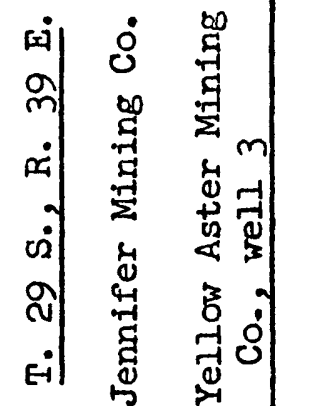

迎

秀 


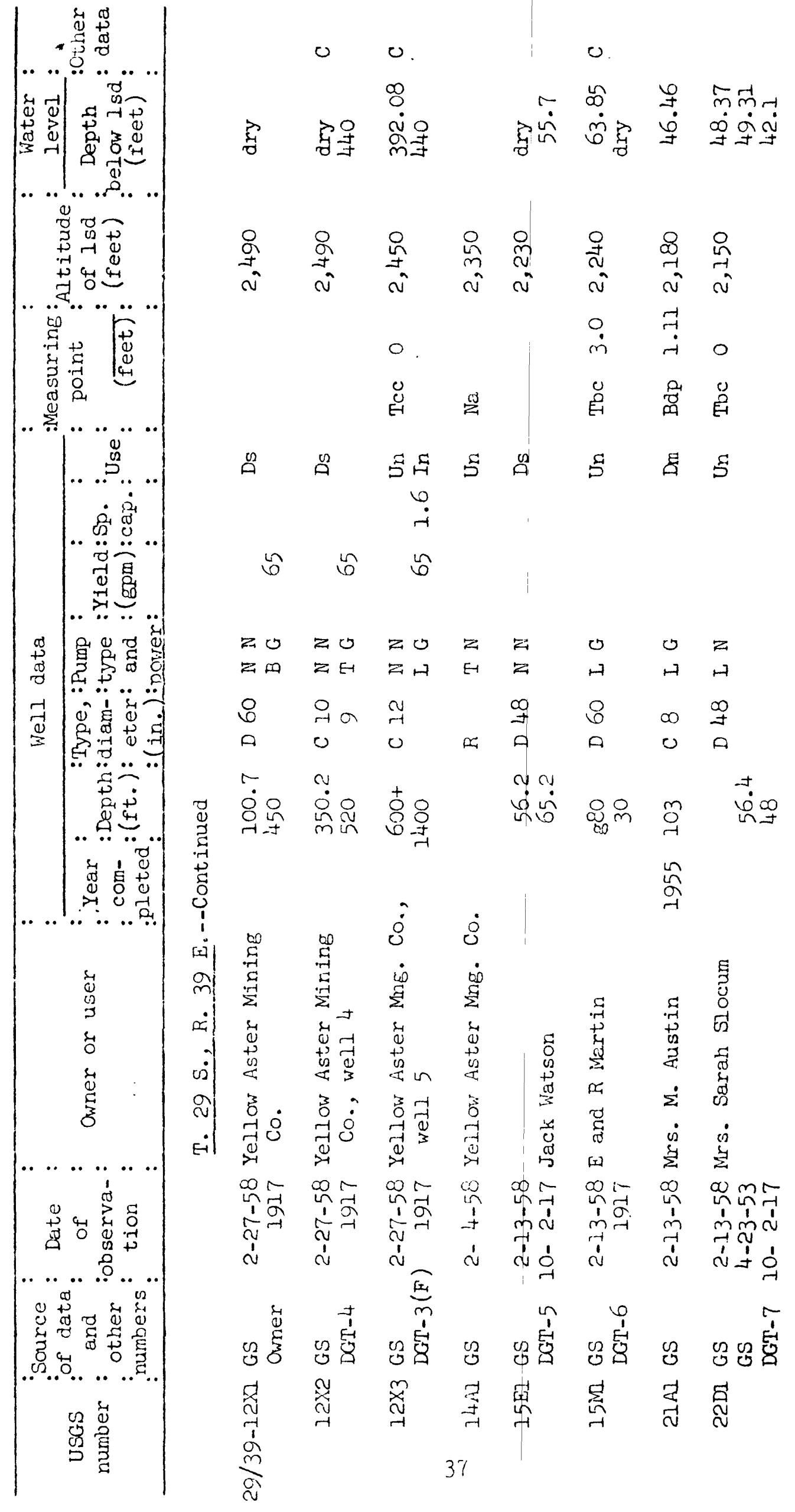




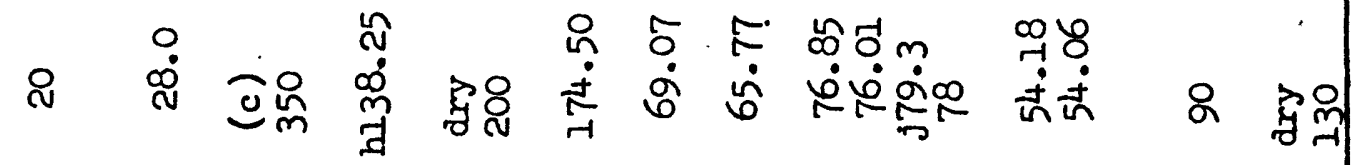

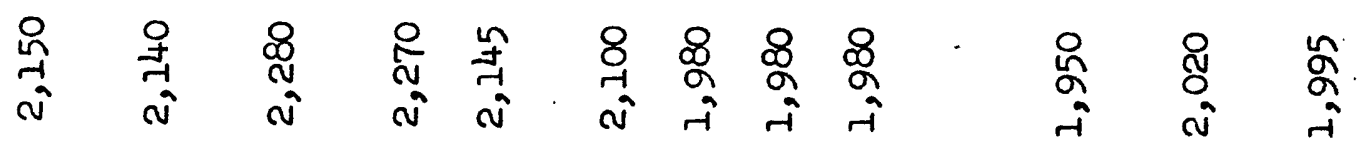

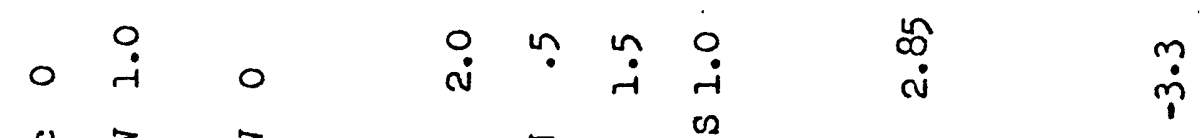

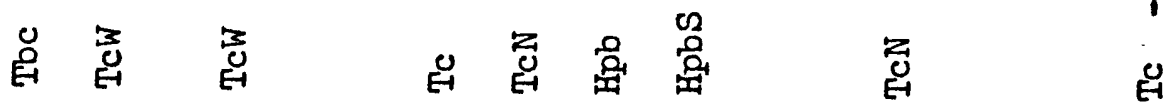

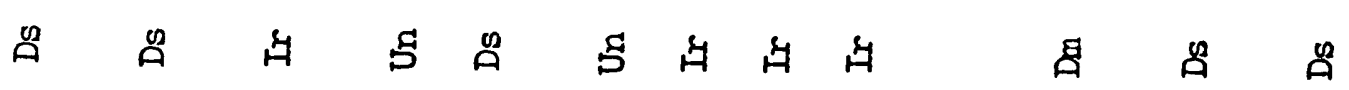

落

$B=0=2 \pi 000$

$B=E B Z Z B E A B$

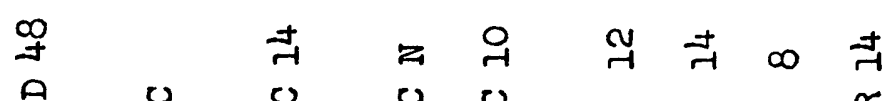

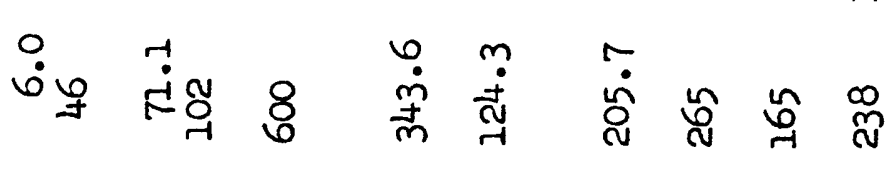
索参旁言

爵

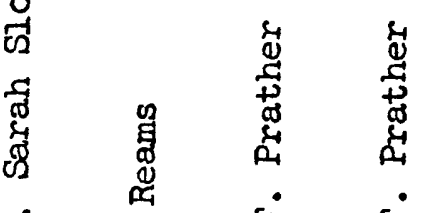

s

\& सं सं

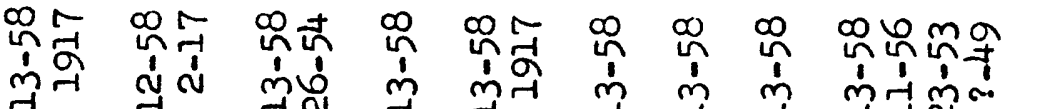

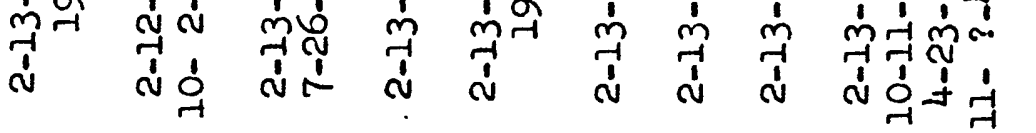

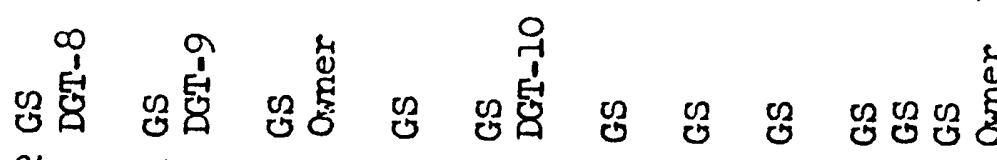
สั

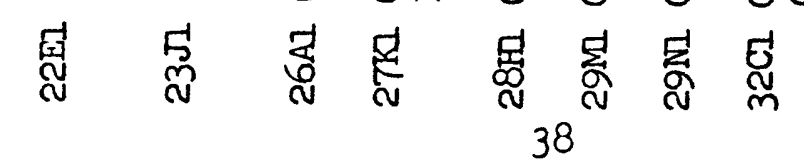

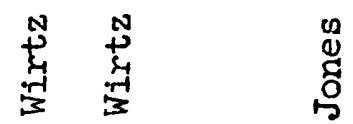

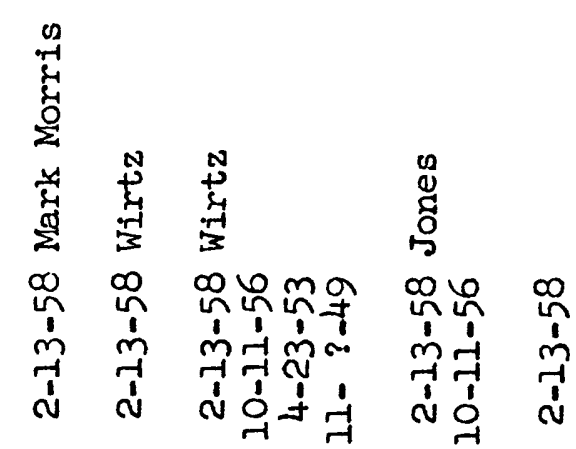

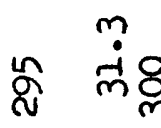

$\dddot{\dddot{q}}$

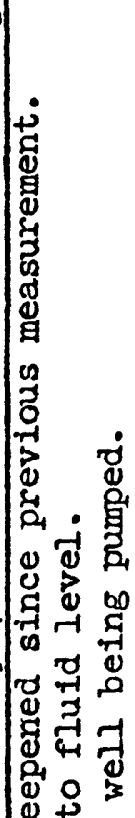




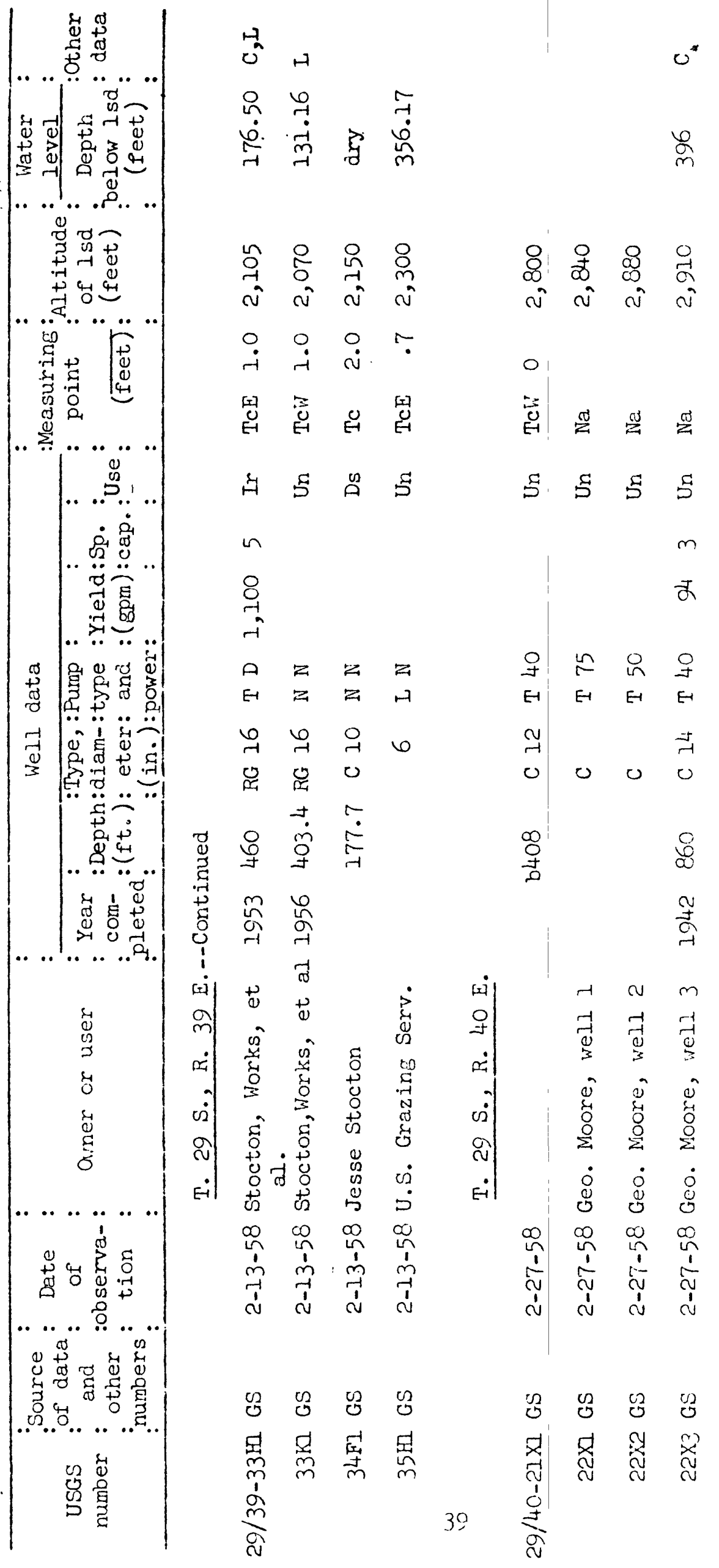




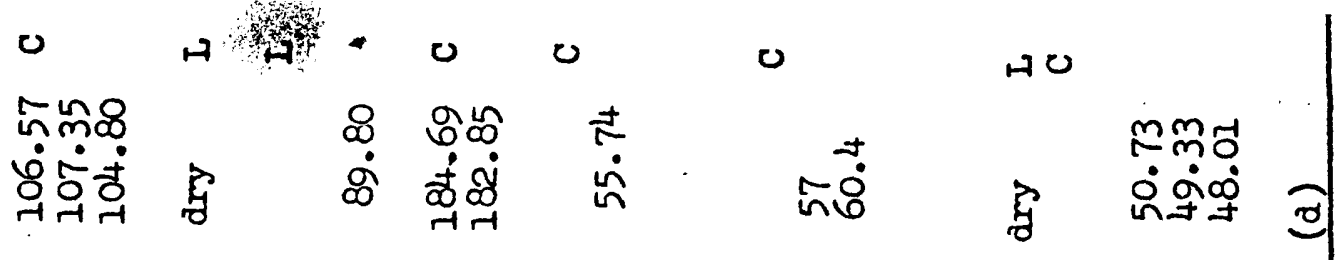

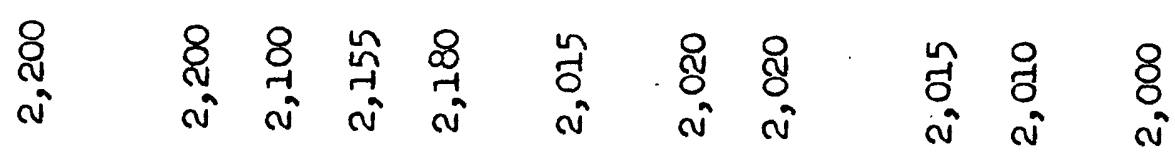

i $\quad r \quad \stackrel{i}{i} \stackrel{i}{i}$

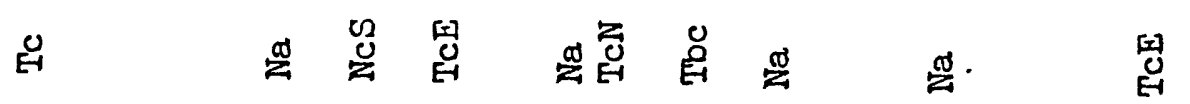

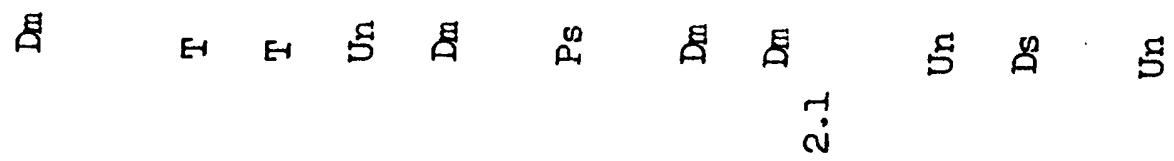

$\infty$

in

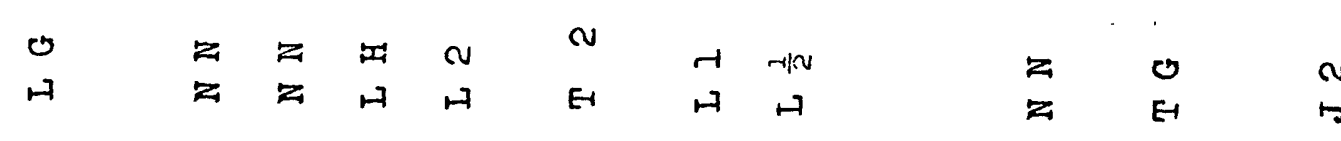

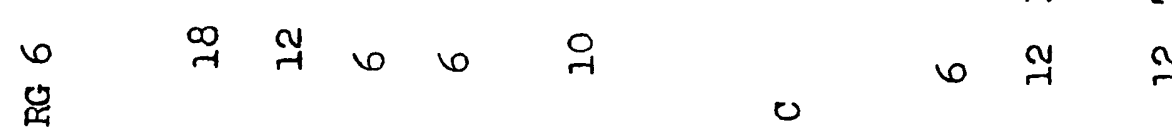

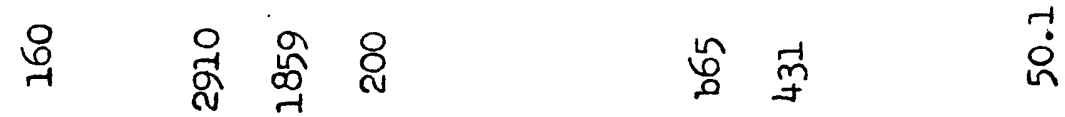

के

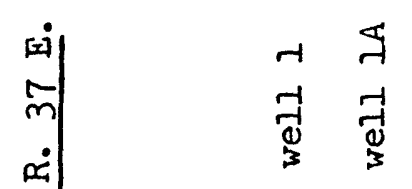

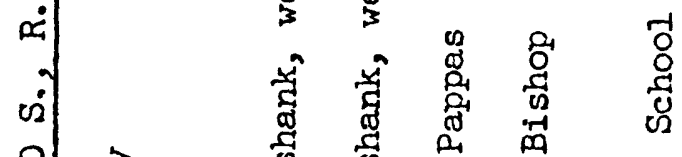

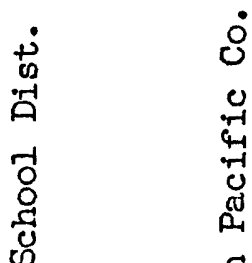

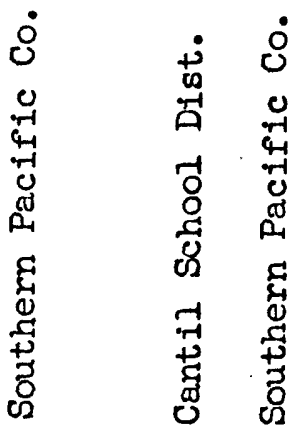

iํ류

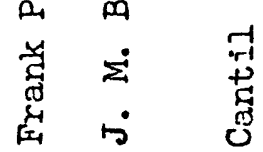

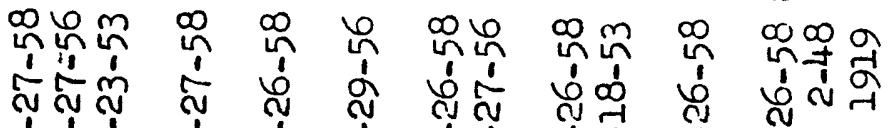

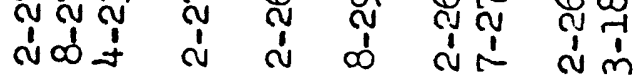

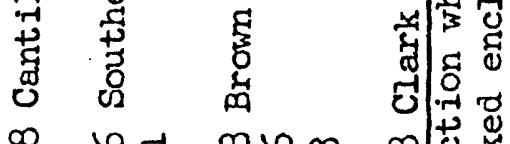

요

4

$\infty$

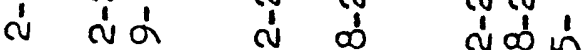

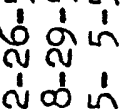

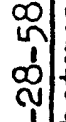

द्व ठ

(

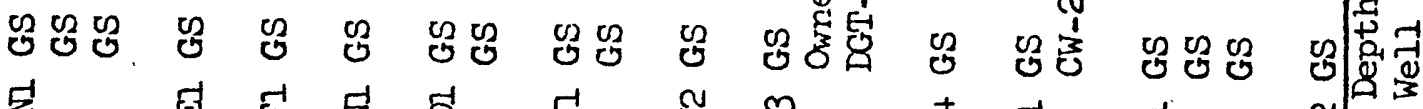

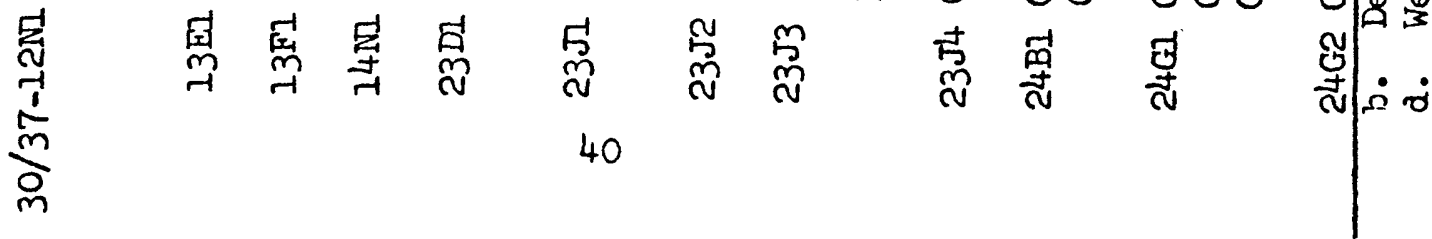




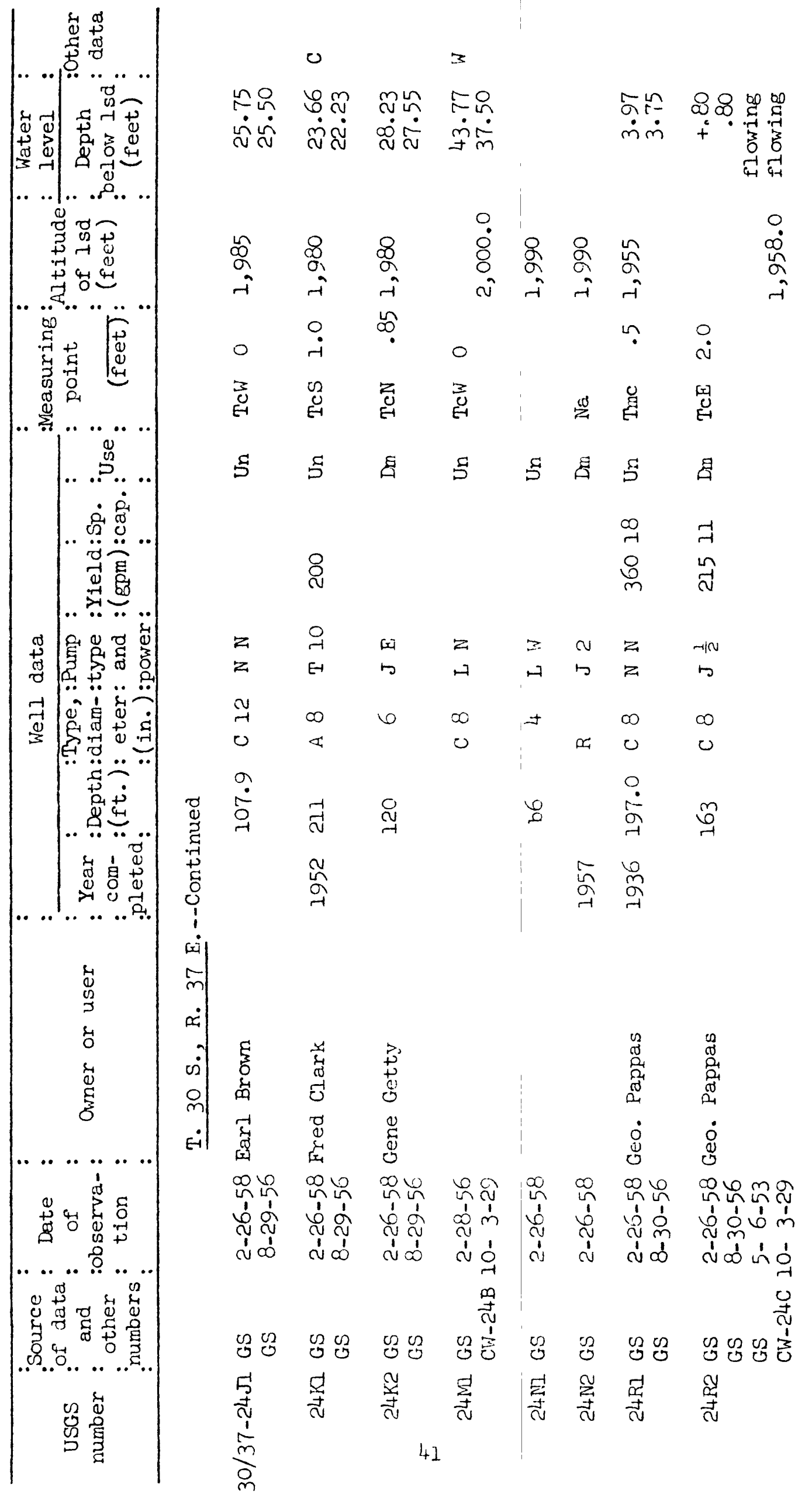


$\rightarrow \quad 0 \quad 3$

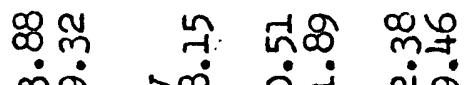

ஸ்ष

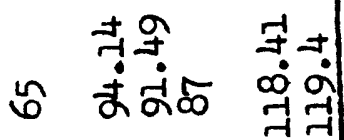

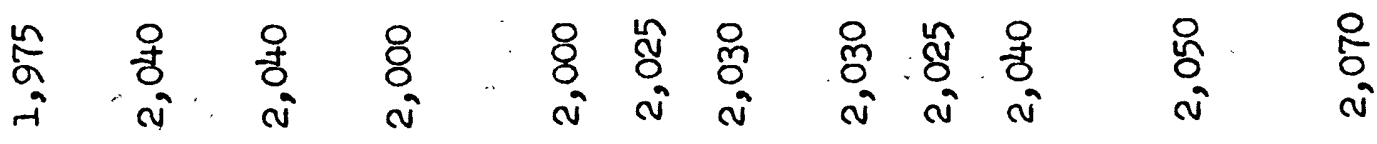

@ִ

鄯

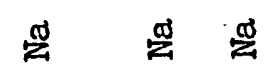

$\stackrel{\circ}{i} \stackrel{i}{i}$

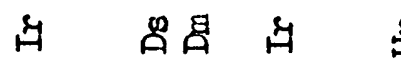

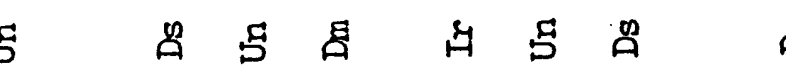

总 总

คำ

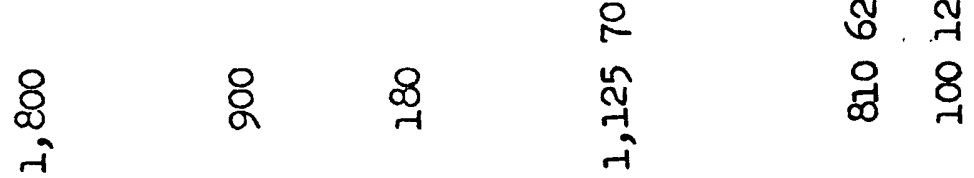

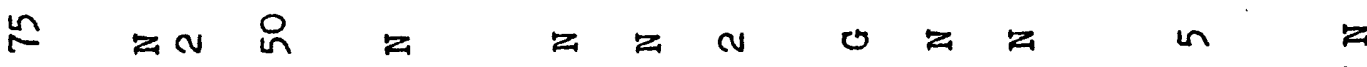

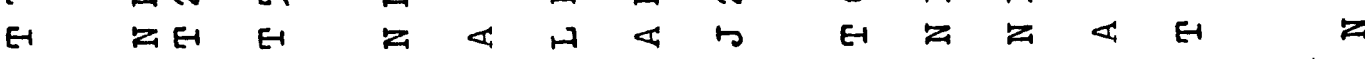

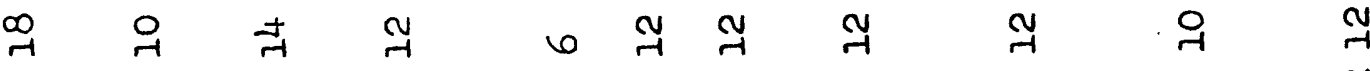

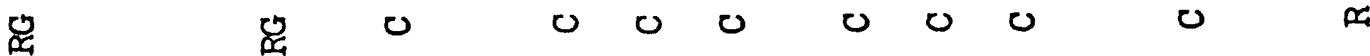

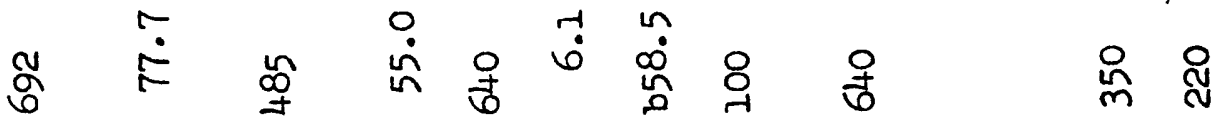

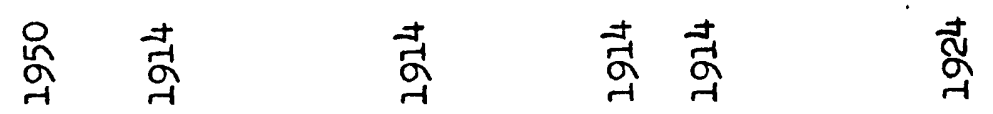

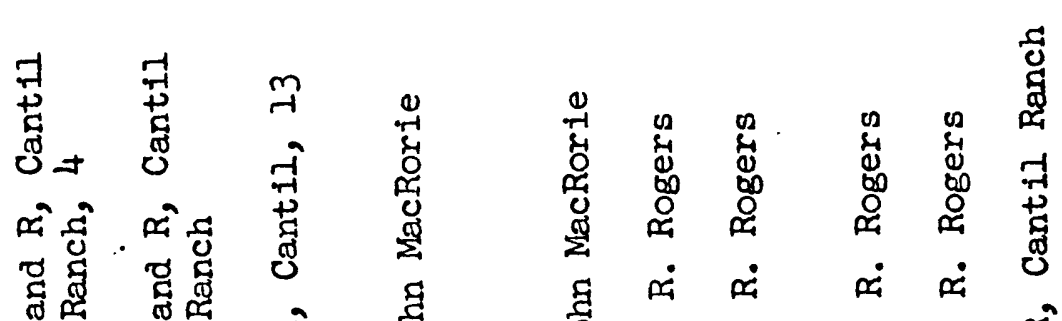

द्ध

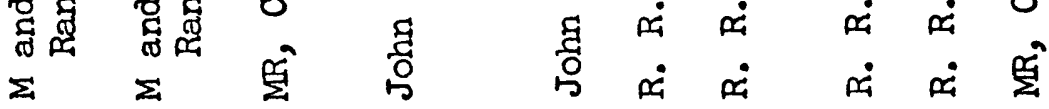

mm

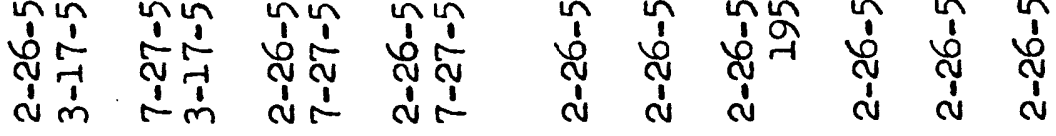

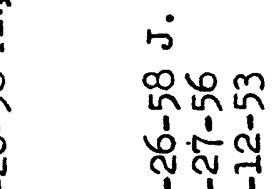

í

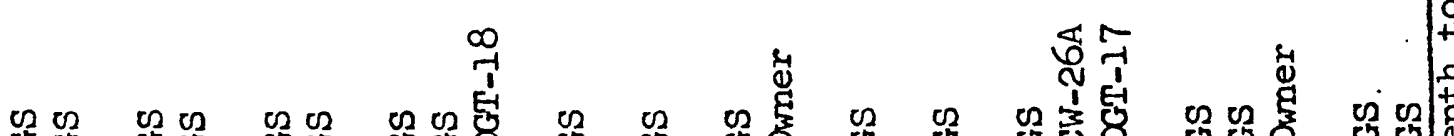

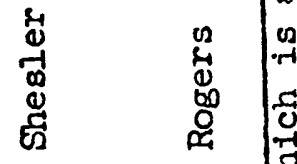

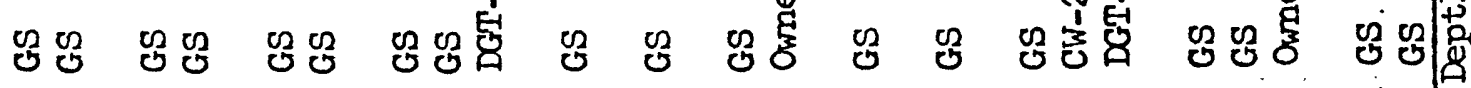

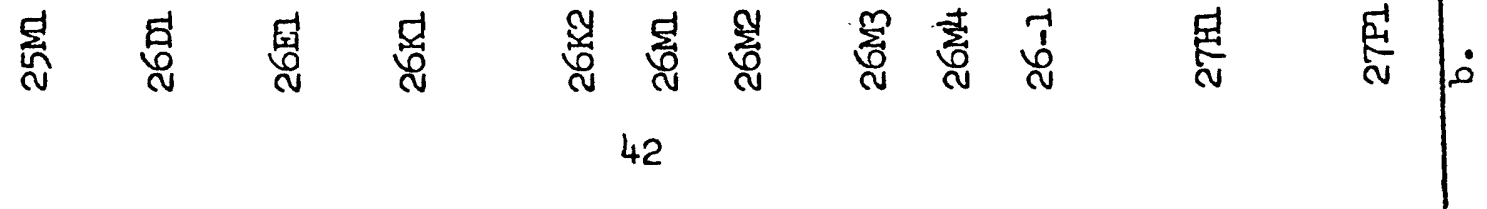




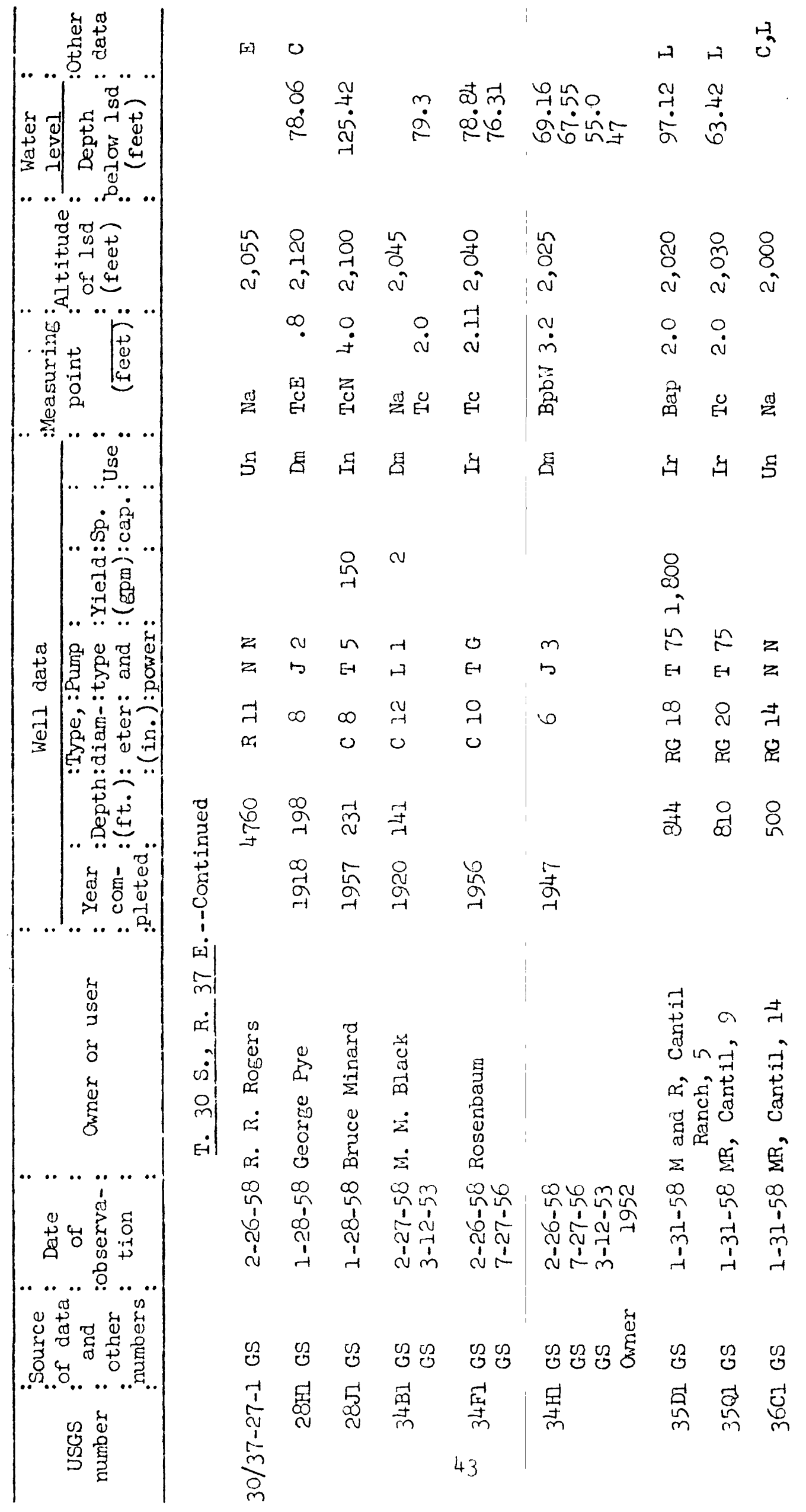


H H

กีल

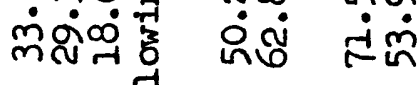

00

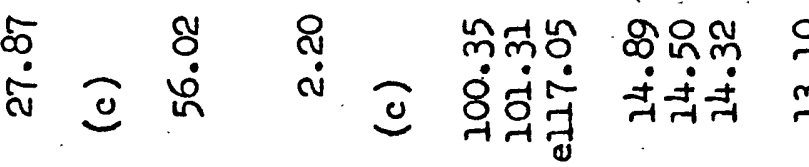

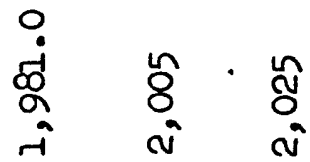

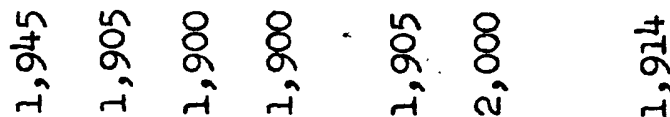

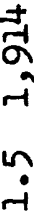

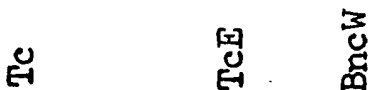

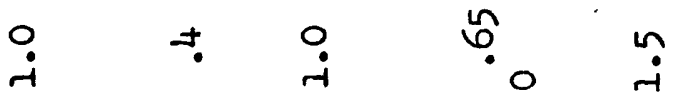

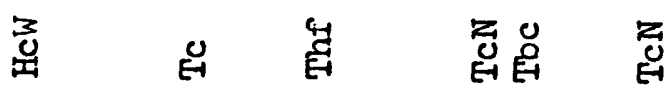

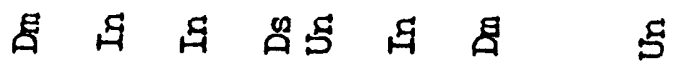



오

8

8

-1

กิ

n $n$ in

E $\rightarrow$ E

न

ल ए ए

$\stackrel{\infty}{a}$

กิ

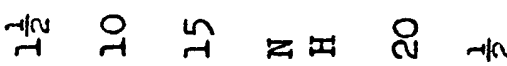

E E E ZHE H

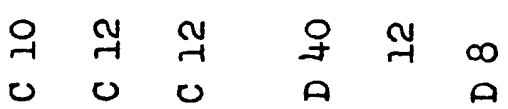

a

옥

$\stackrel{p}{\dot{m}}$

वे

志

$z$

$\underset{-1}{\Upsilon}$

z

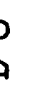

r $\cong$ 아

ఫิ

离

sa

舀参

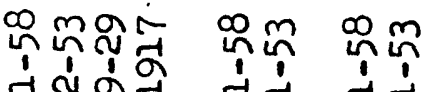

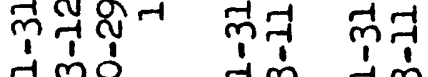

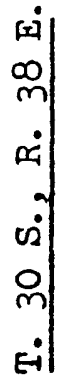

$\dot{0} \quad \dot{0} \quad \dot{0} \quad \dot{0} \quad 8$

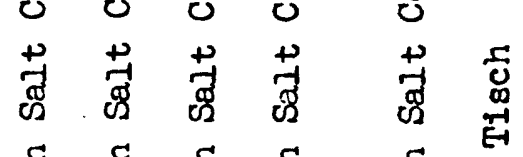

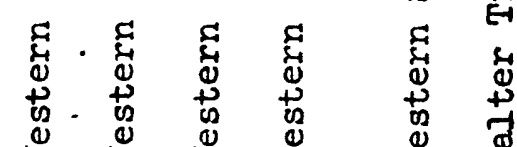

ए

ن

i i

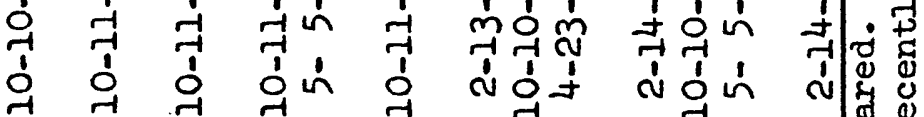

ఠิ

凹必它武

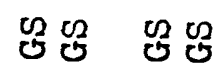

贷

鸸 点

ஜ ஜ

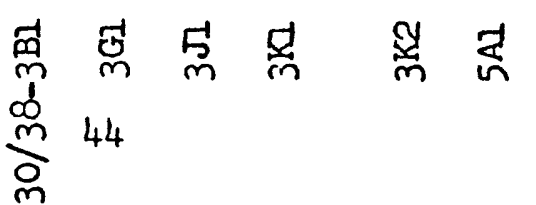

菖 


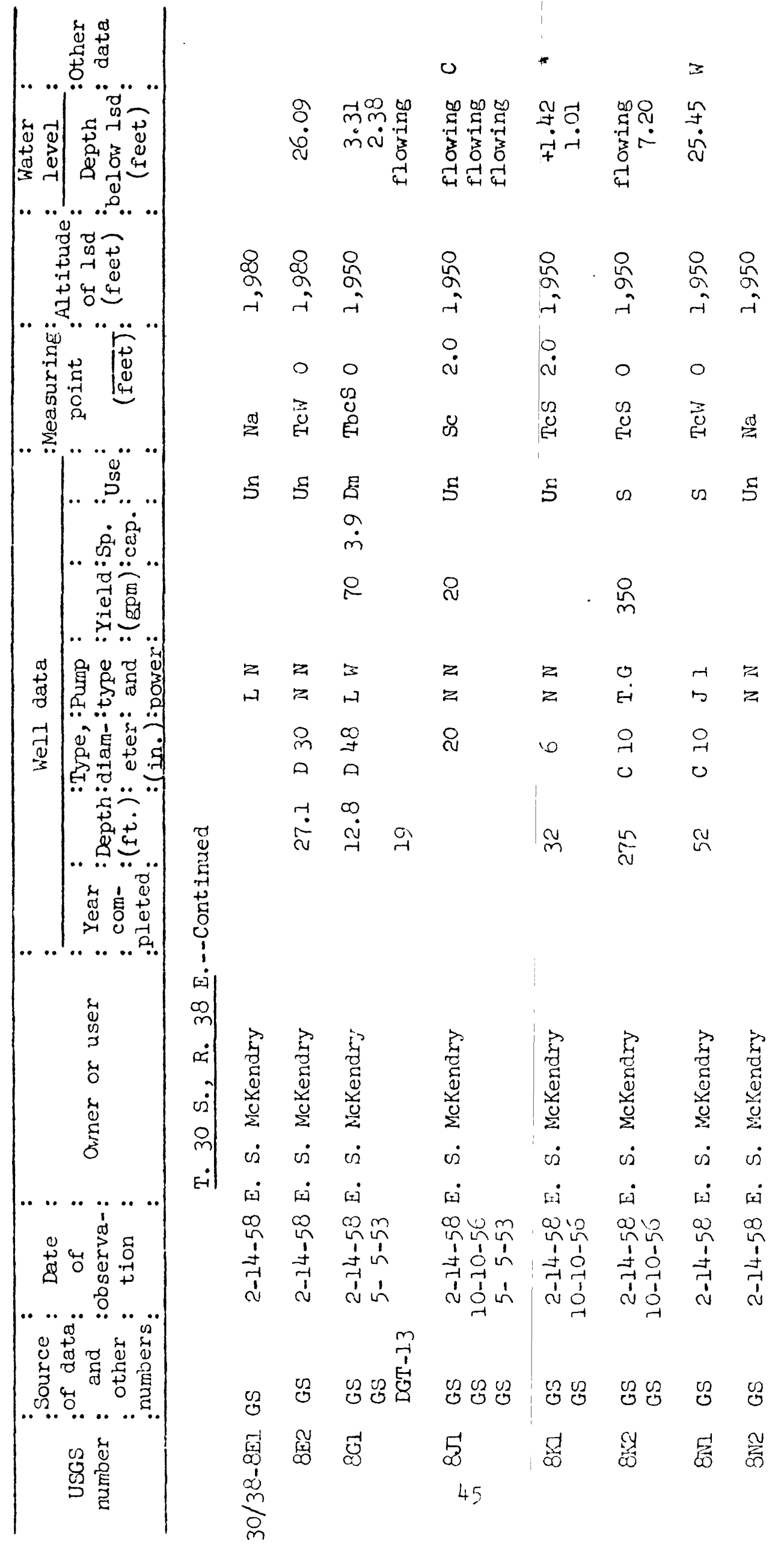




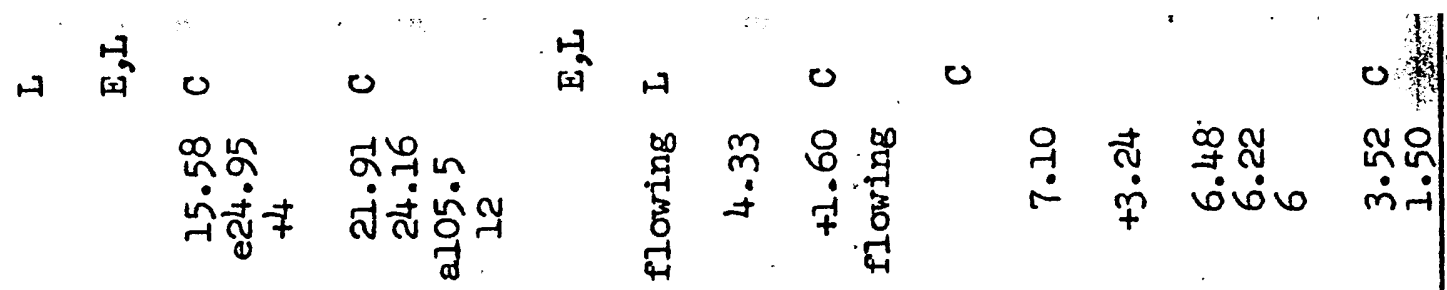

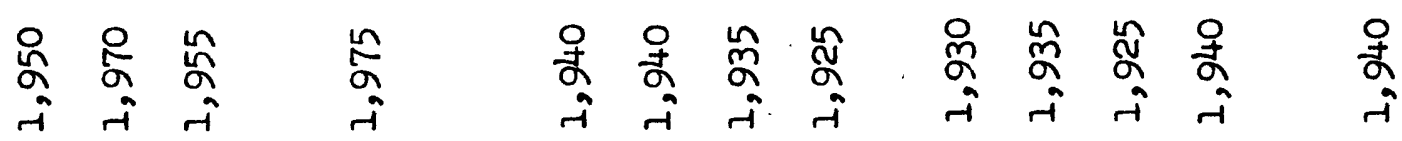

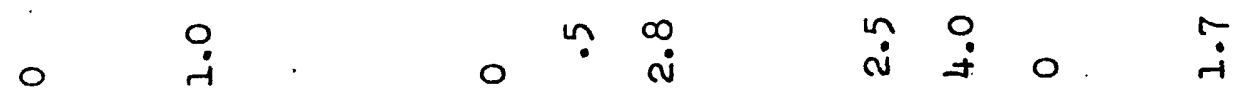

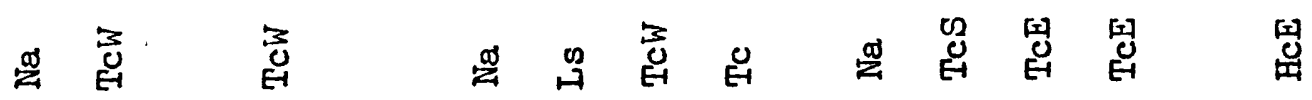

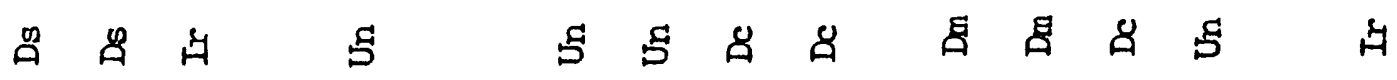

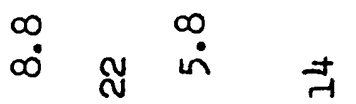

品 $\underset{i}{\stackrel{4}{7}}$ \&

吕

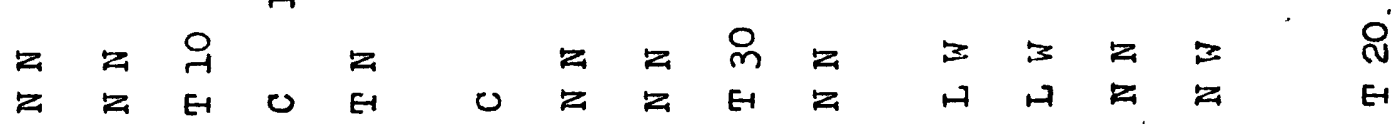

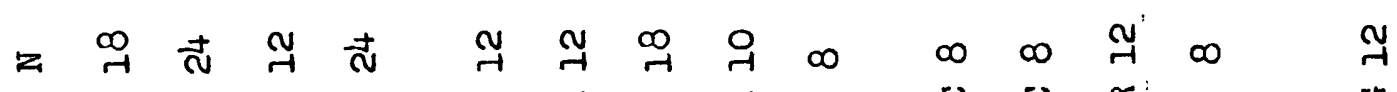

$\infty \begin{array}{llllllll}0 & 0 & 0 & \infty & 0 & 0 & 0 & \infty\end{array}$

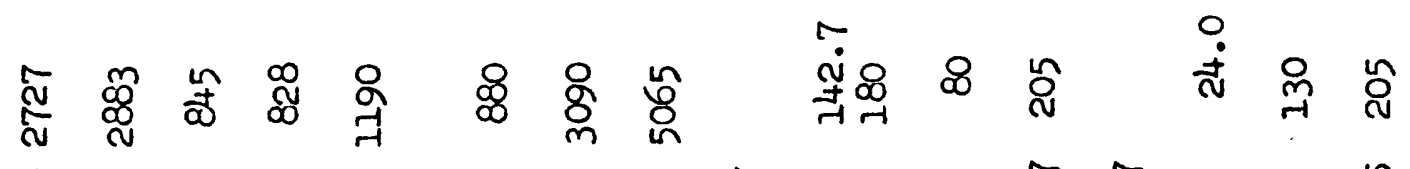

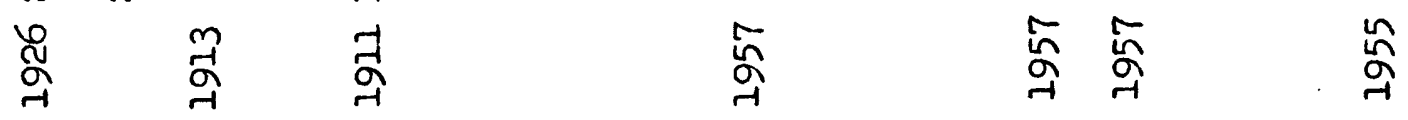

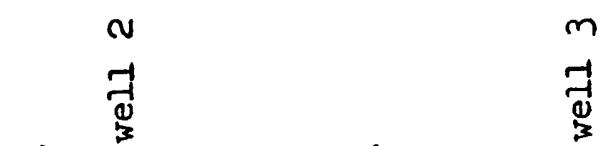

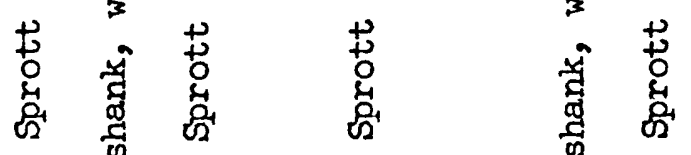

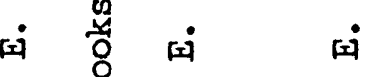

is क्ष $\dot{0}$ is

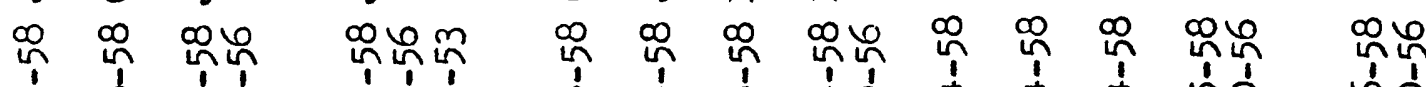

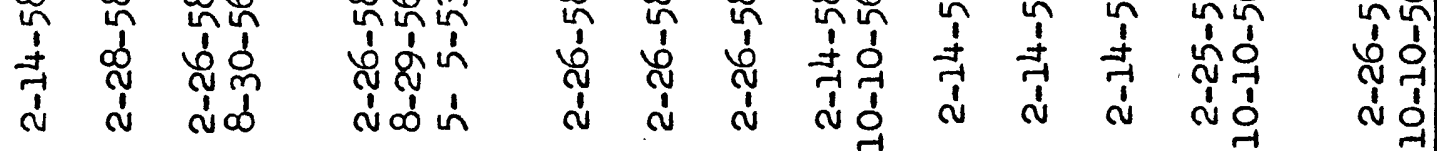

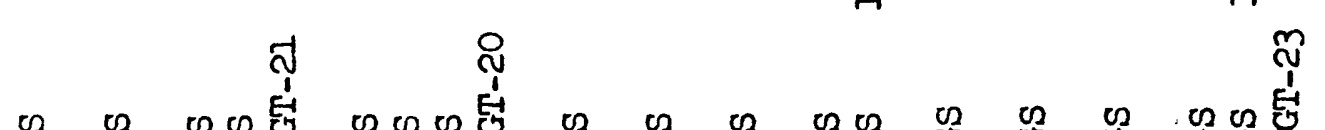

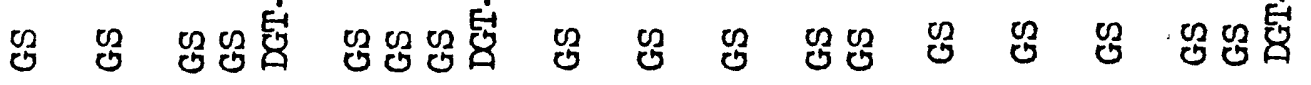

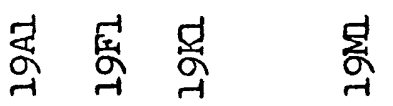

壱 芯 突 46 


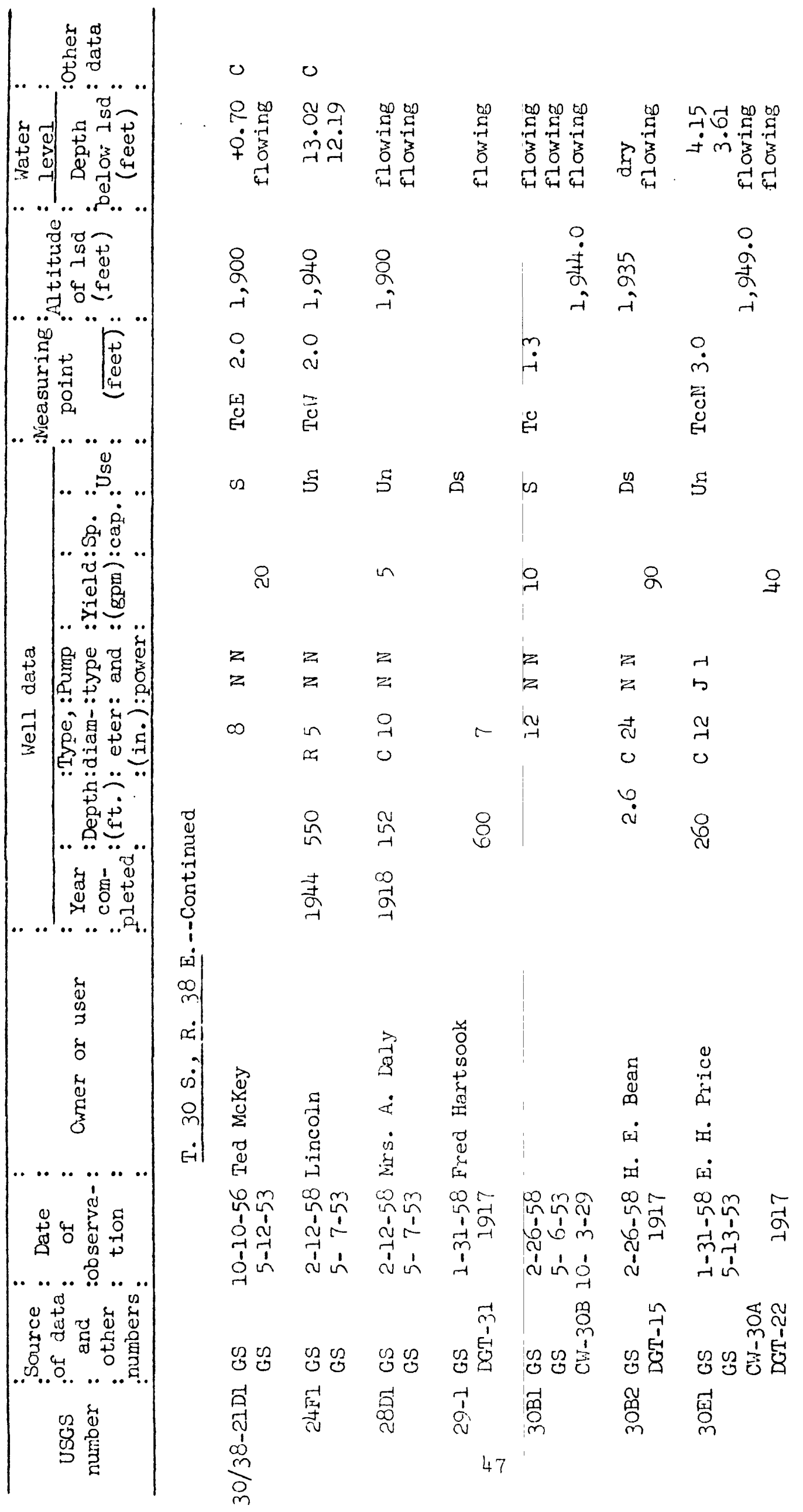




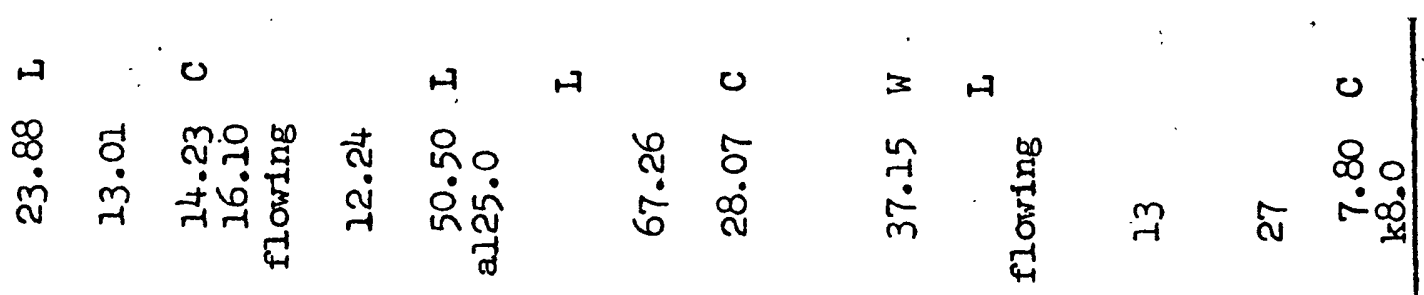

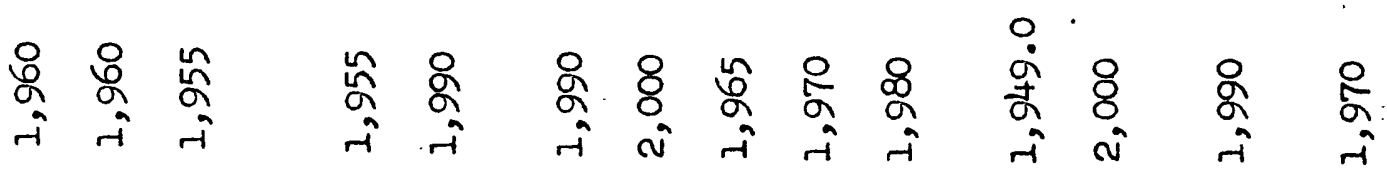

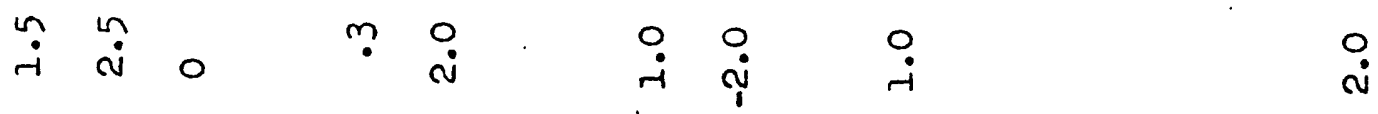

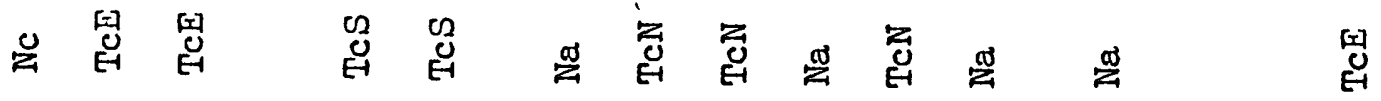

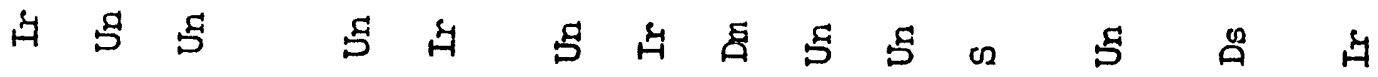

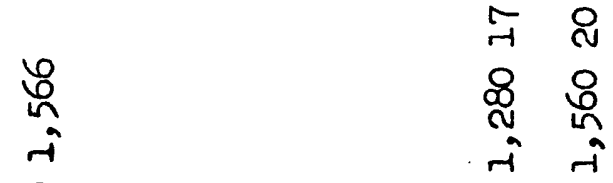

令

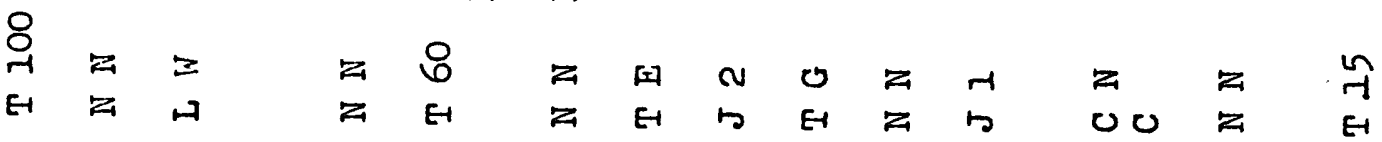

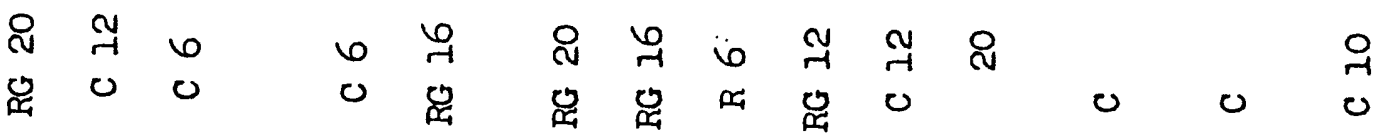

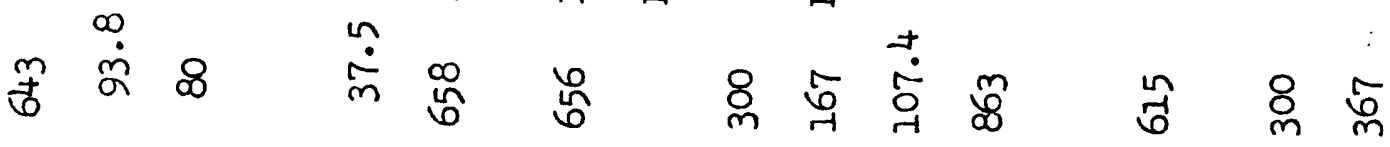

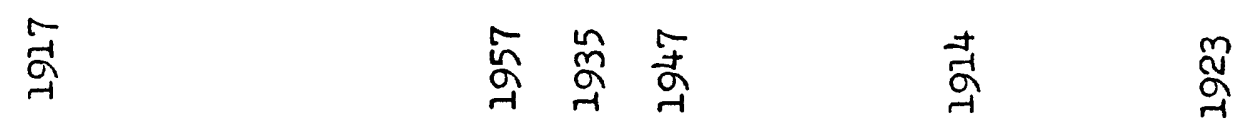

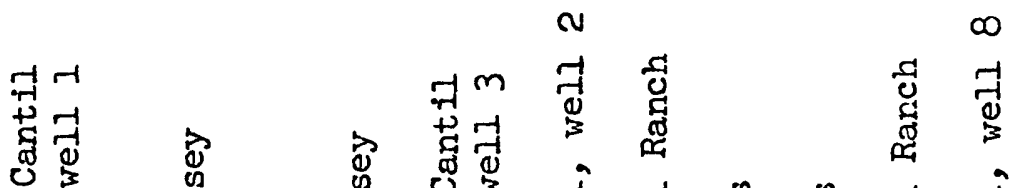

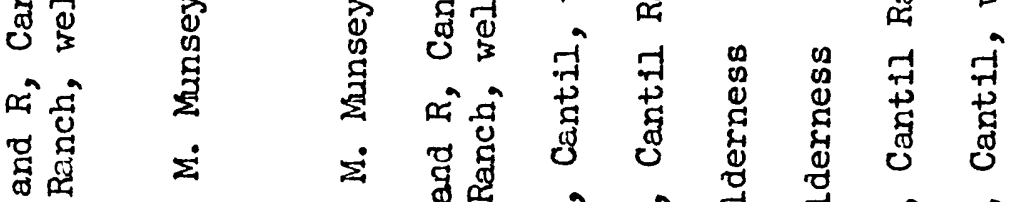

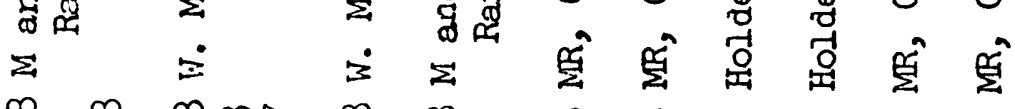

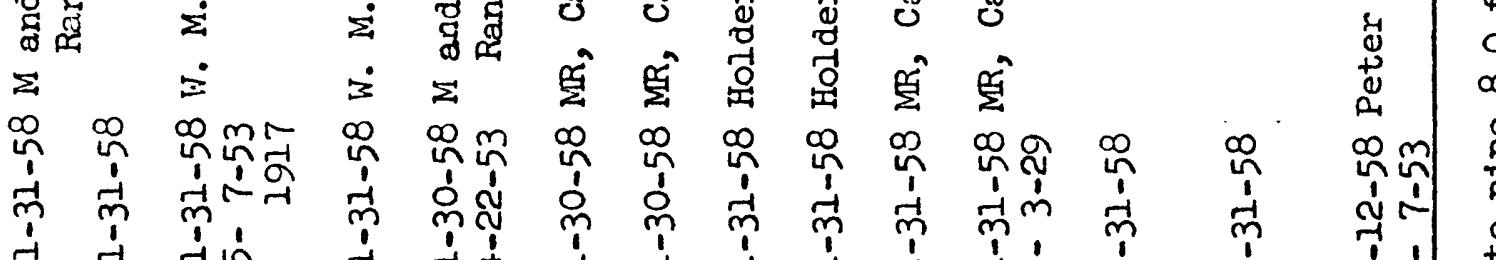

gु

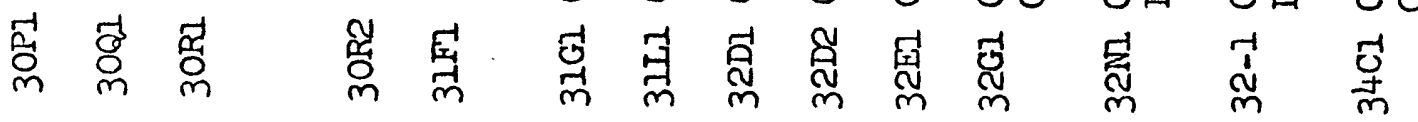




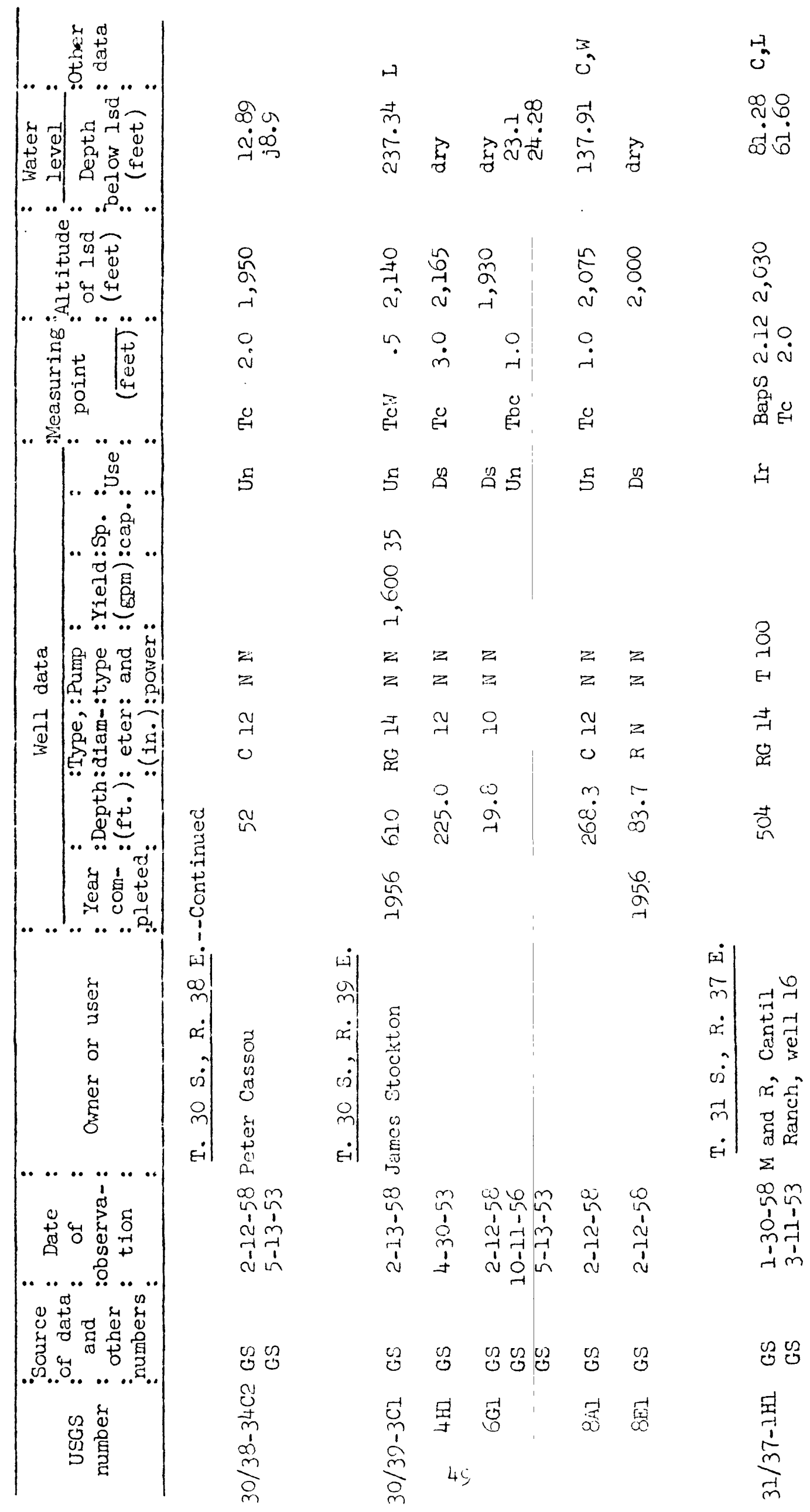




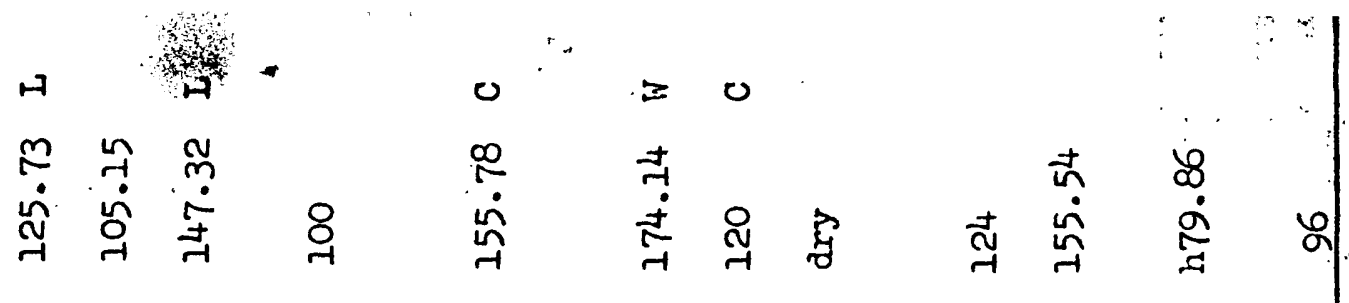

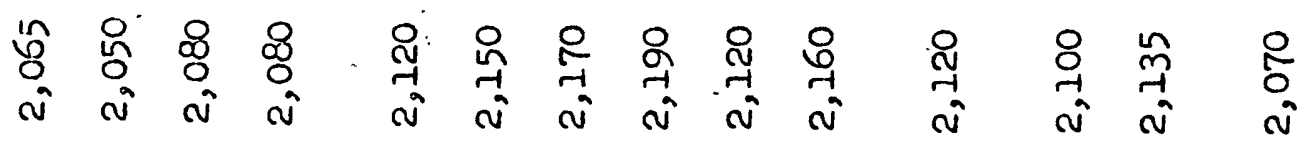

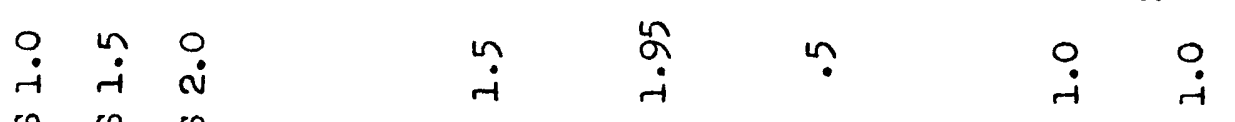

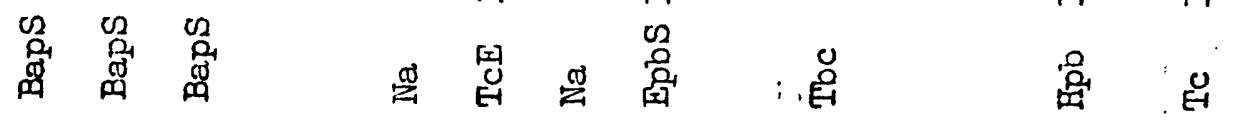

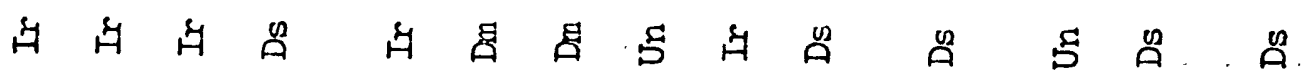

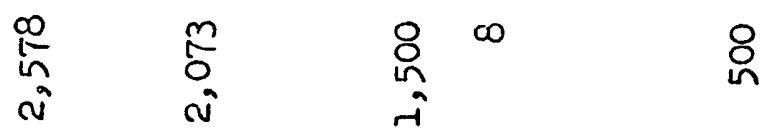

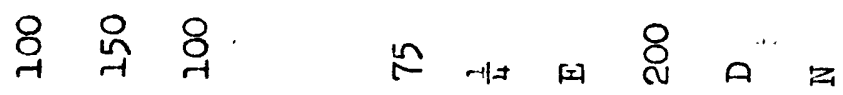

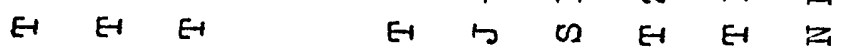

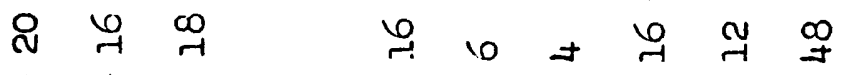

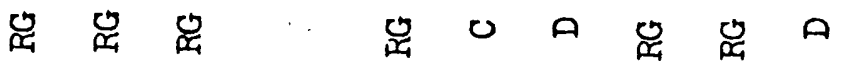

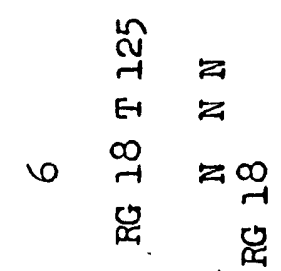

兽

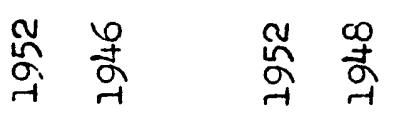

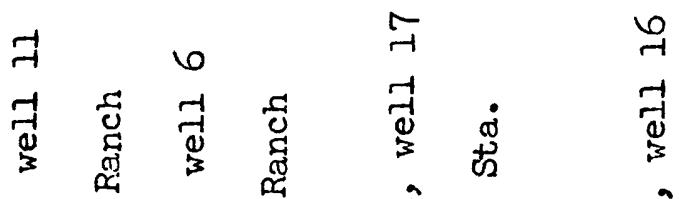

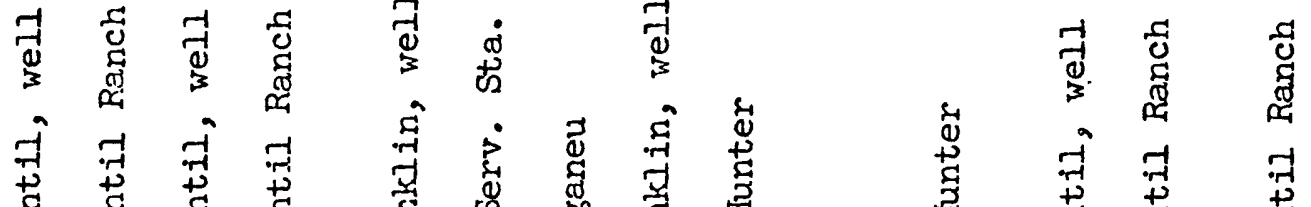

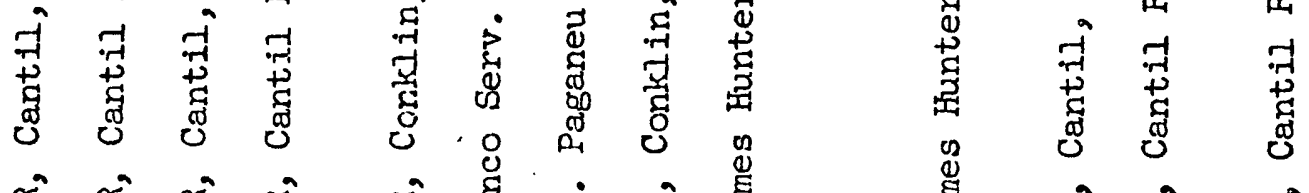

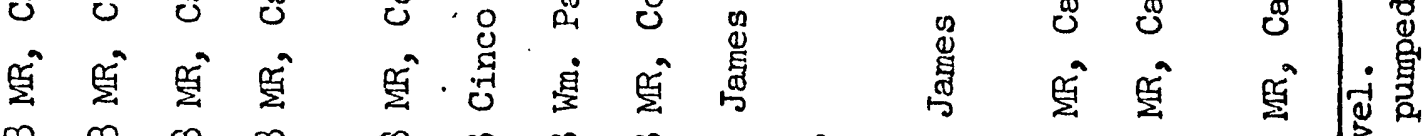

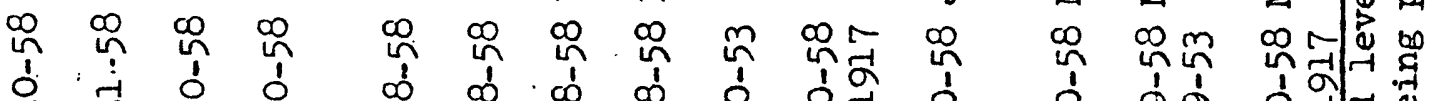

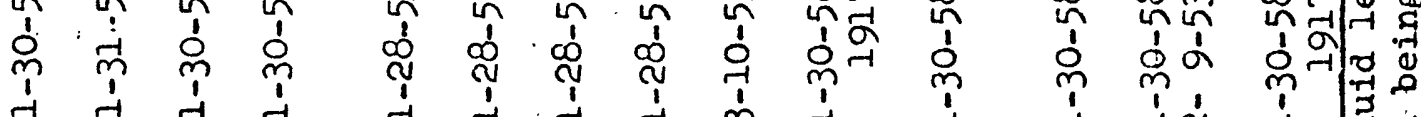

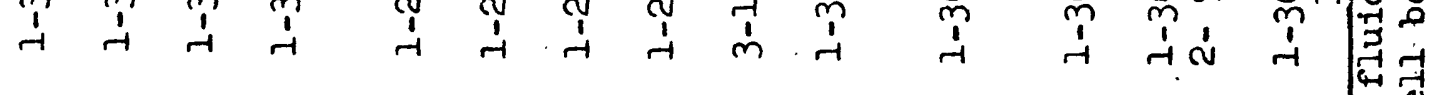

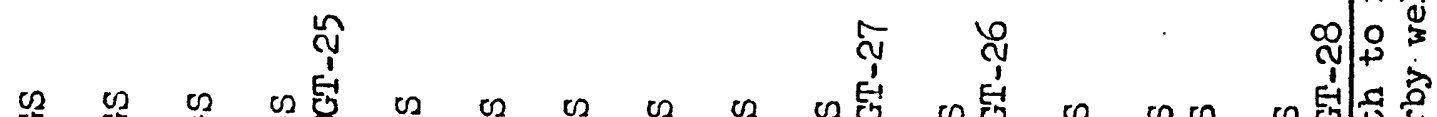

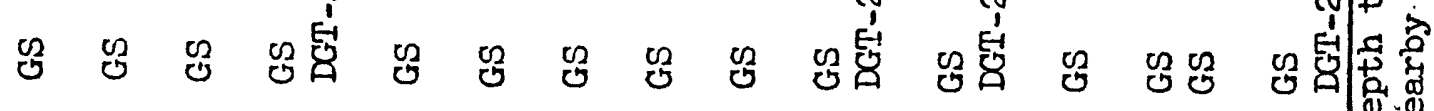

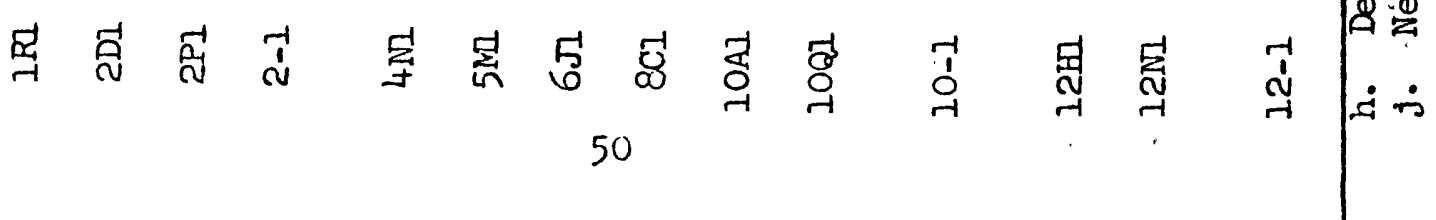




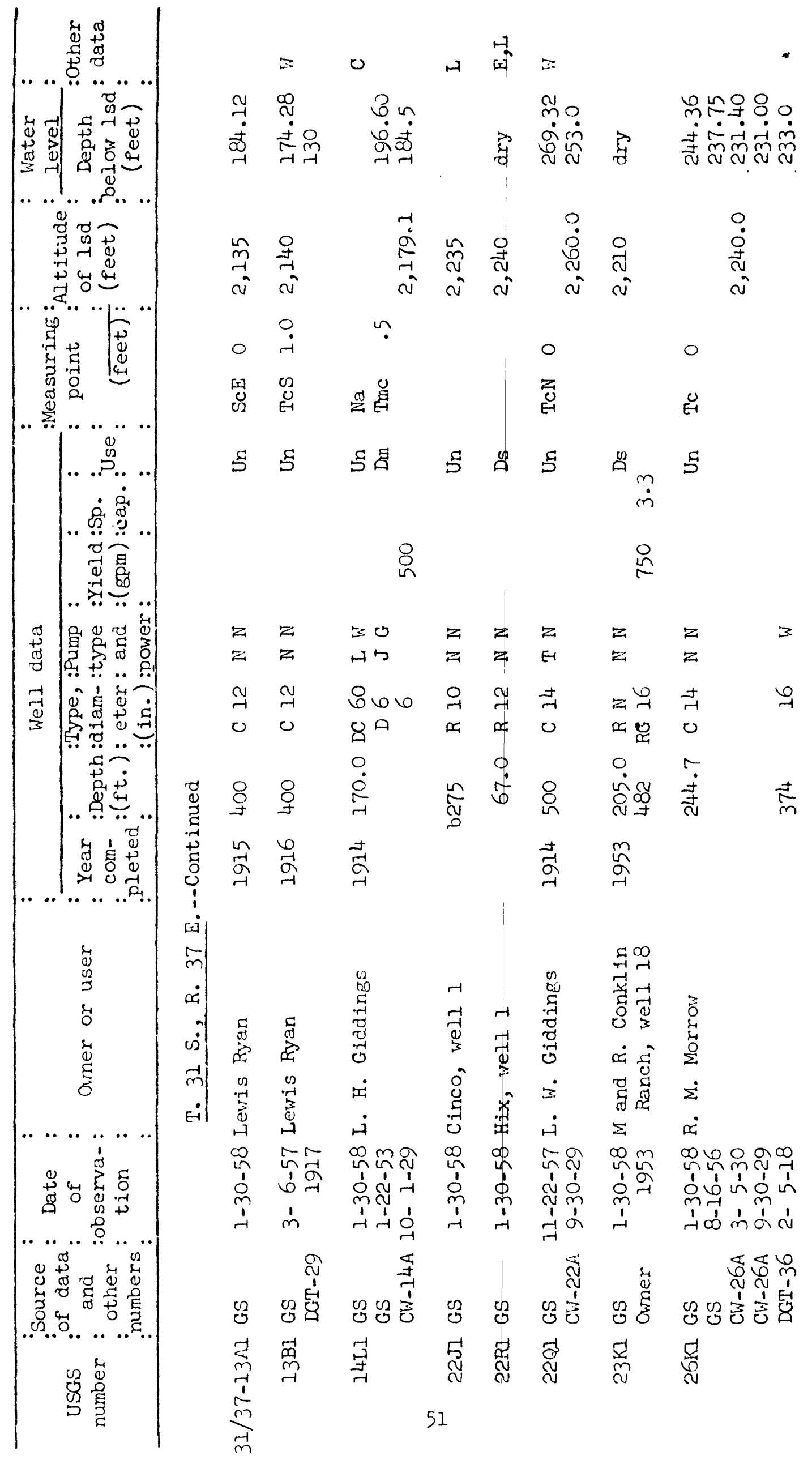


a 10

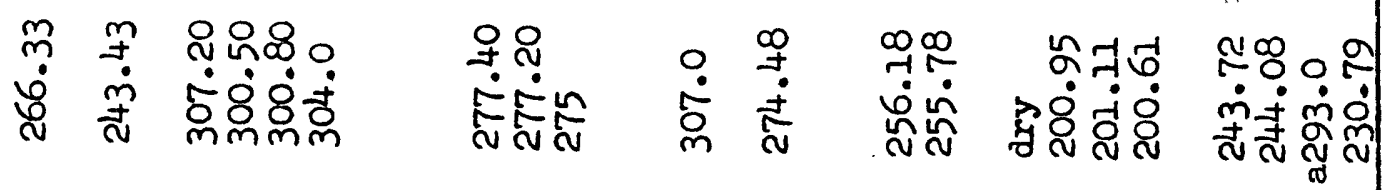

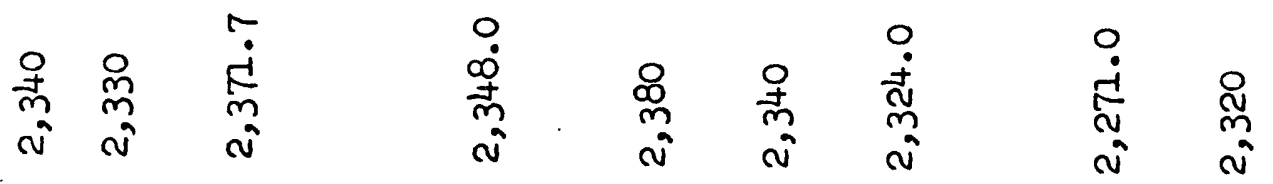

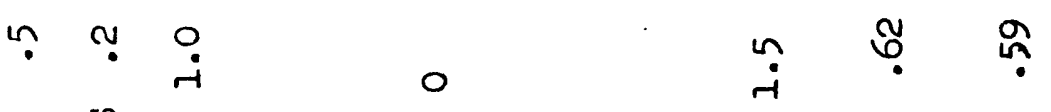

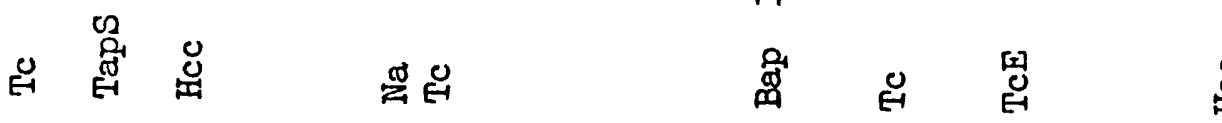

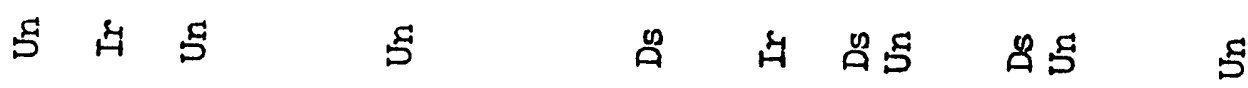

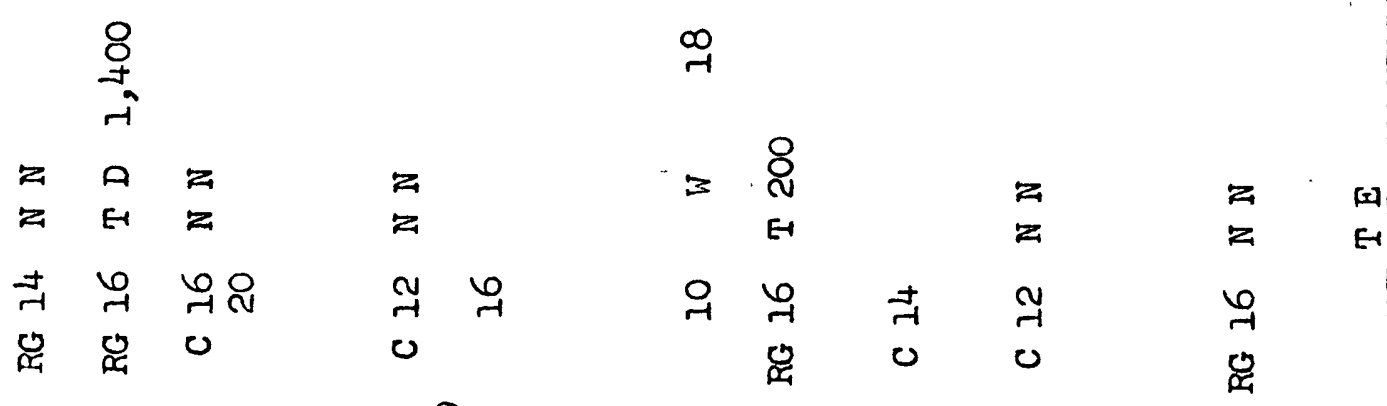

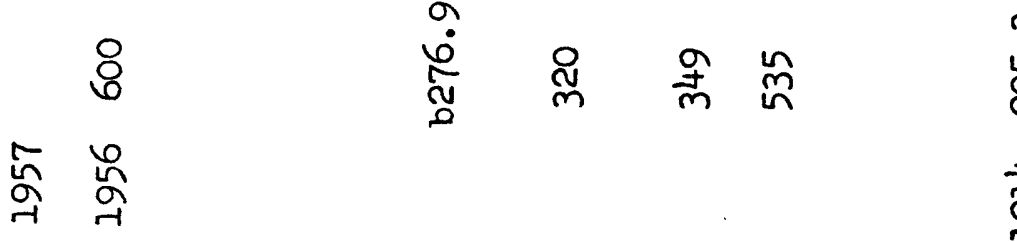

范落

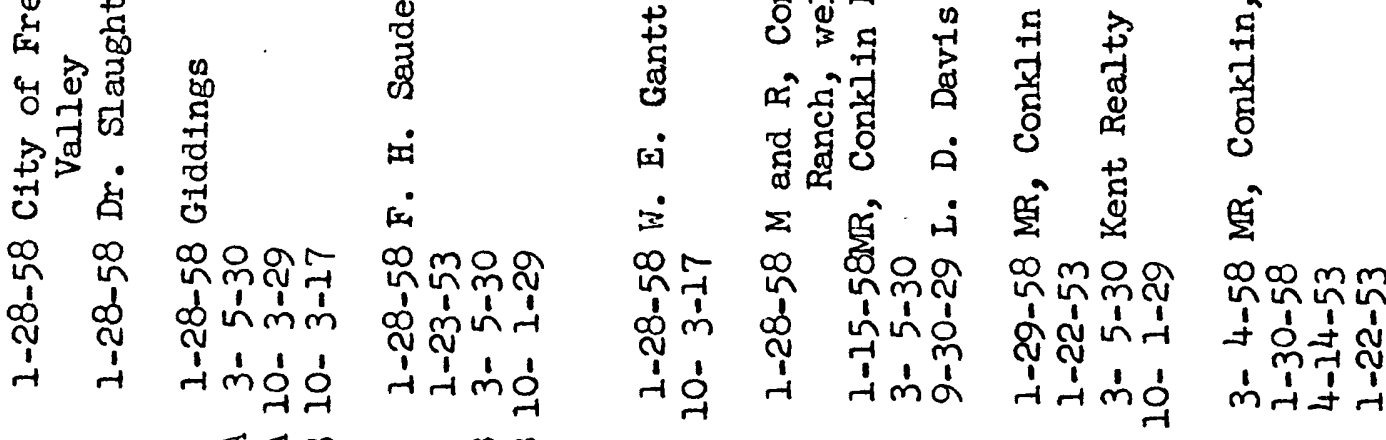

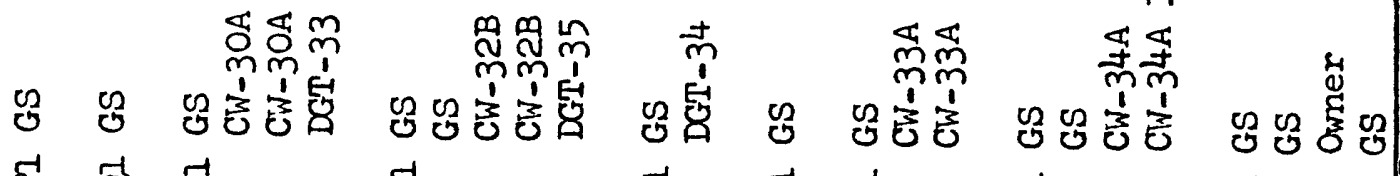
范 స్

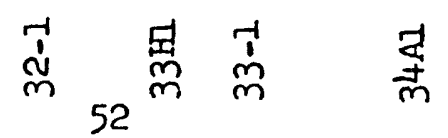
窟 


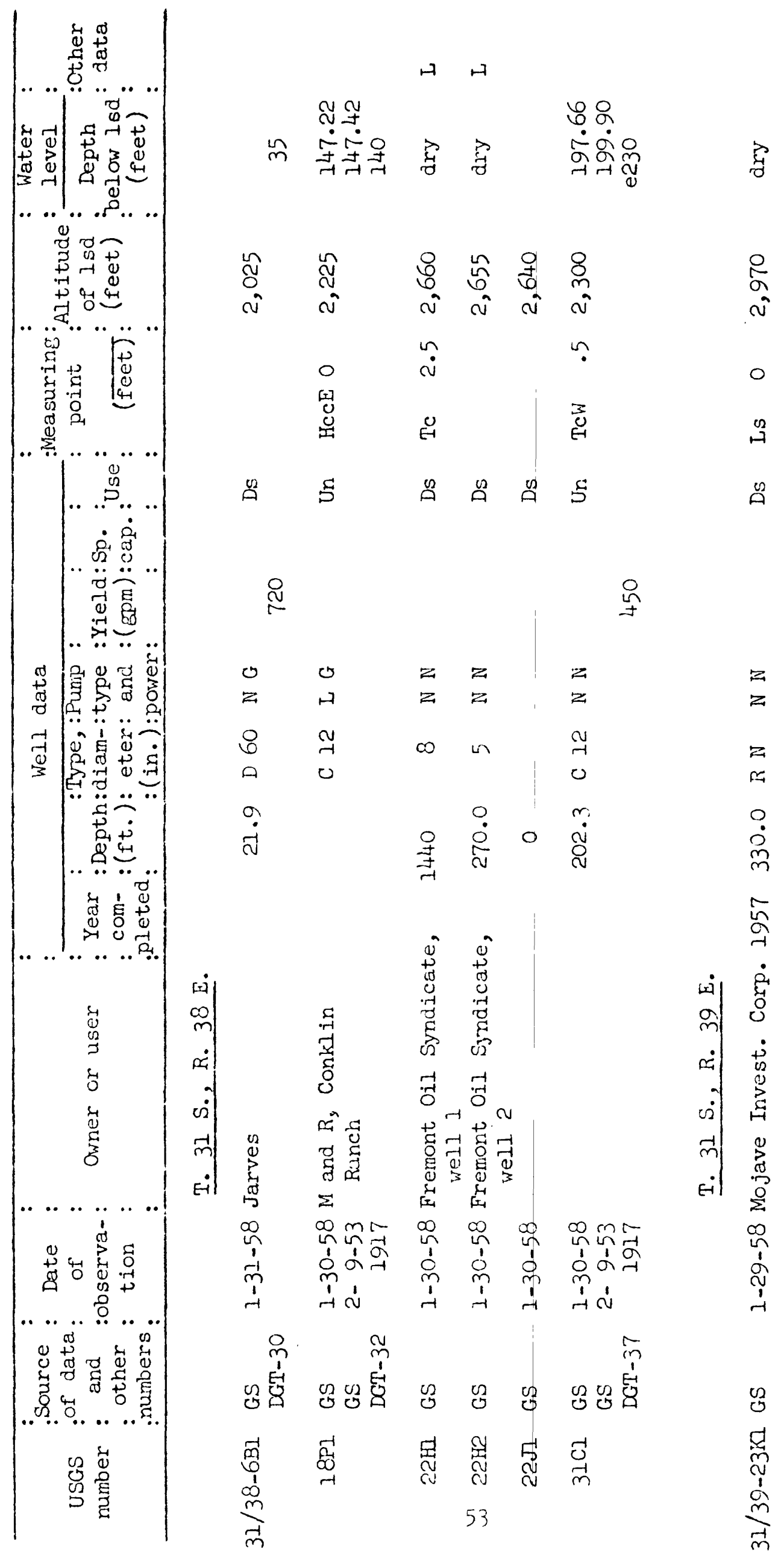




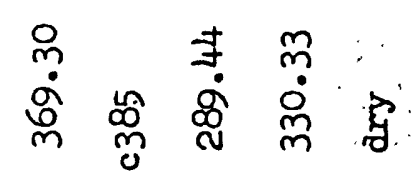

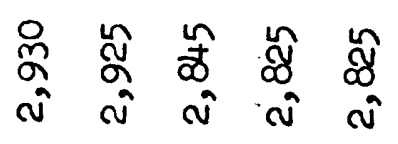

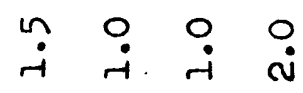

蛋品哭畓

5 5 5 5 5 ถ
บำ. 윰

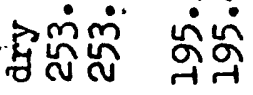

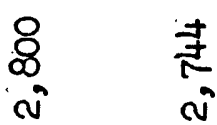

ฯ

ㅂ.

ค 2.5

봉

乌

㝵

م:

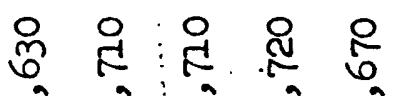

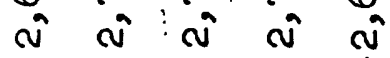

$\stackrel{n}{\rightarrow} \stackrel{i}{i}$

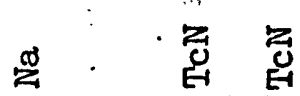

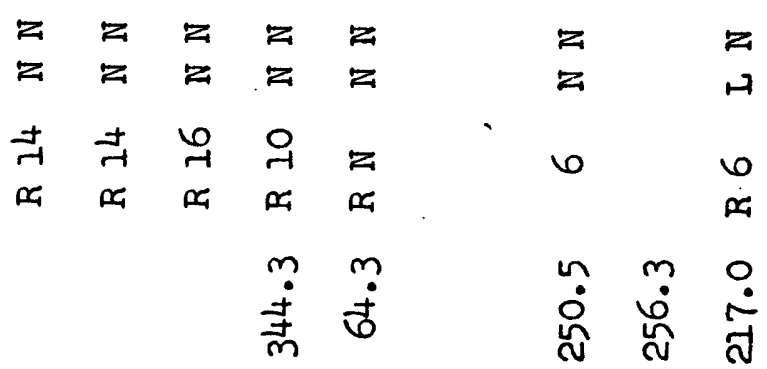

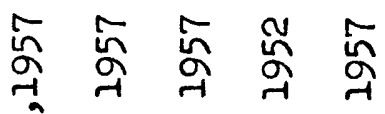

ठั

苟

要表

을 의

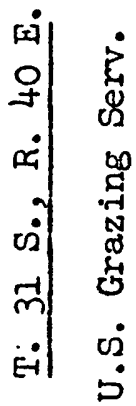

蛋

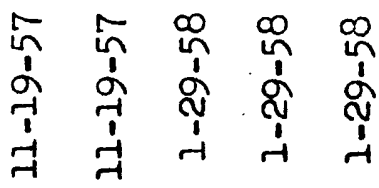

$\dot{z}$

กำำ ing

ล้กิ่

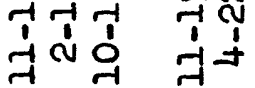

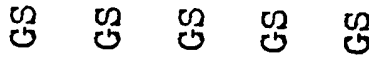

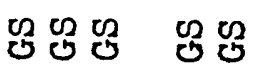

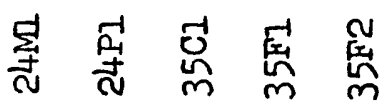

衵 54 总
吕星 नो 5

$z$ N $z=z$

乙

$\begin{array}{lllll}8 & 0 & 6 & 0 & 9\end{array}$

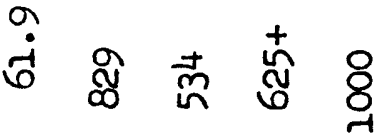

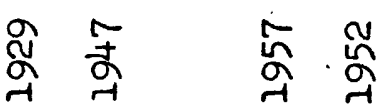




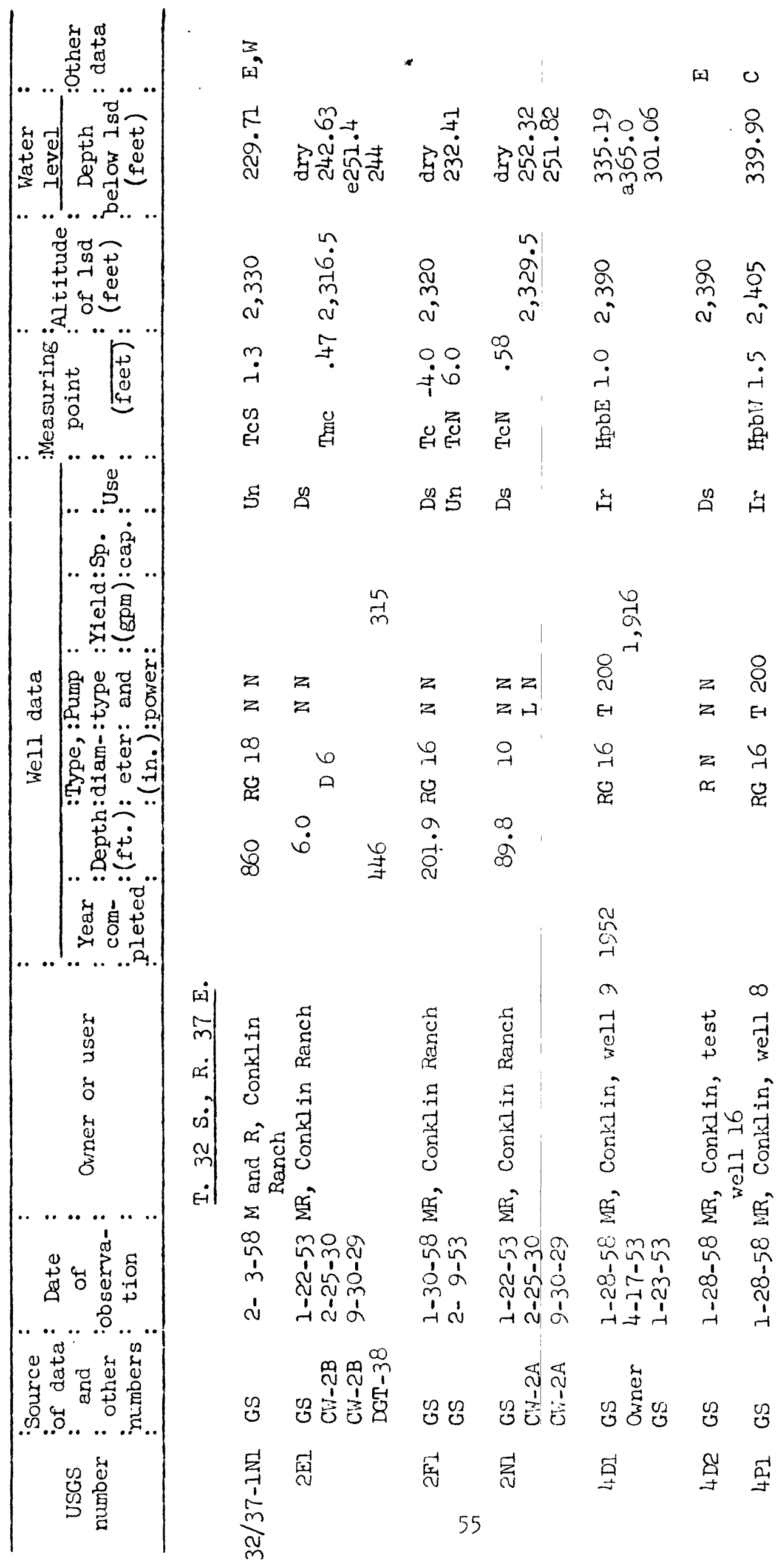




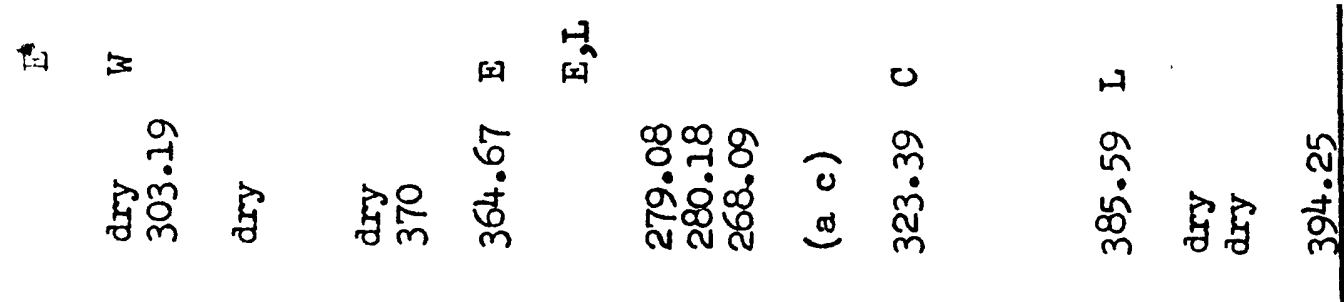

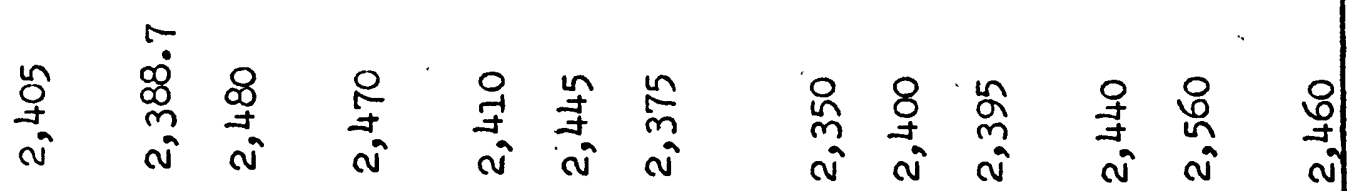

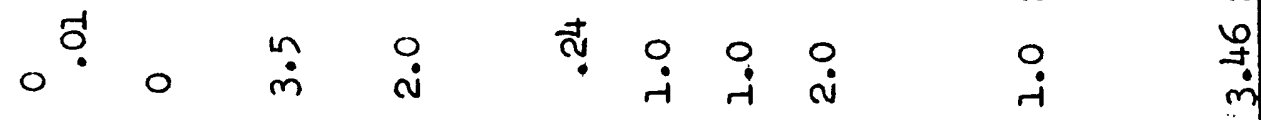

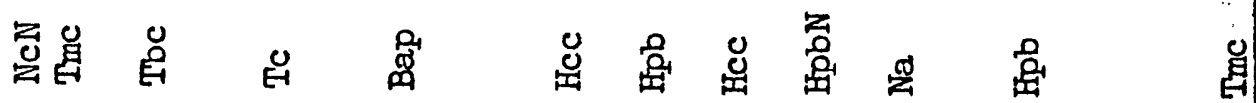

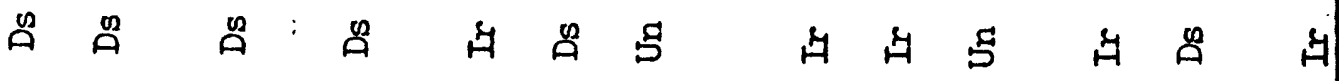

品 8

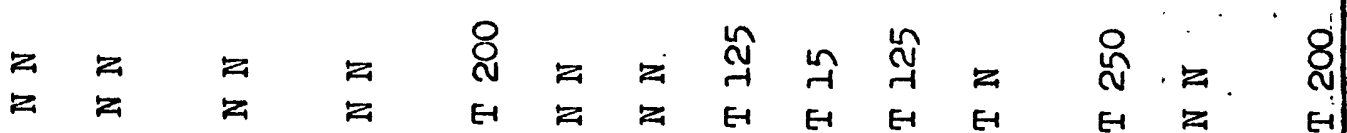

z

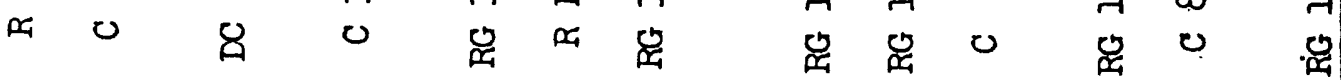

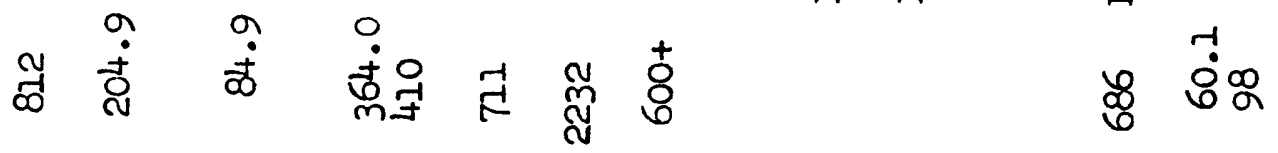

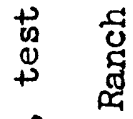

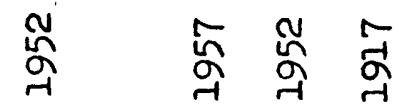

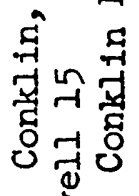

$\hat{\varepsilon}^{3}$ 출

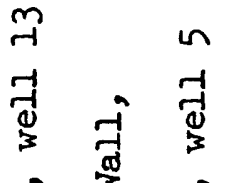

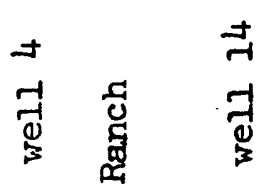

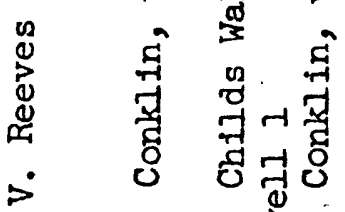

सं क्षे

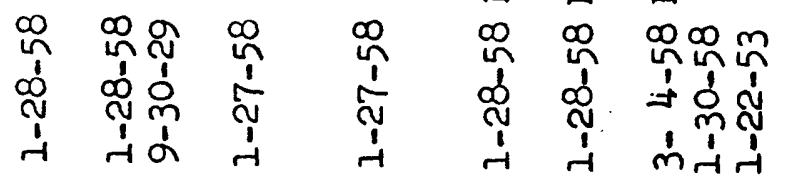

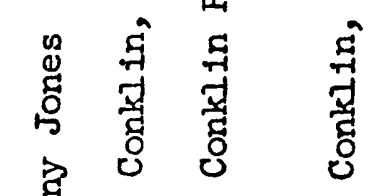

ผู่

ह

बै बे है

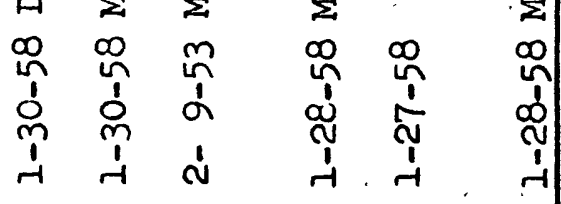

乌

边

它飠

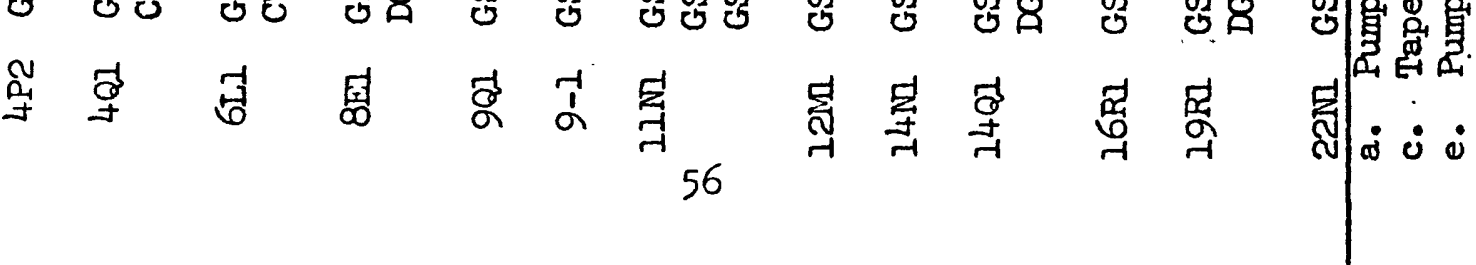




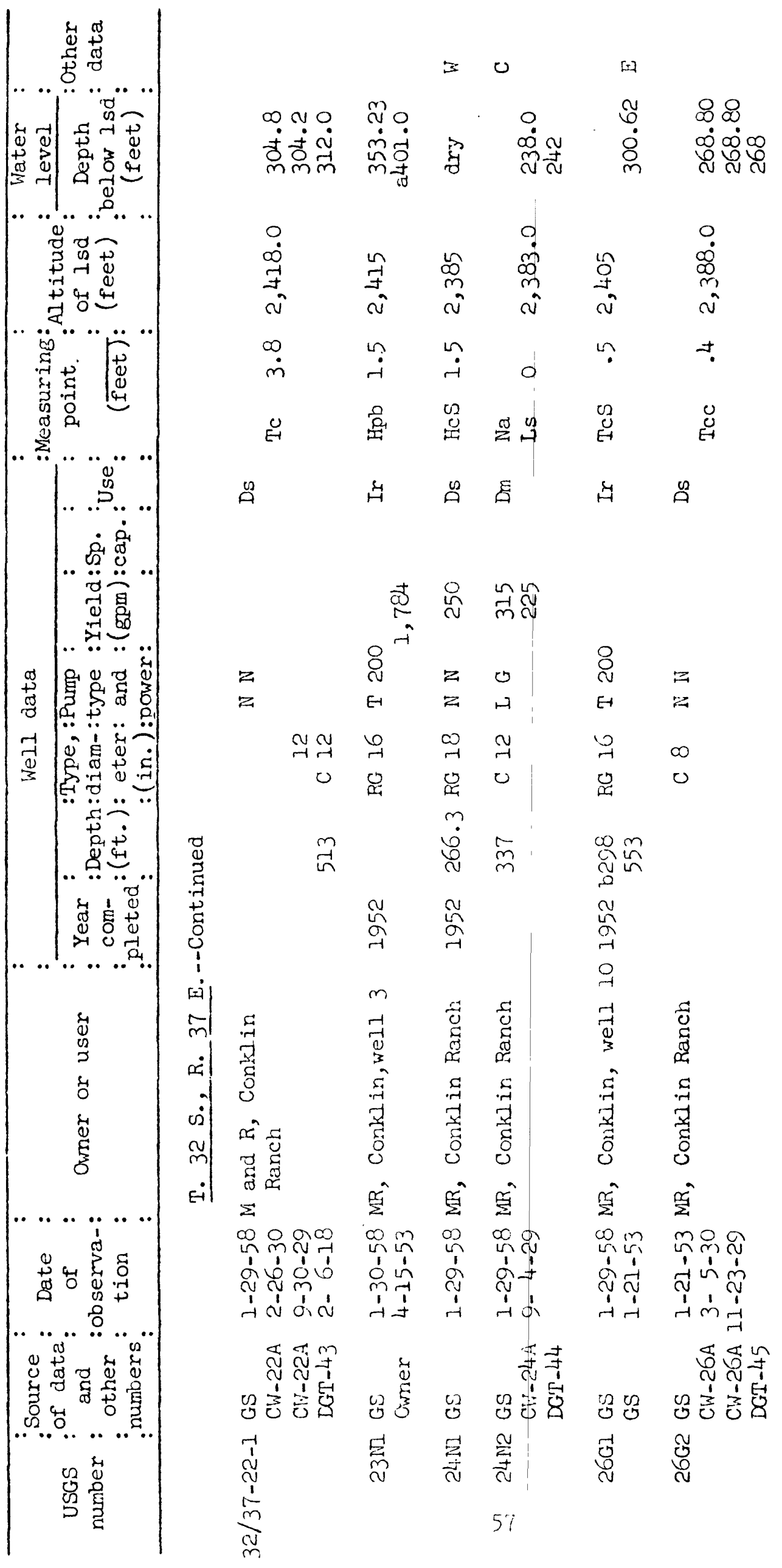




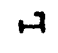

田回

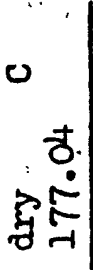

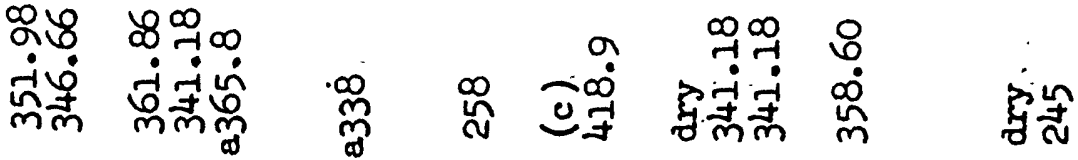

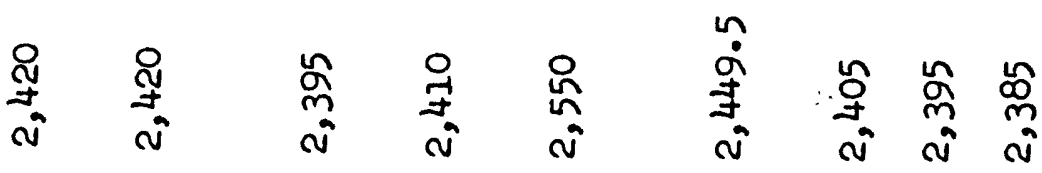

$\stackrel{-1}{-i}$

品 璦

-

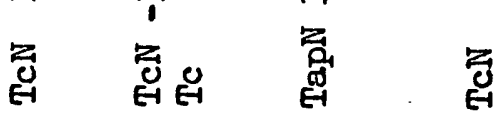

年

क्ष

\&

马 5 \& 8 \&

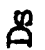

$8 \quad 8$ 윤

8 กั

-i

EH EH

$\begin{array}{lllll}-1 & & & \\ z & \ddots & z & z\end{array}$

穴

星 雚

r-1

EI Z Z

$\underset{-1}{9}$

$\begin{array}{llll}4 & z & \infty \\ 0 & 0 & 0 & 0\end{array}$

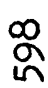

a

定

n

过

in 8 i $\frac{\dot{m}}{m}$

ช

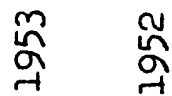

令

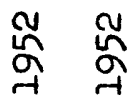

n $\rightarrow$ ब

-

न्न

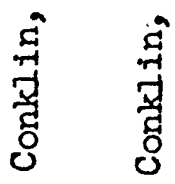

द્न म्न

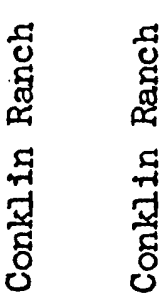

ज्ञ

息鬼.

岳

运

需

ผั่

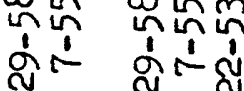

品角 品

ह่ं के

पं ते

\&ิ

ले

it

में

न

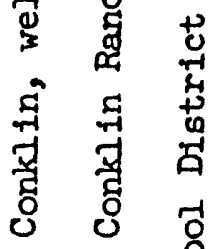

传

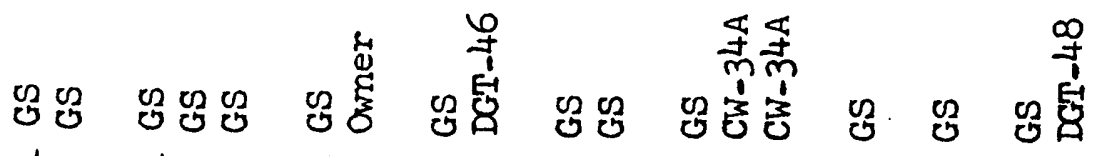

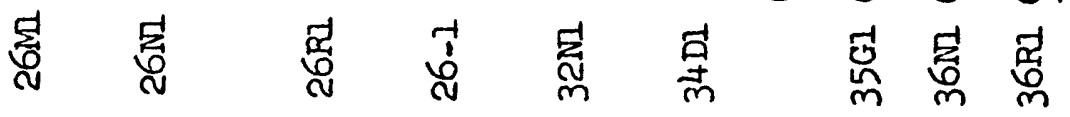




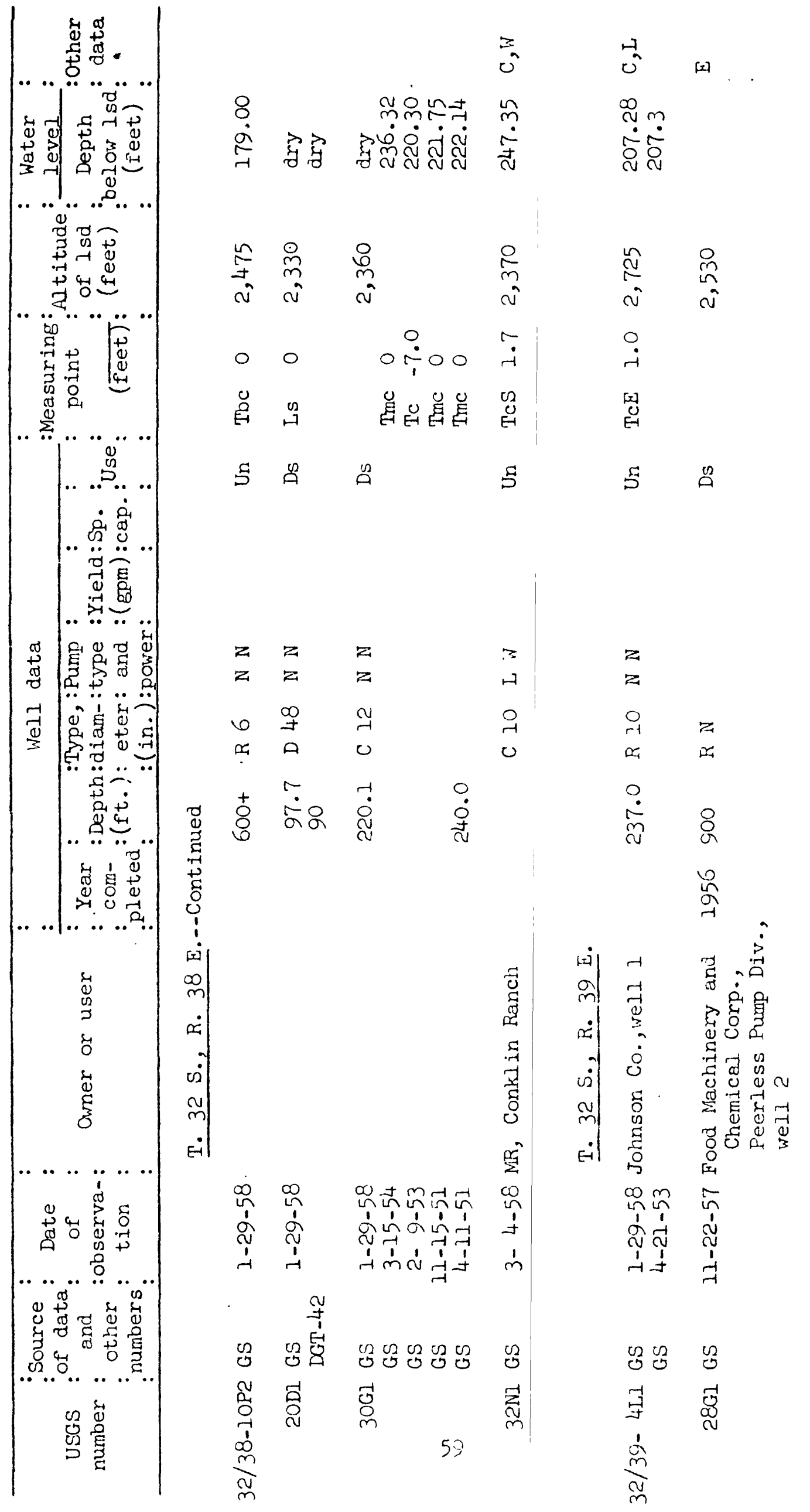




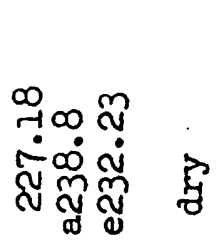

田

0

ar

宛

m

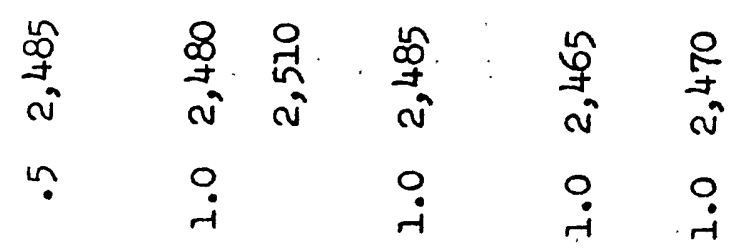

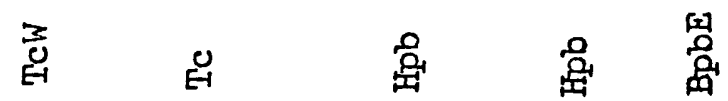

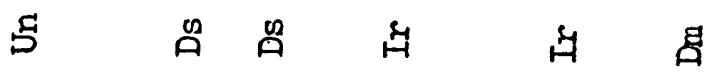

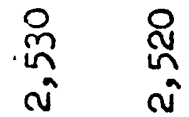

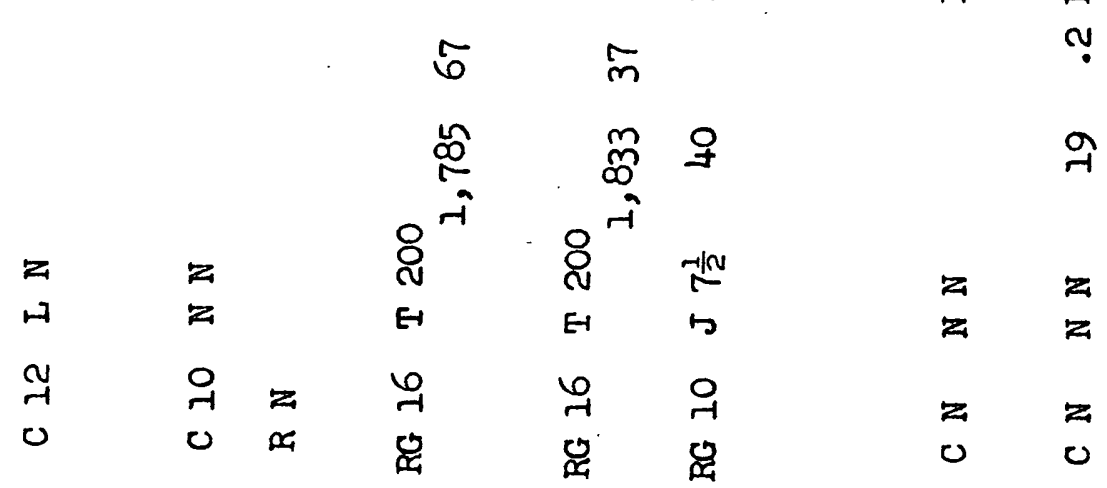

$\stackrel{\circ}{\stackrel{n}{N}} \quad \stackrel{\dot{m}}{N}$

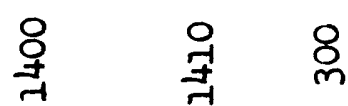

요 8

尔 号

志

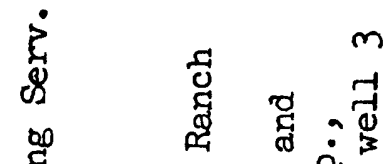

侌

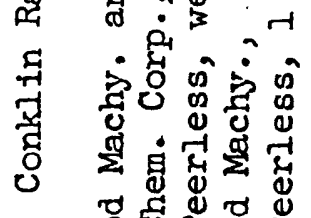

is

$\dot{0}$

$\infty$ 急

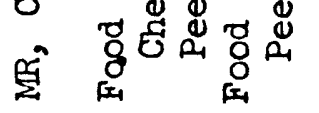

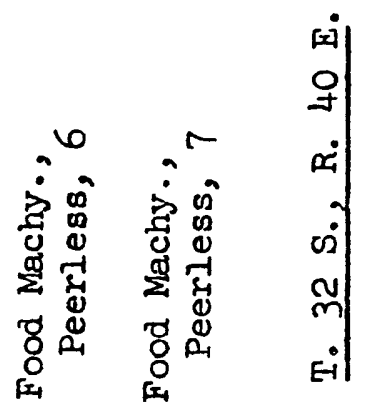

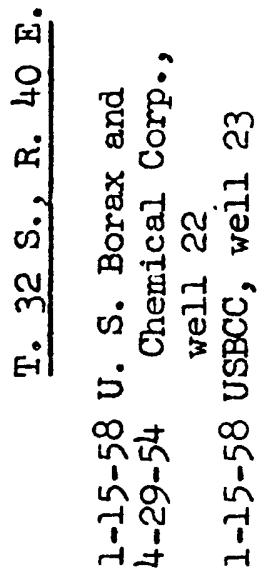

ह่ं ต่

帒

กิ กํํํํำ

-

ญิ่

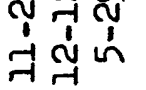

ญิ ญี

त्रें

î̀

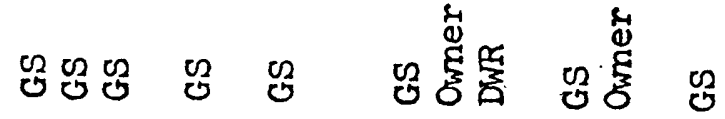

还

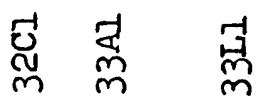

空 空

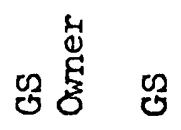

离 岳

ले

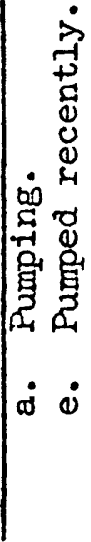


Table 2.- Cross index of other. well numbers and

U. S. Geological Survey numbers

The first column shows the number assigned to the well by others and the second column shows the Geological survey number assigned to the same well. The numbers in the first column are listed consecutively. Numbers missing in the consecutive listings are for wells outside the Fremont Valley and vicinity area or for wells for which no data are available.

Part 1. Nhumbers used by Thompson(1929)

\begin{tabular}{|c|c|c|c|c|c|}
\hline $\begin{array}{l}\text { Other: } \\
\text { number: }\end{array}$ & $\begin{array}{c}\text { USGS } \\
\text { number }\end{array}$ & $\begin{array}{l}\text { :Other: } \\
\text { :number: }\end{array}$ & $\begin{array}{c}\text { USGS } \\
\text { number }\end{array}$ & $\begin{array}{l}\text { :Other : } \\
\text { :number: }\end{array}$ & $\begin{array}{c}\text { USGS } \\
\text { number }\end{array}$ \\
\hline $3 A$ & $11 / 9-34 \mathrm{AI}$ & : 20 & $30 / 38-19 \mathrm{MI}$ & $: 36$ & $31 / 37-26 \mathrm{KI}$ \\
\hline $3 F$ & $29 / 39-12 \times 3$ & : $\quad 21$ & $19 \mathrm{KI}$ & 37 & $31 / 38-31 C 1$ \\
\hline 4 & $12 \times 2$ & $\begin{array}{l}: \quad 22 \\
:\end{array}$ & $30 \mathrm{EI}$ & $: 38$ & $32 / 37-2 E I$ \\
\hline 5 & $15 \mathrm{E} 1$ & $: \quad 2$ & 2OEI & $: \quad 39$ & $8 \mathrm{E} I$ \\
\hline 6 & $15 \mathrm{MI}$ & $:$ & $32 N 1$ & $: 40$ & I9RI \\
\hline 7 & $22 D 1$ & $24 \mathrm{~A}$ & $32-1$ & $: 41$ & 14Q1 \\
\hline 8 & 22D2 & 25 & $31 / 37-2-1$ & : 42 & $32 / 38-2001$ \\
\hline 9 & $22 E I$ & 26 & $10-1$ & 43 & $32 / 37-22-1$ \\
\hline 10 & $27 \mathrm{KI}$ & 27 & $10 Q 1$ & 44 & $24 \mathrm{N2}$ \\
\hline 11 & $33 \mathrm{Cl}$ & 28 & $12-1$ & $: 45$ & $26 \mathrm{G} 2$ \\
\hline 12 & $32 \pi$ & 29 & $13 \mathrm{BI}$ & $: 46$ & $26-1$ \\
\hline 13 & $30 / 38-8 G 1$ & 30 & $31 / 38-6 \mathrm{BI}$ & $: 48$ & $36 \mathrm{RI}$ \\
\hline 14 & $29 / 37-34 \mathrm{~B} 2$ & $\begin{array}{l}: \\
:\end{array}$ & $30 / 38-29-1$ & $: \quad 52$ & $12 / 10-31-1$ \\
\hline 15 & $30 / 38-30 \mathrm{OB} 2$ & $3 \geq A$ & $30 R I$ & $: \quad 54$ & $34 D 1$ \\
\hline 16 & $30 / 37-23 \mathrm{~J} 3$ & $\begin{array}{l}: \quad 32 \\
:\end{array}$ & $31 / 38-1.8 \mathrm{PI}$ & $: 55$ & $35 \mathrm{PI}$ \\
\hline 17 & $26-1$ & : $\quad 33$ & $31 / 37-30 F I$ & $: 56$ & $11 / 9-6 P 1$ \\
\hline 18 & 2610 & $: \quad 34$ & $32-1$ & $: 57$ & IEJI \\
\hline 19 & $36 \mathrm{Gl}$ & $:$ & $32 A 1$ & $:$ & \\
\hline
\end{tabular}


Part 2. Numbers used by Cyril Williams, Jr., (1930)

\begin{tabular}{|c|c|c|c|c|c|c|}
\hline $\begin{array}{l}\text { Other } \\
\text { number }\end{array}$ & $:$ & $\begin{array}{c}\text { USGS } \\
\text { number }\end{array}$ & $:$ & $\begin{array}{r}\text { Other } \\
\text { number }\end{array}$ & 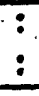 & $\begin{array}{l}\text { USGS } \\
\text { number }\end{array}$ \\
\hline $30 / 37-24-A$ & & $30 / 37-24 \mathrm{BI}$ & $:$ & $31 / 37-32-B$ & & $31 / 37-32 A 1$ \\
\hline $24-B$ & & $24 \mathrm{MI}$ & $\begin{array}{l}: \\
:\end{array}$ & $33-\mathrm{A}$ & & $33-1$ \\
\hline $24-C$ & & $24 R 2$ & : & $34-A$ & & $34 \mathrm{AI}$ \\
\hline $26-A$ & & $26-1$ & $:$ & $32 / 37-2-A$ & & $32 / 37-2 N$ \\
\hline $36-A$ & & $36 \mathrm{Gl}$ & $:$ & $2-B$ & & $2 \mathrm{FI}$ \\
\hline $30 / 38-30-A$ & & 30/38-30E1 & $:$ & $4-A$ & & 4QI \\
\hline $30-B$ & & $30 B 1$ & : & $6-A$ & & 6II \\
\hline $32-A$ & & $32 G 1$ & $:$ & 22-A & & $22-1$ \\
\hline $31 / 37-14-A$ & & $31 / 37-14 I I$ & : & $24-A$ & & $24 \mathrm{~N} 2$ \\
\hline $22-A$ & & 22QI & : & $26-A$ & & $26 \mathrm{G} 2$ \\
\hline $26-A$ & & $26 \mathrm{KI}$ & : & $34-A$ & & $34 \mathrm{DI}$ \\
\hline $30-A$ & & $30 F 1$ & $:$ & . & . & \\
\hline
\end{tabular}


Table 3.- Records of water levels in wells

Included are all known records of water-level measurements in wells where six or more measurements have been made; records for wells where less than six measurements have been made are shown in table 1 .

Altitudes given are in feet above mean sea level for the land-surface datum at the well. Land-surface datum is a plane of reference which approximates land surface. Altitudes given in whole feet are interpolated from topographic maps. Altitudes given in feet and tenths were determined by spirit leveling/from records by Cyril Williams, Jr. (1930)].

Measurements. All measurements of water level have been adjusted to depth below land-surface datum. That is, the altitudes of the measuring points as reported above land-surface datum have been subtracted from the water-level measurements below the described measuring point. Measurements at 14 wells ( $11 / 8-32 \mathrm{GI}, 11 / 9-17 \mathrm{NI}, 30 \mathrm{NI}, 34 \mathrm{AI}, 29 / 39-28 \mathrm{HI}, 30 / 37-24 \mathrm{MI}$, $30 / 38-32 \mathrm{EI}, 30 / 39-8 \mathrm{Al}, 31 / 37-8 \mathrm{Cl}, 13 \mathrm{BI}, 35 \mathrm{NI}, 32 / 37-1 \mathrm{NI}, 11 \mathrm{NI}$, and 32/38-32NI) are made by the Geological Survey during the spring and autumn each year. indicated.

All measurements are by the Geological Survey unless otherwise

11/8-20HL. D. W. Swanson. Depth 213.9 feet. Altitude about 2,380 feet. Measuring point: Top of casing 0.73 foot above 1 sd.

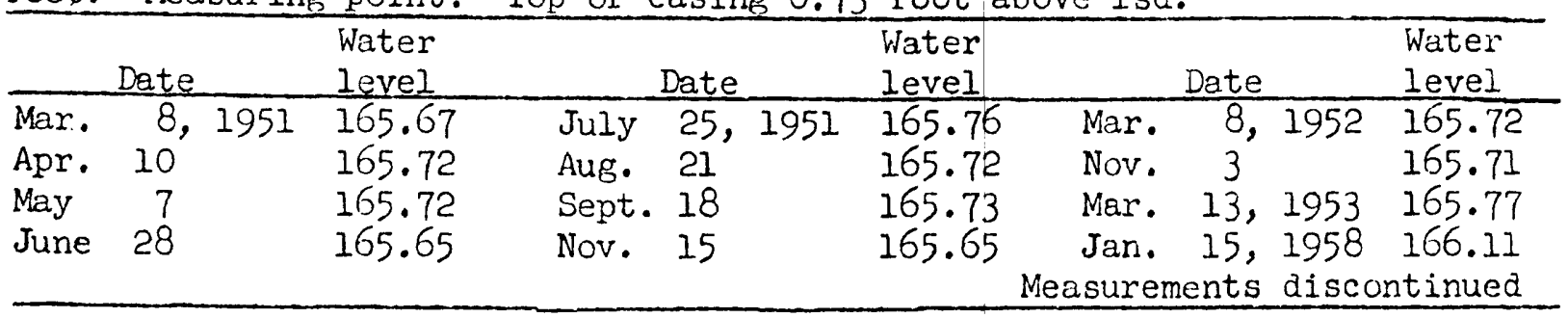

11/8-32Gl. Owner unknown. Depth 156.0 feet. Altitude about 2,340 feet. Weasuring point: Hole in casing cover 2.12 feet above 1 sa.

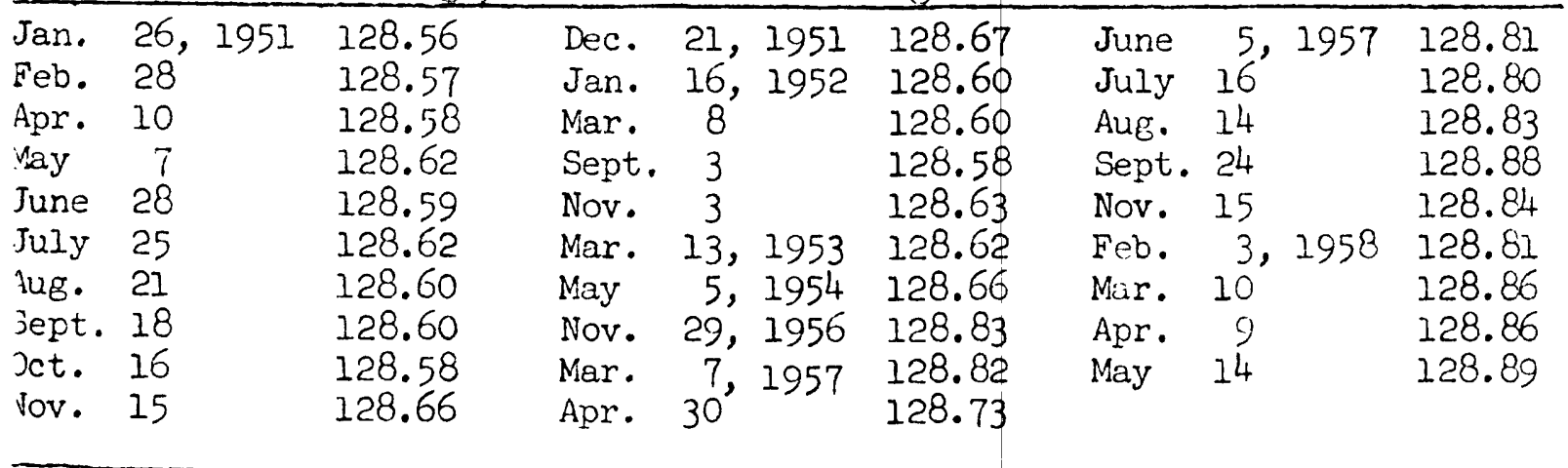


11/9-17NI(formerly 11/9-17Mn). Owner unknown. Depth 184.6 feet. Alt1tude about 2,324 feet. Measuring point: "Top of casing 0.5 foot above lsd.

\begin{tabular}{|c|c|c|c|c|c|c|}
\hline Date & $\begin{array}{l}\text { Water } \\
\text { level }\end{array}$ & & Date & $\begin{array}{l}\text { Water } \\
\text { level }\end{array}$ & $\therefore$ Date & $\begin{array}{l}\text { Water } \\
\text { level }\end{array}$ \\
\hline $\begin{array}{l}\text { Apr. 11, } 1951 \\
\text { Nov. 15 } \\
\text { Mar. } 8,1952 \\
\text { Nov. 3 } \\
\text { Mar. } 13,1953\end{array}$ & $\begin{array}{l}129.56 \\
129.58 \\
129.58 \\
129.53 \\
129.60\end{array}$ & $\begin{array}{l}\text { Apr. } \\
\text { May } \\
\text { Mar. } \\
\text { Nov. } \\
\text { Mar. }\end{array}$ & $\begin{array}{r}16,1953 \\
5,1954 \\
2,1955 \\
14 \\
22,1956\end{array}$ & $\begin{array}{l}129.55 \\
129: 67 \\
129: 82 \\
130: 76 \\
130.05\end{array}$ & $\begin{array}{l}\text { Nov. 27, } 1956 \\
\text { Mar: 6, } 1957 \\
\text { Nov: 19 } \\
\text { Mar: } 4,1958\end{array}$ & $\begin{array}{l}130.21 \\
129.70 \\
130.58 \\
130.67\end{array}$ \\
\hline
\end{tabular}

11/9-22Q1. J. C. Schecter. Depth 193 feet. Altitude about 2,320 feet. Measuring point: Top of wooden block over casing 1.0 foot abové I'sd.

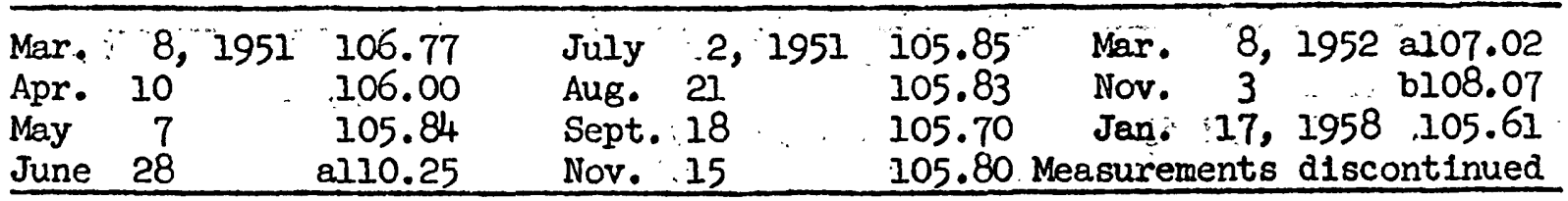

1I/9-30NI (formerly 1I/9-3IDI). Flourr. Depth 200 feet.' AltItude about 2,328 feet. Measuring point: Top of casing at 1sd.

\begin{tabular}{lrrrrrrrr}
\hline Jan. 26,1951 & 114.14 & Aug. 21, 1951 al14.61 & Mar. & 8, 1952 & 115.59 \\
Feb. 28 & 114.14 & Sept. 18 & 114.27 & July 27,1956 & 115.40 \\
Apr. 10 & 114.16 & Oct. 16 & al17.21 & Mar. 7,1957 & 115.60 \\
May & 7 & 114.18 & Nov. 16 & 114.35 & Nov. 15 & 115.91 \\
June 28 & 114.19 & Dec. 21 & 114.28 & Mar. 4,1958 & 115.81 \\
July 25 & 114.34 & Jan. 16, 1952 al17.14 & & 10 & 115.74 \\
\hline
\end{tabular}

11/9-30Q1. A. F. Green. Depth 169.1 feet. Alt1tude about 2,320 feet. Measuring point: Top of casing 1.2 feet above lsd.

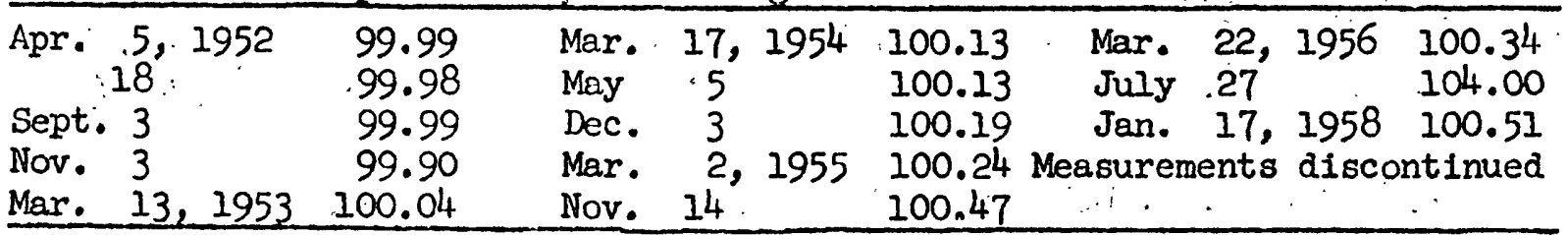

11/9-34AI (DGT, Antelope Valley 3). F. J. Schultz. Depth 193.5 feet. Altitude about 2,303 feet. Measuring point: Top of casing, south side, 0.4 foot above 1 sd.

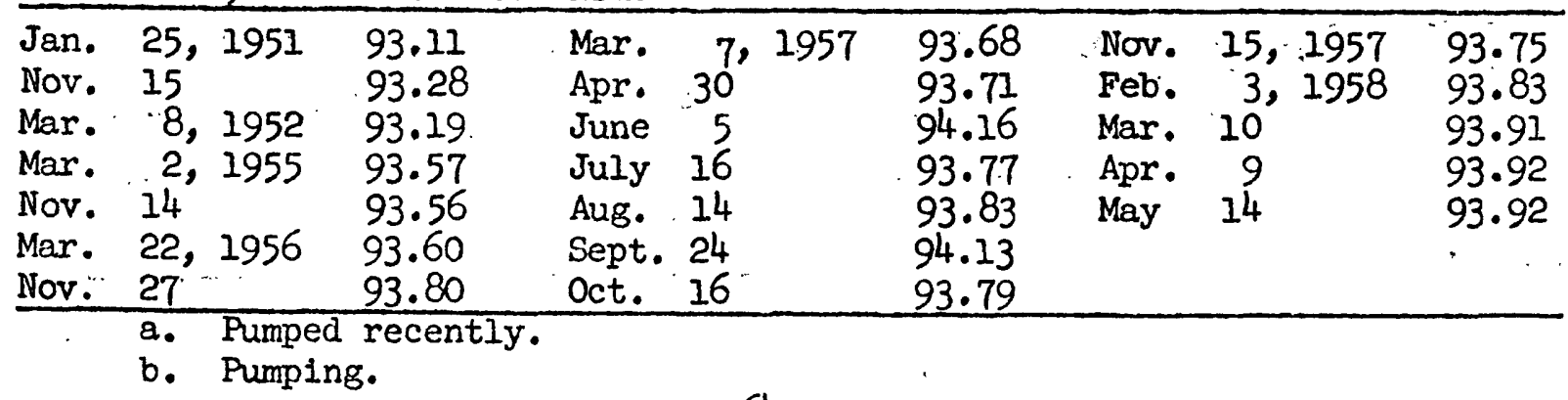


11/9-34KI. Millhollin. Depth 147 feet. Altitude about 2,300 feet. No access into well after July $27,1956$.

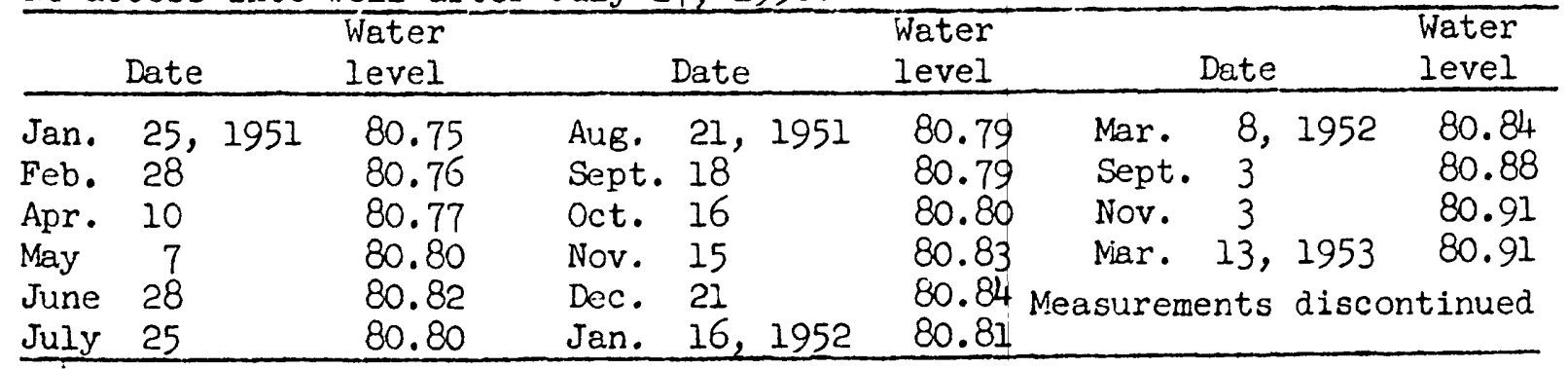

12/10-35PI (DGT-55). Owner unknown. Depth 271.8 feet prior to December 3, 1954, 194.I feet after that date. Altitude about 2,365 feet. Measuring point: Top of casing 1.0 foot above lsd.

\begin{tabular}{llllllll}
\hline Feb. 5 or & & Mar. 8, 1952 & 193.47 & Apr. & 21,1953 & 195.47 \\
6,1918 a cl99 & Sept. 10 & 193.50 & Dec. & 3,1954 dry \\
Apr. 11,1951 & 193.53 & Feb. 9, 1953 & 193.60 & & & \\
Nov. 15 & 193.48 & Apr. 16 & 197.3 & & \\
\hline
\end{tabular}

29/39-28Hl. Owner unknown. Depth 205.7 feet. Altitude about 2,100 feet. Measuring point: Top of casing 2.0 feet above Isd.

\begin{tabular}{lcclclllll}
\hline Apr. & 30,1953 & 173.09 & Nov. & 15,1955 & 173.82 & Nov. 22,1957 & 174.59 \\
Mar. & 17,1954 & 173.23 & Mar. & 20,1956 & 173.93 & Feb. & 13,1958 & 174.50 \\
Dec. & 3 & & 173.50 & Nov. 28 & & 174.16 & & & \\
Mar. & 2,1955 & 173.57 & Mar. & 6,1957 & 174.28 & & & \\
\hline
\end{tabular}

30/37-24MI (formerly 30/37-24NI). Owner unknown. Depth unknown. Altitude 2,000.0 feet. Measuring point: Top of casing at lsd.

\begin{tabular}{|c|c|c|c|c|c|c|c|c|c|c|c|}
\hline $\begin{array}{l}\text { Oct. } \\
\text { Mar. } \\
\text { Mar. } \\
\text { Nov. }\end{array}$ & $\begin{array}{r}3, \\
18, \\
17 \\
30\end{array}$ & $\begin{array}{l}1929 \\
1953 \\
1954\end{array}$ & $\begin{array}{r}\mathrm{d} 37.50 \\
32.64 \\
39.21 \\
41.15\end{array}$ & $\begin{array}{l}\text { Mar. } \\
\text { Nov. } \\
\text { Mar. } \\
\text { Aug. }\end{array}$ & $\begin{array}{l}2, \\
15 \\
19 \\
29\end{array}$ & $\begin{array}{l}1955 \\
1956\end{array}$ & $\begin{array}{l}40.85 \\
41.36 \\
40.90 \\
41.64\end{array}$ & $\begin{array}{l}\text { Nov. } \\
\text { Mar. } \\
\text { Nov. } \\
\text { Feb. }\end{array}$ & $\begin{array}{r}27 \\
6, \\
22 \\
26\end{array}$ & $\begin{array}{l}1956 \\
1957\end{array}$ & $\begin{array}{l}41.95 \\
43.84 \\
43.77\end{array}$ \\
\hline
\end{tabular}

30/38-8N1. E. S. MicKendry. Depth 52 feet. Altitude about 1,950 feet. Measuring point: Top of casing at Isd.

\begin{tabular}{rrrrrrrrr} 
May & 5,1953 & 22.89 & Nov. & 30,1954 & 22.23 & Oct. & 10,1956 & 23.42 \\
Mar. 17,1954 & 21.90 & Mar. & 2,1955 & 22.50 & Feb. & 14,1958 & 25.45 \\
\hline
\end{tabular}

30/38-32El. M and $R$, Cantil Ranch. Depth 107.4 feet. Altitude about 1,980 feet. Measuring point: Top of casing 1.0 foot above 1 sd.

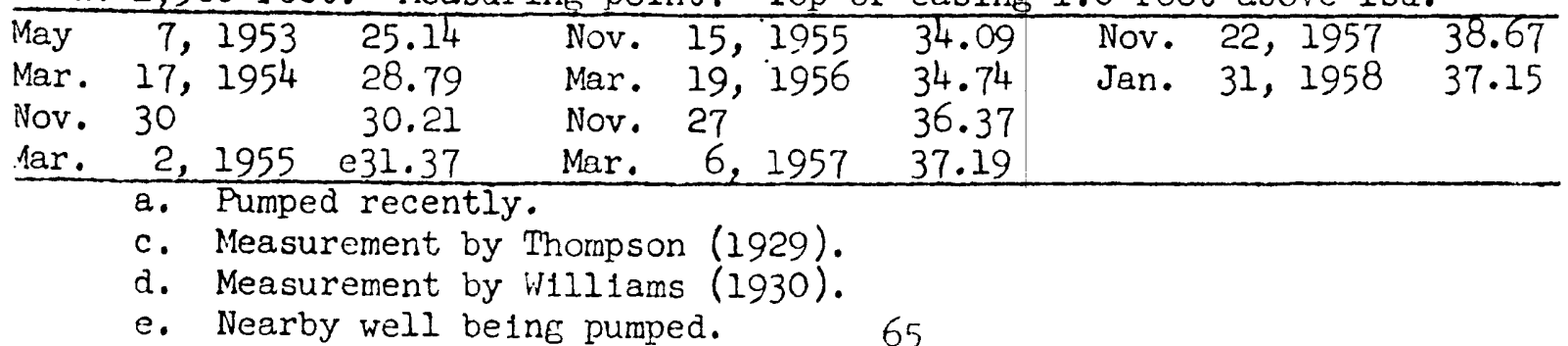


30/39-8Al (formerly 30/39-5RI). Owner unknown. Depth 268.3 feet. Altitude about 2,075 feet. Measuring point: Top of casing 1.0 foot above lsd.

\begin{tabular}{|c|c|c|c|c|c|c|c|c|}
\hline & Date & $\begin{array}{l}\text { Water } \\
\text { level }\end{array}$ & . & Date & $=$ & $\begin{array}{l}\text { Water } \\
\text { level }\end{array}$ & Date & $\begin{array}{l}\text { Water } \\
\text { level } \\
\end{array}$ \\
\hline $\begin{array}{l}\text { Apr. } \\
\text { Mar. } \\
\text { Dec. } \\
\text { Mar. }\end{array}$ & $\begin{array}{c}30,1953 \\
17,1954 \\
3 \\
2,1955\end{array}$ & $\begin{array}{l}136.64 \\
136.89 \\
136.70 \\
136.55\end{array}$ & $\begin{array}{l}\text { Nov. } \\
\text { Mar. } \\
\text { Oct. } \\
\text { Nov. }\end{array}$ & $\begin{array}{l}15, \\
20 \\
11 \\
28\end{array}$ & $\begin{array}{l}1955 \\
1956\end{array}$ & $\begin{array}{l}136.74 \\
136.83 \\
138.01 \\
138.15\end{array}$ & $\begin{array}{ll}\text { Mar. } & 6,1957 \\
\text { Nov. } 22 & \\
\text { Feb. } & 12,1958\end{array}$ & $\begin{array}{l}138.13 \\
138.40 \\
137.91\end{array}$ \\
\hline
\end{tabular}

31/37-8Cl. $M$ and $R$, Conklin Ranch, well 16. Depth 650 feet. Altitude about 2,190 feet. Measuring point: Edge of pump base; southwest side, 1.95 feet above 1 sd and 0.95 foot above top of casing.

\begin{tabular}{|c|c|c|c|c|c|c|c|c|c|c|c|}
\hline $\begin{array}{l}\text { Mar. } \\
\text { Nov. } \\
\text { Mar. }\end{array}$ & $\begin{array}{l}17 \\
30 \\
2\end{array}$ & $\begin{array}{l}1954 \\
1955\end{array}$ & $\begin{array}{l}149.30 \\
170.66 \\
170.70\end{array}$ & $\begin{array}{l}\text { Nov: } \\
\text { Mar: } \\
\text { Nov. }\end{array}$ & $\begin{array}{l}15, \\
19, \\
28\end{array}$ & $\begin{array}{l}1955 \\
1956\end{array}$ & $\begin{array}{l}171.49 \\
171.81 \\
173.04\end{array}$ & $\begin{array}{l}\text { Mar. } \\
\text { Nov. } \\
\text { Mar. }\end{array}$ & $\begin{array}{r}6, \\
20, \\
4,\end{array}$ & $\begin{array}{l}1957 \\
1958 \\
195\end{array}$ & $\begin{array}{l}174.68 \\
174.14 \\
174.30 \\
\end{array}$ \\
\hline
\end{tabular}

31/37-13B1 (DGT-29). Lew1s Ryan. Depth 400 feet. Altitude about 2,140 feet. Measuring point: Top of casing 1.0 foot above 1 sd.

\begin{tabular}{|c|c|c|c|c|c|c|c|c|c|c|}
\hline $\begin{array}{l}\text { Jan. } \\
\text { Mar. } \\
\text { Dec. }\end{array}$ & $\begin{array}{c}22, \\
15, \\
3\end{array}$ & $\begin{array}{l}1917 \\
1953 \\
1954\end{array}$ & $\begin{array}{l}c 130 \\
155.84 \\
163.60 \\
167.74\end{array}$ & $\begin{array}{l}\text { Mar. } \\
\text { Nov. } \\
\text { Mar. } \\
\text { Aug. }\end{array}$ & $\begin{array}{l}2, \\
15 \\
22, \\
17\end{array}$ & $\begin{array}{l}1955 \\
1956\end{array}$ & $\begin{array}{l}167.13 \\
169.78 \\
170.30 \\
172.75\end{array}$ & $\begin{array}{l}\text { Nov. } \\
\text { Mar. } \\
\text { Mar. }\end{array}$ & $\begin{array}{r}27,1956 \\
6,1957 \\
4,1958\end{array}$ & $\begin{array}{l}173.41 \\
174.28 \\
177.80\end{array}$ \\
\hline
\end{tabular}

31/37-22Ql. I. W. Giddings. Depth 500 feet. Altitude 2,260.0 feet. Measuring point: Top of casing at Isd.

\begin{tabular}{lllllllll}
\hline Sept. 30, 1929 & d253.0 & Mar. 2,1955 & 262.93 & Mar. & 6,1957 & 268.97 \\
Jan. 22, 1953 & 257.77 & Nov. 15 & 265.09 & Nov. 22 & & 269.32 \\
Mar. 15, 1954 & 260.15 & Mar. 22,1956 & 265.99 & & & \\
Dec. $\quad 3$ & 262.14 & Nov. 27 & 267.91 & & & \\
\hline
\end{tabular}

32/37-1NI. M and R, Conklin Ranch. Depth 860 feet. Altitude about 2,330 feet. Measuring point: Top of casing 1.3 feet above Isd on February 3, 1958, and 9.0 feet above isd before that date.

\begin{tabular}{lcccccccc}
\hline Feb. & 9,1953 & 223.59 & Nov. & 15,1955 & 225.43 & Nov. & 22,1957 & 230.11 \\
Mar. & 15,1954 & 226.48 & Mar. & 22,1956 & 225.28 & Feb. & 3,1958 & 229.71 \\
Dec. & 3 & 226.03 & Nov. 27 & & 227.72 & & & \\
Mar. & 2,1955 & 225.16 & Mar. & 6,1957 & 229.98 & & & \\
\hline
\end{tabular}

32/37-4Q1. $M$ and R, Conklin Ranch (formerly R. D. Floyd). Depth unknown prior to March 6, 1957, 204.9 feet thereafter. Altitude 2,388.7 feet. Measuring point: Top of casing at lsd.

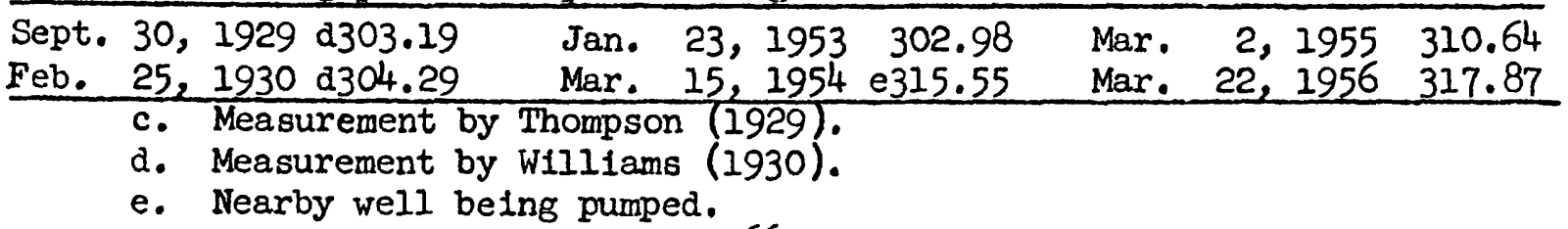


32/37-24NI. $M$ and $R$, Conklin Ranch. Depth about 480 feet prior to March 6, 1957, and 266.3 feet thereafter. Altitude about 2,385 feet.

Measuring point: Hole in side of casing 1.5 feet above Isd.

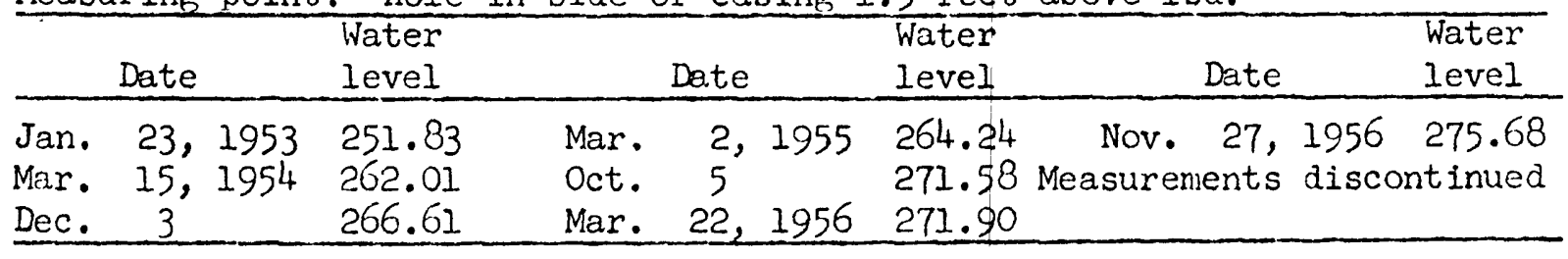

32/38-32NI. M and $R$, Conklin Ranch. Depth unknown. Altitude about 2,370 feet. Measuring point: Top of casing 1.7 feet above 1 sd.

\begin{tabular}{|c|c|c|c|c|c|c|c|c|c|c|c|}
\hline $\begin{array}{l}\text { Apr. } \\
\text { Nov. } \\
\text { Mar. } \\
\text { Jan. } \\
\text { Feb. }\end{array}$ & $\begin{array}{c}11, \\
15 \\
8, \\
22, \\
9\end{array}$ & $\begin{array}{l}1951 \\
1952 \\
1953\end{array}$ & $\begin{array}{l}223.35 \\
223.20 \\
223.82 \\
224.08 \\
224.16\end{array}$ & $\begin{array}{l}\text { Apr. } \\
\text { Mar. } \\
\text { Dec. } \\
\text { Mar. } \\
\text { Oct. }\end{array}$ & $\begin{array}{l}16, \\
15, \\
3 \\
2, \\
7\end{array}$ & $\begin{array}{l}1953 \\
1954\end{array}$ & $\begin{array}{l}225.30 \\
231.79 \\
235.90 \\
236.03 \\
237.56\end{array}$ & $\begin{array}{l}\text { Mar. } \\
\text { Nov. } \\
\text { Mar. } \\
\text { Nov. } \\
\text { Mar. }\end{array}$ & $\begin{array}{c}22, \\
27 \\
6, \\
19 \\
4,\end{array}$ & $\begin{array}{l}1957 \\
1958\end{array}$ & $\begin{array}{l}239.14 \\
242.36 \\
243.80 \\
246.62 \\
247.35\end{array}$ \\
\hline
\end{tabular}


Table 4.- Drillers' logs of wells

10/7-6B2. Boron Community Services District, well 6. Alt1tude about 2,460 feet. Drilled by Barber-Bridge Drilling Corp. in June

1955. 12-inch casing, zero to 454 feet, perforated from 353 to 363 feet.

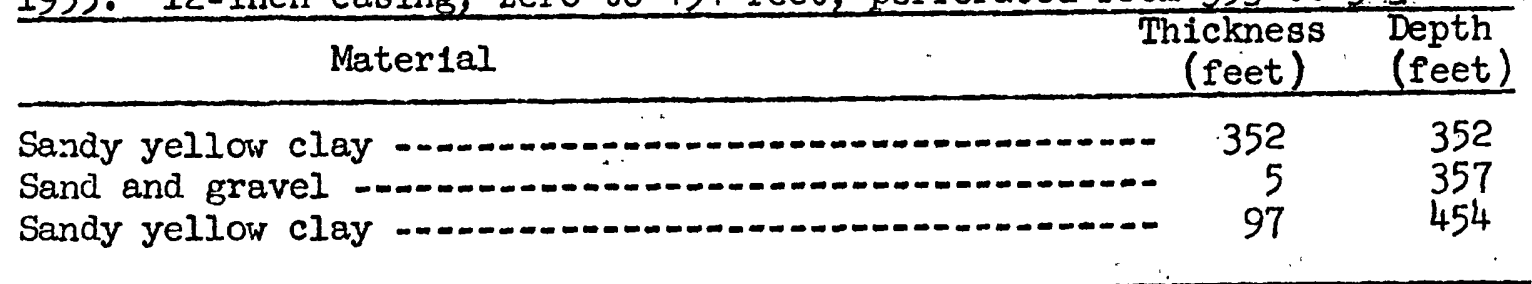

11/7-32E]. Boron Community Services District, well 8. Altitude about 2,455 feet. Drilled by Rottman Drilling Co. in July 1956. 10-1nch casing zero to 502 feet, perforated 262 to 502 feet.

\begin{tabular}{|c|c|c|}
\hline \multicolumn{3}{|l|}{ Clay and sand } \\
\hline Clay and gravel & 22 & 72 \\
\hline Clay and sand & 22 & 94 \\
\hline Coarse sand & 22 & 116 \\
\hline Coarse sand and clay .... & 24 & 140 \\
\hline 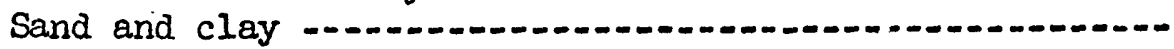 & 44 & 184 \\
\hline Sand and clay streaks ... & 26 & 210 \\
\hline 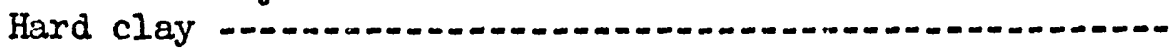 & 22 & 232 \\
\hline Clay and gravel ............. & 24 & 256 \\
\hline Clay and sand streaks & 22 & 278 \\
\hline Gravel and clay streaks ...- & 22 & 300 \\
\hline Gravel & 23 & 323 \\
\hline Boulders, clay, and sand & 22 & 345 \\
\hline Gravel and boulders & 26 & 37. \\
\hline pack gravel & 21 & 39 \\
\hline pack clay and gravel & 23 & 41 \\
\hline clay and gravel ... & 66 & 48 \\
\hline el and hard clay ... & 21 & 502 \\
\hline
\end{tabular}

11/7-32G2. Franklin. Altitude about 2,460 feet. Drilled by orner in 1954. 5-inch casing. (Log not complete.)

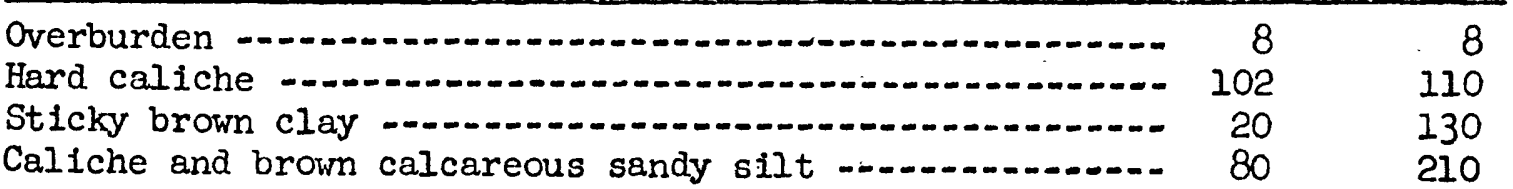


11/7-32HI. Boron Development Co. Altitude about 2,465 feet. Drilled by Rottman Drilling Co. In April 1956. 8-inch casing zero to 429 feet, perforated from 253 to 429 feet.

\begin{tabular}{|c|c|c|}
\hline Material & $\begin{array}{c}\text { Thickness } \\
\text { (feet) }\end{array}$ & $\begin{array}{l}\text { Depth } \\
\text { (feet) }\end{array}$ \\
\hline Sand and clay & 25 & 25 \\
\hline Sand & 25 & 50 \\
\hline Coarse sand & 30 & 80 \\
\hline Clay and coarse sand & 12 & 92 \\
\hline Sand and clay streaks & 30 & 122 \\
\hline Coarse sand and clay & 24 & 146 \\
\hline Coarse sand and rocks & 13 & 159 \\
\hline Sand and clay streaks & 23 & 182 \\
\hline Sandy clay & 23 & 205 \\
\hline Coarse sand and clay & 45 & 250 \\
\hline Clay and coarse sand & 70 & 320 \\
\hline Coarse sand & 20 & 340 \\
\hline Coarse sand, clay streaks & 37 & 377 \\
\hline Boulders and coarse sand & 18 & 395 \\
\hline Coarse sand and clay streaks & 10 & 405 \\
\hline Coarse gravel & 24 & 429 \\
\hline
\end{tabular}

11/7-32KI. Boron Community Services District, well 10. Altitude about 2,460 feet. Drilled by Rottman Drilling Co. in July 1957. 10-1nch casing zero to 600 feet, uncased hole 600 to 684 feet, perforated from 400 to 600 feet.

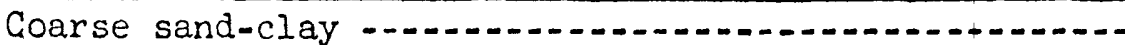

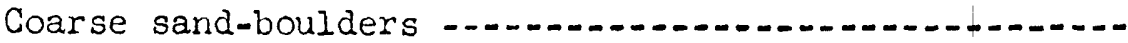

Boulders-sand -.......

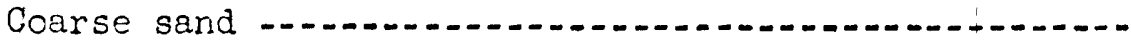

Cement sand

Hard cement sand -...

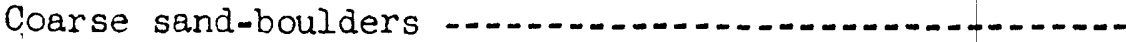

Boulders-fine sand

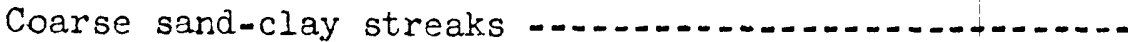

Sand and clay

Coarse sand and clay -........

Clay-coarse sand -...............

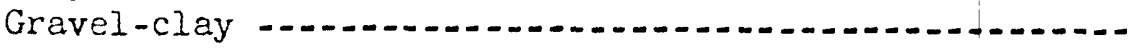

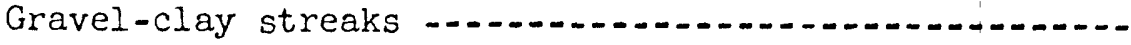

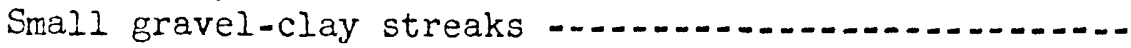

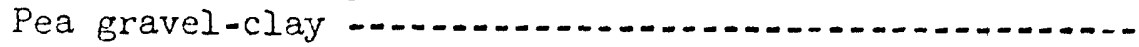

Coarse gravel-clay -.......

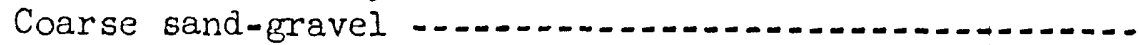

Coarse gravel-boulders .....

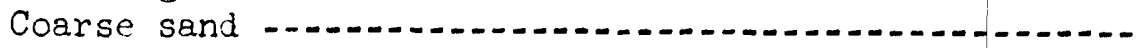

Coarse sand-clay streaks -

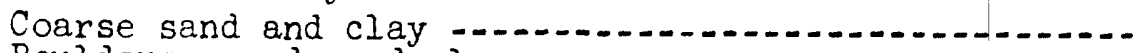

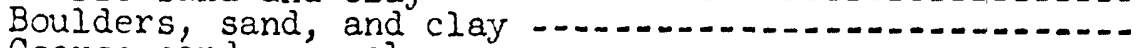

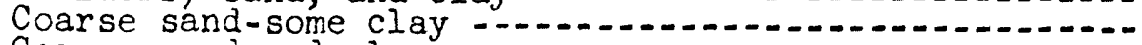

Coarse sand and clay

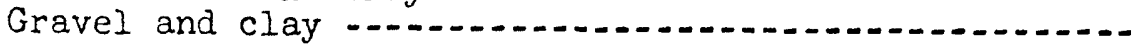


11/7-32K-.--Continued.

\begin{tabular}{|c|c|c|}
\hline Material & $\begin{array}{l}\text { Thickness } \\
\text { (feet) }\end{array}$ & $\begin{array}{l}\text { Depth } \\
\text { (feet) }\end{array}$ \\
\hline $\begin{array}{l}\text { Boulders-clay } \\
\text { Gravel and clay } \\
\text { Coarse sand-small boulders } \\
\text { Clay and coarse sand } \\
\text { Boulders, coarse sand }\end{array}$ & $\begin{array}{r}22 \\
23 \\
53 \\
7 \\
1\end{array}$ & $\begin{array}{l}600 \\
623 \\
676 \\
683 \\
684\end{array}$ \\
\hline
\end{tabular}

11/7-32м2. Boron Community Services District, well 9. Altitude about 2,450 feet. Drilled by. Rottman Drilling Co. in November 1956. 10-inch casing zero to 522 feet, perforated from 287 to 522 feet.

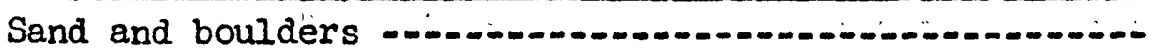

Sand, clay, and boulders -.......................................

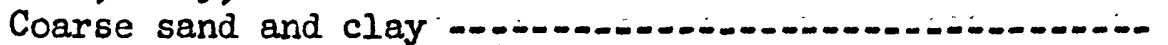

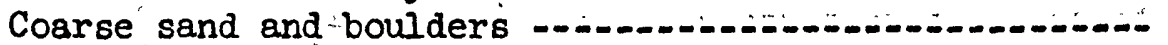

Coarse sand, some clay -.........

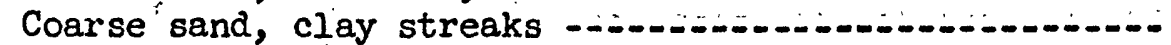

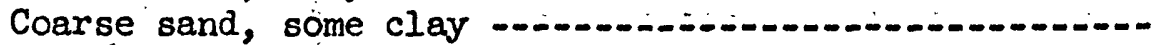

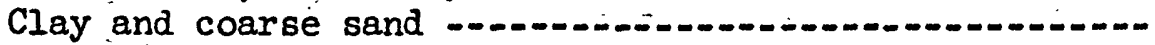

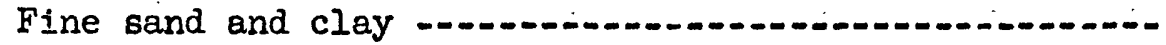

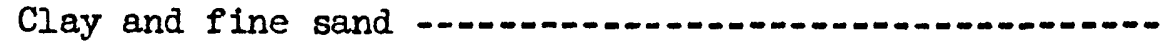

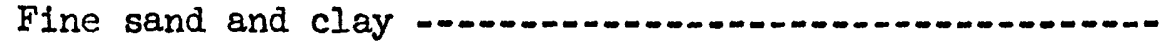

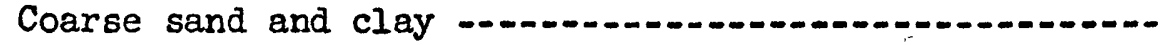

Boulders and coarse sand .................................

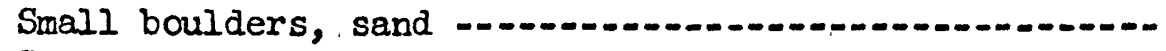

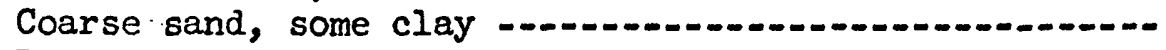

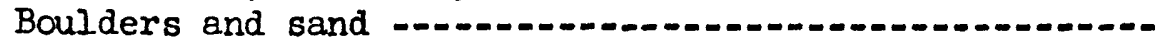

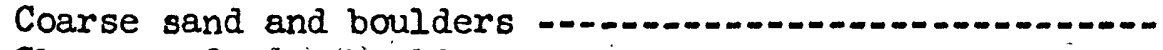

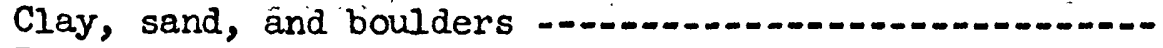

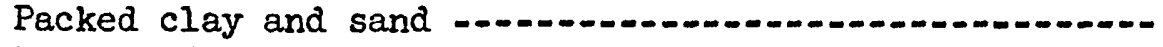

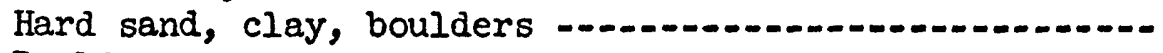

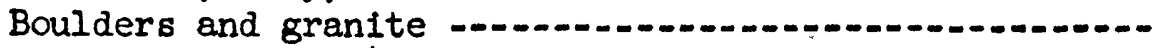

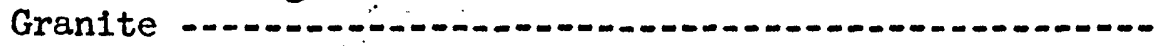

25

15

20

20

25

22

23

44

22

22

22

78

24

10

28

8

22

22

22

22

10

$24 \quad 530$


11/8-2NI. U. S. Borax and Chemical Corp., well 2. Altitude about 2,480 feet. Drilled by owner in 1936. 14-inch casing.

\begin{tabular}{|c|c|c|}
\hline Material & $\begin{array}{l}\text { hickness } \\
\text { (feet) }\end{array}$ & $\begin{array}{l}\text { Depth } \\
\text { (feet) }\end{array}$ \\
\hline Decomposed grantte & 95 & 95 \\
\hline Gray sandy clay & 75 & 170 \\
\hline Red sandy clay & 15 & 185 \\
\hline "Water" & 2 & 187 \\
\hline Red sandy clay & 18 & 205 \\
\hline "Water" & 2 & 207 \\
\hline Red sandy clay & 8 & 215 \\
\hline "Water" & 2 & 217 \\
\hline Red sandy clay & 38 & 255 \\
\hline Gravel water, large flow & 9 & 264 \\
\hline Hard shell & 4 & 268. \\
\hline Decomposed granite & 26 & 294 \\
\hline Hard shell & 2 & 206 \\
\hline Decomposed granite & 12 & 308 \\
\hline Hard shell & 3 & 311 \\
\hline Decomposed granite & 4 & 315 \\
\hline Very soft & 3 & 318 \\
\hline Decomposed granite & 9 & 327 \\
\hline Basalt, very hard & 9 & 336 \\
\hline
\end{tabular}

11/8-2P1. U. S. Borax and Chemical Corp., well 3. Altitude about 2,490 feet. Drilled by owner in 1941.

Sand, gravel, and clay ....................... 336

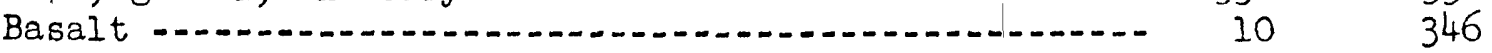

11/8-2-1. U. S. Borax and Chemicàl Corp., well 1. Altitude about 2,490 feet. Drilled by owner in 1926.

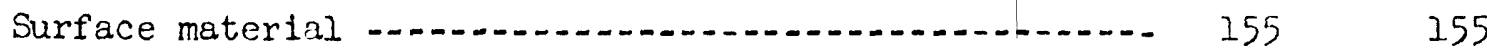

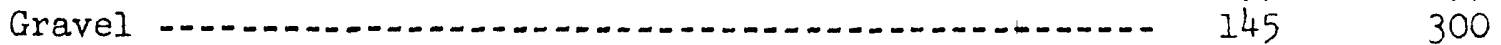

11/8-2-2. U. S. Borax and Chemical Corp., well 4. Altitude about 2,480 feet. Drilled by owner.

Sand, gravel, and clay _........ $270 \quad 270$

11/8-2-3. U. S. Borax and Chemical Corp., well 5. Altitude about 2,490 feet. Drilled by owner.

Surface sand and gravel ........................ 332

Basalt ............... $20 \quad 352$ 
11/8-2-4. U. S. Borax and Chemical Corp., well 6. Altitude about 2,480 feet. Drilled by owner.

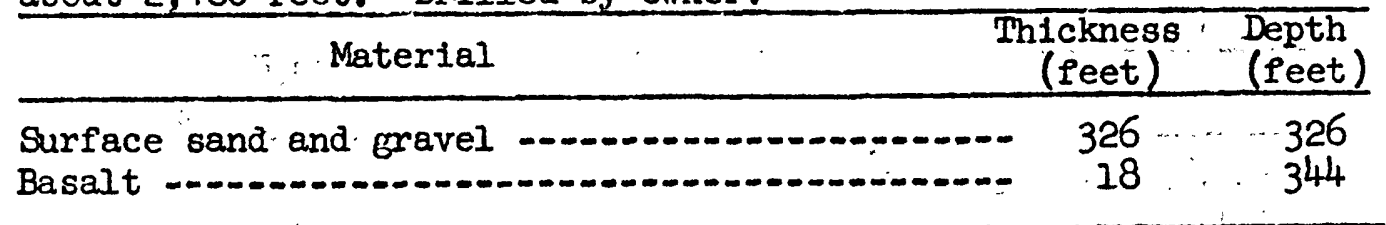

11/8-3Cl...U. S. Borax and Chemical Corp., well 25. Altitude about 2,490 feet. Drilled by owner in June 1954. 16-inch casing was removed after well was test pumped,

Sand, clay and gravel; sand, medium to finegrained, clay light brow, gravel pebble

to boulder size $40: \quad 40$

Gravel and clay; gravel coarse, clay

light brown .......... 1252

Sand, clay and gravel; sand medium-grain,

light brown clay and coarse gravel _........ $133 \quad 185$

Clay and sand; clay light yellowish brown, sand very fine to coarse, subangular ........

$60 \quad 245$

Clay; bright reddish-brown, plastic possibly some bentonite 250

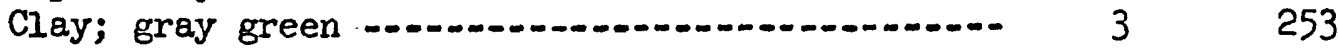

Clay; redd1sh-brown containing red decomposed inclusions which appear as volcanic origin -.- $12 \quad 265$

Clay; mixed red, green and yellow .............. 16

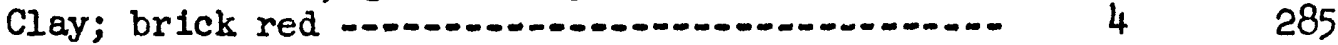

Clay; reddish nodules of clay and yellow decomposed clay inclusions. Some streaks of greenish sandstone 295

Clay; mixed red, yellow, brow and gray ....... $14 \quad 309$

Clay; yellow, hard ......................... 11

Clay; red and blue with streaks of gravel ...... 8328

Clay; redd1sh brown ............................ 345

Clay; gray with occasional streaks of reddish-brown sandy clay, some altered

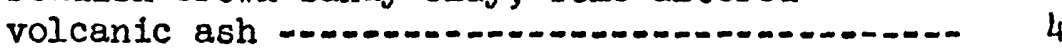

Clay; dark reddish-brown ...................... 1

Clay; gray-brown with streaks of gray, possibly altered volcantc ash ............. $40 \quad 440$ Sandstone; gray, fine grain, clay ............. 9449

11/8-3El. U. S. Borax and Chemical Corp., well 13. Altitude about 2,465 feet. Drilled by owner in 1948. Casing removed after well completed.

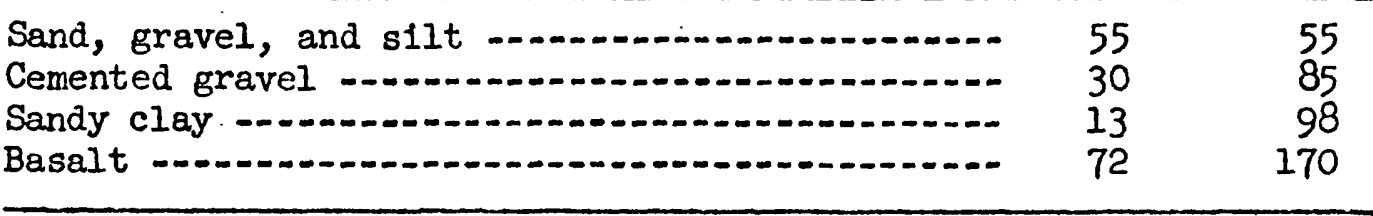


11/8-3Pl. U. S. Borax and Chemical Corp., well 2l. Altitude about 2,466 feet. Drilled by ower in April 1954. 14-inch casing zero to 304 feet, 10 -inch casing 304 to 430 feet.

Thiciness
(feet) $\begin{gathered}\text { Depth } \\ \text { (feet) }\end{gathered}$

Sand, clay, grevel; poorly sorted, moderately

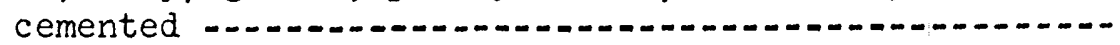

Clay; brown, sandy $91 \quad 91$

Sand, clay and gravel; sand, medium grain subrounded, quartz, feldspars, some basalt pebbles .........................

Sand, clay and gravel; sand medium-grained, subrounded, basalt, quartz and feldspar pebbles rariging to $25 \mathrm{~mm}$.,

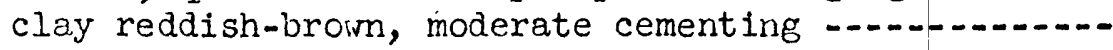

Gravel, sand and clay; gravel fine, well-sorted, clay reddish-brown as thin stringers ........... $13 \quad 182$

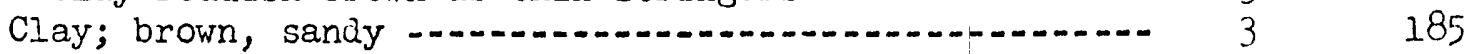

Gravel, sand and clay; clay reddish-brown possibly contalns some bentonite . 12

Basalt; brecciated or broken, black, contains some opalized material .......................... 35

Volcanic ash or tuff; reddish brown, bentonitic ...... 335

Basalt; brecciated or broken, reddish brown, cellular with secondary quartz filling .......... 6

Basalt; black, dense ............................. 41

Basalt; broken, brownish-red ...................... 13

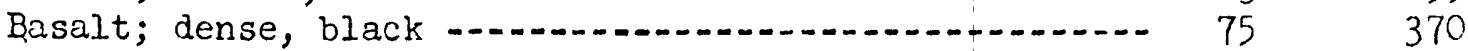

Basalt; broken, cellular ......................... $30 \quad 100$

Basalt; dense black ............................... $10 \quad 410$

Basalt, broken, soft, contains some fine-grained basaltic sand, possibly a filled fissure or cavity -- $4 \quad 414$

Basalt; broken, hard, black ...................... 2

Basalt; soft, black ............................... 3419

Basalt; dense, black, rard with few sort zones;

driller reports broken and softer basilt was

increasing down section ........ II 430

11/8-3QI. U. S. Borax and Chemical Corp., well 20. Altitude 2,472.5 feet. Drilled by owrer in March 1954. 16-inch casing zero to 266 feet, perforated interval uninown, uncased hole

from 266 to 414 feet.

Sand, clay and gravel, moderately vell cemented, occasional granitic boulders ..................... 115

Sand, clay and gravel, sand increasing, gravel

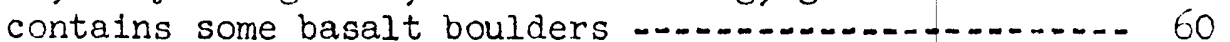

Gravel, sandy with streaks of brown clay .......... $10 \quad 185$

Clay and sand, sand fine grain, poorly sorted,

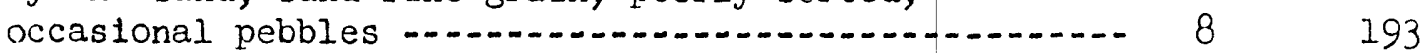

Gravel and sand, some brown clay ................. 12

Clay and sandy gravel, poorly sorted ............... 5210

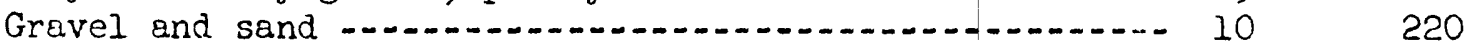

Clay, sandy with occasional granitic boulders ......... 5 
11/8-3Q1..--Continued. Material

Gravel and sand with some basalt boulders ............. $13 \quad 238$ Basalt breccia, broken, black, with some red zones .... $22 \quad 260$ Basalt, black, dense, alternate zones of easy and hard drilling ............................................. 80 Volcanic ash, white, bedded, tight, tuff ............... 54 Basalt, black, dense -................................. $20 \quad 414$

11/8-3Rl. U. S. Borax and Chemical Corp., well 24, Alt1tude about 2,1471 feet. Drilled by owner in May 1954. 12-Inch casing zero to 353 feet; 8-inch casing from 347 to 468 feet; tools in uncased hole from 468 to 500 feet.

Sand, clay and gravel; poorly sorted, moderately cemented, occasional boulders of basalt ............... 180

Sand and gravel with occasional streaks of reddish-brown clay -................................... 25

Gravel and sand; mostly quartz and feldspars, some basalt boulders -................................. 65

Basalt; black, broken ........................................ 7

Clay; sandy with some bentonfte, reddish-brown,

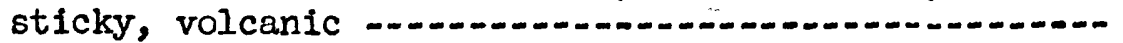

Basalt; broken, black ...................................

Basalt; broken with streaks of brown ash or clay .......-

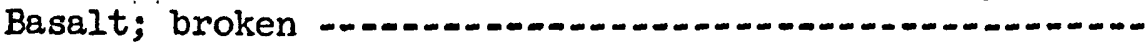

Clay, tuff or volcanic ash, reddlsh-brown,

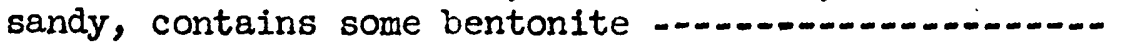

Basalt; broken ................................................

Basalt; broken with streaks of reddish-brown

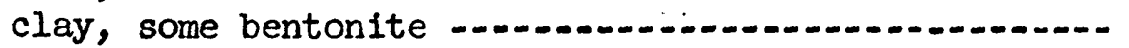

Basalt; dense, hard, black ................................

Clay; reddish-brown, sandy, volcanic ash or tuff, contains some bentonite .........................

Basalt; dense, hard, broken with few streaks of clay

Basalt; black, cellular, soft. Drillers report it contains some water ................................ 19

Basalt; black, with some streaks of ash or clay ........ 53

Clay; volcanic ash or tuff, reddish-brown, sandy, bentonitic -...................................... 10

Basalt; black, dense, hard ........................... 18

11/8-3-2, U. S. Borax and Chemical Corp, well 14. Altitude about 2,500 feet. Drilled by owner in 1948. Casing removed from well.

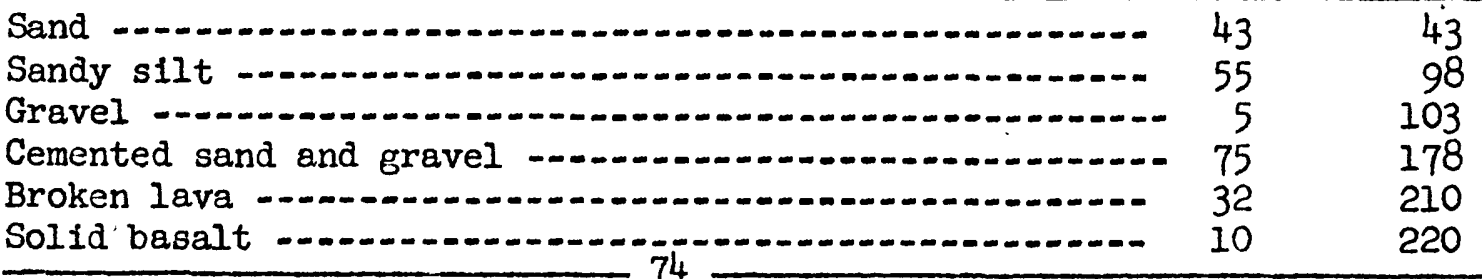


11/8-3-3. U. S. Borax and Chemical Corp., well 15. Altitude about 2,472 feet. Dr1lled by owner in 1948, casing removed from well.

Material

Thickness

(feet)

Depth

(feet)

Cemented sand and gravel (3-ft seam of loose gravel at $64 \mathrm{ft})$

Sandy clay and gravel

Loose water-bearing gravel

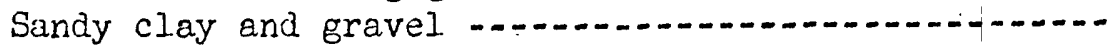

Cemented gravel and basalt boulders ....

Broken baselt .......

Basalt - . .

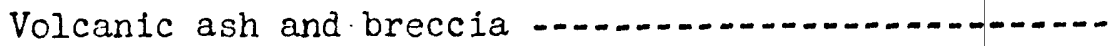

Basalt ...........

$115 \quad 115$

$60 \quad 175$

$10 \quad 185$

$40 \quad 225$

$10 \quad 235$

$7 \quad 242$

$97 \quad 339$

$68 \quad 407$

$7 \quad 414$

11/8-10PI. U. S. Borax and Chemical Corp., well 10. Altitude about 2,435 feet. Drilled by owner in 1948. 10-1nch casing.

\begin{tabular}{|c|c|c|}
\hline granite & 95 & 95 \\
\hline Cemented gravel & 14 & 109 \\
\hline Baisalt & 65 & 174 \\
\hline Mixed basalt and sand clay & 9 & 183 \\
\hline Basalt $\ldots \ldots+\ldots \ldots$ & 6 & 189 \\
\hline Broken basalt & 20 & 209 \\
\hline Brown sand, clay, and basalt & 30 & 239 \\
\hline
\end{tabular}

11/8-10-1. U. S. Borax and Chemical Corp., well 11. Altitude about 2,435 feet. Drilled by owner in 1948 .

Decomposed granite ........... 80

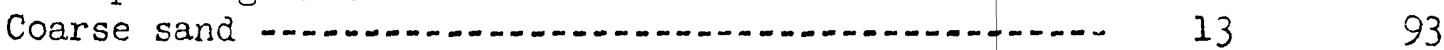

Cemented gravel ................................... $47 \quad 140$

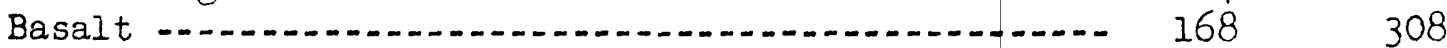

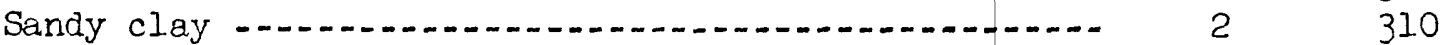

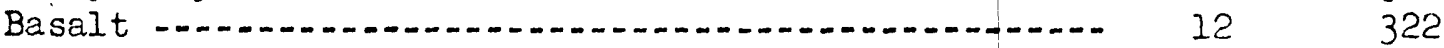

II/8-IIDI. U. S. Borax and Chemical Corp., vell 7. Altitude about 2,480 f'eet. Drillea by orner in 1945. 14-1nch casing.

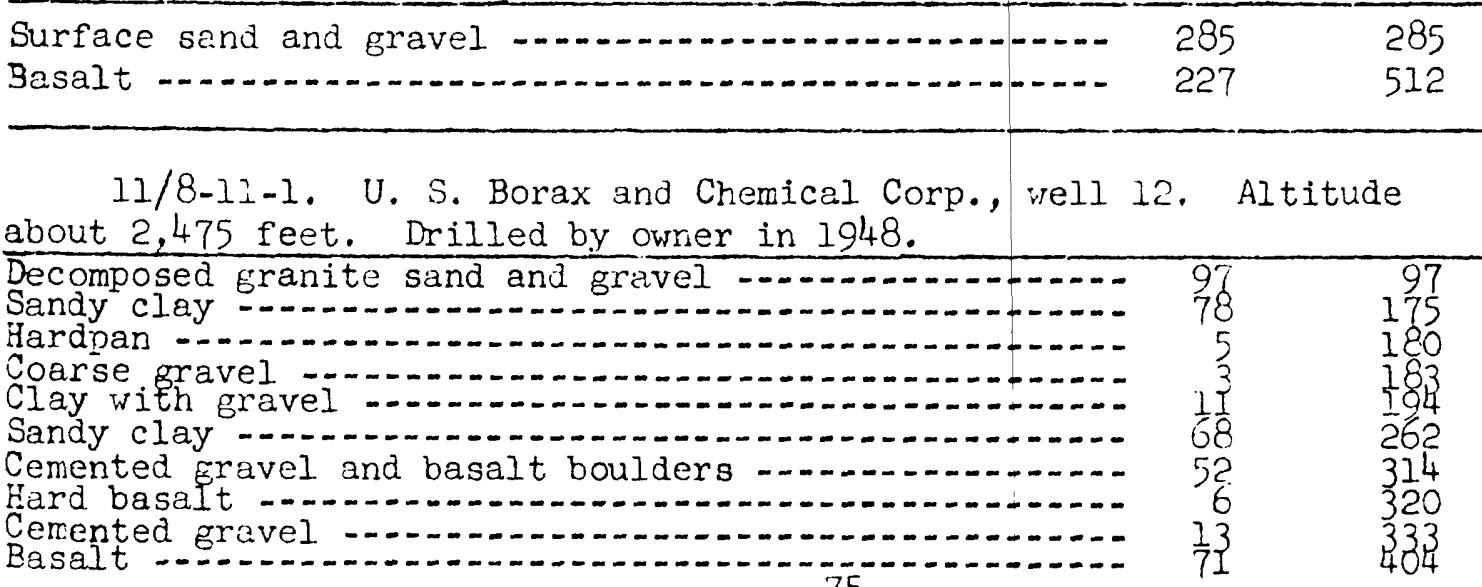

Basalt _._. 
11/8-15K. U: S. Borax and Chemical Corp., well 26. Altitude about 2,426 feet. Drilled by Mogle Brothers in September 1954. 14-1nch casing zero to 483 feet, perforated 170 to 230 and 360 to 400 feet, uncased hole 483 to 678 feet.

$\frac{400 \text { feet, unickness Depth }}{\text { Material. Thet }}$

Topsoil; sandy, light brown, with some gravel ....... is (feet) (feet)

Sand and clay; sand, coarse.to very coarse, buff, subangular, clay, light brown

Sand, clay and gravel;- well mixed, compact, light brown

Clay; light brown

Sand, clay and gravel; sand medium to coarse, clay light brown, gravel up to $3 / 4$ inch containing

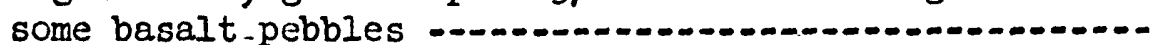

Clay; sandy, gray, micaceous

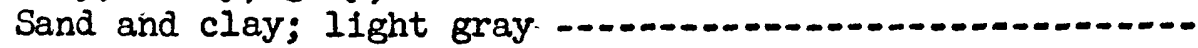

Limestone; white to light gray, hard, siliceous, possibly indurated old caliche zone

Sand; coarse grain, gray, some pebbles

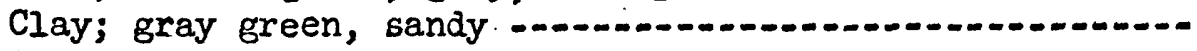

Limestone;" white to "light gray, hard, siliceous, possibly indurated old caliche zone

Clay; gray green -..........

Limestone; gray, possibly large boulder

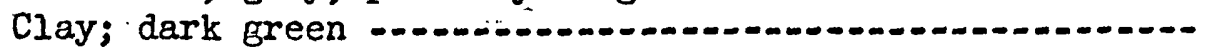

Iimestone; white to gray, hard, secondary calcite and nodules and bands of siliceous material

Shale; gray green, hard, dry

Shale; gray green, with inclusions of volcanic ash or pumice. Inclusions subrounded to rounded make up to 70 percent of sample

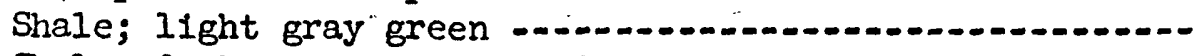

Shale; dark green, some banding

Sandstone; gray to buff, calcareous, silty, hard;

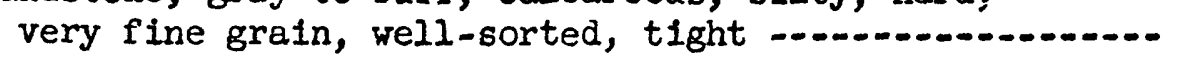

Shale; dark gray brown, banded, carbonaceous, micaceous

Shale; gray green, streaks of yellow material ...........

Shale; bentonitic, contains volcanic debris, possibly pumice

Limestone; tan, dolomitic, siliceous, hard,

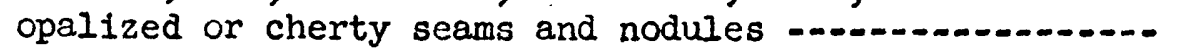

Shale and sandstone; shale light green, sandstone

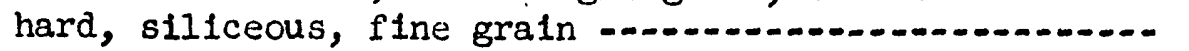

Shale; dark g ray green, compact

Shale; sandy with inclusions of basalt and scoria

Limestone; white, sandy, hard, possibly Indurated

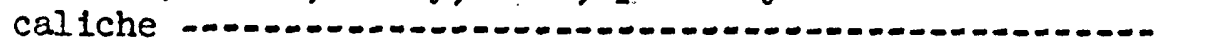

Shale; Ilght gray green, fractured, siliceous

Sandstone; dark gray-green, hard, containing

pebbles of cellular basalt, siliceous ............ $16 \quad 360$

Clay; brown and coarse gravel, some basalt grains ....... $23 \quad 383$ 


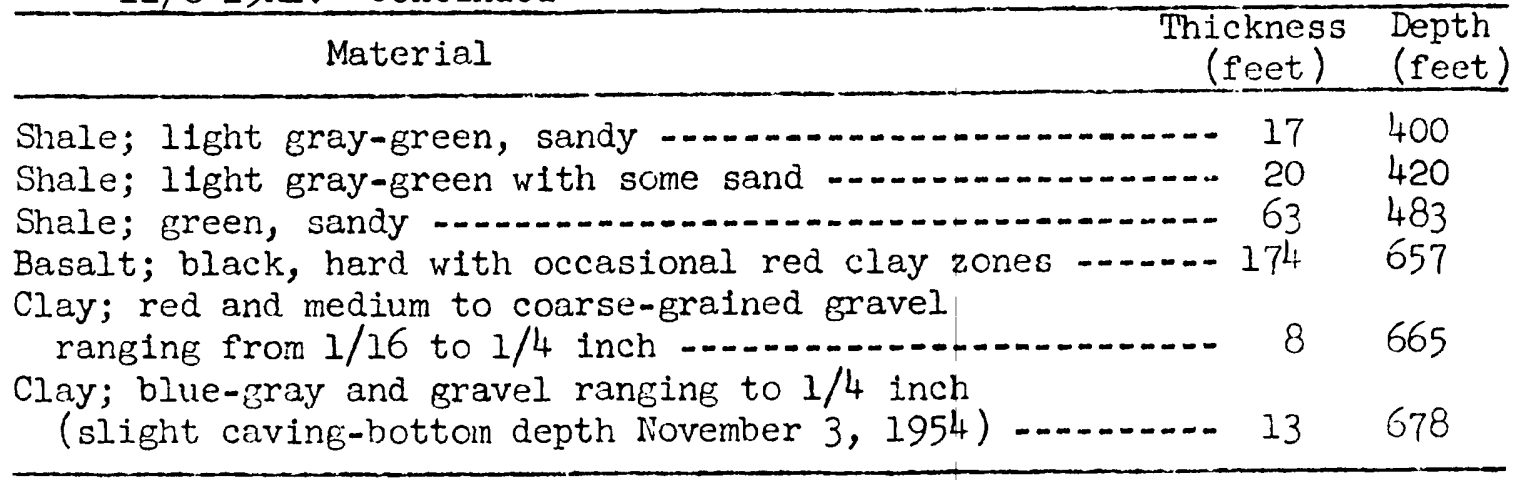

11/8-16Cl. U. S. Borax and Chenical Corp., well 9. Altitude about 2,430 feet. Dilled by owner in 1948. 16-inch uncased hole.

Alluvium
Gravel and boulders
Sand and clay
Ash and basalt
Basalt and water seepage
Basalt $\ldots$

11/8-16DI. U. S. Borax and Chemical Corp., well 8. Altitude about 2,420 feet. Drilled by ormer in 1948. Uncased hoie.

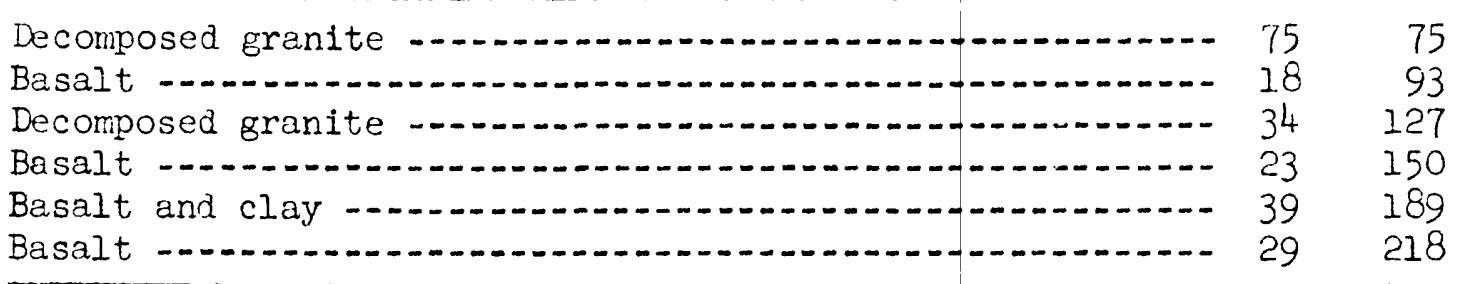

11/8-17R]. Garrett Corp., Rex Division. Altitude about 2,395 feet. Drilled by Rottman Drilling Co. in May 1957. 6-inch casing, perforated 181 to 366 feet.

\begin{tabular}{|c|c|c|}
\hline 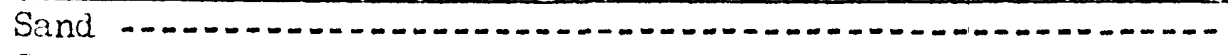 & 20 & 20 \\
\hline Sand-rock - & 20 & 40 \\
\hline Cemented sand & 50 & 90 \\
\hline Coarse sand cemented & 13 & 103 \\
\hline Coarse sand-inard rock & 20 & 123 \\
\hline Hard packed gravel-rock & 10 & 133 \\
\hline Boulders & 13 & 146 \\
\hline Hard cemented sand & 4 & 150 \\
\hline 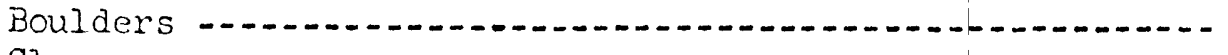 & 4 & 154 \\
\hline 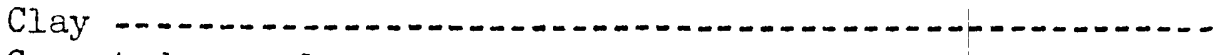 & 4 & 158 \\
\hline Cemented Gravel ...... & 8 & 166 \\
\hline Rock - & 10 & 176 \\
\hline iJand rock -- & 36 & 212 \\
\hline Broken rock-shale & 4 & 216 \\
\hline Hard black rock & 30 & 246 \\
\hline Soft lava rock & $\begin{array}{l}10 \\
\text { Cor }\end{array}$ & $\begin{array}{c}256 \\
\text { nued }\end{array}$ \\
\hline
\end{tabular}


$11 / 8-17 \mathrm{Rl}$.-.-Continued

\begin{tabular}{|c|c|c|}
\hline Materlal & $\begin{array}{l}\text { Thickness } \\
\text { (feet) }\end{array}$ & $\begin{array}{l}\text { Depth } \\
\text { (feet) }\end{array}$ \\
\hline $\begin{array}{l}\text { Soft lava rock-sand } \\
\text { Black lava-soft gray streaks } \\
\text { Lava rock-hard } \\
\text { Softer rock-sand streaks } \\
\text { Hard black rock }\end{array}$ & $\begin{array}{rr}-. & 9 \\
-- & 32 \\
- & 59 \\
- & 2 \\
-- & 9\end{array}$ & $\begin{array}{l}265 \\
297 \\
356 \\
358 \\
367\end{array}$ \\
\hline
\end{tabular}

11/8-19Ll. U. S. Borax and Chemical Corp., well 42. Alt1tude about 2,350 feet. Drilied by Reliance Drilling Co. in August 1955. 12-Inch casing zero to 361 feet; perforated 96 to 361 feet.

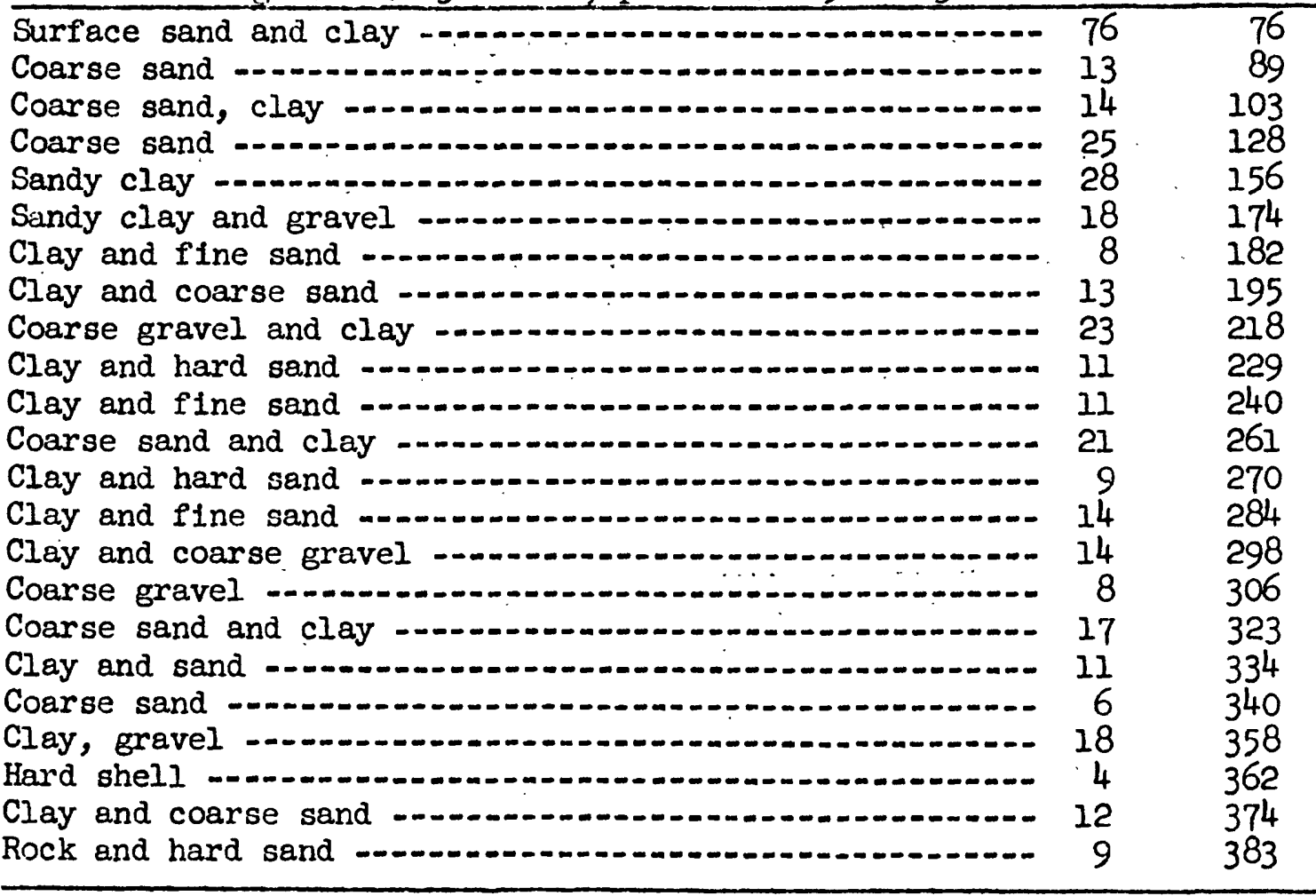

11/8-26Al. C. J. "Roycroft. Alt1tude about 2,425 feet. Drilled by Pacific Coast Borax Co. In 1927, redrilled by ormer in 1956. 5-Inch casing zero to 321 feet, perforated 281 to 321 feet; uncased hole 321 to 500 feet, 6-inch casing 500 to 950 feet, uncased hole 950 to 1,100 feet.

Granite 
11/8-29KI. U. S. Borax and Chemical Corp., well 41. Altitude about 2,355 feet. Drilled by Reliance Drilling Co. in August 1955. 12-inch casing zero to 495 feet; perforated 96 to 495 feet.

\begin{tabular}{|c|c|}
\hline Material & $\begin{array}{l}\text { Depth } \\
\text { (feet) }\end{array}$ \\
\hline urface sand and clay & 96 \\
\hline Medium sand & 107 \\
\hline Coarse sand and clay .. & 127 \\
\hline ine sand, clay & 143 \\
\hline Hard sand & 159 \\
\hline Lay, coarse gravel -- & 172 \\
\hline Clay and boulders .... & 195 \\
\hline Hard sand and clay ... & 217 \\
\hline Clay, fine sand ....... & 229 \\
\hline Vlay, coarse sand ..... & 240 \\
\hline Coarse sand and clay .. & 258 \\
\hline Coarse sand & 271 \\
\hline Doarse sand and clay. & 285 \\
\hline 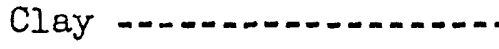 & 306 \\
\hline ine sand and clay .- & 325 \\
\hline Coarse gravel & 336 \\
\hline Coarse gravel and clay & 368 \\
\hline Coarse gravel & 374 \\
\hline Coarse sand and clay & 396 \\
\hline Caarse gravel & 403 \\
\hline Coarse gravel and clay & 427 \\
\hline andy clay and gravel - & 452 \\
\hline Coarse sand & 458 \\
\hline Coarse gravel and clay & 473 \\
\hline Coarse gravel & 482 \\
\hline Clay and rock & 495 \\
\hline
\end{tabular}

11/8-3021. U. S. Borax and Chemical Corp., well 32. Altitude about 2,335 feet. Drilled by Reliance Drilling Co. in July 1955. 12-inch casing zero to 485 feet, perforated 96 to 485 feet.

Surface sand and clay
Gravel and coarse sand
Gravel and clay
Hard sand
Coarse sand
Hard sand and clay
Small gravel clay
Flne sand and gravel
Clay and fine sand
Gravel and clay - sand
Clay and coarse sand
Coarse gravel-sand
Gravel and coarse sand
Gravel and clay -.


11/8-30Q1.--Cont1nued

\begin{tabular}{|c|c|c|}
\hline Material & $\begin{array}{l}\text { hickness } \\
\text { (feet) }\end{array}$ & $\begin{array}{l}\text { Depth } \\
\text { (feet) }\end{array}$ \\
\hline $\begin{array}{l}\text { Clay, coarse sand } \\
\text { Coarse gravel } \\
\text { Clay and coarse sand } \\
\text { Fine sand and clay } \\
\text { Clay and coarse gravel } \\
\text { Clay } \\
\text { Clay and fine sand } \\
\text { Clay, gravel } \\
\text { Clay } \\
\text { Coarse grevel and sand } \\
\text { Coarse sand and clay } \\
\text { Cemented sand -ave } \\
\text { Coarse gravel and clay } \\
\text { Gravel and sand } \\
\text { Coarse gravel and clay } \\
\text { Coarse sand - a } \\
\text { Cemented sand ard rock } \\
\text { Cemented sand rock } \\
\text { Rock and cemented sand } \\
\text { Cement, sand, rock }\end{array}$ & $\begin{array}{r}14 \\
9 \\
21 \\
22 \\
12 \\
11 \\
19 \\
14 \\
11 \\
14 \\
14 \\
10 \\
12 \\
8 \\
20 \\
5 \\
15 \\
12 \\
6 \\
19\end{array}$ & $\begin{array}{l}231 \\
240 \\
261 \\
283 \\
295 \\
306 \\
325 \\
339 \\
350 \\
364 \\
378 \\
388 \\
400 \\
408 \\
428 \\
433 \\
448 \\
460 \\
466 \\
485\end{array}$ \\
\hline
\end{tabular}

11/8-3IPI. U. S. Borax and Chemical Corp., well 39. Altitude about 2,325 feet. Drilled by Reliance Drilling Co. in August 1955. 12-inch casing zero to 303 feet; perforated 96 to 303 feet.

\begin{tabular}{|c|c|c|}
\hline \multirow{2}{*}{$\begin{array}{l}\text { Surface sand and clay } \\
\text { Medium sand }\end{array}$} & 96 & 96 \\
\hline & 12 & 108 \\
\hline Coarse sand and clay & 19 & 127 \\
\hline Coarse sand & 12 & 139 \\
\hline Coarse sand and clay ..... & 22 & 161 \\
\hline Coarse gravel & 11 & 172 \\
\hline Coarse gravel and clay - & 26 & 198 \\
\hline Coarse gravel & 9 & 207 \\
\hline Coarse gravel and clay. & 20 & 227 \\
\hline Coarse gravel and rock .... & 9 & 236 \\
\hline Medium sand and gravel .... & 8 & 244 \\
\hline Coarse gravel and clay & 24 & 268 \\
\hline Hard sand and rock & 4 & 272 \\
\hline Rock and cemented sand .... & 18 & 290 \\
\hline Cemented sand & 10 & 300 \\
\hline Rock $\ldots-\cdots$ & 3 & 303 \\
\hline
\end{tabular}


11/8-33Ll. California Borax Co. Alt1tude about 2,345 feet. Drilled by R. C. Clay in Janaury 1958. 10-inch casing zero to 500 feet; perforated 290 to 320 and 383 to 485 feet.

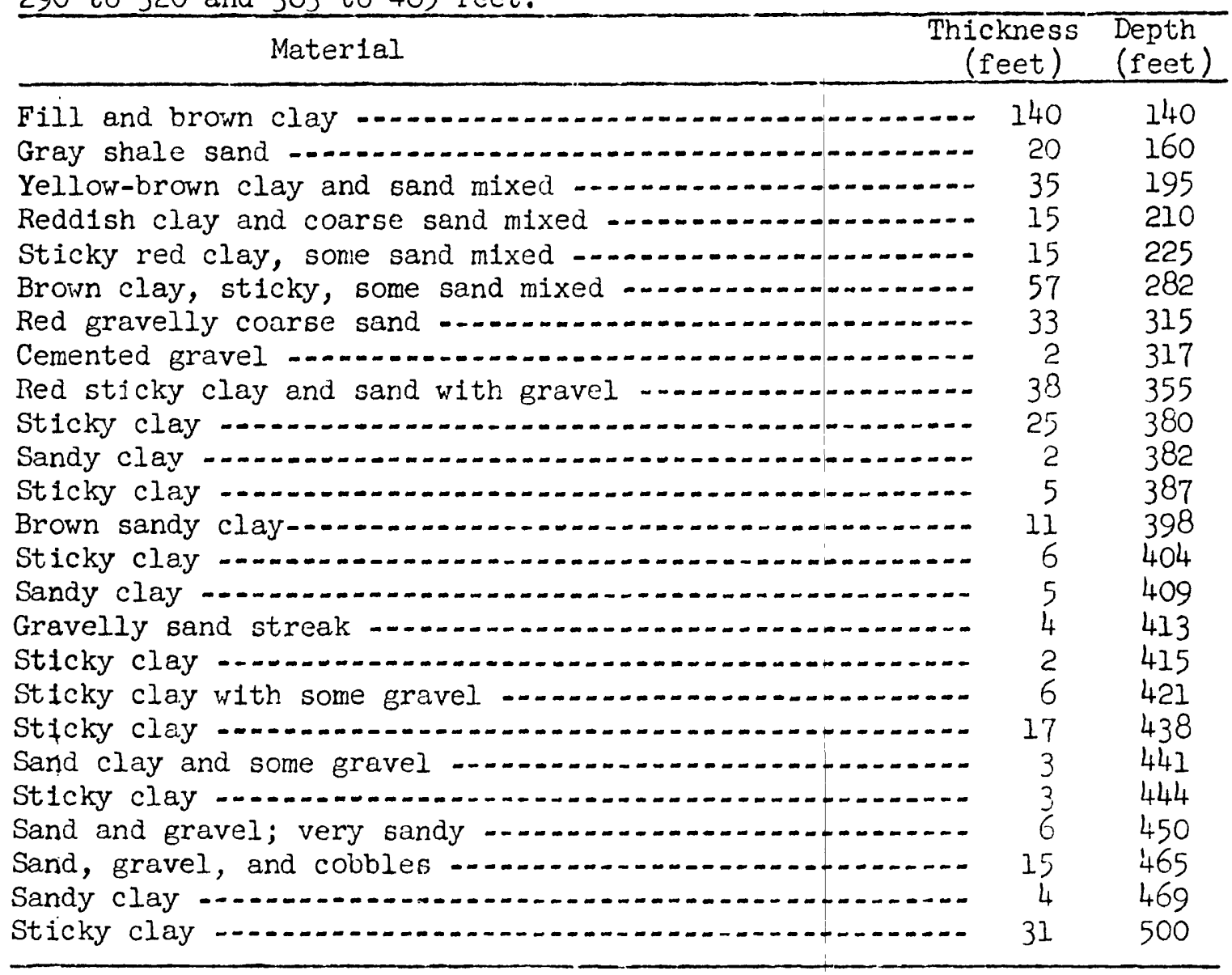

11/8-35DI. H. B. Hays, Desert Lake. Altitude about 2,380 feet. Drilled by Reliance Drilling; Co. in August 1955. 12-inch casing zero to 606 feet; perforated 96 to 606 feet.

\begin{tabular}{|c|c|c|}
\hline Surface sand and clay $\ldots \ldots$ & 68 & 68 \\
\hline Coarse sand and clay & 13 & 81 \\
\hline Medium sand & 15 & 96 \\
\hline Coarse sand and clay & 16 & 112 \\
\hline Coarse sand & 8 & 120 \\
\hline Coarse sand and ciay & 17 & 137 \\
\hline 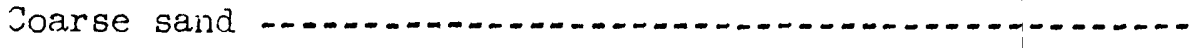 & 5 & 142 \\
\hline 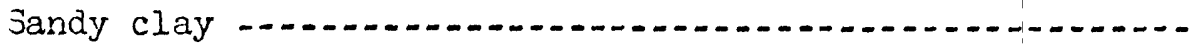 & 21 & 163 \\
\hline Ilay, streaks hard shell -. & 17 & 180 \\
\hline Medium sand & 9 & 189 \\
\hline Medium sand and clay & 17 & 206 \\
\hline Zoarse sand & 5 & 211 \\
\hline Zoarse sand and clay ............ & 15 & 226 \\
\hline 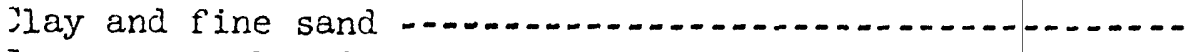 & 12 & 238 \\
\hline 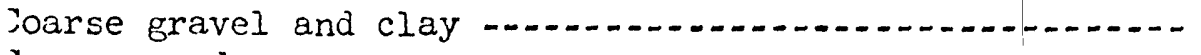 & 13 & 251 \\
\hline Joarse sand & $\begin{array}{l}16 \\
\text { Cor }\end{array}$ & $\begin{array}{l}267 \\
\text { ded }\end{array}$ \\
\hline
\end{tabular}


$11 / 8-35 \mathrm{Dl}$.--Continued

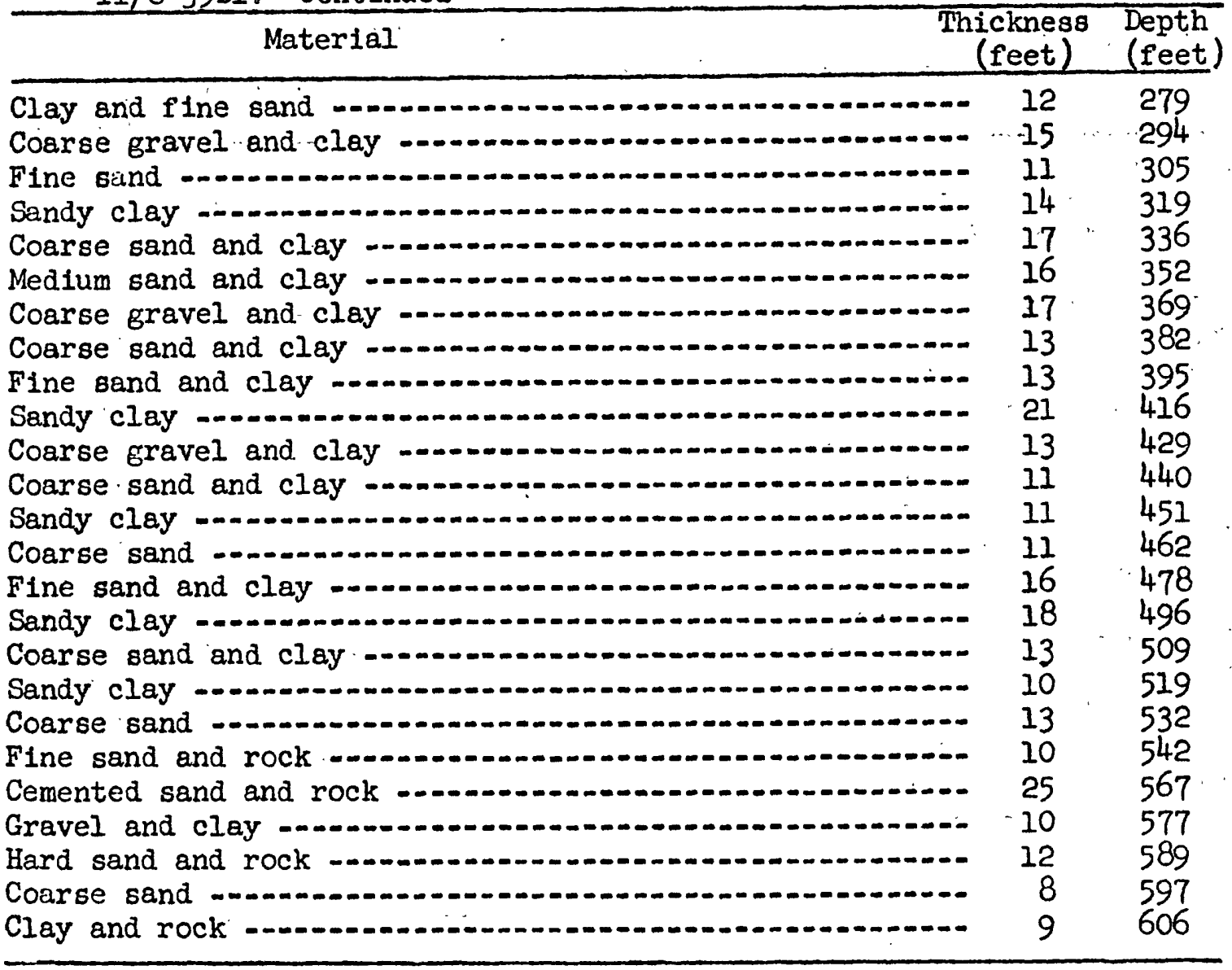

11/9-13DI. U. S. Borax and Chemical Corp., well 40. Altitude about 2,375 feet. Drilled by Rellance Drilling Co. in August 1955. 12 -inch casing zero to 312 feet; perforated 96 to 312 feet.

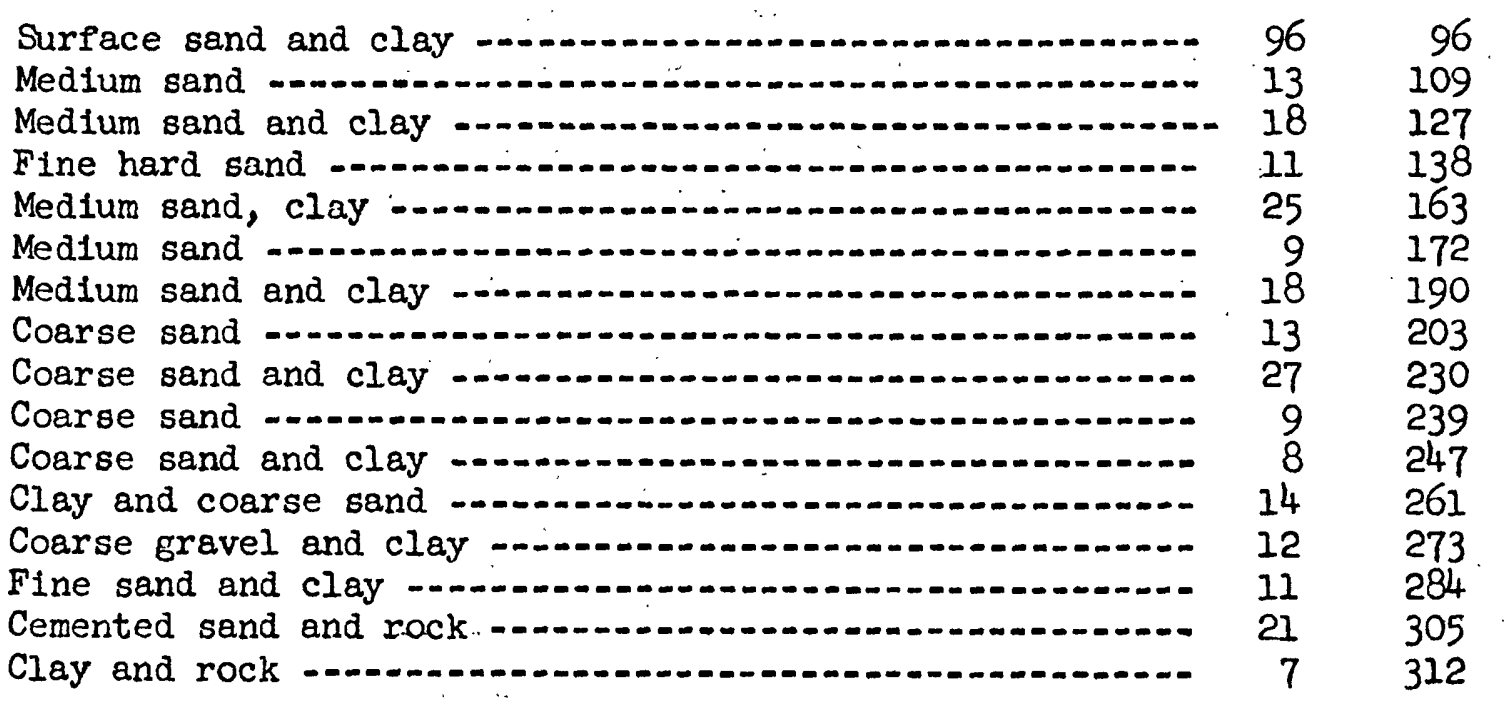


11/9-13Ll. U. S. Borax and Chemical Corp., well 45. Altitude about 2,360 feet. Drilled by Rellance Drilling Co. in August 1955. 12-1nch casing zero to 462 feet; perforated 144 to 462 feet.

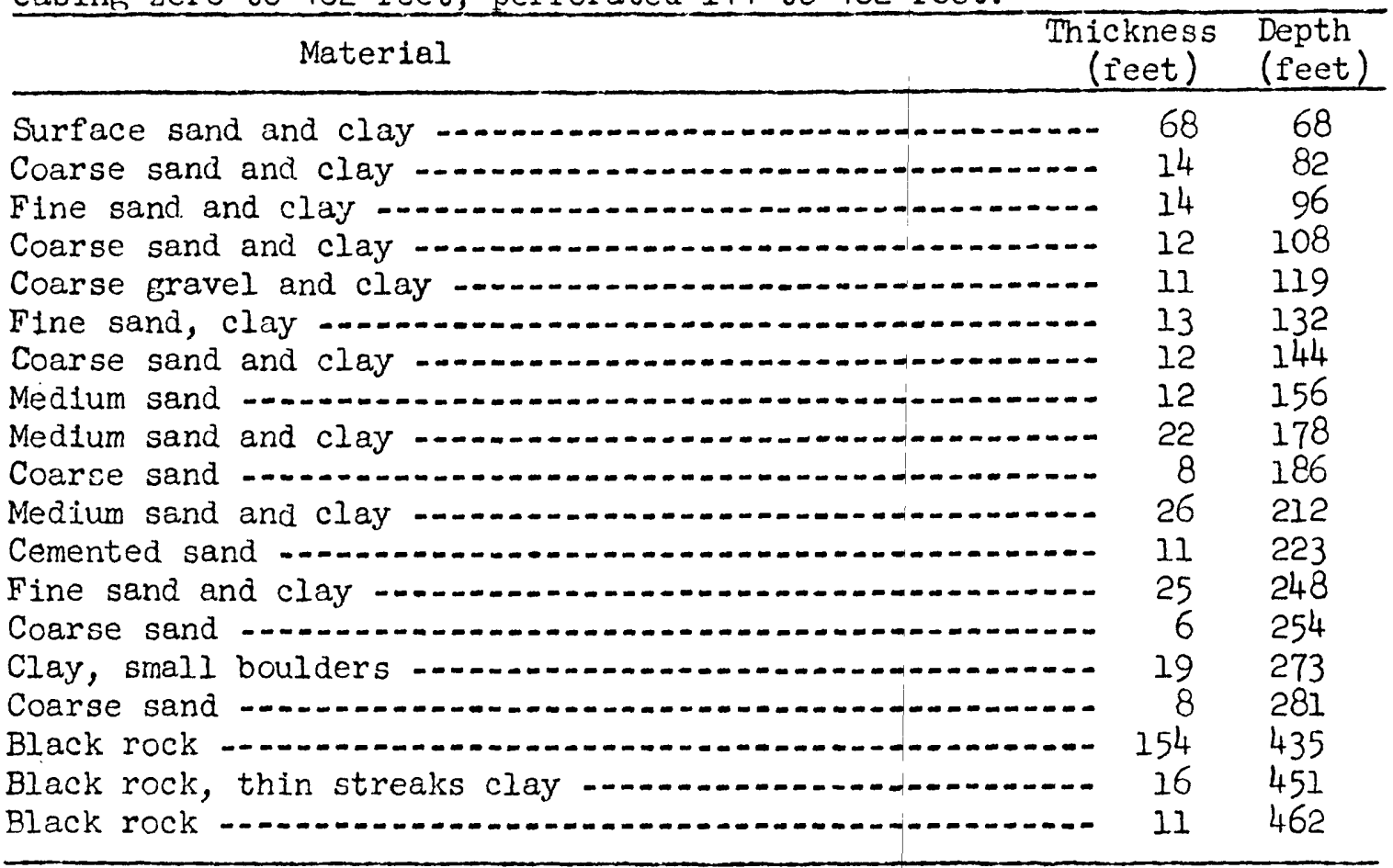

11/9-24Al. U. S. Borax and Chemical Corp., well 27. Altitude 2,348.8 feet. Drilled by Roscoe Moss Drilling Co. in April 1955. 16-inch casing zero to 888 feet; perforated 200 to 870 feet, uncased hole 888-900 feet.

Topsoil; sandy, light brown ................... 11

Sand and clay; light brown, few pebbles and boulders, sand fine to very coarse grain, subangular, quartz and feldspars predomirant with occasional pebbles of dark reddish-brown basalt ....................... $107 \quad 108$

Clay; sandy, light brown, sand fine grain,

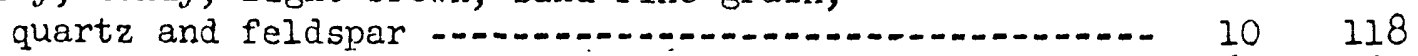

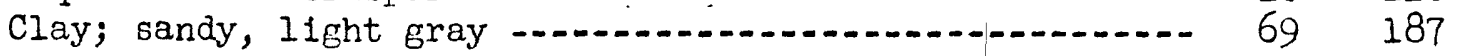

Send, clay and gravel; light brown, sand very coarse, gravel up to 1 inch well rounded .......... 5192

Clay; light brown, sandy with few pebbles of granitic material ...................................... 300

Clay and sand; light brown, hard ......................... 48 348

Sand, clay and gravel; light brown, poorly sorted, sand fine to coarse grain, pebbles and boulders of basalt and granitic materfal subrounded .......... 2350

Sand and clay; light brown, hard, some basalt pebbles ...- $20 \quad 370$

Sand, gravel and clay; sand fine to very coarse, quartz and feldspars predominant, gravel mostly basalt, scoria and other volcanic-flow material ...... 18388 
$11 / 9-24$ Al. - - Continued

\begin{tabular}{cc}
\hline Material & Thickness \\
(feet) & Depth \\
(feet)
\end{tabular}

Sand, clay and gravel; brown, sand very coarse, gravel mostly basalt and other flow material, poorly sorted

Basalt; broken, soft, clay filling in cracks and

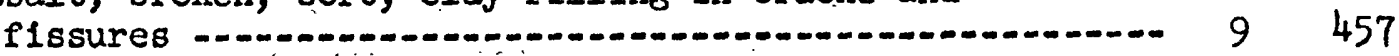

Sand, gravel and clay; very coarse sand and basalt pebbles poorly sorted in a clay matrix .................. $18 \quad 475$

Sand, clay and gravel; hard, cemented, coarse mater1al mostly basalt and granit1c material ............. $33 \quad 508$

Clay; light brown, sandy, hard ............................ 92600

Clay and sand; Ilght brown, hard with occasional pebbles up to I Inch ...................................... $140 \quad 740$

Sand clay and gravel; light gray-green, sand very coarse, 10 percent to 20 percent of sample composed of subrounded basaltic and granitic pebbles ranging up to 2 -inch diameter. Clay and sand contains some volcan1c ash ......................... $55 \quad 795$

Sand and gravel; sllty, composed of basalt pebbles and boulders well cemented in a sandy clay matr1x, contains some volcanic ash ........... $20 \quad 815$

Clay and sand; light brown some 1/4-inch gravel ........... $20 \quad 835$

Clay; sandy, light gray-green _............................... $25 \quad 860$

Clay; sandy, dark gray-green -................................ $30 \quad 890$

Sand and clay; gray-green with yellowish-brown zones, sand mostly volcanic debris "bottom" ............... 10900

11/9-24B2. U. S. Borax and Chemical Corp., well 43. Altitude about 2,345 feet. Drilled by Rellance Drilling Co. In July 1955. 12-1nch casing zero to 542 feet; perforated 96 to 542 feet.

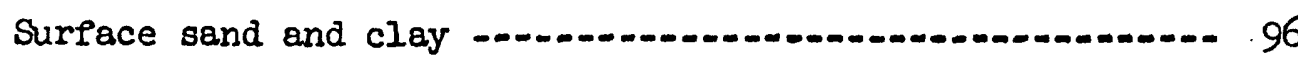

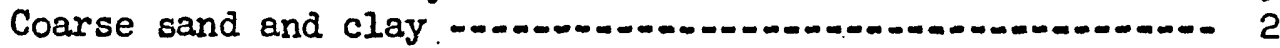

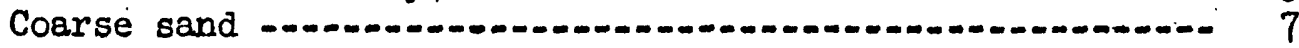

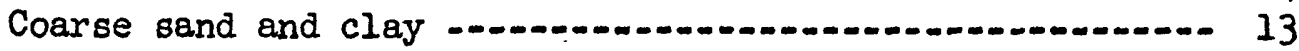

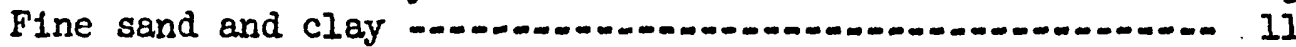

Hard sand and clay

Coarse gravel and clay.

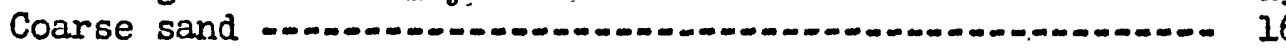

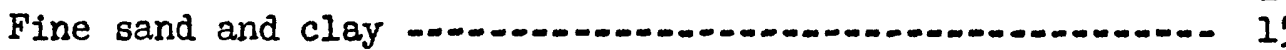

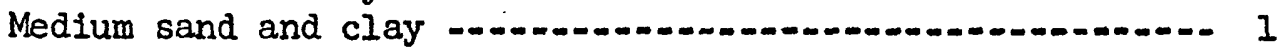

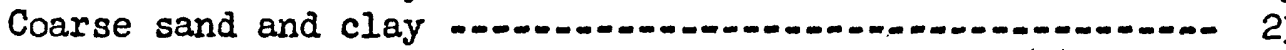

Coarse gravel, clay -and

Boulders and clay -.... 1

Coarse sand ............

Coarse sand and clay -................... 18

Clay and fine sand

Clay and coarse sand .............

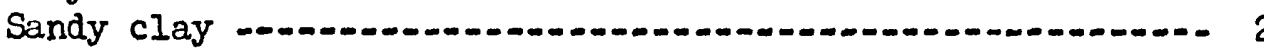

96

119

7126

139

11.150

9159

3172

16188

5203

3216

239

21260

11271

$17 \quad 288$

18306

15321

29350

$22 \quad 372$

Continued 
$11 / 9-24 B 2 .--$ - Continued

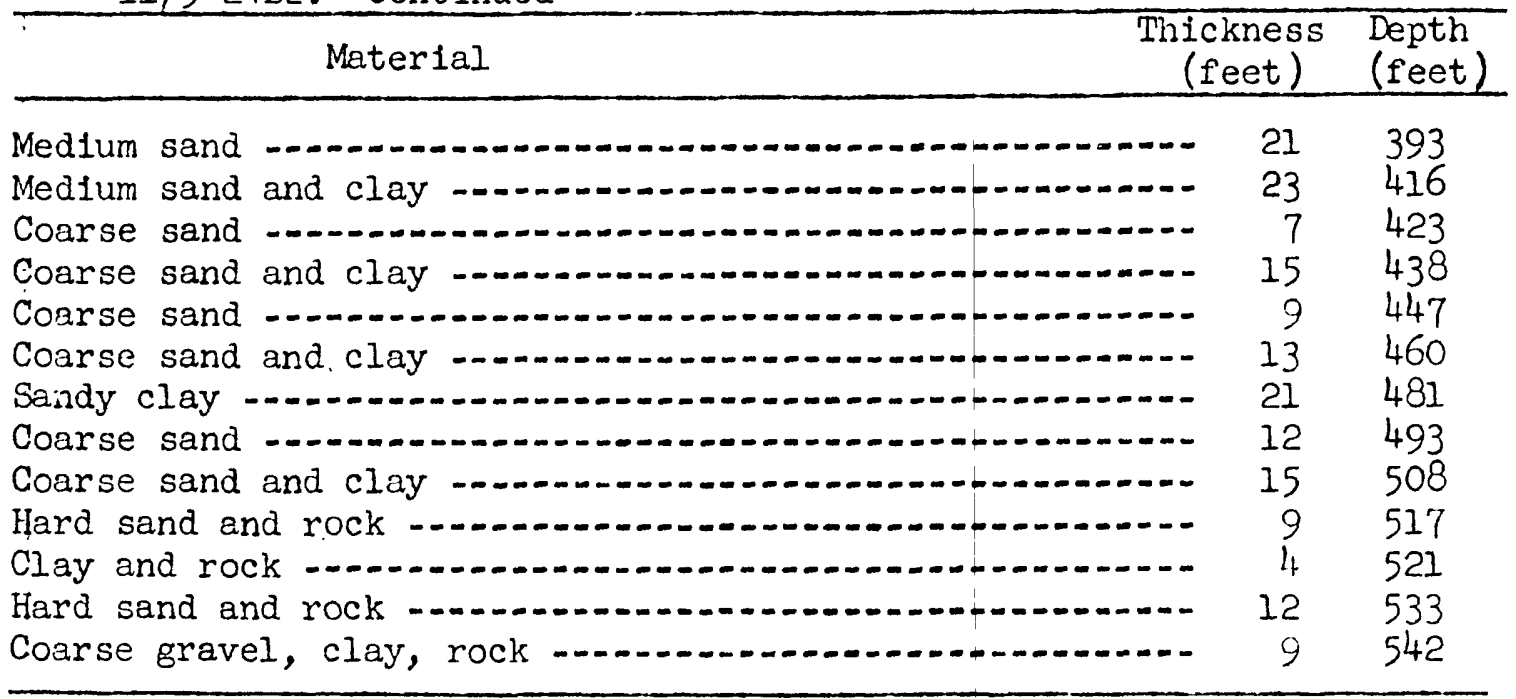

11/9-24Q1. U. S. Borax and Chemical Corp., well 33. Altitude about 2,335 feet. Drilled by Reliance Drilling Co. in July 1955. 12 -inch casing zero to 360 feet: perforated 96 to 360 feet.

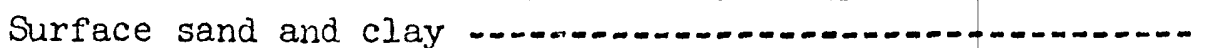

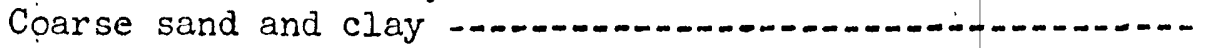

Coarse sand -........

Gravel and clay -..2.

Coarse sand -..._.

Sand and clay -.

Coarse sand and clay ................................ 13189

Medium sand ........................................... $12 \quad 201$

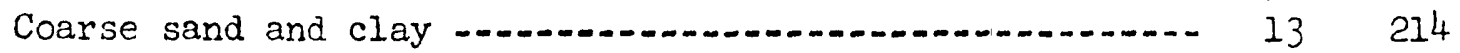

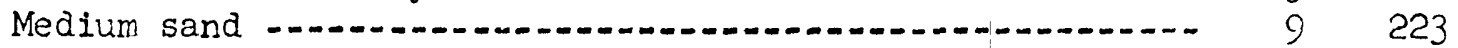

Coarse sand and clay ................................. 13 236

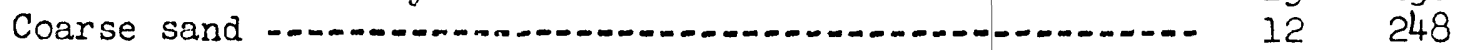

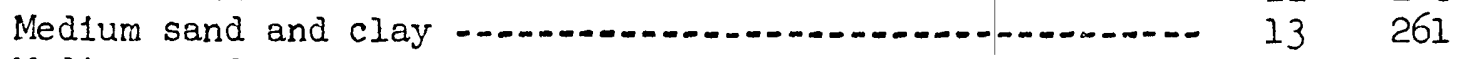

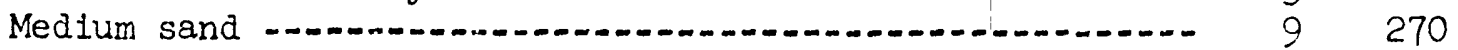

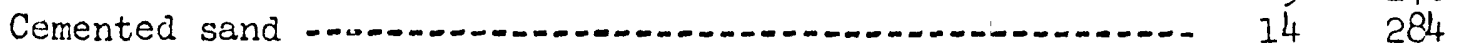

Cemented sand and gravel ........................... 992

Boulders and hard sand .................................. 12305

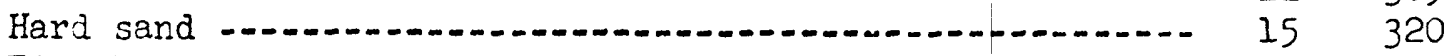

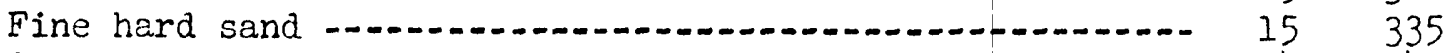

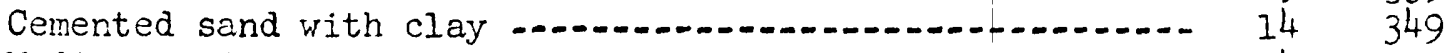

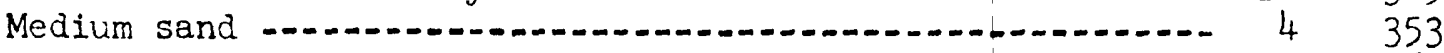

Clay, sand (n) 760 
11/9-25L1, U. S. Borax and Chemical Corp., well 34. Altitude about 2,330 feet. Drilled by Reliance Drilling Co. in July 1955. 12-1nch casing zero to 480 feet; perforated 96 to 480 feet." Material Thickness Depth

Surface sand and clay

Coarse gravel with clay Coarse sand and clay

Fine sand and clay

Coarse gravel and clay

Coarse sand and clay

Fine sand and clay

Hard sand and clay

Coarse gravel, sand

Hard sand

Coarse sand and clay

Coarse gravel and clay

Coarse sand and clay

Hard sand -........

Fine sand and clay

Medium sand and clay ....

Clay and boulders

Hard sand and clay

Clay and fine sand

Coarse sand and clay

Medium sand ..........

Gravel and clay

Coarse gravel ........

Gravel, clay -..

Cemented sand -

Gravel and clay

Clay and small boulders

Sand and gravel

Coarse sand and clay

Clay and sand -......

Medium sand -...-...

Clay with coarse gravel

Hard shell -......

(feet) (feet)

$\begin{array}{rr}64 & 64 \\ 12 & 76 \\ 22 & 98 \\ 18 & 116 \\ 16 & 132 \\ 18 & 150 \\ 11 & 161 \\ 11 & 172 \\ 10 & 182 \\ 12 & 194 \\ 15 & 209 \\ 16 & 225 \\ 15 & 240 \\ 16 & 256 \\ 8 & 264 \\ 15 & 279 \\ 14 & 293 \\ 14 & 307 \\ 10 & 317 \\ 8 & 325 \\ 11 & 336 \\ 14 & 350 \\ 11 & 361 \\ 16 & 377 \\ 7 & 384 \\ 12 & 396 \\ 10 & 406 \\ 13 & 419 \\ 8 & 427 \\ 7 & 434 \\ 9 & 443 \\ 17 & 460 \\ 9 & 469 \\ 8 & 477 \\ 3 & 480 \\ \end{array}$

11/9-28Cl. Harry Levy. Altitude about 2,305 feet. Drilled by Pacific Coast Borax Co. In April 1932. 5-inch casing.

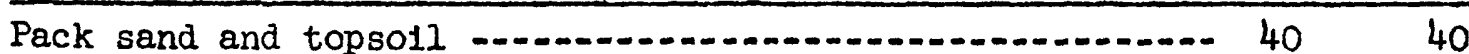

Gravel _...

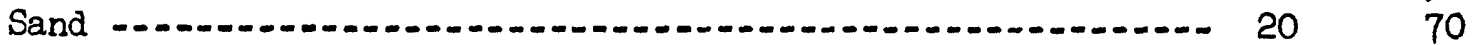

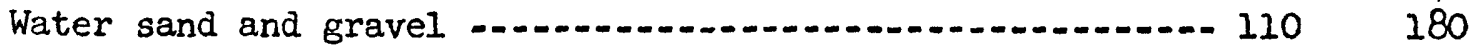

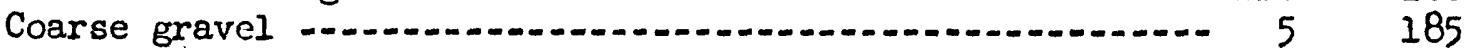

Brown shale ....... 5190

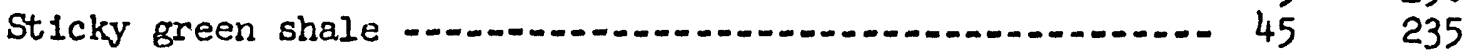

Continued 
11/9-28Cl. -.-Cont inued

\begin{tabular}{|c|c|c|}
\hline Material & $\begin{array}{c}\text { Tilickness } \\
\text { (feet) }\end{array}$ & $\begin{array}{l}\text { Depth } \\
\text { (feet) }\end{array}$ \\
\hline Brown shale & -- & 240 \\
\hline Dark gray shale & $\ldots$ & 260 \\
\hline Brown shale & $\ldots$ & 270 \\
\hline Dark gray shale ............ & 8 & 278 \\
\hline Granitic sand & $\ldots$ & 290 \\
\hline Gray sandy shale ....... & -- & 303 \\
\hline Blue shale & 23 & 326 \\
\hline Brown shale & $\ldots$ & 376 \\
\hline Blue shale & 9 & 385 \\
\hline Hard blue sandstone .... & 68 & 453 \\
\hline Blue sliale & 10 & 463 \\
\hline Hard blue sandstone .... & --- & 468 \\
\hline Blue-green shale ............ & $-\infty$ & 527 \\
\hline Hard dark gray sandstone & 148 & 675 \\
\hline Quartz $\ldots \ldots$ & 27 & 702 \\
\hline Hard blue sandstone & 6 & 708 \\
\hline 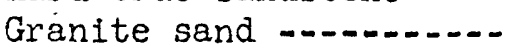 & 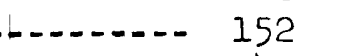 & 860 \\
\hline Hard blue sandstone .... & $\ldots$ & 900 \\
\hline Hard blue sandy shale -- & $-\ldots$ & 936 \\
\hline Hard brown sandy shale - & $\ldots-\ldots$ & 955 \\
\hline Blue sandy shale .......... & 15 & 970 \\
\hline Light gray sandy shale - & 76 & 1,046 \\
\hline Brown sandy shale & $-\infty$ & 1,060 \\
\hline Hard white sandstone ..- & $-\ldots$ & 1,072 \\
\hline Brown sandy shale & $-\ldots$ & 1,085 \\
\hline Gray sandy shale ........ & $-\ldots$ & 1,125 \\
\hline Brown sandy shale & $-\ldots$ & 1,360 \\
\hline Gray sandy shale & 50 & 1,210 \\
\hline Granite sand & $-\ldots-\ldots$ & 1,340 \\
\hline
\end{tabular}

11/9-29HI. U. S. Borax and Chemical Corp., well 37. Altitude about 2,305 feet. Drilled by Reliance Irilling Co. in July 1955. 12-inch casing zero to 395 feet; perforated 96 to 395 feet.

\begin{tabular}{|c|c|c|}
\hline Surface sand and clay & 58 & 58 \\
\hline Coarse sand & 13 & \\
\hline Voarse sand and gravel & 17 & 88 \\
\hline Zoarse sand and clay & 15 & 103 \\
\hline Slay and gravel & 9 & 112 \\
\hline Zoarse gravel and clay & 14 & 126 \\
\hline 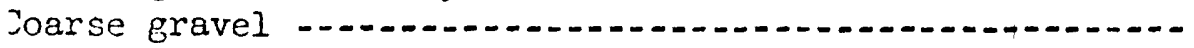 & 12 & 138 \\
\hline Joarse gravel and clay & 18 & 156 \\
\hline Voarse sand, gravel & 7 & 163 \\
\hline Joarse sand and clay ........... & 17 & 180 \\
\hline iravel & 9 & 189 \\
\hline Iravel and clay & 17 & 206 \\
\hline 'Iay and sand & II & 217 \\
\hline Ooarse sand and clay & 11 & 228 \\
\hline ledium sand & $\begin{array}{l}13 \\
\text { Cor }\end{array}$ & 241 \\
\hline
\end{tabular}


11/9-29H1. .-. Continued

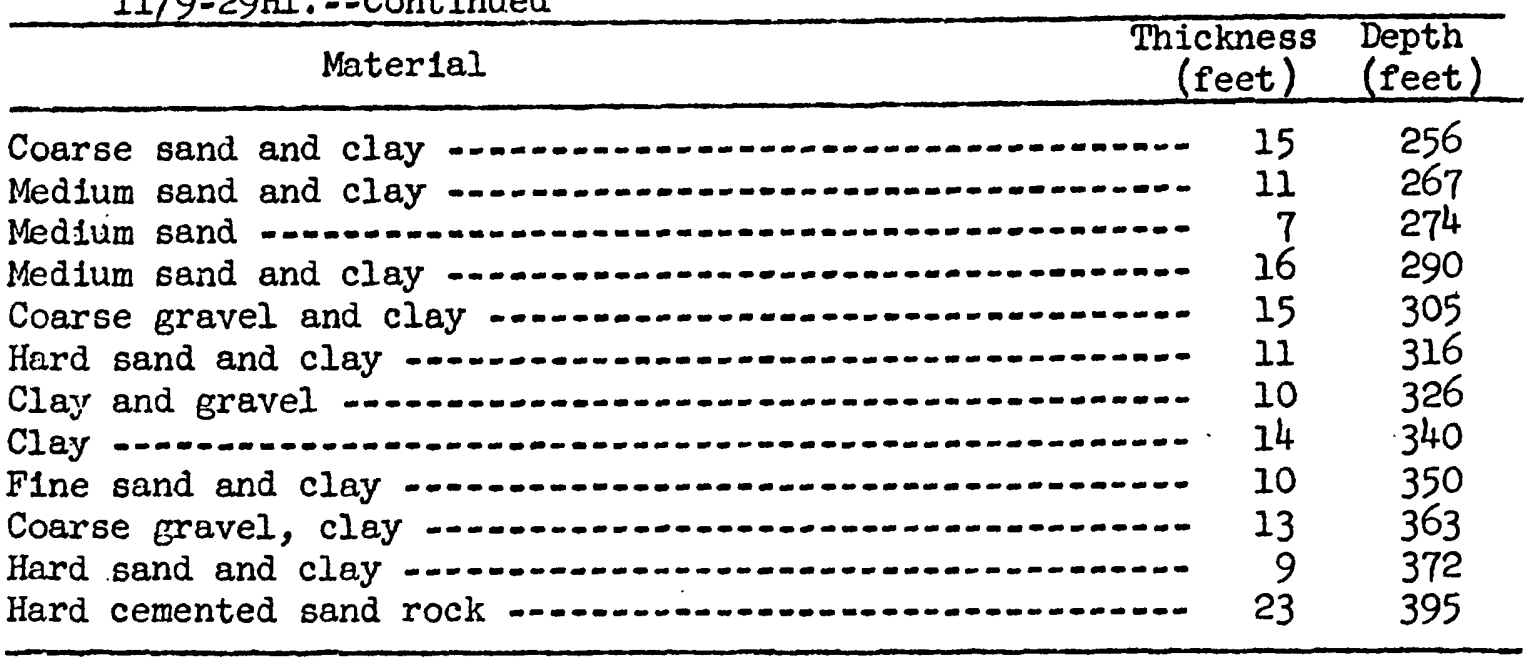

11/9-29K. U. S. Borax and Chemical Corp., well 38. Altitude about 2,300 feet. Drilled by Rellance Drilling Co, in July 1955. 12-1nch casing zero to 405 feet; perforated 96 to 405 feet.

\begin{tabular}{|c|c|c|}
\hline Surface sand and clay & 96 & 96 \\
\hline Coarse sand and clay & 18 & 114 \\
\hline Coarse sand & 12 & 126 \\
\hline Medium sand and clay & 16 & 142 \\
\hline Coarse sand & 14 & 156 \\
\hline Coarse sand and clay .. & 12 & 168 \\
\hline Gravel and small boulders & 9 & 177 \\
\hline Coarse sand and clay -- & 16 & 193 \\
\hline n-n & 10 & 203 \\
\hline Gravel and clay ....... & 14 & 217 \\
\hline Medium sand and gravel & 11 & 228 \\
\hline Gravel and clay ........ & 13 & 241 \\
\hline Gravel & 8 & 249 \\
\hline Clay and gravel ........... & 19 & 268 \\
\hline Coarse sand ............ & 6 & 274 \\
\hline Clay and gravel .............. & 22 & 296 \\
\hline Medium sand and gravel & 13 & 309 \\
\hline Coarse sand and gravel & 14 & 323 \\
\hline Clay and coarse gravel & 15 & 338 \\
\hline Coarse gravel, coarse sand & 13 & 351 \\
\hline Gravel with clay & 17 & 368 \\
\hline Coarse gravel & 6 & 374 \\
\hline Hard sand and clay & 12 & 386 \\
\hline Coarse gravel and clay & 9 & 395 \\
\hline Hard sand, coarse gravel & 10 & 405 \\
\hline
\end{tabular}


11/9-34K. Millhollin. Altitude about 2,300 feet. Drilled by Pauley Bros. in April 1950. 6-incin casing.

\begin{tabular}{|c|c|}
\hline Material & $\begin{array}{l}\text { Thickness } \\
\text { (feet) }\end{array}$ \\
\hline
\end{tabular}

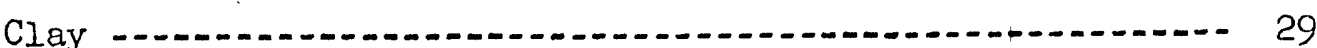

Coarse gravel, water -........... 130

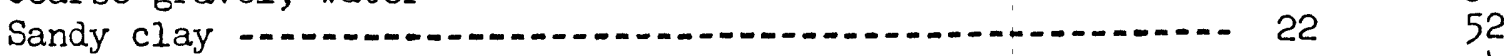

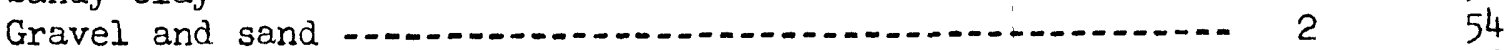

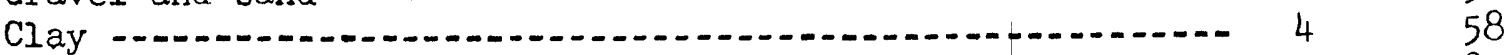

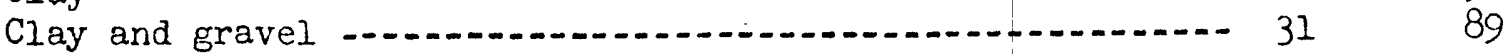

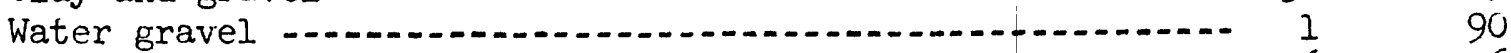

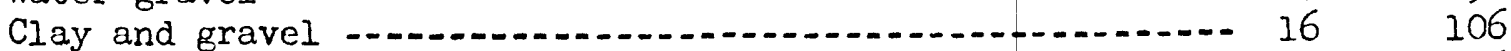

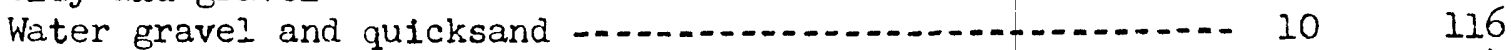

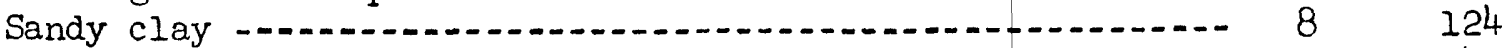

Sandy clay, some streaks of coarse gravel and sand ...... $23 \quad 147$

11/9-36Al. U. S. Borax and Chemical Corp., well 28. Altitude 2,323.6 feet. Drilled by Roscoe Moss Drilling Co. in June 1955. 16-inch casing zero to 486 feet; perforated 110 to 470 feet; uncased inle 486 to 610 feet.

Sandy clay ........................................ 32

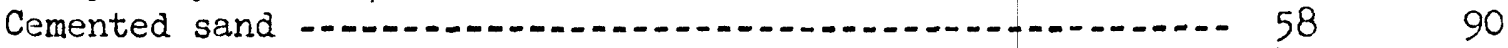

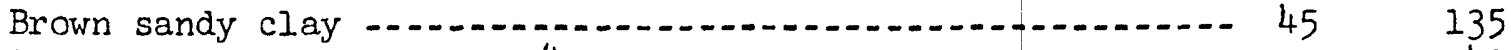

Coarse sand and gravel, 1/4-inch .................. 7 142

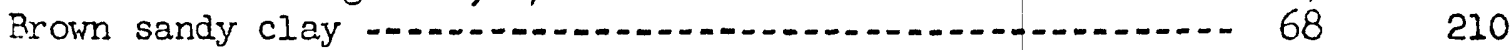

ioulders in cemented sand ....... 120

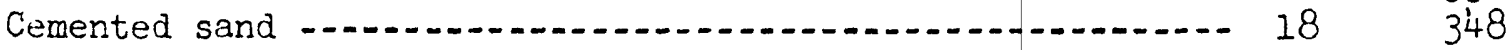

Eoulders of sandy clay ........................ $27 \quad 375$

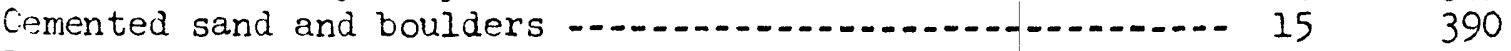

Fulders and sandy clay ............................... 595

Sindy clay and boulders, some shale .................. $151 \quad 546$

Sindy clay and boulders ................................ 64

11/9-36Cl. U. S. Borax and Chemical Corp., well 30. Altitude about 3,325 feet. Drilled by Reliance Drilling Co. in June 1955. 12-inch casing zero to 407 feet; perforated 96 to 407 feet.

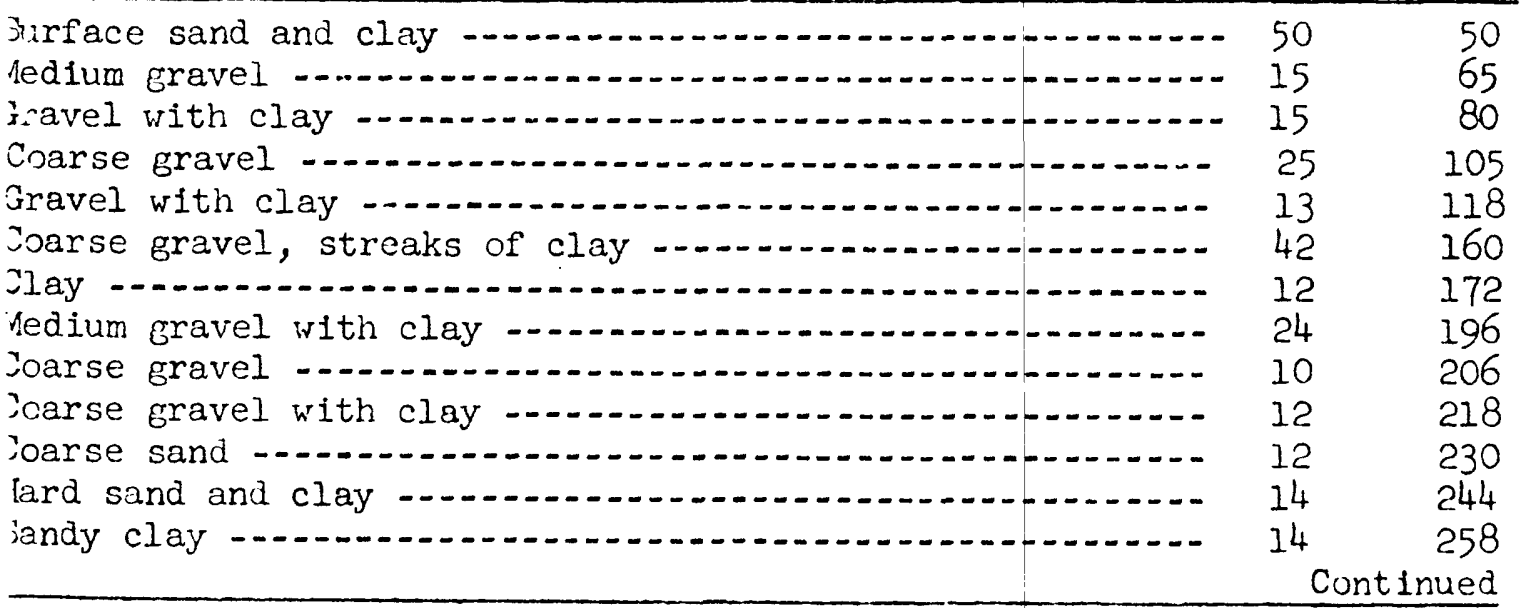


$11 / 9-36 \mathrm{Cl}$.--Cont Inued

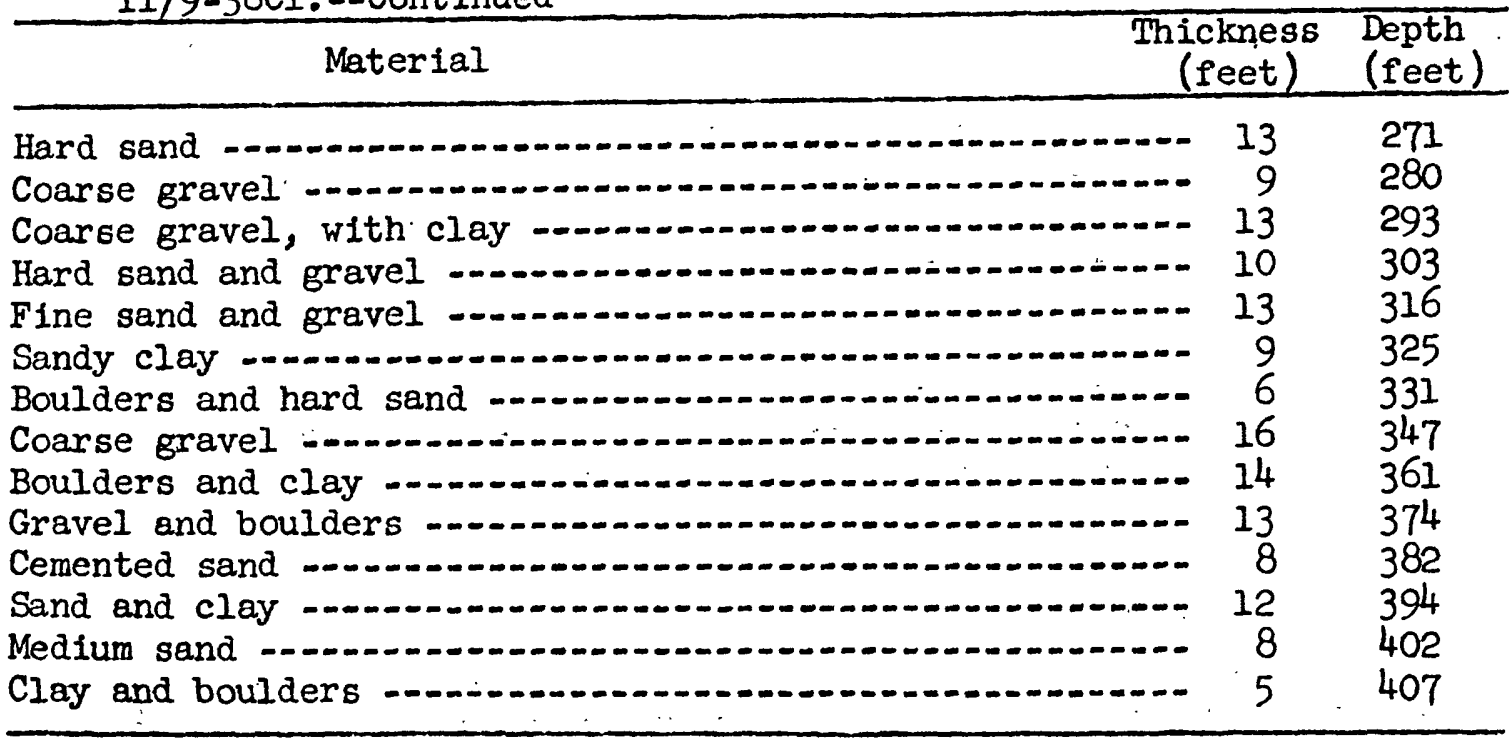

11/9-36DI. U. S. Borax and Chemical Corp., well 29. Altitude about 2,320 feet. Drilled by. Roscoe Moss Drilling Co. in June 1955. 14-inch casing zero to 370 feet; perforated 110 to 350 feet; uncased hole 370 to 414 feet.

Sand -...............

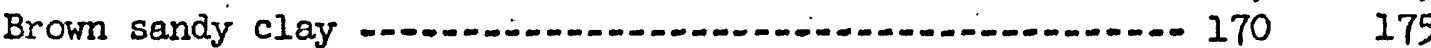

Sandy clay, very little clay ......................... $50 \quad 225$

Sandy clay and gravel, $1 \frac{1}{2}$ inches

Cemented sand and gravel, 2 inches .................. $5 \quad 340$

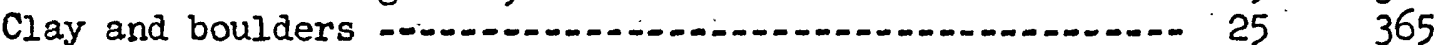

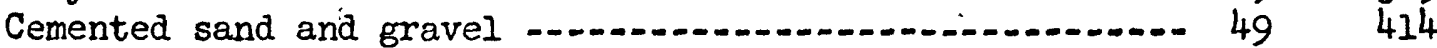

11/9-36HI. U. S. Borax and Chemical Corp., well 31. Altitude about 2,325 feet, Drilled by Reliance Drilling Co. in July 1955. 12-inch casing zero to 250 feet; perforated 96 to 250 feet; uncased hole 250 to 300 feet.

Surface sand and clay Hard coarse sand Gravel and clay

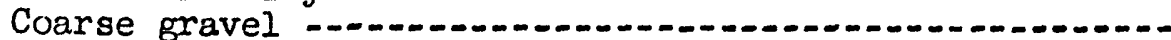
Gravel with clay

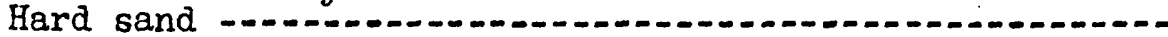
Small gravel and clay

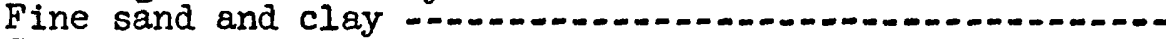

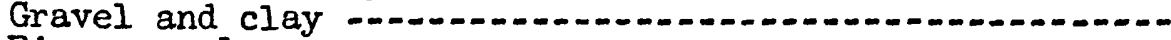

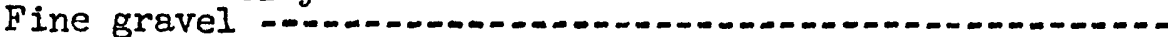

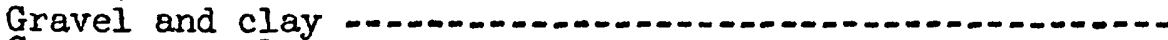

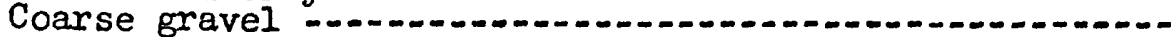

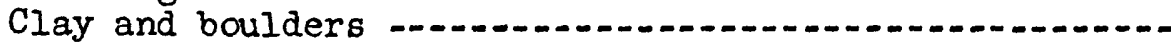
Cemented sand and boulders ....................................... Clay and boulders Gravel and clay -...t.t. Clay and boulders

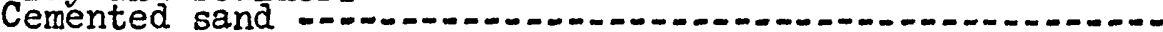
Cemented sand and rock

$\begin{array}{ll}50 & 50 \\ 11 & 61 \\ 17 & 78 \\ 14 & 92 \\ 12 & 100 \\ 12 & 116 \\ 10 & 126 \\ 14 & 140 \\ 10 & 150 \\ 19 & 169 \\ 5 & 174 \\ 7 & 181 \\ 33 & 214 \\ 5 & 219 \\ 11 & 230 \\ 11 & 241 \\ 19 & 250 \\ 13 & 263 \\ 37 & 300\end{array}$


11/9-36R1. U. S. Air Force. Altitude about 2,315 feet. Drilled by Mogle Bros. Drilling Co. In April 1953. 10-inch casing zero to 298 feet, perforated 100 to 132 feet.

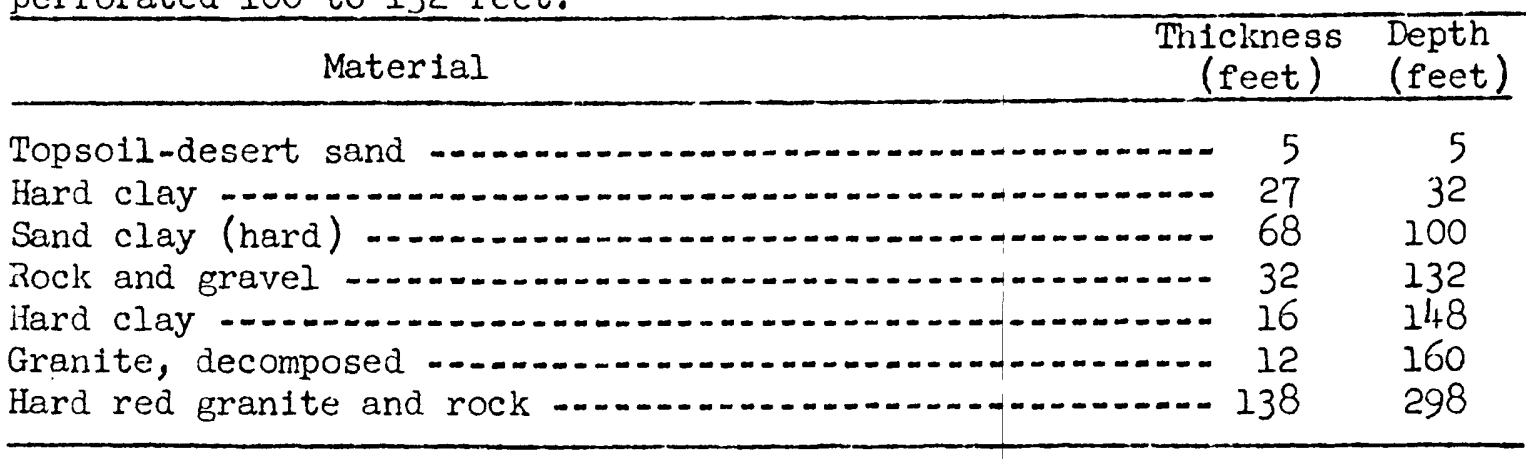

11/10-36Al. A. F. Green. Altitude about 2,340 feet. Drilled by D. W. Slocum in June 1957. 10-inch casing zero to 300 feet; perforated 198 to 300 feet.

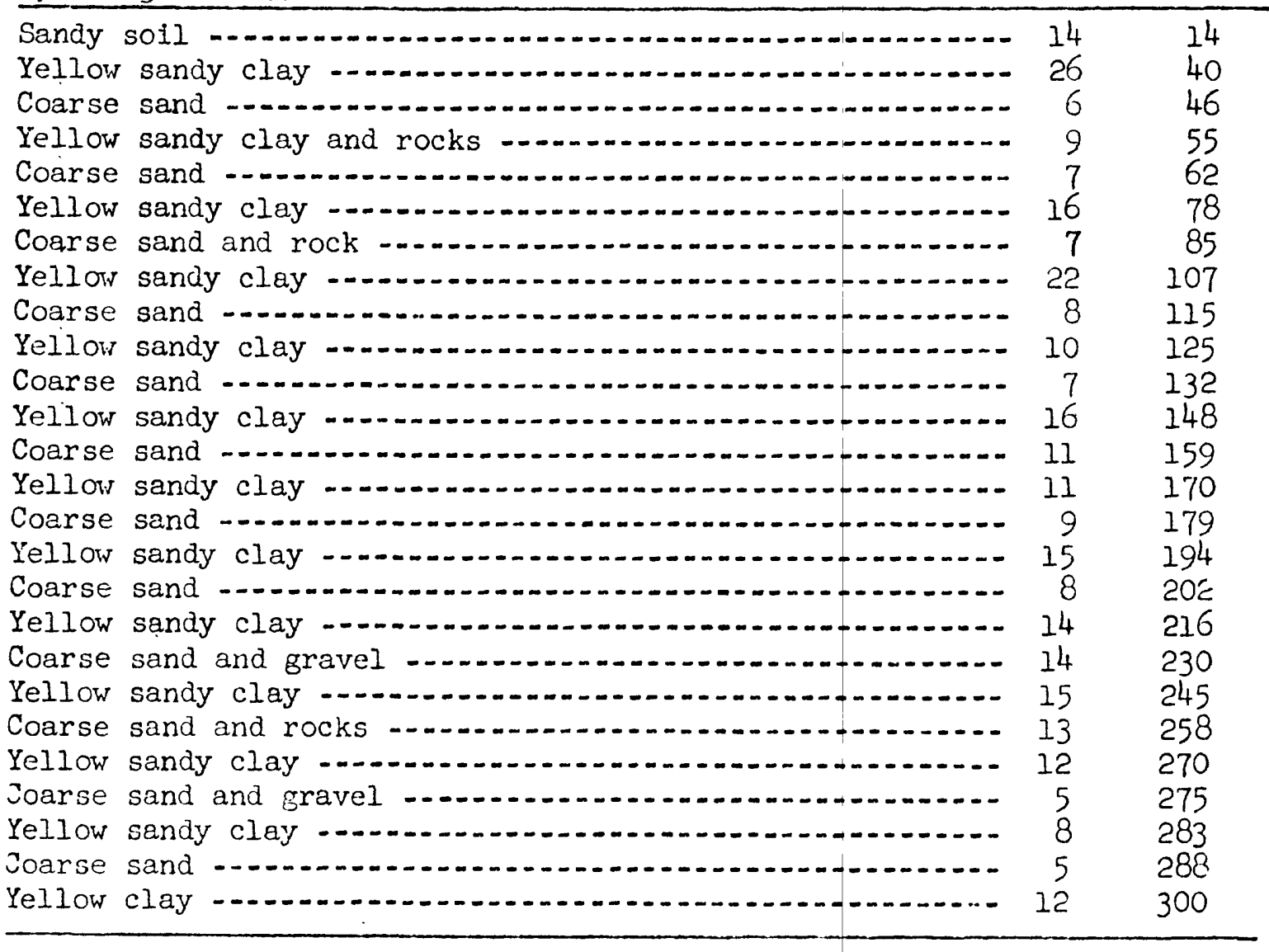


1I/10-36Bl. A. F. Green. Alt1tude about 2,345 feet. Drilled by D. W. Slocum. 8-inch casing zero to 238 feet; perforated 159 to 238 feet.

\begin{tabular}{|c|c|c|}
\hline Materlal & $\begin{array}{c}\text { Thickness } \\
\text { (feet) }\end{array}$ & $\begin{array}{l}\text { Depth } \\
\text { (feet) }\end{array}$ \\
\hline Fine sand and brown clay & 18 & 18 \\
\hline Clay & $\begin{array}{r}28 \\
5\end{array}$ & $\begin{array}{l}46 \\
51\end{array}$ \\
\hline $\begin{array}{l}\text { Sand } \\
\text { Clay and gravel }\end{array}$ & 32 & 83 \\
\hline Sand & 15 & 98 \\
\hline Gray clay & 24 & 112 \\
\hline Hard white clay & 17 & 129 \\
\hline Clay with hard streaks ... & 11 & 140 \\
\hline Hard clay, small rocks. & 11 & 151 \\
\hline Good water sand & 8 & 159 \\
\hline Clay, gravel mixed & 9 & 168 \\
\hline Sand fine & 8 & 176 \\
\hline Clay & 14 & 190 \\
\hline Gravel ..... & 14 & 204 \\
\hline Hard packer clay & 14 & 218 \\
\hline Sand gravel & 20 & 238 \\
\hline Rock - & -- & 238 \\
\hline
\end{tabular}

11/10-36Hl. Atchison, Topeka, and Santa Fe Ra1lway. Altitude 2,337.0 feet. Drilled by Beylic Bros. Drilling Co, in 1953. 10-inch casing zero to 300 feet; uncased hole 300 to 320 feet.

\begin{tabular}{|c|c|c|}
\hline Topsoil & 5 & 5 \\
\hline Sand -100 & 5 & 10 \\
\hline Sand and clay & 10 & 20 \\
\hline Sandy clay & 30 & 50 \\
\hline Fine sand, gravel and clay, hard ...... & 10 & 60 \\
\hline Coarse gravel and sand, hard & 20 & 80 \\
\hline Coarse gravel and clay & 10 & 90 \\
\hline Soft clay and gravel & 10 & 100 \\
\hline Coarse gravel and hard clay .. & 10 & 110 \\
\hline Soft clay and gravel & 10 & 120 \\
\hline Sandy clay and gravel & 20 & 140 \\
\hline Coarse sand and clay & 10 & 150 \\
\hline Coarse sand, gravel and clay & 50 & 200 \\
\hline Coarse gravel and clay; hard & 10 & 210 \\
\hline Small boulders, gravel and clay & 10 & 220 \\
\hline Sand and gravel & 10 & 230 \\
\hline Sand, gravel and clay, hard & 20 & 250 \\
\hline Gravel, sand, and hard clay & 10 & 260 \\
\hline Sand, gravel, and hard clay & 20 & 280 \\
\hline Decomposed granite & 35 & 315 \\
\hline 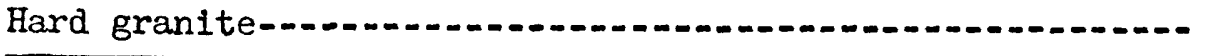 & 5 & 320 \\
\hline
\end{tabular}


29/39-33H. Stocton, Works, et al. Altitude about 2,105 feet. Drilled by D. W. Slocum in July 1953. 16-inch casing zero to 460 feet; perforated 200 to 460 feet.

\begin{tabular}{|c|c|c|}
\hline Material & $\begin{array}{c}\text { Thickness } \\
\text { (feet) }\end{array}$ & $\begin{array}{l}\text { Depth } \\
\text { (feet) }\end{array}$ \\
\hline 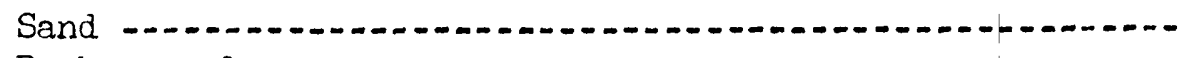 & 6 & 6 \\
\hline 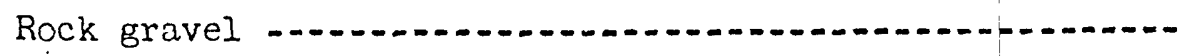 & 90 & 96 \\
\hline Yellow clay & 8 & 104 \\
\hline Rock gravel & 15 & 119 \\
\hline Yellow clay rock & 45 & 164 \\
\hline Gravel rock & 22 & 186 \\
\hline Yellow clay & 32 & 218 \\
\hline Coarse gravel rock & 34 & 252 \\
\hline Yellow clay rock & 24 & 276 \\
\hline Coarse gravel rock & 14 & 290 \\
\hline Yellow clay rock & 16 & 306 \\
\hline Cọarse gravel rock & 14 & 320 \\
\hline Yellow clay rock & 24 & 344 \\
\hline Coarse sand gravel & 6 & 350 \\
\hline Yellow clay rock & 45 & 395 \\
\hline Coarse sand gravel ... & 11 & 406 \\
\hline Clay rock & 34 & 440 \\
\hline Rock brown sand & 10 & 450 \\
\hline rlay rock & 6 & 456 \\
\hline Rock & 4 & 460 \\
\hline
\end{tabular}

29/39-33KL. Jesse Stocton. Alt1tude about 2,070 feet. Drilled by D. W. Slocum in June 1956. 16-inch casing zero to 402 feet; perforated 210 to 402 feet.

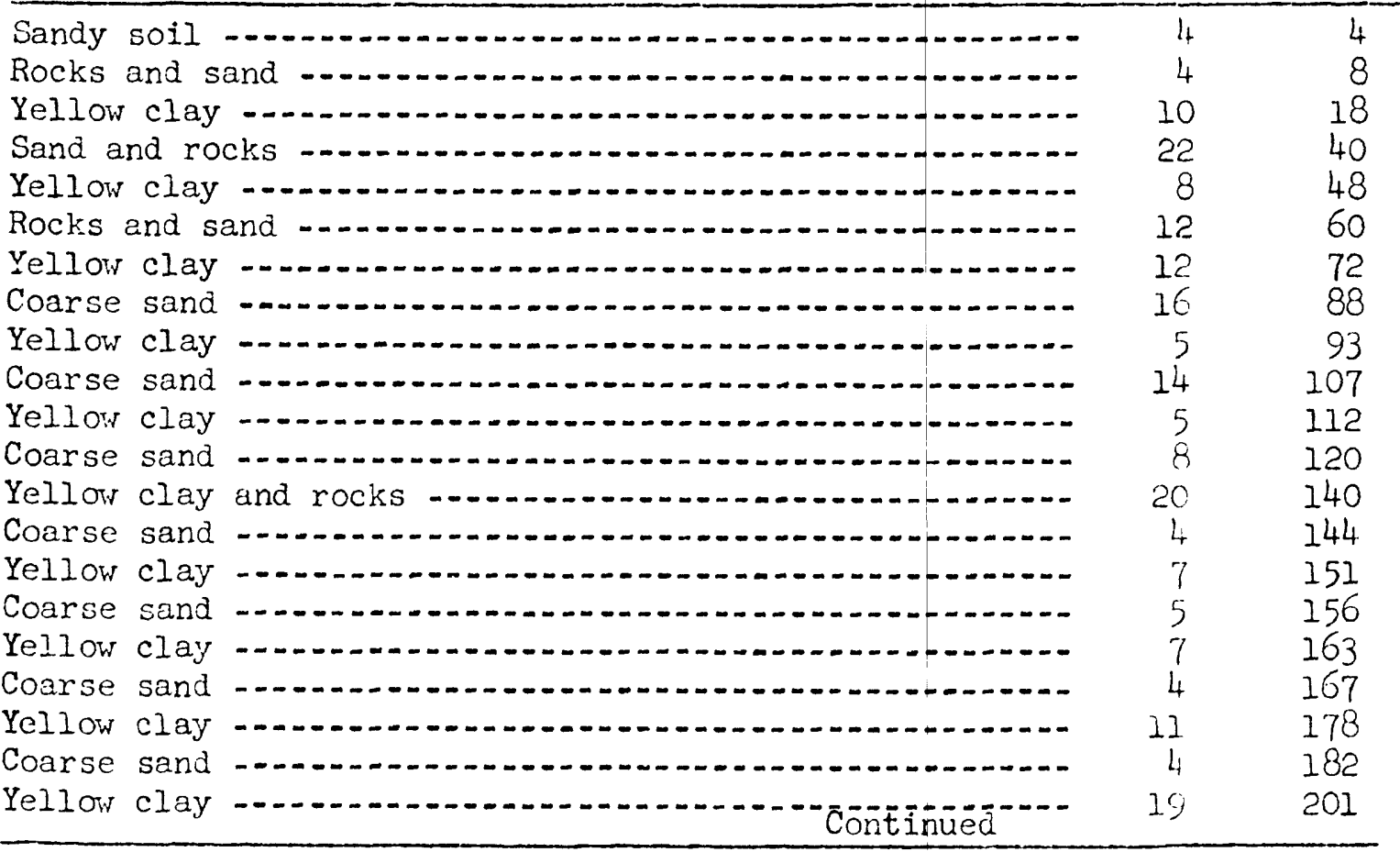


29/39-33K..--Continued

\begin{tabular}{|c|c|c|c|}
\hline & Material & $\begin{array}{l}\text { kness } \\
\text { eet) }\end{array}$ & $\begin{array}{l}\text { Depth } \\
\text { (feet) }\end{array}$ \\
\hline Coarse sand & 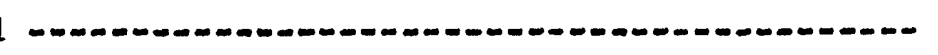 & 2 & 203 \\
\hline Yellow clay & 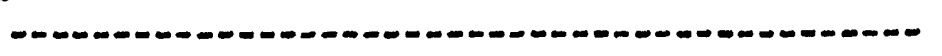 & 6 & 209 \\
\hline Coarse sand & - & 5 & 214 \\
\hline Yellow clay & 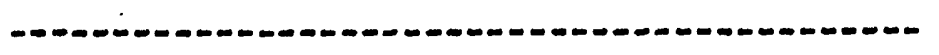 & 14 & 228 \\
\hline Coarse sand & - & 6 & 234 \\
\hline Yellow clay & 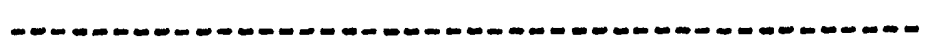 & 11 & 245 \\
\hline Coarse sand & - & 7 & 252 \\
\hline Yellow clay & 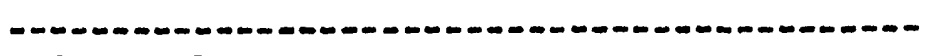 & 12 & 264 \\
\hline Coarse sand & 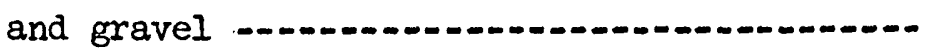 & 8 & 272 \\
\hline Yellow clay & 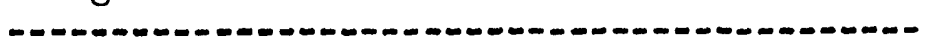 & 8 & 280 \\
\hline Coarse sand & and gravel & 8 & 288 \\
\hline Yellow clay & - n- & 6 & 294 \\
\hline Coarse sand & and gravel & 6 & 300 \\
\hline Yellow clay & 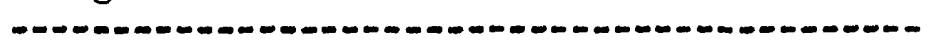 & 11 & 311 \\
\hline Coarse sand & 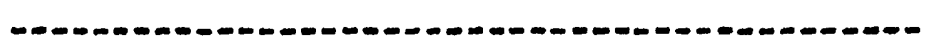 & 7 & 318 \\
\hline Yellow clay & $-\cdots-\infty-\infty-\infty-\infty-\infty \cdots$ & 11 & 329 \\
\hline Coarse sand & and gravel ......... & 6 & 335 \\
\hline Yellow clay & 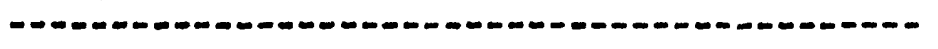 & 9 & 344 \\
\hline Coarse sand & and gravel & 4 & 348 \\
\hline Yellow clay & - & 12 & 360 \\
\hline Coarse sand & and gravel & 4 & 364 \\
\hline Yellow clay & 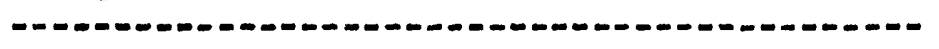 & 12 & 376 \\
\hline Coarse sand & and gravel & 5 & 381 \\
\hline Yellow clay & -am-and & 21 & 402 \\
\hline
\end{tabular}

30/37-13E1. Crookshank, well 1. Altitude about 2,200 feet. 18-inch surface casing, not perforated.

Sand and gravel

Hard sand

Clay, hard sand streaks

Shale and sand streaks

Shale

Gravel, small amount of water

Hard sand -

Brown shale -............

Hard sand and shale streaks

Shale and hard shells

Sandy shale (2...

Brown sandy shale (.......

Sand and shale streaks

Shale -

Sandy shale -

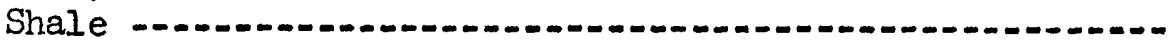

Sand -...

Streaks, shale and sand

Blue shale

Blue shale and sand streaks

\begin{tabular}{rr}
103 & 103 \\
70 & 173 \\
157 & 330 \\
245 & 575 \\
25 & 600 \\
15 & 615 \\
20 & 635 \\
50 & 685 \\
87 & 772 \\
118 & 890 \\
70 & 960 \\
157 & 1,117 \\
183 & 1,300 \\
15 & 1,315 \\
10 & 1,325 \\
32 & 1,357 \\
23 & 1,380 \\
110 & 1,490 \\
42 & 1,532 \\
65 & 1,597 \\
Cont1nued \\
\hline
\end{tabular}


30/37-13E1.--Continued

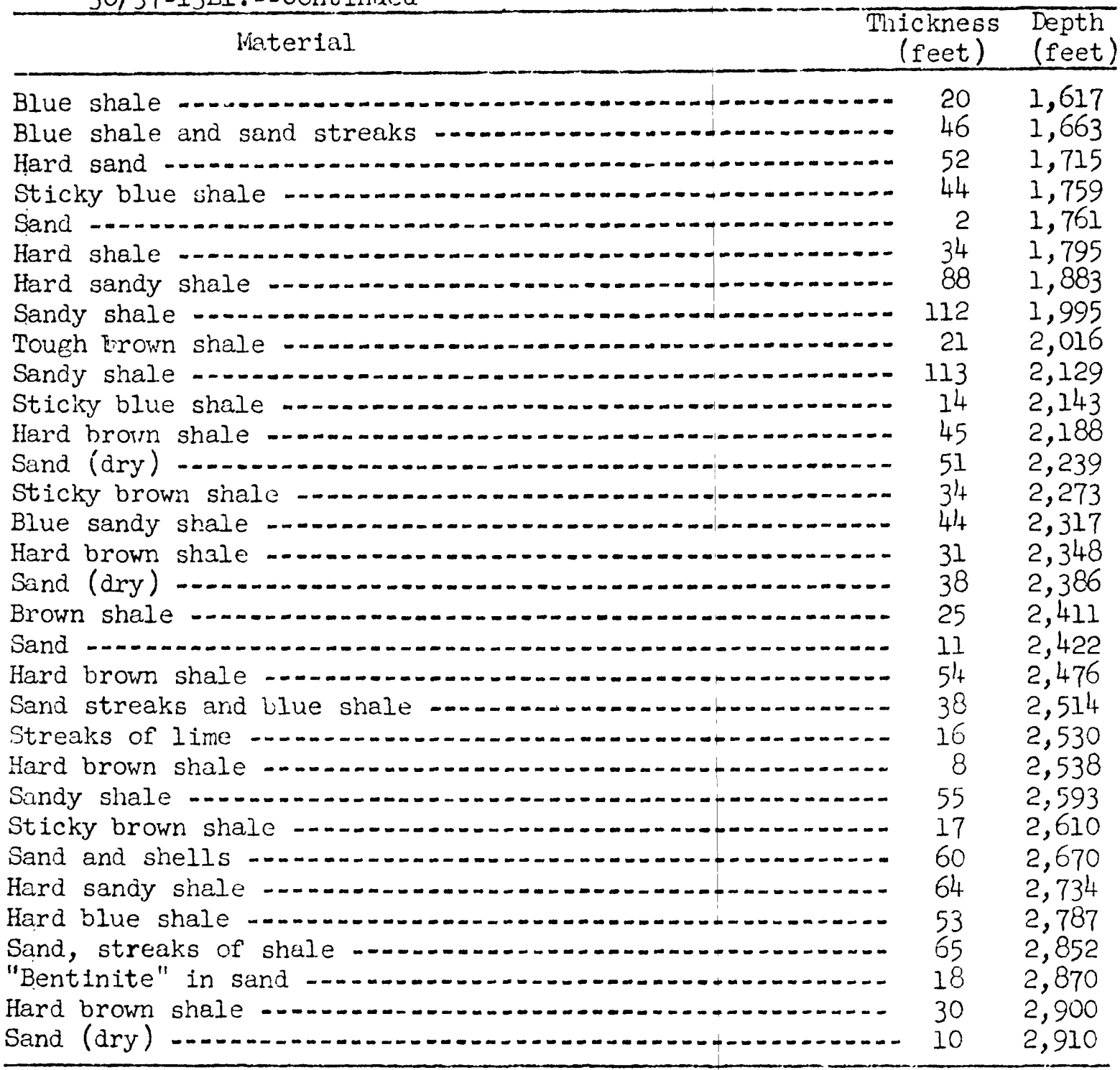

30/37-13Fl. Crookshank, well 1A. Altitude about 2,100 feet. 12 -inch surface casing, not perforated.

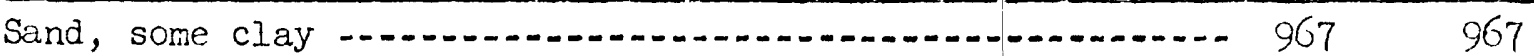

Sand, light buff-yellow color, fine- to medium-grained,

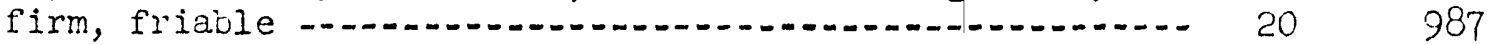

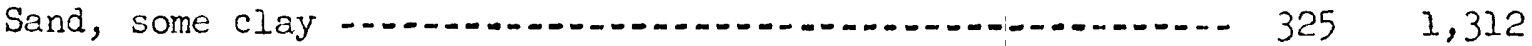

Claystone or mudstone, light greenish-brown or buff, massive, no apparent dips; and fine to medium sand with interstitial. silt, greenish-gray _.......... 20 1,332

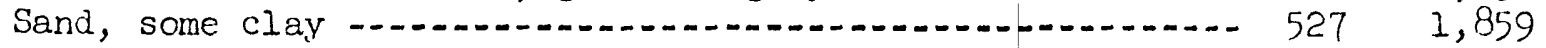


30/37-24Bl. Southern Pacific Co. Altitude about 2,010 feet. 12-1nch casing.

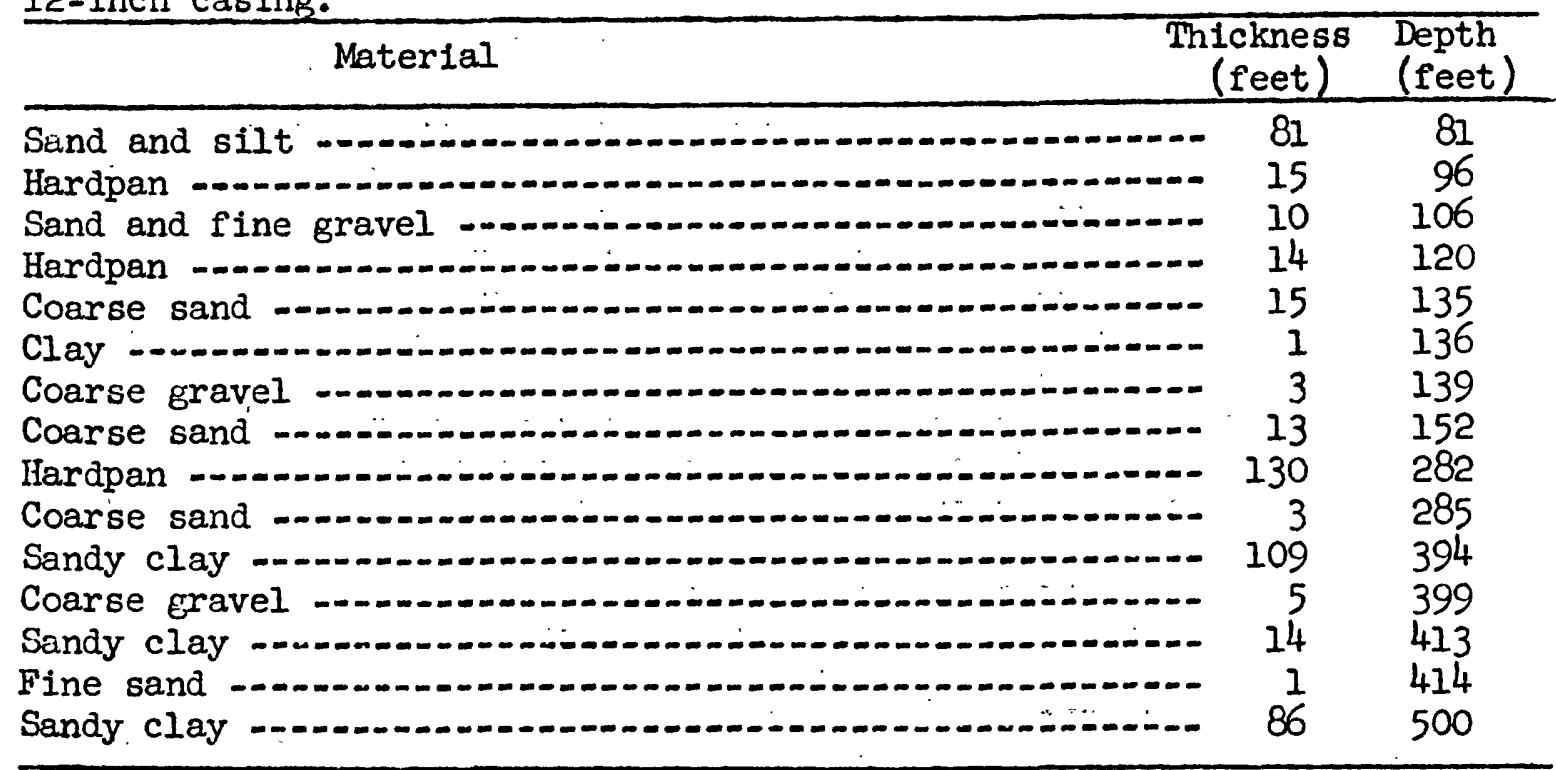

30/37-25M?. M and $R$, Cantil Ranch, well 4. Altitude about 1,975 feet. 18-inch casing zero to 282 feet, 12-inch casing 288-692 feet; perforated $120-282$ and 238-69? feet.

Sand
Sandy clay
Sand
Coarse sand $\ldots$

30/37-26EI. M and $R$, Cantil Rancis, well 13. Altitude about 2,040 feet. Drilled by Clarence Raley in 1950. 14-inch casing zero to 485 feet, perforated 233 to 485 feet.

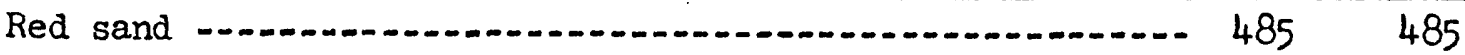


30/37-35DI. $M$ and $R$, Cantil Ranch, well 5. Altitude about 2,020 feet. 18-inch casing zero to 282 feet, 12-1nch casing 282 to 844 feet; perforated 120 to 282 and 288 to 844 feet.

\begin{tabular}{|c|c|c|}
\hline Material & $\begin{array}{l}\text { kness } \\
\text { eet.) }\end{array}$ & $\begin{array}{l}\text { Depth } \\
\text { (feet) }\end{array}$ \\
\hline Topsoil & 20 & 20 \\
\hline Sand & 30 & 50 \\
\hline Boulders and clay & 40 & 90 \\
\hline Sand & 25 & 115 \\
\hline Blue clay & 5 & 120 \\
\hline Sand, blue clay & 60 & 180 \\
\hline Gray sand & 32 & 21.2 \\
\hline Sand with streaks of clay & 103 & 315 \\
\hline Sand & 56 & 371 \\
\hline Sand with streaks of clay & 84 & 455 \\
\hline Sandy clay & 40 & 495 \\
\hline Sand & 65 & 560 \\
\hline Sand with strealss of clay & 64 & 624 \\
\hline Sandy clay & 56 & 680 \\
\hline Hard sand & 22 & 702 \\
\hline Fine sand & 32 & 734 \\
\hline Sand with streaks of clay & 90 & 824 \\
\hline Clay & 20 & 844 \\
\hline
\end{tabular}

30/37-35Ql. $M$ and $R$, Cantil Ranch, well 9. Altitude about 2,030 feet. 20-inch casing zero to 408 feet, 12 -inch casing 408 to 310 feet; perforated 246 to 408 and 414 to 810 feet.

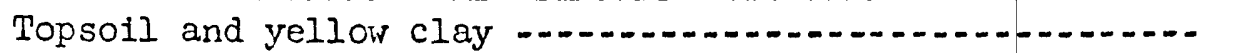

Blue clay

Blue sandy clay

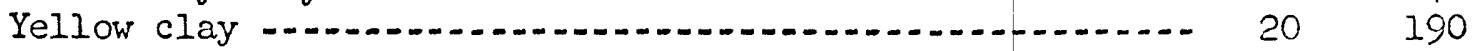

Blue sand with streaks of clay .................... $140 \quad 330$

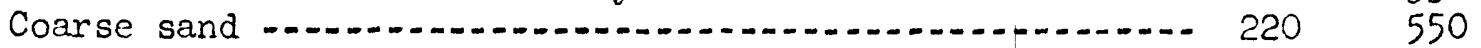

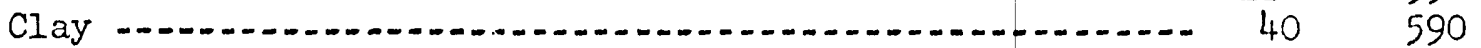

Coarse sand and streaks of clay ................... $120 \quad 710$

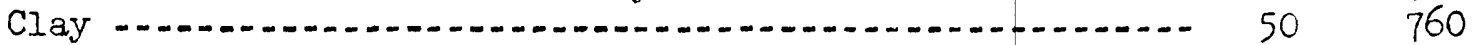

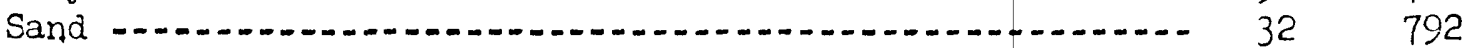

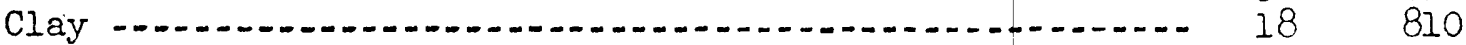

30/37-36Cl. M and R, Cantil Rinch, well 14. Altitude about 2,000 feet. 14-1nch casing zero to 500 feet. perforated 248 to 500 feet.

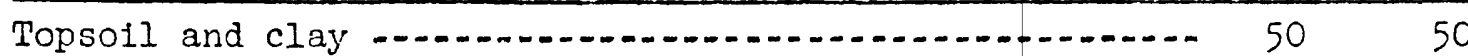

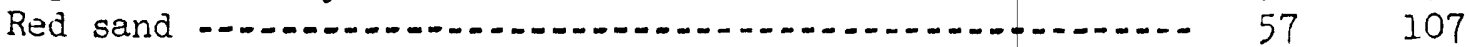

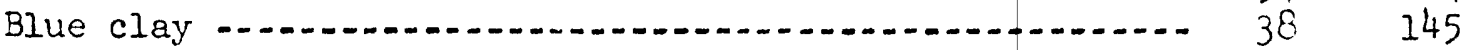

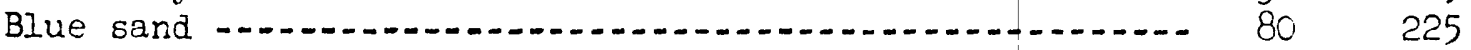

Sand with streaks of clay ....................... $115 \quad 340$

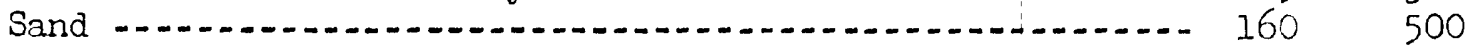


30/37-36Gl. M and R, Cantil Ranch, well 7. Altitude 1,981.0 feet. Drilled by S. F. Caty. 14-inch casing; perforated Interval: 12-52, $106-110,138-144,171-180,238-250,293-309,418-424,436-440,446-450$, $457-463,767-787,816-824,832-838,902-907,916-919$ feet.

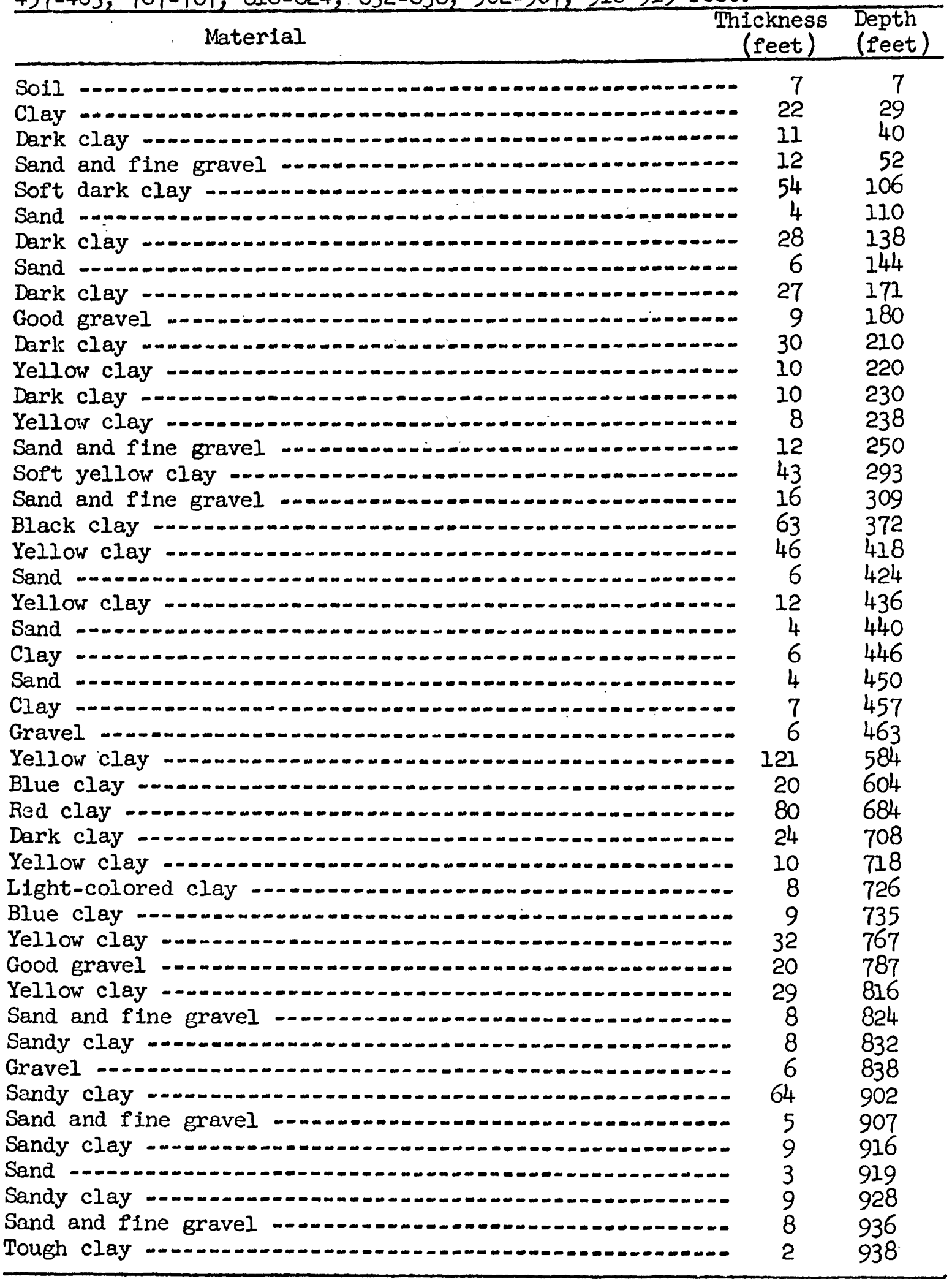


30/37-36Kl. M and $R$, Cantil Ranch, well 15. Altitude about 2,005 feet. 14-inch casing zero to 527 feet, perforated 275 to 527 feet.

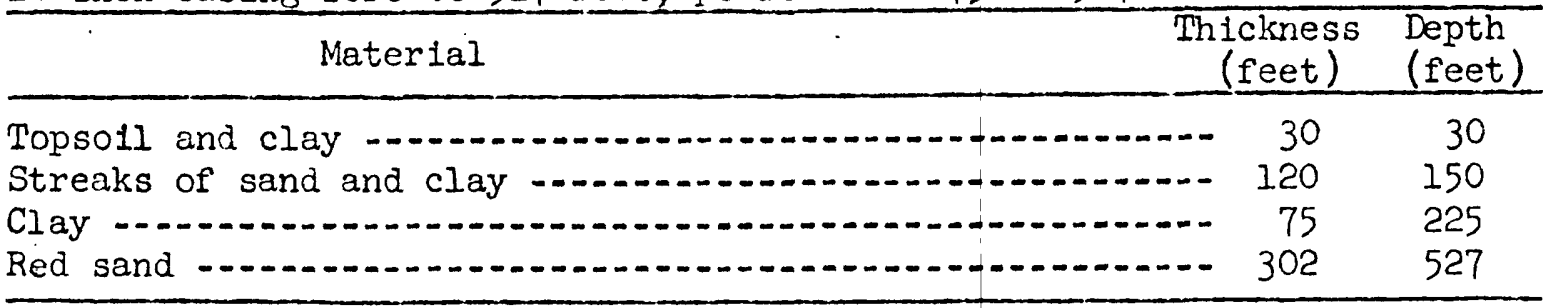

30/37-36Nl. $M$ and $R$, Cantil Ranch, well 10. Altitude about 2,025 feet. 20-inch casing zero to 410 feet, 12-inrh casing 410 to 590 feet; perforated 244 to 410 and 416 to 590 feet.

\begin{tabular}{|c|c|c|}
\hline Topsoil and clay & 60 & 60 \\
\hline Red sand & 60 & 120 \\
\hline Clay & 10 & 130 \\
\hline Coarse sand and boulders ............ & 30 & 160 \\
\hline Sand with thin streaks of clay ... & 120 & 280 \\
\hline 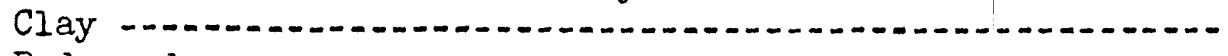 & 40 & 320 \\
\hline Red sand & 190 & 510 \\
\hline Sendy clay & 25 & 535 \\
\hline 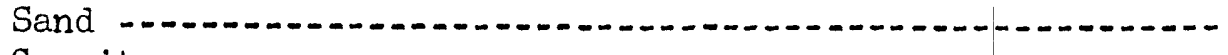 & 45 & 580 \\
\hline Granite & 10 & 590 \\
\hline
\end{tabular}

30/38-19Al, J. E. Sprott, formerly Crookshank. Altitude about 1,950 feet. Uncased.

\begin{tabular}{|c|c|c|}
\hline Surface sand & 72 & 72 \\
\hline Sand and gravel & 40 & 112 \\
\hline Sticky blue shale & 13 & 125 \\
\hline Sand with hard streaks & 31 & 156 \\
\hline 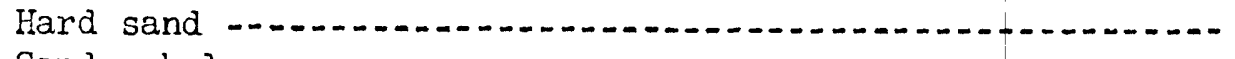 & 9 & 165 \\
\hline Sandy shale & 30 & 195 \\
\hline Sandy blue shale & 15 & 210 \\
\hline Sand with streaks of hard sand & 50 & 260 \\
\hline 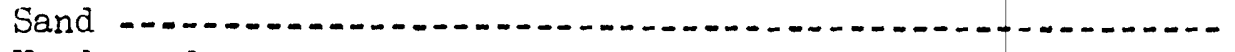 & 20 & 280 \\
\hline Hard sand $\cdots+\cdots$ & 5 & 285 \\
\hline Sticky clay & 5 & 290 \\
\hline Sand & 40 & 330 \\
\hline Sticky blue shale & 10 & 340 \\
\hline Sand and gravel & 20 & 360 \\
\hline 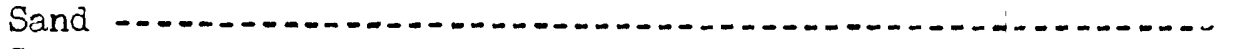 & 40 & 400 \\
\hline Clay and streaks of sand & 40 & 440 \\
\hline Sand and streaks of clay & 10 & 450 \\
\hline Sand and boulders & 50 & 500 \\
\hline Sandy shale & 40 & 540 \\
\hline Hard sand and streaks of clay & 40 & 580 \\
\hline Hard sand $-1,-1$ & 20 & 600 \\
\hline Hard sand and streaks of gypsum & 40 & 640 \\
\hline Hard sand & 20 & 660 \\
\hline Sticky blue shale streaked with sand & 45 & 705 \\
\hline Shale streaked with hard sand & $\begin{array}{l}35 \\
\text { Cor }\end{array}$ & $\begin{array}{l}740 \\
\text { sed }\end{array}$ \\
\hline
\end{tabular}


30/38-19A1..-Cont Inued

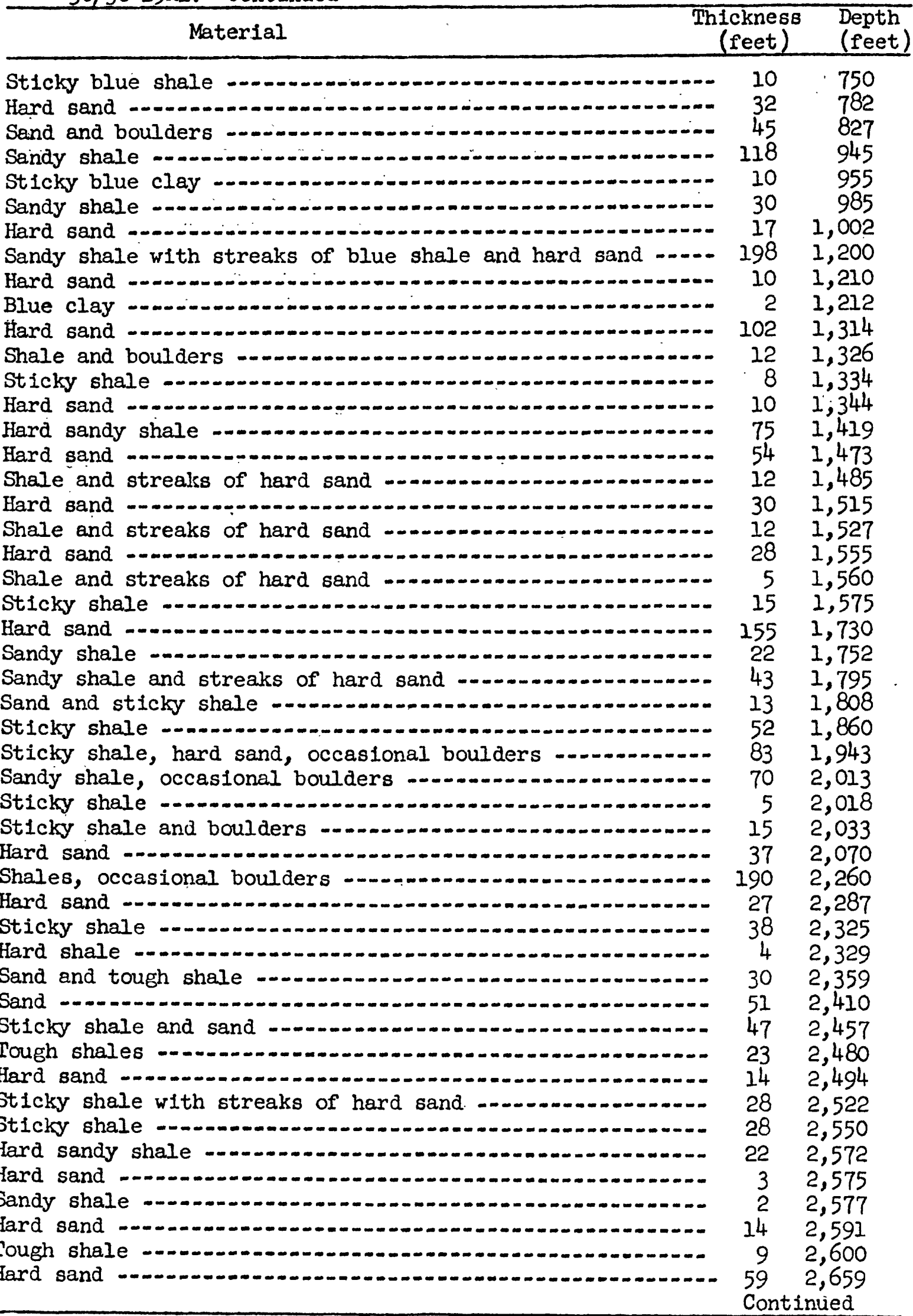


30/38-19A1. ---Cont Inued

\begin{tabular}{|c|c|c|}
\hline & $\begin{array}{l}\text { Thickness } \\
\text { (feet) }\end{array}$ & $\begin{array}{l}\text { Depth } \\
\text { (feet) }\end{array}$ \\
\hline Hard & sand and shale & 2,661 \\
\hline Tough & sand and shale $\ldots$ & 2,681 \\
\hline Shale & and streaks of hard sand & 2,691 \\
\hline Sandy & shale $\ldots \ldots-\ldots+25$ & 2,716 \\
\hline Sand, & dry & 2,727 \\
\hline Shale & 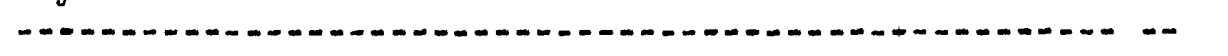 & 2,727 \\
\hline
\end{tabular}

30/38-19F1. Crookshank, well 2. Alt1tude about 1,970 feet. 18-inch surface casing.

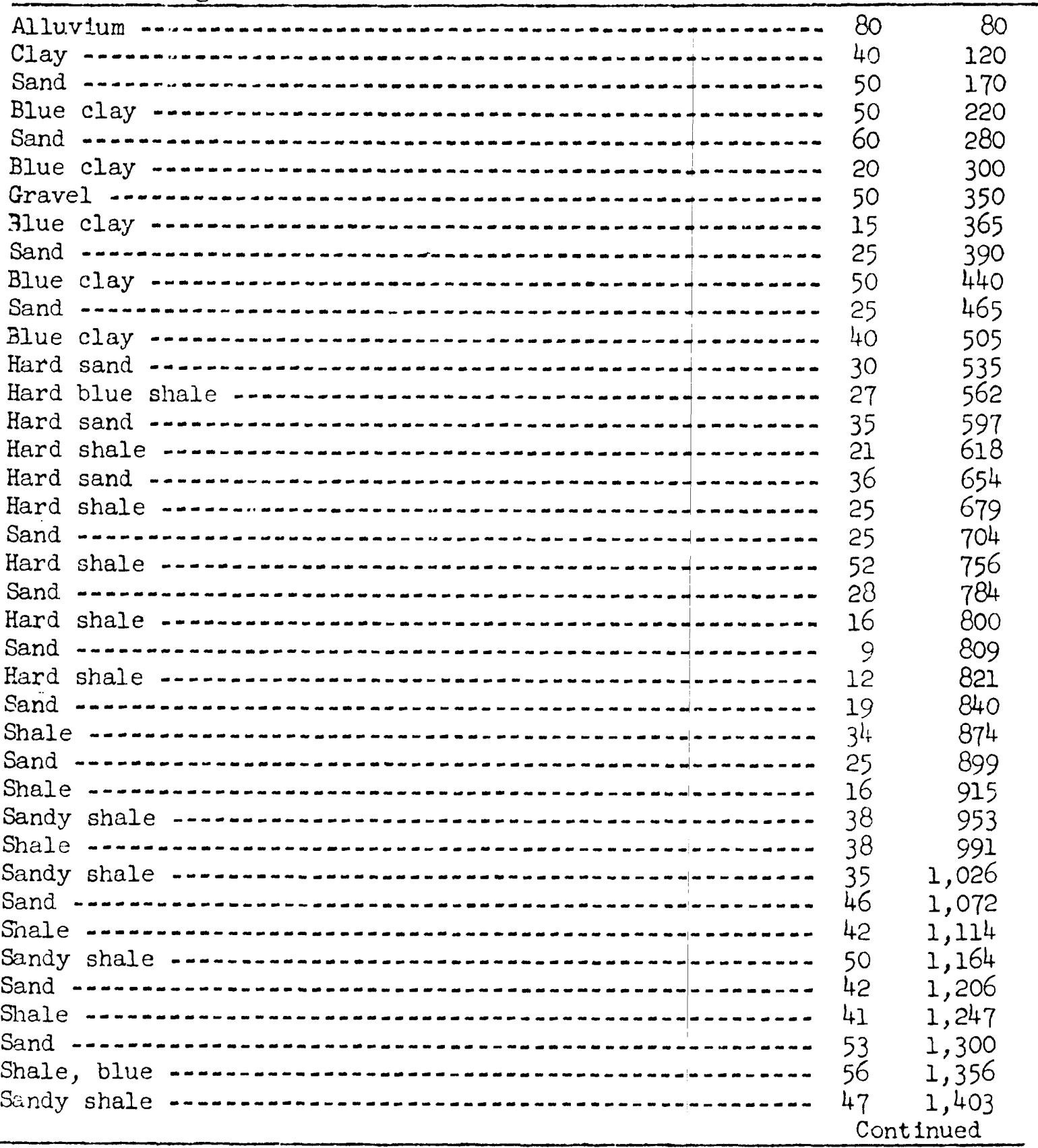


30/38-19F1.--Continued

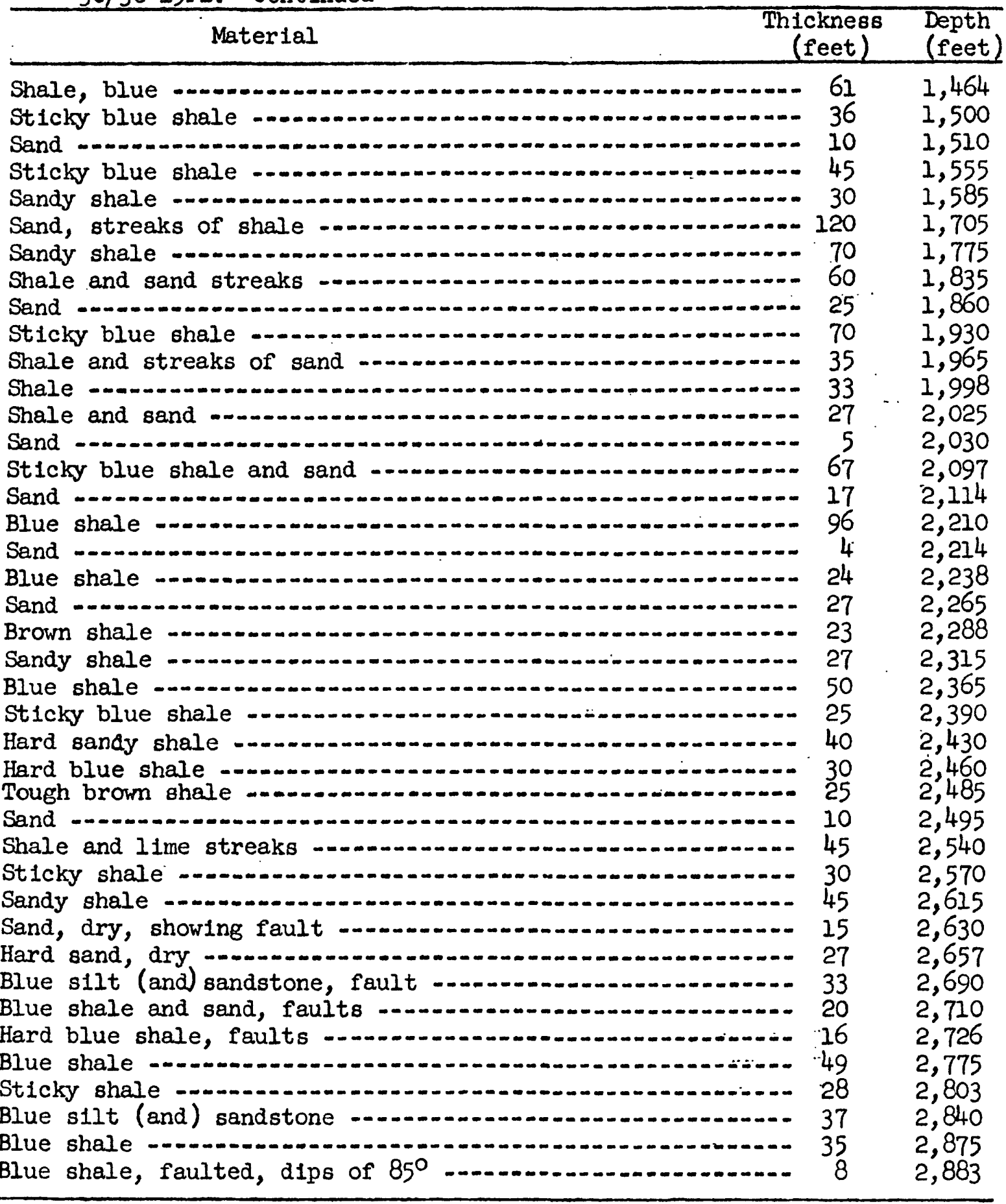


30/38-19PI. Crookshank, well 3. Alt1tude about 1,940 feet. $12-$ inch casing.

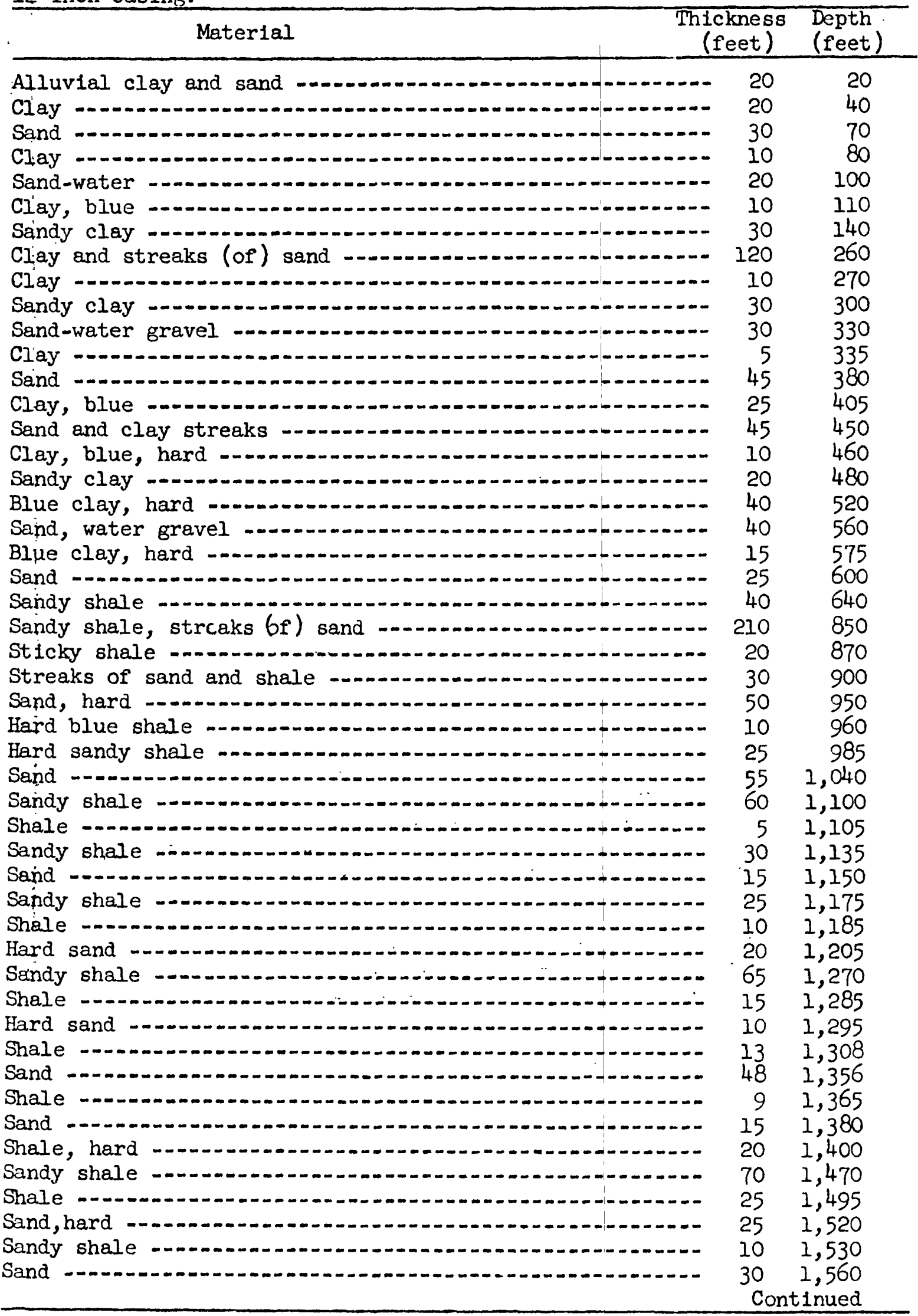


30/38-19P1 . --Continued

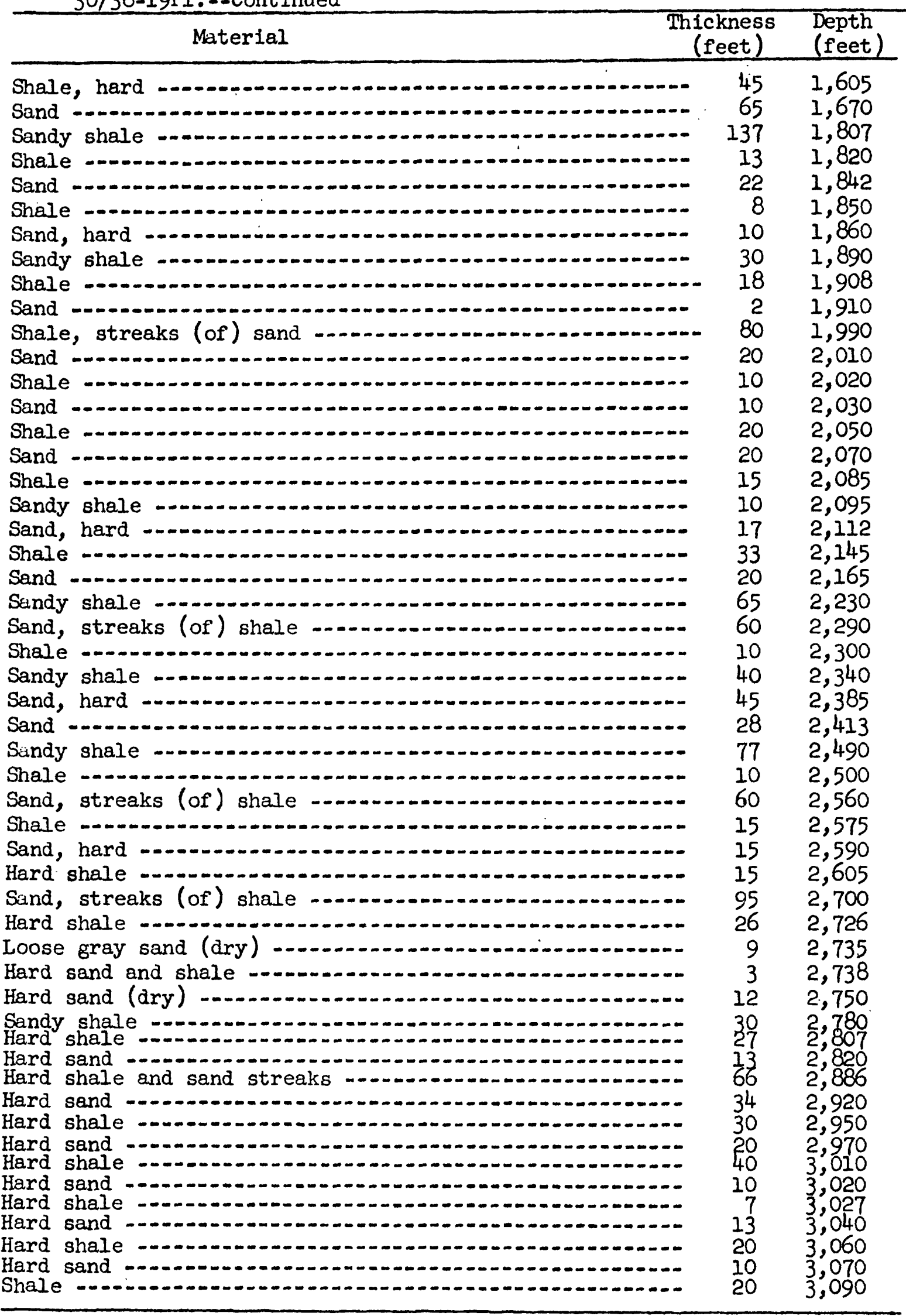


30/38-19P2. J: E. Sprott. Alt1tude about 1,940 feet. 18-inch casing.

\begin{tabular}{|c|c|c|c|c|c|}
\hline Material & $\begin{array}{l}\text { ickness } \\
\text { (feet) }\end{array}$ & $\begin{array}{l}\text { Depth } \\
\text { (feet) }\end{array}$ & Material & $\begin{array}{l}\text { nness } \\
\text { set) }\end{array}$ & $\begin{array}{l}\text { Depth } \\
\text { (feet) }\end{array}$ \\
\hline irface soll & 30 & 30 & Sand and streaks & & \\
\hline and and gravel, & & & of shale & 120 & 1,700 \\
\hline streaks of clay & -345 & 375 & Sandy shale & 75 & 1,775 \\
\hline Shale and boulders & -30 & 405 & Send and streaks of & & \\
\hline Tqugh shale and & & & shale & 80 & 1,855 \\
\hline $\begin{array}{l}\text { :streaks of sand } \\
\text { ough shale and sar }\end{array}$ & $\begin{array}{l}70 \\
-\quad 25\end{array}$ & $\begin{array}{l}475 \\
500\end{array}$ & Sticky blue shale -- & 75 & \\
\hline Sand and boulders & -90 & $\begin{array}{l}500 \\
590\end{array}$ & $\begin{array}{l}\text { Shale and streaks } \\
\text { of sand }\end{array}$ & 15 & 1,945 \\
\hline Sand and gravel --- & -15 & 605 & Shale & 50 & 1,995 \\
\hline Clay (and) boulders & 25 & 630 & Shale and sand -- & 30 & 2,025 \\
\hline Clay and gravel -.. & 58 & 688 & Sand & 2 & 2,027 \\
\hline Sand and gravel .- & -20 & 708 & Sticky blue sand & & \\
\hline Clay and gravel .- & -102 & 810 & with streaks of & & \\
\hline Clay and boulders & -10 & 820 & sand $\cdots$ & 63 & 2,090 \\
\hline Boulders & 5 & 825 & Sticky shale .... & 10 & 2,100 \\
\hline Boulders and tough & & & Sand & 14 & 2,114 \\
\hline clay & 12 & 837 & Shale & 68 & 2,182 \\
\hline Clay and boulders & 98 & 935 & Hard blue shale - & 28 & 2,210 \\
\hline Hard sand & 20 & 955 & Sand & 3 & 2,213 \\
\hline Hard sand and grave & -110 & 1,065 & Shale & 25 & 2,238 \\
\hline fard sand & -10 & 1,075 & Blue shale & 32 & 2,270 \\
\hline avel - - - & 45 & 1,120 & Brown shale & 11 & 2,281 \\
\hline Lose sand & 5 & 1,125 & Sandy and tough shale & 29 & 2,310 \\
\hline Hard sand and grav & 15 & 1,140 & Sandy shale & 30 & 2,340 \\
\hline lard send & 5 & 1,145 & Blue shale & 25 & 2,365 \\
\hline el & 45 & 1,190 & Sticky blue shale ... & 20 & 2,385 \\
\hline lough shale. . . & 10 & 1,200 & Hard sandy shale ...- & 5 & 2,390 \\
\hline icky shale & 10 & 1,210 & Sticky shale & 39 & 2,429 \\
\hline lay and streaks & & & StIcky blue shale -.- & 10 & 2,439 \\
\hline of sand & 35 & 1,245 & Hard blue shale & 25 & 2,464 \\
\hline sand and boulders- & 5 & 1,250 & Tough brown shale ... & 26 & 2,490 \\
\hline ad gravel --. & 42 & 1,292 & Hard sand & 5 & 2,495 \\
\hline sand and grave & 16 & 1,308 & Sticky shale with & & \\
\hline ind -5 & 4 & 1,312 & streaks of lime -.. & 20 & 2,515 \\
\hline 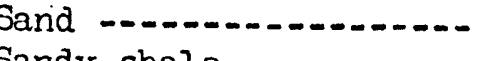 & $2^{2}$ & 1,314 & Brown shale with & & \\
\hline shale - - & 15 & 1,329 & streaks of lime ... & 25 & 2,540 \\
\hline d sh shale & $\begin{array}{r}23 \\
4\end{array}$ & $\begin{array}{l}1,352 \\
1,356\end{array}$ & $\begin{array}{l}\text { Brown sticky shale -- } \\
\text { Sondy shale and }\end{array}$ & 5 & 2,545 \\
\hline ticky black shale .... & 14 & 1,370 & boulders & 75 & 2,620 \\
\hline ard sand & 5 & 1,375 & Sand & 5 & 2,625 \\
\hline $\begin{array}{l}\text { nale and streaks (of) } \\
\text { sand }\end{array}$ & 8 & 1.383 & $\begin{array}{l}\text { Sticky shale } \\
\text { Hard sand and gravel. }\end{array}$ & $\begin{array}{r}30 \\
2\end{array}$ & $\begin{array}{l}2,655 \\
2,657\end{array}$ \\
\hline & 67 & 1,450 & Sticky blue shale -.. & 13 & 2,670 \\
\hline ticky shale, blue -... & 40 & 1,490 & Sticky shale and & & \\
\hline t1cky shale & 10 & 1,500 & sand and gravel ...- & 30 & 2,700 \\
\hline & 10 & 1,510 & Sandy shale and shale & 15 & 2,715 \\
\hline lcky shale & 10 & 1,520 & Blue shale & 5 & 2,720 \\
\hline $\begin{array}{l}\text { 1cky blue shale } \\
\text { hale and sand }\end{array}$ & 35 & 1,555 & Sandy shale & $\stackrel{31}{C_{0}}$ & 2,751 \\
\hline & & & & & \\
\hline
\end{tabular}


30/38-19P2.--Cont inued

\begin{tabular}{|c|c|c|c|c|c|}
\hline Material & $\begin{array}{l}\text { kness } \\
\text { eet) }\end{array}$ & $\begin{array}{l}\text { Depth } \\
\text { (feet) }\end{array}$ & $\begin{array}{r}\text { Thickn } \\
\text { (fee }\end{array}$ & $\begin{array}{l}\text { ness } \\
\text { et) }\end{array}$ & $\begin{array}{l}\text { Depth } \\
\text { (feet) }\end{array}$ \\
\hline $\begin{array}{l}\text { Sandy shale, streaks } \\
\text { hard sand }\end{array}$ & 6 & 2,757 & Tough sticky shale... & $\begin{array}{r}62 \\
2\end{array}$ & $\begin{array}{l}3,640 \\
3.642\end{array}$ \\
\hline Blue shale and lime & 4 & 2,761 & Tough sticky shale .. & 31 & \\
\hline Shale $\ldots \ldots$ & 9 & 2,770 & Hard sand ................ & 2 & 3,675 \\
\hline ard & 1 & 2,771 & Shale and streaks & & \\
\hline icky shale ..... & 49 & 2,820 & of sand & 25 & 3,700 \\
\hline and $\cdots$ & 23 & 2,843 & No entry & 9 & 3,709 \\
\hline Sand and boulders & 20 & 2,863 & Sticky shale & 15 & 3,724 \\
\hline Blue sandy shale. & 5 & 2,868 & Sand $\ldots$ & 3 & 3,727 \\
\hline Sand & 30 & $2,898 i$ & Sand and shale & 9 & 3,736 \\
\hline ticky blue shale & 3 & 2,901 & Sticky blue shale & 10 & 3,746 \\
\hline ough sticky blue & 36 & 2,937 & 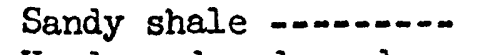 & 4 & 3,750 \\
\hline ticky blue shale & 16 & 2,953 & Hard sand and sandy & & \\
\hline Sand & 6 & 2,959 & shale & 15 & 3,765 \\
\hline $\begin{array}{l}\text { Sandy shale and } \\
\text { boulders }\end{array}$ & 9 & 2,968 & $\begin{array}{l}\text { Sandy shale with } \\
\text { streaks of streaky }\end{array}$ & & \\
\hline Sandy shale & 124 & 3,092 & shale & 15 & 3,780 \\
\hline $\begin{array}{l}\text { Sticky shale } \\
\text { Sandy shale, streaks }\end{array}$ & 15 & 3,107 & $\begin{array}{l}\text { Sandy brown shale } \\
\text { Sandy brown shale, }\end{array}$ & 17 & 3,797 \\
\hline hard sand & 35 & 3,142 & streak sand & 11 & 3,808 \\
\hline $\begin{array}{l}\text { Sticky shale - } \\
\text { Sandy shale wi }\end{array}$ & 14 & 3,1 & Brown & & \\
\hline $\begin{array}{l}\text { shale with } \\
\text { reaks hard sa }\end{array}$ & 34 & 3,190 & streaky shale & 8 & 3,816 \\
\hline Sandy shale & 10 & 3,200 & shells & 3 & 3,819 \\
\hline Sticky blue shale & 5 & 3,205 & Brown shale and streaks & & \\
\hline Hard sandy shale & 5 & 3,210 & of hard sand & 18 & 3,837 \\
\hline Sandy shale & 4 & 3,2 & Brown shale $\ldots . .$. & 10 & 347 \\
\hline Gard sand & 10 & & Sticky shale & 5 & 52 \\
\hline Sticky blue shale & 12 & 36 & Sandy shale, tough -. & 13 & 65 \\
\hline Sand & 1 & & Sandy shale & 10 & 875 \\
\hline Sandy shale -.- & 10 & & Sand & 4 & \\
\hline Tough sticky sh & 10 & 3,257 & Sandy shale & 7 & \\
\hline Sticky shale -- & 19 & & Sticky shale & 23 & 3,909 \\
\hline Coarse sand & 7 & & Tough sticky shale -- & 4 & 3,913 \\
\hline Hard sandy shale & 10 & & No entry & 4 & 3,917 \\
\hline Sticky shale & 20 & 3,313 & Blue shale & 18 & 35 \\
\hline Sendy shale, streaks & & & Hard sand & 1 & \\
\hline$f$ hard sand & 64 & 3,377 & Blue shale & 2 & \\
\hline Sandy shale & 10 & & Sticky shale & 8 & \\
\hline Blue sticky shale & 10 & & Blue shale ...... & 5 & \\
\hline Sendy shale & 6 & 3,403 & Sticky shale & 10 & \\
\hline Sandy shale and hard & & & Shale and lime & 25 & \\
\hline & $\begin{array}{l}23 \\
16\end{array}$ & & Sticky shale & 4 & \\
\hline $\begin{array}{l}\text { Sticky blue shale } \\
\text { Tough sticky shale }\end{array}$ & $\begin{array}{l}16 \\
65\end{array}$ & & Blue shale & 32 & \\
\hline $\begin{array}{l}\text { Tough sticky shale } \\
\text { Sandy shale }\end{array}$ & $\begin{array}{l}65 \\
12\end{array}$ & & Tough sticky shale .- & 3 & \\
\hline $\begin{array}{l}\text { Sandy shale } \\
\text { Tough sticky shale }\end{array}$ & 12 & & Sticky shale & 20 & \\
\hline $\begin{array}{l}\text { Tough sticky shale } \\
\text { Sandy shale }\end{array}$ & $\begin{array}{r}11 \\
8\end{array}$ & $\begin{array}{l}3,530 \\
3,538\end{array}$ & Brown sandy shale -.- & 15 & 4,060 \\
\hline Sticky blue shale ..... & 17 & 3, & sticky shale & 5 & 65 \\
\hline $\begin{array}{l}\text { Hard sand } \\
\text { Sticky shale }\end{array}$ & 2 & 3, & Hard sand & 5 & \\
\hline & & & & Cont & \\
\hline
\end{tabular}


30/38-19P2.--Cont Inued

\begin{tabular}{|c|c|c|c|c|c|}
\hline Material & $\begin{array}{l}\text { ness } \\
\text { et) }\end{array}$ & $\begin{array}{l}\text { Depth } \\
\text { (feet) }\end{array}$ & $\begin{array}{r}\text { Thickn } \\
\text { (fee }\end{array}$ & $\begin{array}{l}\text { hess } \\
\text { et) }\end{array}$ & $\begin{array}{l}\text { Depth } \\
\text { (feet) }\end{array}$ \\
\hline Hard brown sandy shale -- & 10 & 4,080 & Blúe shale and lime -. & 82 & 4,870 \\
\hline Hard brown shale & 14 & 4,094 & Dark shale and lime .. & 10 & 4,880 \\
\hline Sticky shale & 4 & 4,098 & Dark shale, some sand- & 8 & 4,888 \\
\hline Hard sandy shale & 6 & 4,104 & Blue shale, some sand- & 12 & 4,900 \\
\hline Brown shale and lime & 7 & 4,111 & Sticky shale, brown, & & \\
\hline Hard sand and line & 4 & 4,115 & some sand ............. & 7 & 4,907 \\
\hline Hard brown shale & 20 & 4,135 & Brown sandy shale ...- & 2 & 4,909 \\
\hline Hard brown shale and & & & Sticky brown shale ..- & 5 & 4,914 \\
\hline lime & 15 & 4,150 & Sticky brown shale and & & \\
\hline Sticky brown shale - & 9 & 4,159 & lime & 10 & 4,924 \\
\hline Stticky shale & 2 & 4,161 & Dark shale, some lime- & 4 & 4,928 \\
\hline Sticky blue shale & 7 & 4,168 & Blue shale and IIme -- & 7 & 4,935 \\
\hline Sandy shale & 18 & 4,186 & Dark shale, little & & \\
\hline Sticky shale & 11 & 4,197 & Ilme & 22 & 4,957 \\
\hline Sticky blue shale ...... & 3 & 4,200 & Shale and lime & 6 & 4,963 \\
\hline Sticky shale & 6 & 4,206 & Sticky shale and lime- & 10 & 4,973 \\
\hline Brown shale & 5 & 4,211 & Brown shale and lime-- & 13 & 4,986 \\
\hline $\begin{array}{l}\text { Sticky shale } \\
\text { Sticky blue shale and }\end{array}$ & 13 & 4,224 & St 1cky brown shale, & & \\
\hline $\begin{array}{l}\text { Sticky blue shale and } \\
\text { lime }\end{array}$ & 5 & 4,229 & $\begin{array}{l}\text { some lime } \\
\text { Derk shale }\end{array}$ & $\begin{array}{l}0 \\
9\end{array}$ & $\begin{array}{l}4,994 \\
5,003\end{array}$ \\
\hline Tough blue shale and & & & Blue shale, slightly & & \\
\hline IIme & 18 & 4,247 & sandy $\ldots \ldots \ldots$ & 7 & 5,010 \\
\hline St1cky shale and lime -- & 8 & 4,255 & Hard dry sand ............. & 5 & 5,015 \\
\hline Blue shale and lime ..... & 75 & 4,330 & Brown shale $\ldots \ldots \ldots . .$. & 3 & 5,018 \\
\hline Brown shale & 12 & 4,342 & Brown shale, some lime & 5 & 5,023 \\
\hline Blue shale and lime ... & 42 & 4,384 & Brown shale and lime-- & 7 & 5,030 \\
\hline Lime rock & 1 & 4,385 & Brown shale, streaks & & \\
\hline Rock and IIme shell & 6 & 4,391 & of sand & 8 & 5,038 \\
\hline Blue shale and lime & 51 & 4,442 & Brown shale, some & & \\
\hline Sticky shale, some lime-- & 5 & 4,447 & sand and lime & 6 & 5,044 \\
\hline $\begin{array}{l}\text { Brown sandy shale and } \\
\text { lime }\end{array}$ & 18 & 4,465 & Brown shale and lime-- & & \\
\hline Brown shale and lime .... & 9 & 4,474 & & & \\
\hline Shale and line & 9 & 4,483 & & & \\
\hline Blue shale and lime ..... & 189 & 4,672 & & & \\
\hline $\begin{array}{l}\text { Brown shale and Iime } \\
\text { St1cky blue shale and }\end{array}$ & 28 & 4,700 & & & \\
\hline $\begin{array}{l}\text { St1cky blue shale and } \\
\text { lime }\end{array}$ & 7 & 4,707 & & & \\
\hline Blue shale and lime & 20 & 4,727 & & & \\
\hline $\begin{array}{l}\text { Sticky brown shale, some } \\
\text { lime }\end{array}$ & & & & & \\
\hline Brown shale and lime ..... & 60 & 4,788 & & & \\
\hline
\end{tabular}


30/38-30PI. M and $R$, Cant Il Ranch, well 1. fltitude about 1,960 feet. 20-inch casing zero to 150 feet, 12-inch casing 150 to 643 feet; perforated 130 to 150 and 153 to 643 feet.

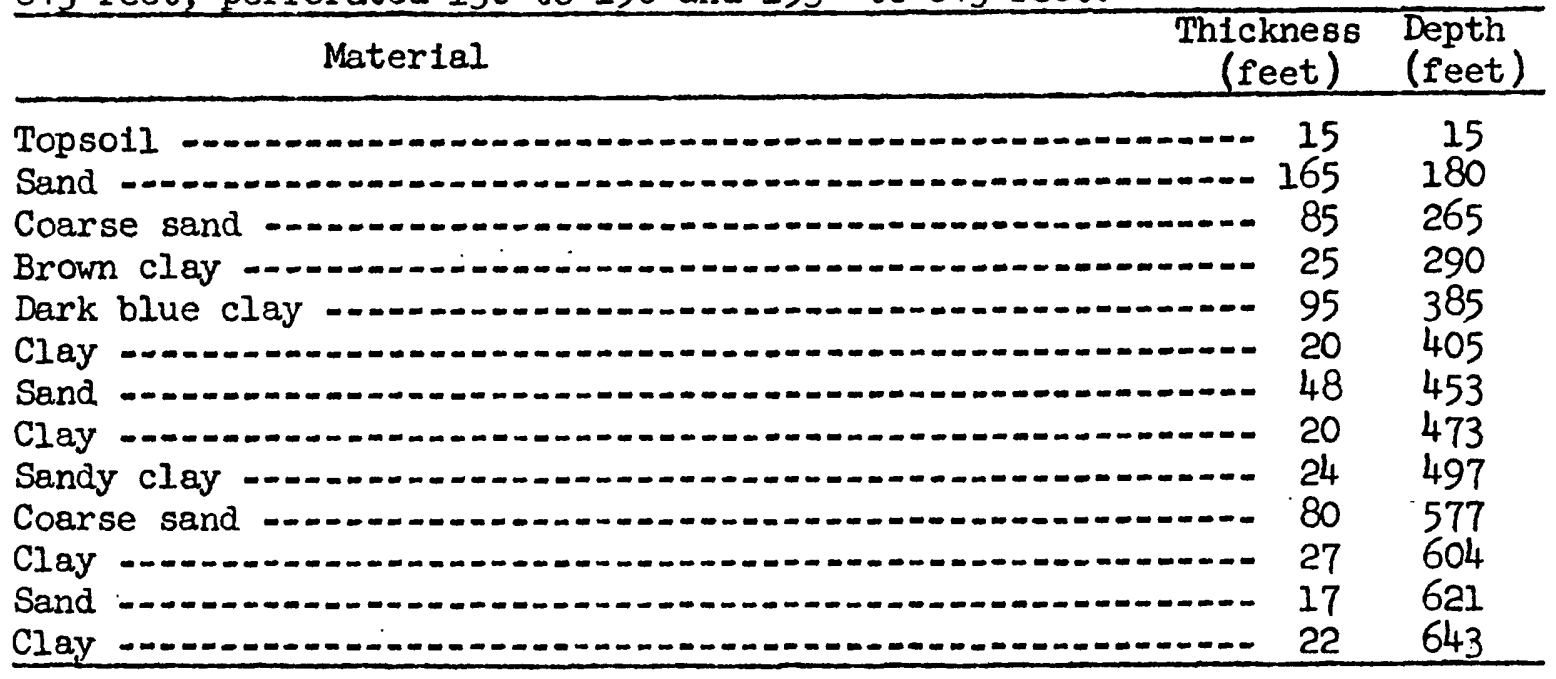

30/38-3lFI. M and R, Cantil Ranch, well 3. Altitude about 1,990 feet. 16-inch casing zero to 196 feet, 12-inch casing 196 to 658 feet; perforated 118 to 196 and 202 to 658 feet.

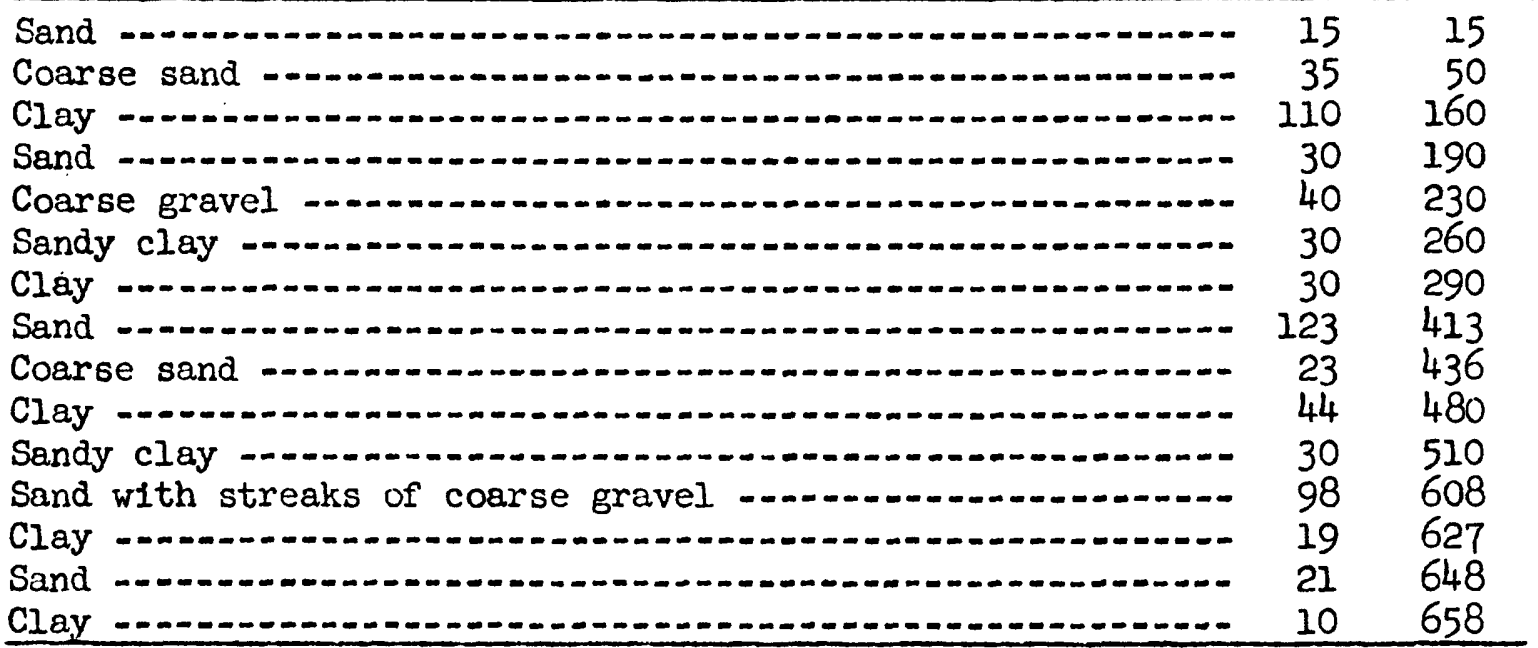

30/38-3lGl. M and R, Cant1l Ranch, well 2. Altitude about 1,990 feet. 20-inch casing zero to 156 feet, 12 -inch casing 156 to 656 feet; perforated 120 to 656 feet.

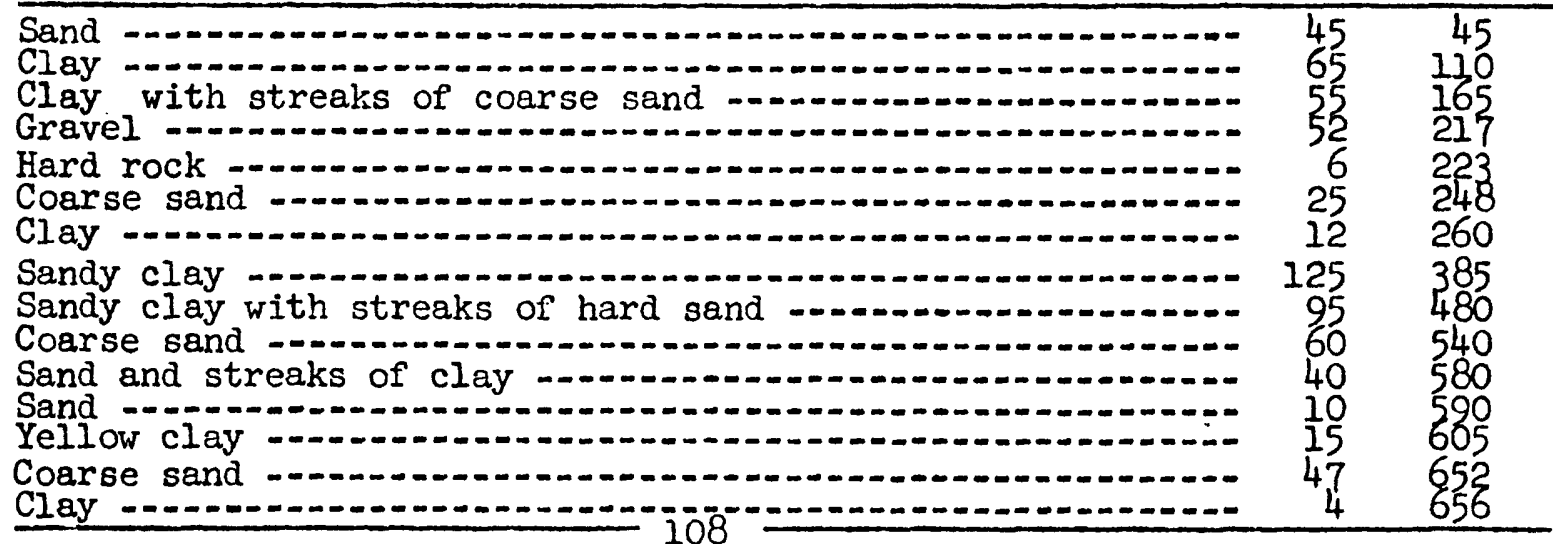


30/38-32Gl. M and $R$, Cantil Ranch, well 8. Altitude 1,949.0 feet. 20-inch casing zero to 408 feet, 12-inch casing 408 to 852 feet; perforated 120 to 408 and 414 to 852 feet; uncased hole 852 to 863 feet.

\begin{tabular}{|c|c|c|}
\hline Material & $\begin{array}{c}\text { hickness } \\
\text { (feet) }\end{array}$ & $\begin{array}{l}\text { Depth } \\
\text { (feet) }\end{array}$ \\
\hline $\begin{array}{l}\text { Topsoll and clay } \\
\text { Red sand with streaks of clay } \\
\text { Blue clay } \\
\text { Blue-gray sand with streaks of clay } \\
\text { Yellow clay } \\
\text { Red sand } \\
\text { Yellow clay } \\
\text { Yellow sandy clay } \\
\text { Coarse sand } \\
\text { Hard sand }\end{array}$ & 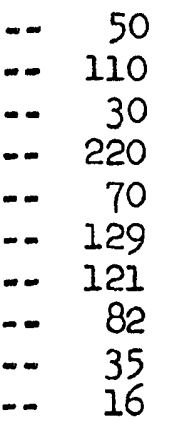 & $\begin{array}{l}50 \\
160 \\
190 \\
410 \\
480 \\
609 \\
730 \\
812 \\
847 \\
863\end{array}$ \\
\hline
\end{tabular}

30/39-3Cl. James Stocton. Alt1tude about 2,140 feet. Dr1lled by D. W. Slocum in July 1956. 14-1nch casing zero to 600 feet, perforated 234 to 600 feet; uncased hole 600 to 610 feet.

\begin{tabular}{|c|c|c|c|c|}
\hline $\begin{array}{c}\text { Thickness } \\
\text { (feet) }\end{array}$ & $\begin{array}{l}\text { Depth } \\
\text { (feet) }\end{array}$ & Materia & $\begin{array}{c}\text { Thickness } \\
\text { (feet) }\end{array}$ & $\begin{array}{l}\text { Depth } \\
\text { (feet) }\end{array}$ \\
\hline Sandy soil $\ldots \ldots \ldots$ & 3 & Yellow & sandy clay .... 4 & 362 \\
\hline Sand rock and gravel .... 17 & 20 & Coarse & sand $\ldots . . . . . . . .$. & 368 \\
\hline Coarse sand ........... 15 & 35 & Yellow & sandy clay .... & 378 \\
\hline Yellow sandy clay ....... 5 & 40 & Coarse & sand $\ldots . . . . . . . .$. & 385 \\
\hline Coarse sand and rock .... 20 & 60 & Yellow & sandy clay .... & 402 \\
\hline Yellow sandy clay $\ldots \ldots \ldots$ & 64 & Coarse & sand ............... & 410 \\
\hline Coarse sand and gravel-.. 17 & 81 & Yellow & clay & 426 \\
\hline Yellow sandy clay ...... Il & 92 & Coarse & sand & 433 \\
\hline Coarse sand and rocks ... 22 & 124 & Yellow & clay ............... & $4 \overline{66}$ \\
\hline Sandy clay and rocks .... 22 & 136 & Coarse & sand & 452 \\
\hline Coarse sand and gravel-.. 5 & 141 & Yellow & clay $\ldots \ldots \ldots \ldots$ & 460 \\
\hline Sandy clay and rocks .... 24 & 165 & Coarse & sand & 468 \\
\hline Coarse sand and gravel-.. 7 & 172 & Yellow & clay & 475 \\
\hline Sandy clay and rocks .... 18 & 190 & Coarse & sand and gravel & 484 \\
\hline Coarse sand and rocks ... 8 & 198 & Yellow & clay (sandy) -. & 498 \\
\hline Sandy clay and rocks .... 12 & 210 & Coarse & sand ................. & 505 \\
\hline Coarse sand and gravel-.. 6 & 216 & Yellow & sandy clay .... & 520 \\
\hline Sandy clay and rocks _... 14 & 230 & Coarse & sand - . . . & 528 \\
\hline Coarse sand and gravel... 8 & 238 & Yellow & clay -.......... & 540 \\
\hline Sandy clay and gravel _.. 12 & 250 & Coarse & sand & 546 \\
\hline Coarse sand $\ldots$ & 255 & Yellow & clay .............. & 558 \\
\hline Yellow clay ............ 10 & 265 & Coarse & sand & 564 \\
\hline Coarse sand & 270 & Yellow & 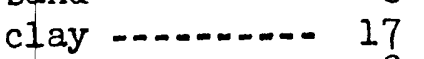 & 581 \\
\hline Yellow clay & 282 & Coarse & sand $\ldots \ldots . . . . . . .$. & 589 \\
\hline Coarse sand & 288 & Yellow & sandy clay .... & 594 \\
\hline Yellow clay ........... 13 & 301 & Coarse & sand ....... & 598 \\
\hline Coarse sand ....... & 307 & Yellow & sandy clay. & 604 \\
\hline Yellow clay & 312 & Coarse & sand $\ldots \ldots$ & 610 \\
\hline Coarse sand and rocks ... 6 & 318 & & & \\
\hline Yellow clay ........... 12 & 330 & & & \\
\hline Coarse sand and rock .... 5 & 335 & & & \\
\hline Yellow clay ............ 15 & 350 & & & \\
\hline Coarse sand .......... & 358 & & & \\
\hline
\end{tabular}


31/37-1Hn. $M$ and $R$, Cantil Ranch, well 16. Alt1tude about 2,030 feet. 14-1nch casing zero to 504 feet, perforated 252 to 504 feet.

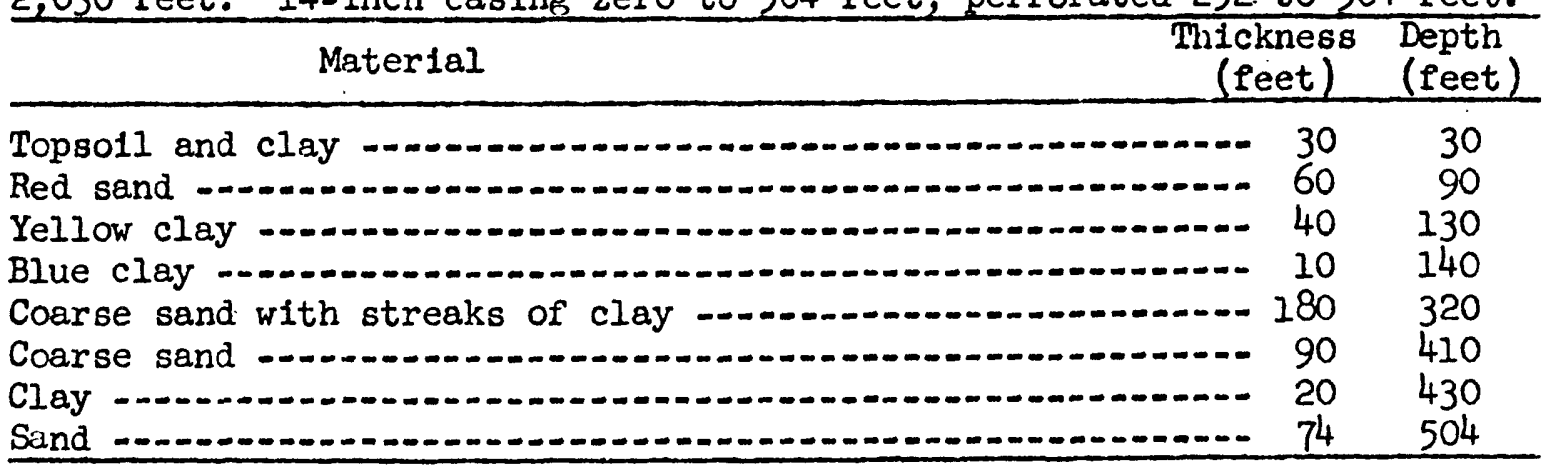

31/37-1Rl. $M$ and $R$, Cantil Ranch, well Il. Alt1tude about 2,065 feet. 20-inch casing zero to 402 feet, 12-Inch casing 402 to 468 feet; perforated 240 to 402 and 408 to 468 feet.

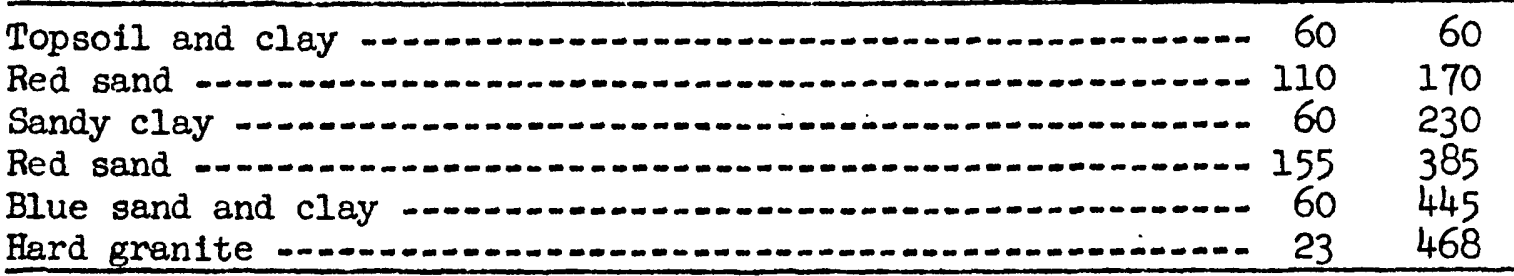

31/37-2PI. $M$ and $R$, Cant1l Ranch well 6. Alt1tude about 2,080 feet. 18-inch casing zero to 324 feet, perforated 120 to 324 feet; reducer 324 to 330 feet; 12-inch perforated casing 330 to 380 feet.

Topsoil and sand -...................................... 1010

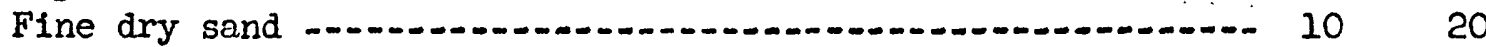

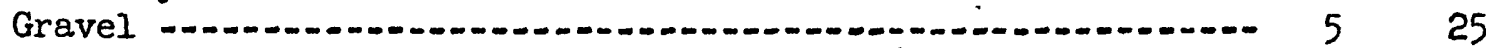

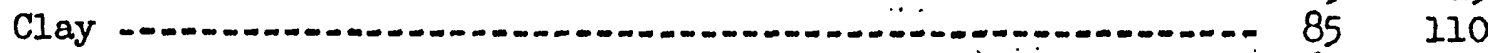

Clay with streaks of sand and gravel ...................... 69179

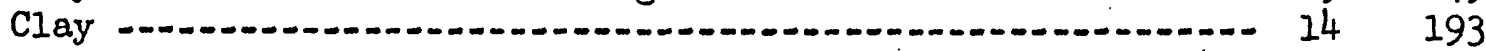

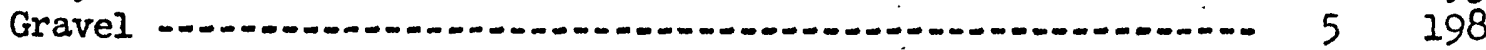

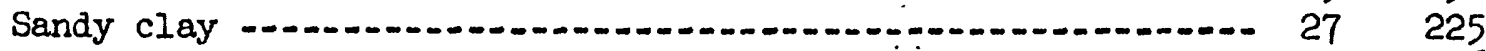

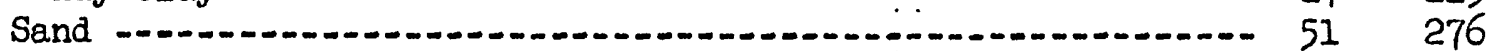

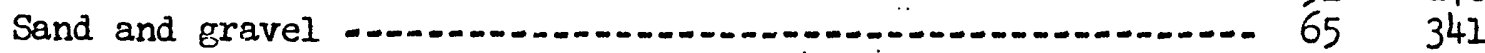

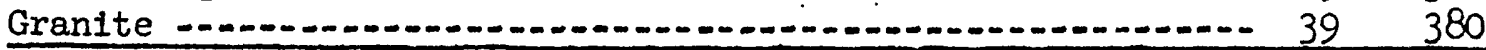

31/37-22J. Cinco, well I, oil test. Altitude about 2,235 feet. 10-inch surface casing.

Coarse sand ............................................ 4040

Sand and sandy shale -......................................... 98

Sand and sticky shale ........................................ $34 \quad 172$

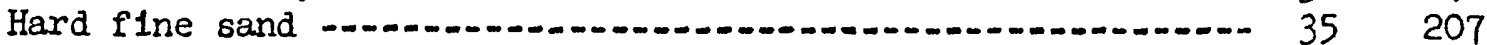

Sandy shale and bentonite ................................... 50212

Gray sand carrying water -................................... $58 \quad 270$

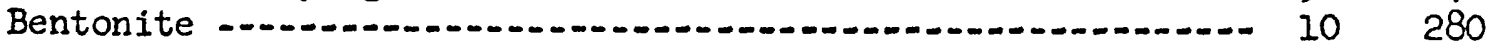

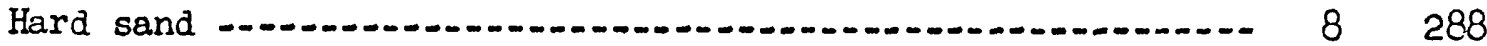

Hard sand, streaks of bentonite ............................ 49337

Continued 
31/37-22J1..-Continued

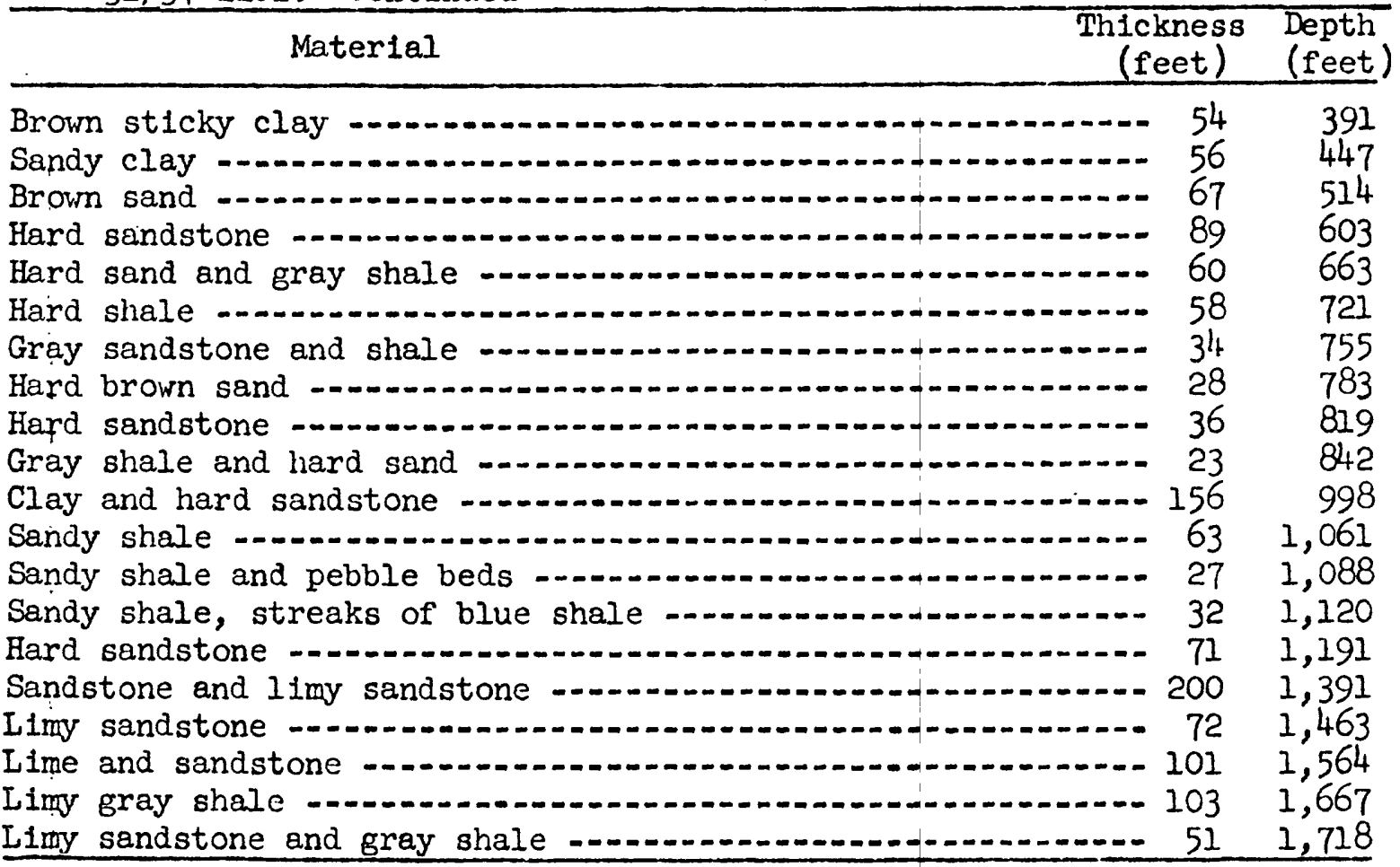

31/37-22RI. H1x, well 1, oll test. Altitude about 2,240 feet. Drilled by Cinco Development Co, 12-inch surface casing.

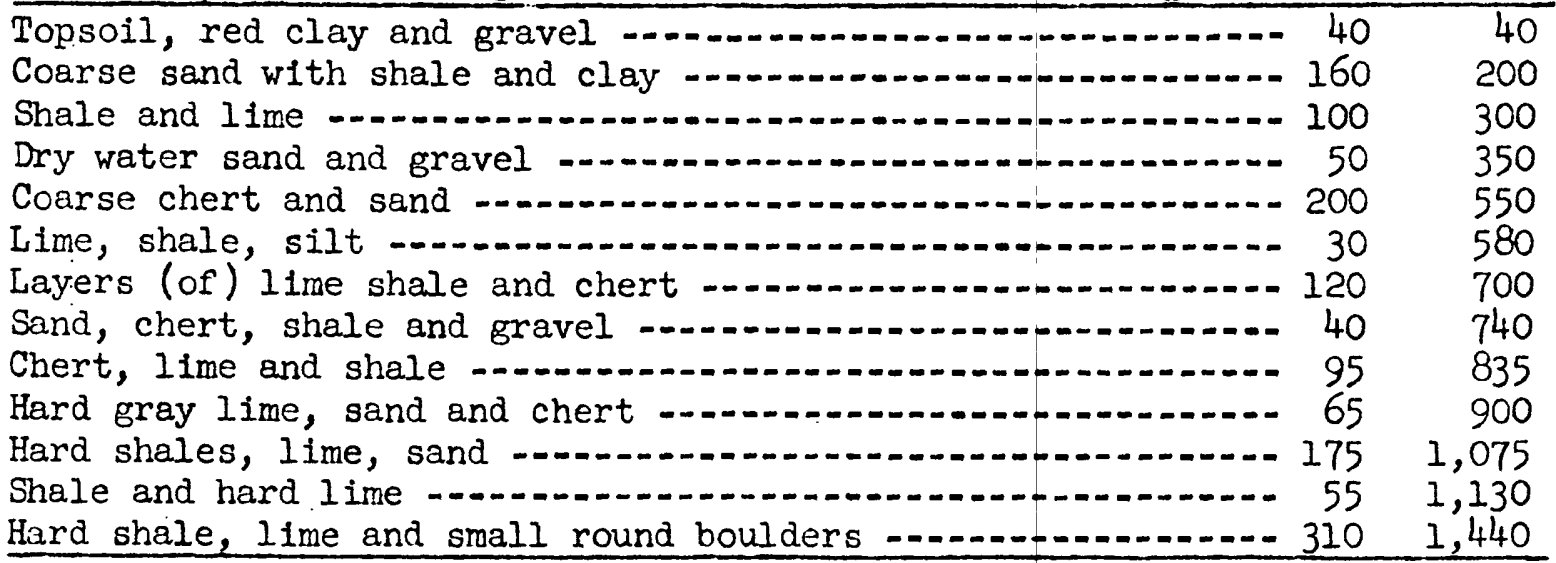


31/38-22H. Fremont 011 Syndicate, well 1, oil test. Alt1tude about 2,660 feét. Drilled by Fremont 0il Corp. 8-1nch surface casing.

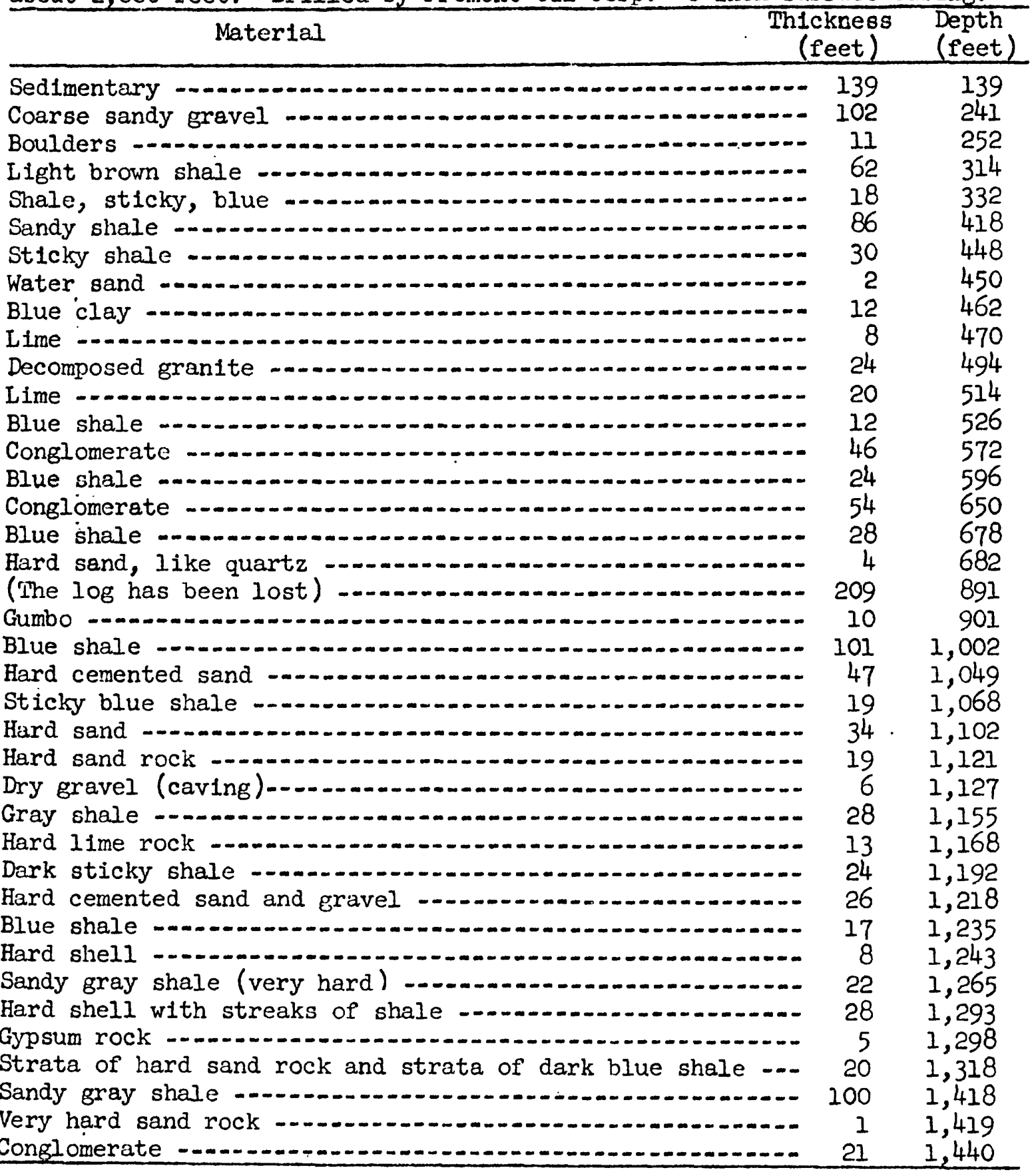


31/38-22H2. Fremont 0il Syndicate, well 2, oil test. Altitude about 2,655 feet. Drilled by Fremont 011 Corp. 5-inch surface casing.

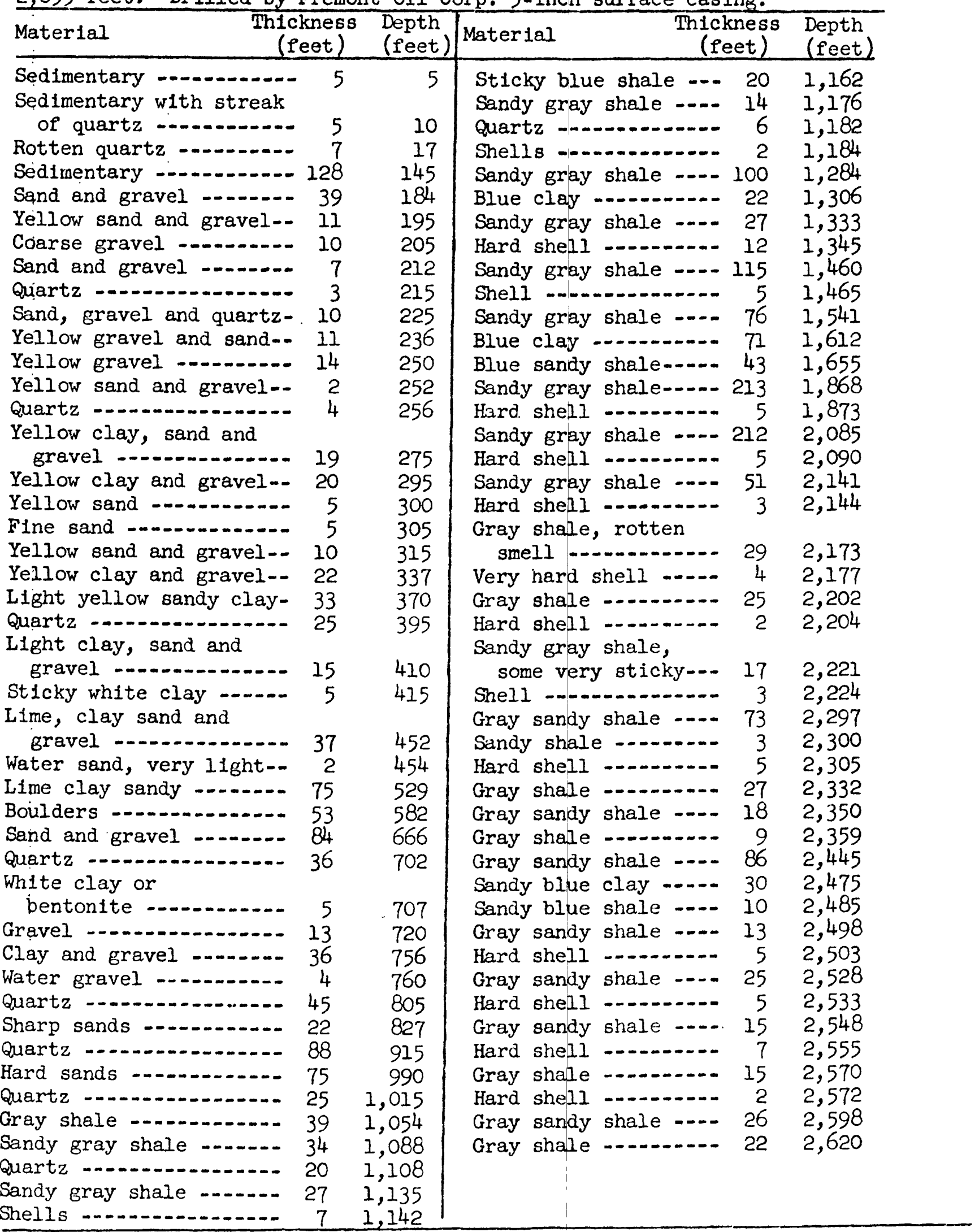


32/36-22BI. Oliver Pesch. Alt1tude about 2,710 feet. Drilled by W. H. Colquitt in October 1947. 6-inch casing zero to 713 feet, uncased hole 713 to 829 feet.

\begin{tabular}{|c|c|c|}
\hline Material & $\begin{array}{c}\text { Thickness } \\
\text { (feet) }\end{array}$ & $\begin{array}{l}\text { Depth } \\
\text { (feet) }\end{array}$ \\
\hline $\begin{array}{l}\text { Not logged } \\
\text { Yellow clay streaks and loose gravel streaks } \\
\text { Clean coarse sand } \\
\text { Not logged }\end{array}$ & $\begin{array}{l}-- \\
- \\
-\end{array}$ & $\begin{array}{l}535 \\
605 \\
700 \\
829\end{array}$ \\
\hline
\end{tabular}

32/37-9-1. $M$ and $R$, Conklin Ranch, formerly J. S. and $L$. Exploration Co., Childs Wall, well l, oll test. Alt1tude about 2,445 feet. Casing record not available.

\begin{tabular}{|c|c|c|}
\hline Alluvial sand and clay $\ldots \ldots \ldots$ & 202 & 202 \\
\hline Clay & 3 & 205 \\
\hline Sand & 5 & 210 \\
\hline Sandy clay and boulders & 90 & 300 \\
\hline Clay & 20 & 320 \\
\hline 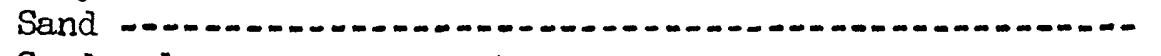 & 10 & 330 \\
\hline Sandy clay & 15 & 345 \\
\hline Sand and streak of & 65 & 410 \\
\hline Hard shell & 10 & 420 \\
\hline Water sand & 30 & 450 \\
\hline Hard clay & 40 & 490 \\
\hline Hard sand, streak of hard shale & 180 & 670 \\
\hline Herd shale & 40 & 710 \\
\hline Hard sand & 8 & 718 \\
\hline 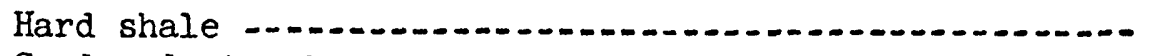 & 12 & 730 \\
\hline shale n- & 70 & 800 \\
\hline Hard shale & 60 & 860 \\
\hline Hard sand & 15 & 875 \\
\hline Hard sandy shale & 135 & 1,010 \\
\hline Hard cemented sand & 190 & 1,200 \\
\hline Sandy shale & 50 & 1,250 \\
\hline 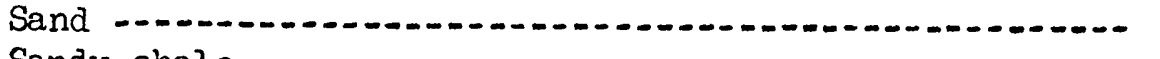 & 25 & 1,275 \\
\hline Sandy shale & 35 & 1,310 \\
\hline Sand and boulders & 70 & 1,380 \\
\hline Hard sand - . . . . & 20 & 1,400 \\
\hline Sandy shale & 75 & 1,475 \\
\hline 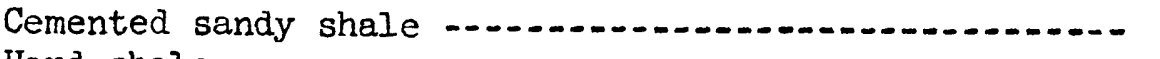 & 35 & 1,510 \\
\hline 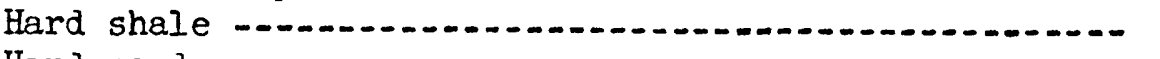 & 52 & 1,562 \\
\hline 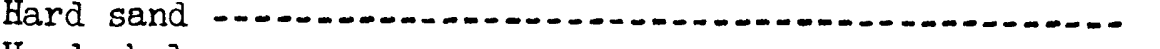 & 52 & 1,614 \\
\hline shale & 67 & 1,681 \\
\hline Hard sandy shale & 22 & 1,703 \\
\hline $\begin{array}{l}\text { Hard sand } \\
\text { Hard sandy streaked shale }\end{array}$ & 33 & 1,736 \\
\hline 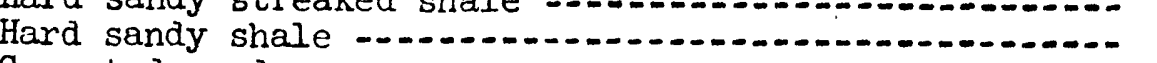 & 46 & $\begin{array}{l}1,805 \\
1,851\end{array}$ \\
\hline Cemented sand & 89 & 1,940 \\
\hline d sandy shale & 35 & 1,975 \\
\hline 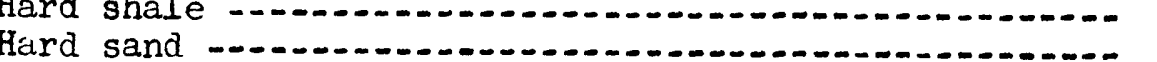 & 17 & 1,992 \\
\hline Id sandy shale & $\begin{array}{l}22 \\
20\end{array}$ & 2,014 \\
\hline Ird shale sand & 30 & 2,073 \\
\hline lard sandy shale & 85 & 2,107 \\
\hline $\begin{array}{l}\text { lard sand and boulders } \\
\text { lard shale }\end{array}$ & 17 & 2,204 \\
\hline
\end{tabular}


32/37-16Rl. $M$ and $R$, Conklin Ranch, well 14. Alt1tude about 2,440 feet. Drilled by Jess Goforth. 16-inch casing.

\begin{tabular}{|c|c|c|}
\hline Material & et) & $\begin{array}{l}\text { Depth } \\
\text { (feet) }\end{array}$ \\
\hline 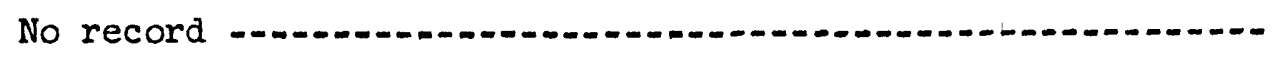 & -- & 205 \\
\hline Large - to medium-grained sand & 30 & 235 \\
\hline Intermittent streaks of sand and clay & 20 & 255 \\
\hline Streaks of gravel & 20 & 275 \\
\hline Large streaks of gravel & 40 & 315 \\
\hline Definitely a boulder conglomerate, basal in character & 5 & 320 \\
\hline Dense fine-grain varicolored sand & 20 & 340 \\
\hline Small streaks of clay and a fine unconsolldated & & \\
\hline varicolored sand & 40 & 380 \\
\hline $\begin{array}{l}\text { Large streaks of clay } \\
\text { Medium- to fine-grained sand and streaks, good water }\end{array}$ & 15 & 395 \\
\hline possibility & 35 & 430 \\
\hline Small streaks of gravel and small sand & 30 & 460 \\
\hline $\begin{array}{l}\text { Medium- to fine-gralned varlcolored sand with small } \\
\text { streaks of gravel, good water possibility }\end{array}$ & 45 & 505 \\
\hline Small streaks of gravel and small rocks & 40 & 545 \\
\hline $\begin{array}{l}\text { A medium- to very fine-grained sand, crystals predominate, } \\
\text { some quartz crystals, some rounded small sand, excellent }\end{array}$ & & \\
\hline $\begin{array}{l}\text { water possibllity } \\
\text { Clay content more than } 50 \text { percent in well-consolidated }\end{array}$ & 40 & 585 \\
\hline sand & 30 & 615 \\
\hline Some streaks of unconsolidated sand ...... & 30 & 645 \\
\hline Streaks of clay and a medium sand .......... & 41 & 686 \\
\hline
\end{tabular}

32/37-26M. $M$ and $M$, Conklin Ranch, well 15. Altitude about 2, 420 feet. Drilled by Jess Goforth in 1953. 16-inch casing.

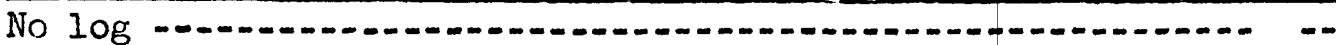

A small streak of coarse gravel ....................... 10

A medium- to small-grain send, varicolored and more

rounded than above, water possibllity _............ $25 \quad 265$

Alternate streaks of clay and sand ..................... $25 \quad 290$

Gravel and small boulders with streaks of clay .......... $25 \quad 315$

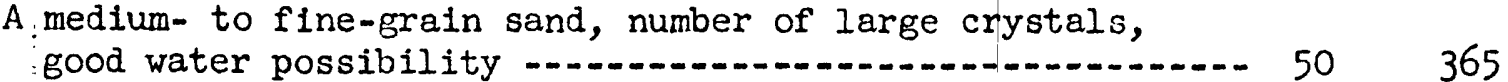

Medium- to fine-grain sand, clay streaks ............. $25 \quad 390$

As above, with more clay streaks ...... 7397

Mostly clay streaked with small sand

A few streaks of small boulders ............................ 10

Medium- to fine-grain sand, alternating with small
gravel

A medium- to fine-grain sand, varicolored, small grains

are well rounded and mainly clear and black minerals,

excellent water possibility _............. $75 \quad 535$

A very hard streak of well-cemented sand and gravel ....... 15

A varicolored large to medium sand, some crystals well

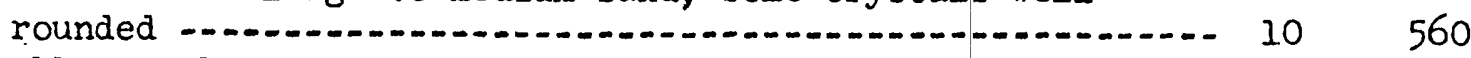

Small gravel and large-gralned sand, good water posslbility; cuttings from bottom are large sand, sharp, many flakes from dark and clear minerals _....................... $38 \quad 598$ 
32/39-4LI. J. E. Johnson Co., $M$ and $R$ well 1 , oil test. Altitude about 2,725 feet. 10-Inch gurface casing.

Material

Thickness (feet)

Alluvial sand and hard shells

Hard sand and shells

Sand and hard streaks

Sandy clay, streaks of gravel

Sandy shale -...

Hard blue shale

Sticky shale

Sandy shale

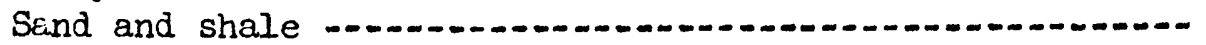

Sandy shale and streaks of gravel

Tight sand and boulders

Hard sand

Cemented decomposed granitic material

Decomposed granite and shale

Hard sand

Decomposed cemented granite

Tight coarse sand

Decomposed lime and granite

Hard sand

Decomposed lime and granite .......

Hard sand

Sandy shale -....

Hard sand -

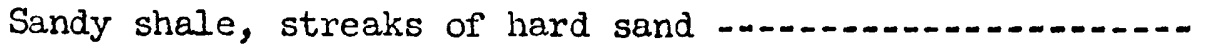

Decomposed lime and granite

\begin{tabular}{rr}
43 & 43 \\
218 & 261 \\
77 & 338 \\
250 & 588 \\
178 & 766 \\
67 & 833 \\
55 & 888 \\
132 & 1,020 \\
101 & 1,121 \\
63 & 1,184 \\
190 & 1,374 \\
23 & 1,397 \\
1 & 1,398 \\
107 & 1,505 \\
79 & 1,584 \\
32 & 1,616 \\
10 & 1,626 \\
82 & 1,708 \\
286 & 1,994 \\
123 & 2,117 \\
158 & 2,275 \\
48 & 2,323 \\
33 & 2,356 \\
26 & 2,382 \\
10 & 2,392 \\
\hline
\end{tabular}

32/40-31Bl. U. S. Borax and Chemical Corp., well 22. Altitude about 2,530 feet. Drilled by owner in April 1954. Uncased hole zero to 290 feet.

Sand, clay and gravel, hard, moderately well cemented ... 160160 Sand and gravel with thin zones of brown clay ........ $15 \quad 175$ Sand, clay and gravel; well mixed, brown, hard ........ $60 \quad 235$ Clay, brown, hard, sandy, streaks of coarse water gravel at 260 feet ........... $30 \quad 265$

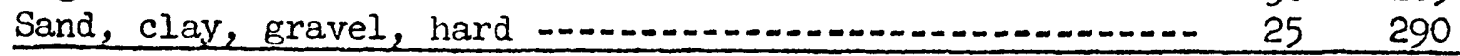


32/40-3IFI. U. S. Borax and Chemical Corp., well 23. Altitude about 2,520 feet. Drilled by owner in April 1954. Uncased hole zero to 500 feet. Material Thickness Depth (feet) (feet)

Sand, clay and gravel; sand granitic, clay browh, well mixed, compact, hard ...................................... 170170

Sand and gravel; water bearing ............................... 5

Sand, clay and gravel; brown, well mixed, hard, compact ...... $95 \quad 270$

Sand and gravel; some streaks of brown clay, gravel increasing dow section

Sand, clay and gravel, brown, well mixed ..................... 138

Sand; very coarse, poorly sorted, few pebbles and boulders ... $10 \quad 440$

Clay; sandy, gray ............................................ 344

Gravel; coarse, waterworn, some basalt pebbles, thin streaks of clay and sand -................................... 4447 Clay; sandy, gray -................................................ 13460

Sand and gravel, very coarse, poorly sorted, basalt and grantic boulders ........................................... 16476 Clay, blue-gray, sandy with occasional gravel streaks ....... $24 \quad 500$ 
Table 5.- Chemical analyses of water from wells

Constituents: There the value for sodium is preceded by the letter a it indicates sodium and potassium expressed as sodium. The value for dissolved solids is the analytically determined value reported by the laboratory. The sum of determined constituents is the sum of the tabulated constituents minus approximately half ( 50.8 percent) of the bicarbonate. Because all the major constituents (except silica in many of the analyses) that commonly occur in ground water were analytically determined, the values for dissolved solids and sum of determined constituents should be approximately the same. Constituents shown in parentheses are values calculated by the Geological Survey, Ground Water Branch. All values have been rounded where necessary to conform to the standards of the Geological Survey, Quality of Water Branch.

Temperature: For the Geological Survey analyses (GW, GP, and $Q W$ ), where the temperature is given the sample was collected from the pump discharge; where the temperature is omitted the samples were collected mainly from a storage facility at the well. For the other analyses the point of collection was mainly from the pump discharge.

Analyzing laboratory: DWR, State of California, Department of Water Resources; GP, U. S. Geological Survey, Geochemistry and Petrology Branch; GW, U. S. Geological Survey, Ground Water Branch; $\mathrm{H}$, HornkohI Co.; QW, U. S. Geological Survey, Quality of Water-Branch; SE, Smith-Emery Co. For analyses for which the analyzing laboratory is not given the agency from which the analysis was collected is given: CW, Cyril Williams, Jx. (1930); DGT, Thompson (1929); SP, Southern Pacific Co. 


Well number $\quad:_{10 / 7-5 D 1}: 11 / 7-30 G 1: 11 / 7-32 E 1: 11 / 7-32 K 1$

Constituents in parts per million

\begin{tabular}{|c|c|c|c|c|}
\hline $\begin{array}{l}\text { Silica }\left(\mathrm{SiO}_{2}\right) \\
\text { Iron }(\mathrm{Fe})\end{array}$ & 44 & 15 & $\begin{array}{c}51 \\
\text { Trace }\end{array}$ & 0.10 \\
\hline $\begin{array}{l}\text { Calcium (Ca) } \\
\text { Magnesium (Mg) } \\
\text { Sodium (Na) } \\
\text { Potassium (K) }\end{array}$ & $\begin{array}{l}48 \\
7.0 \\
330 \\
6.0\end{array}$ & $\begin{array}{r}49 \\
11 \\
215 \\
6.0\end{array}$ & $\begin{array}{r}72 \\
19 \\
356\end{array}$ & $\begin{array}{c}28 \\
4.9 \\
366\end{array}$ \\
\hline $\begin{array}{l}\text { Bicarbonate }\left(\mathrm{HCO}_{3}\right) \\
\text { Carbonate }\left(\mathrm{CO}_{3}\right)^{3} \\
\text { Sulfate }\left(\mathrm{SO}_{4}\right)^{3} \\
\text { Chloride }\left(\mathrm{Cl}^{4}\right)\end{array}$ & $\begin{array}{r}162 \\
17 \\
271 \\
302\end{array}$ & $\begin{array}{l}192 \\
223 \\
180\end{array}$ & $\begin{array}{r}220 \\
0 \\
345 \\
350\end{array}$ & $\begin{array}{r}168 \\
18 \\
310 \\
278\end{array}$ \\
\hline $\begin{array}{l}\text { Fluoride }(\mathrm{F}) \\
\text { Nitrate }\left(\mathrm{NO}_{3}\right) \\
\text { Boron }(\mathrm{B})\end{array}$ & $\begin{array}{r}.8 \\
5.0 \\
2.2\end{array}$ & $\begin{array}{l}1.6 \\
5.0 \\
1.3\end{array}$ & & 12 \\
\hline $\begin{array}{l}\text { Dissolved solids (Dis. S) } \\
\text { Sum of determined constituents } \\
\text { Hardness as } \mathrm{CaCO}_{3}\end{array}$ & $\begin{array}{r}(1,110) \\
(149)\end{array}$ & $\begin{array}{l}840 \\
(802) \\
(168)\end{array}$ & $\begin{array}{r}(1,300) \\
(258)\end{array}$ & $\begin{array}{c}1,090 \\
(1,100) \\
90\end{array}$ \\
\hline $\begin{array}{l}\text { Percent sodium (\%Na) } \\
\text { Specific conductance } \\
\left.\text { (micromhos at } 77^{\circ} \mathrm{F}\right) \\
\text { pH } \\
\text { Temperature }\left({ }^{\circ} \mathrm{F}\right)\end{array}$ & $\begin{array}{r}82 \\
1,810 \\
8.4\end{array}$ & $\begin{array}{r}74 \\
1,190 \\
7.8\end{array}$ & (75) & $\begin{array}{r}(90) \\
1,660 \\
8.3\end{array}$ \\
\hline $\begin{array}{l}\text { Date collected (Date) } \\
\text { Depth of well in feet (Depth) } \\
\text { Analyzing laboratory and number } \\
\text { (Lab., No.) }\end{array}$ & & $\begin{array}{l}4-22-53 \\
675 \\
\text { GP }\end{array}$ & $\begin{array}{l}6-11-56 \\
502 \\
\mathrm{SE}\end{array}$ & $\begin{array}{c}8.9-57 \\
684 \\
\mathrm{H}\end{array}$ \\
\hline
\end{tabular}




\begin{tabular}{|c|c|c|c|c|c|c|}
\hline $11 / 7-32 \mathrm{M} 2$ & $\begin{array}{l}\vdots \\
\vdots\end{array}$ & $-32 N 1$ & $11 / 7-32 N$ & $2: 11 / 7-32 N$ & $13: 11 / 7-3214$ & $11 / 8-2 M$ \\
\hline 0.10 & & 24.5 & 58 & 36 & & ${ }^{72} .06$ \\
\hline $\begin{array}{r}63 \\
11 \\
356\end{array}$ & & $\begin{array}{c}39 \\
7.7 \\
304 \\
0\end{array}$ & $\begin{array}{c}54 \\
8.0 \\
280 \\
9.0\end{array}$ & $\begin{array}{c}60 \\
9.5 \\
343\end{array}$ & $\begin{array}{r}46 \\
11 \\
295 \\
7.0\end{array}$ & $\begin{array}{c}30 \\
8.5 \\
125 \\
6.4\end{array}$ \\
\hline $\begin{array}{r}207 \\
0 \\
346 \\
316\end{array}$ & 195 & $\begin{array}{r}267 \\
0 \\
268 \\
191\end{array}$ & $\begin{array}{r}235 \\
0 \\
256 \\
231\end{array}$ & $\begin{array}{r}201 \\
0 \\
290 \\
323\end{array}$ & $\begin{array}{r}239 \\
0 \\
262 \\
218\end{array}$ & $\begin{array}{l}201 \\
87 \\
83\end{array}$ \\
\hline 20 & & $\underbrace{.8}_{1.5}$ & $\begin{array}{l}1.2 \\
20 \\
2.7\end{array}$ & $\frac{13^{.6}}{2.3}$ & $25^{.5}$ & $24^{.9} .60$ \\
\hline $\begin{array}{c}1,220 \\
(1,210) \\
202\end{array}$ & 145 & $\begin{array}{c}1,000 \\
(995) \\
129\end{array}$ & $\begin{array}{r}1,060 \\
(1,040) \\
(168)\end{array}$ & $\begin{array}{c}1,240 \\
(1,180) \\
188\end{array}$ & $\begin{array}{l}1,060 \\
(984) \\
(160)\end{array}$ & $\begin{array}{l}536 \\
(536) \\
110\end{array}$ \\
\hline $\begin{array}{r}(79) \\
1,560\end{array}$ & 1,590 & $\begin{array}{r}83 \\
1,590\end{array}$ & $\begin{array}{r}79 \\
1,530\end{array}$ & $\begin{array}{r}80 \\
1,910\end{array}$ & $\begin{array}{r}(79) \\
1,600\end{array}$ & $: \begin{array}{r}70 \\
805\end{array}$ \\
\hline $7: 4$ & & $\begin{array}{l}8.0 \\
70\end{array}$ & $\begin{array}{l}7 \cdot 7 \\
72\end{array}$ & 7.9 & 7.8 & $\begin{array}{l}8.2 \\
72\end{array}$ \\
\hline $\begin{array}{c}11-29-56 \\
530 \\
\text { H } 98527\end{array}$ & $\begin{array}{c}11-27-51 \\
305 \\
\mathrm{GW}\end{array}$ & $\begin{array}{l}4-2-52 \\
3055 \\
G P\end{array}$ & $\begin{array}{c}4-22-53 \\
454 \\
G P\end{array}$ & $\begin{array}{c}4-9-51 \\
400 \\
S E\end{array}$ & $\begin{array}{c}6-5-53 \\
375 \\
\text { DWR P-676 }\end{array}$ & $\begin{array}{c}4-2-52 \\
350 \\
\text { QW } 8529\end{array}$ \\
\hline
\end{tabular}




\begin{tabular}{|c|c|c|c|c|c|c|}
\hline & $: 11 / 8-2 \mathrm{~N} 1:$ & \multicolumn{2}{|c|}{$11 / 8-2 P 1$} & $: 11 / 8-2-1:$ & \multicolumn{2}{|c|}{$11 / 8-3 \mathrm{PI}: 11 / 8-10 \mathrm{RI}$} \\
\hline $\begin{array}{l}\mathrm{SiO}_{2} \\
\mathrm{Fe}\end{array}$ & & 60 & & & & \\
\hline $\mathrm{Ca}$ & 30 & 38 & 27 & & 34 & 37 \\
\hline $\mathrm{Mg}$ & 9.0 & 10 & 11 & & 9.0 & II \\
\hline $\mathrm{Na}$ & 128 & 128 & 142 & & 110 & 145 \\
\hline K & 2.8 & 5.0 & 4.8 & & 4.1 & $4 \cdot 3$ \\
\hline $\mathrm{HCO}_{3}$ & 198 & 205 & 201 & & 171 & 195 \\
\hline $\mathrm{CO}_{3}$ & 0 & & 0 & & 0 & 0 \\
\hline $\mathrm{SO}_{4}$ & 85 & 93 & 96 & & 80 & 119 \\
\hline $\mathrm{Cl}$ & 89 & 90 & 90 & & 80 & 115 \\
\hline $\mathrm{F}$ & .9 & & .7 & & .6 & .7 \\
\hline $\mathrm{NO}_{3}$ & 27 & & 26 & & 22 & 25 \\
\hline B & .70 & .60 & .77 & & .55 & 1.2 \\
\hline $\begin{array}{l}\text { Dis. S. } \\
\text { Sum }\end{array}$ & $\begin{array}{c}543 \\
(470)\end{array}$ & $\begin{array}{c}590 \\
(526)\end{array}$ & $\begin{array}{c}548 \\
(497)\end{array}$ & 566 & $\begin{array}{c}573 \\
(4<4)\end{array}$ & $\begin{array}{c}615 \\
(554)\end{array}$ \\
\hline Hardness & (112) & $(136)$ & $(113)$ & & (122) & $(138)$ \\
\hline$\% \mathrm{Na}$ & 71 & 68 & 72 & & 66 & 69 \\
\hline Micromhos & 843 & 871 & 884 & & 775 & 1,010 \\
\hline $\begin{array}{l}\mathrm{pH} \\
\mathrm{o}_{F}\end{array}$ & 7.8 & ${ }_{71}^{8.1}$ & 7.8 & & 7.8 & 8.0 \\
\hline Date & $7-28-55$ & $2-24-53$ & $7-18-55$ & & $7-18-55$ & $7-18-55$ \\
\hline Depth & 350 & 346 & 346 & 300 & 430 & .225 \\
\hline Lab., No. & DWR R-711 & GP & DWR R-710 & & DWR R-71a & DWR R-713 \\
\hline
\end{tabular}

\begin{tabular}{|c|c|c|c|c|c|c|}
\hline & $: 11 / 8-11 D 1:$ & & $1 / 8-20 \overline{H 2}$ & $:$ & $11 / 8$ & $22 E 1$ \\
\hline $\begin{array}{l}\mathrm{SiO}_{2} \\
\mathrm{Fe}\end{array}$ & 66 & & $\begin{array}{r}61 \\
3.6\end{array}$ & & & $\begin{array}{r}.25 \\
.58\end{array}$ \\
\hline $\begin{array}{l}\mathrm{Ca} \\
\mathrm{Mg} \\
\mathrm{Na} \\
\mathrm{K}\end{array}$ & $\begin{array}{c}35 \\
9.8 \\
128 \\
10\end{array}$ & & $\begin{array}{l}40 \\
15 \\
80 \\
10\end{array}$ & $\begin{array}{l}42 \\
15 \\
74 \\
5.5\end{array}$ & & $\begin{array}{c}29 \\
8.9 \\
206 \\
6.2\end{array}$ \\
\hline $\begin{array}{l}\mathrm{HCO}_{3} \\
\mathrm{CO}_{3} \\
\mathrm{SO}_{4} \\
\mathrm{Cl}\end{array}$ & $\begin{array}{r}193 \\
0 \\
93 \\
89\end{array}$ & 62 & $\begin{array}{r}185 \\
0 \\
95 \\
63\end{array}$ & $\begin{array}{r}183 \\
0 \\
96 \\
59\end{array}$ & 212 & $\begin{array}{r}207 \\
0 \\
148 \\
167\end{array}$ \\
\hline $\begin{array}{l}\mathrm{F} \\
\mathrm{NO}_{3} \\
\mathrm{~B}\end{array}$ & .50 & & 1.0 & $10^{.5}$ & & $\begin{array}{r}1.6 \\
14\end{array}$ \\
\hline $\begin{array}{l}\text { Dis. S. } \\
\text { Sum } \\
\text { Hardness } \\
\end{array}$ & $\begin{array}{l}568 \\
(526) \\
(128) \\
\end{array}$ & 185 & $\begin{array}{c}513 \\
(460) \\
(162) \\
\end{array}$ & $\begin{array}{c}462 \\
(393) \\
(167) \\
\end{array}$ & 170 & $\begin{array}{c}709 \\
(709) \\
(109) \\
\end{array}$ \\
\hline $\begin{array}{l}\text { \% } \\
\text { Micromhos } \\
\text { pH } \\
\text { OF } \\
\text { Date } \\
\text { Depth } \\
\text { Lab., No. }\end{array}$ & $\begin{array}{c}69 \\
789 \\
8.0 \\
75 \\
2-24-53 \\
512 \\
\text { GP }\end{array}$ & $\begin{array}{c}683 \\
\\
12-21-51 \\
500 \\
\mathrm{GW}\end{array}$ & $\begin{array}{c}54 \\
671 \\
7 \cdot 7 \\
74 \\
2-24-53 \\
500 \\
\text { GP }\end{array}$ & $\begin{array}{cc}48 \\
886 \\
7.8 \\
\\
10-31-55 \\
500 \\
\text { DWR R-911 }\end{array}$ & $\begin{array}{c}1,460 \\
12-21-51\end{array}$ & $\begin{array}{c}79 \\
1,150 \\
8.0 \\
70 \\
4-2-52 \\
\text { ow } 8533 \\
\end{array}$ \\
\hline
\end{tabular}




\begin{tabular}{|c|c|c|c|c|c|c|}
\hline \multicolumn{2}{|c|}{$11 / 8-26 \pi$} & \multicolumn{3}{|c|}{$: 11 / 8-26 \mathrm{EI}: 11 / 8-31 \mathrm{P1}: 11 / 8-35 \mathrm{NI}:$} & \multicolumn{2}{|c|}{$11 / 9-22 \Omega 1$} \\
\hline$:$ & 25 & 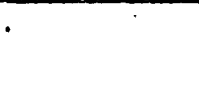 & $\begin{array}{l}58 \\
\text { Trace }\end{array}$ & 0.10 & & $\begin{array}{r}58 \\
.07\end{array}$ \\
\hline & $\begin{array}{r}54 \\
12 \\
216 \\
9.0\end{array}$ & & $\begin{array}{r}120 \\
22 \\
384\end{array}$ & $\begin{array}{l}78 \\
20 \\
44\end{array}$ & & $\begin{array}{l}38 \\
36 \\
82 \\
1.3\end{array}$ \\
\hline & $\begin{array}{r}198 \\
0\end{array}$ & & $\begin{array}{r}317 \\
0\end{array}$ & $\begin{array}{r}207 \\
0\end{array}$ & & 166 \\
\hline 201 & $\begin{array}{l}231 \\
192\end{array}$ & 212 & $\begin{array}{l}293 \\
468\end{array}$ & $\begin{array}{l}497 \\
400\end{array}$ & 72 & $\begin{array}{r}139 \\
87\end{array}$ \\
\hline & 1.3 & & $\begin{array}{l}1.6 \\
8.8 \\
4.0\end{array}$ & 19 & & $14^{.3}$ \\
\hline 195 & $\begin{array}{r}874 \\
(838) \\
(184) \\
\end{array}$ & 125 & $\begin{array}{c}1,660 \\
(1,520) \\
(390) \\
\end{array}$ & $\begin{array}{c}1,160 \\
(1,160) \\
276 \\
\end{array}$ & 120 & $\begin{array}{c}537 \\
(539) \\
243 \\
\end{array}$ \\
\hline 1,400 & $\begin{array}{c}73 \\
1,350 \\
7.7 \\
68\end{array}$ & $\begin{array}{r}1,360 \\
80\end{array}$ & $\begin{array}{c}(68) \\
2,370 \\
7.7\end{array}$ & $\begin{array}{r}(25) \\
1,820 \\
7.4\end{array}$ & 892 & $\begin{array}{c}42 \\
896 \\
7.9 \\
69\end{array}$ \\
\hline $\begin{array}{l}11-27-51 \\
400 \\
G W\end{array}$ & $\begin{array}{c}2-25-53 \\
400 \\
\text { GP }\end{array}$ & $\begin{array}{c}11-27-51 \\
175 \\
\mathrm{GW}\end{array}$ & $\begin{array}{c}9-12-55 \\
303 \\
\text { SE } 408048\end{array}$ & $\begin{array}{c}12-13-56 \\
667 \\
\text { H } 98626 \\
\end{array}$ & $\begin{array}{c}12-21-51 \\
193\end{array}$ & $\begin{array}{c}4-2-52 \\
193 \\
2 W \quad 8527 \\
\end{array}$ \\
\hline
\end{tabular}

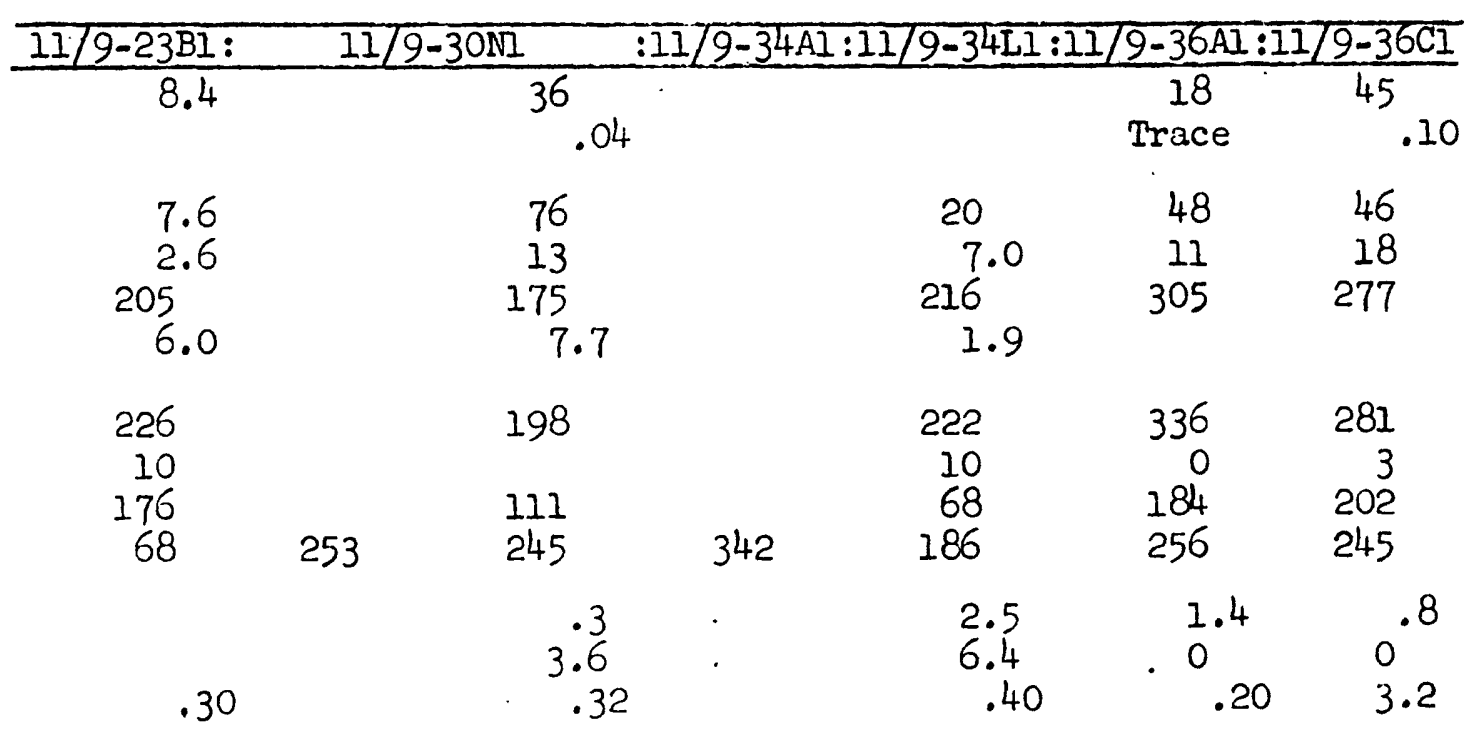

\begin{tabular}{|c|c|c|c|c|c|c|}
\hline $\begin{array}{c}598 \\
(595) \\
(30)\end{array}$ & 270 & $\begin{array}{c}765 \\
(765) \\
243 \\
\end{array}$ & 515 & $\begin{array}{r}648 \\
(628) \\
(79) \\
\end{array}$ & $\begin{array}{r}1,160 \\
(989) \\
(165) \\
\end{array}$ & $\begin{array}{r}1,120 \\
(978) \\
(189) \\
\end{array}$ \\
\hline $\begin{array}{r}94 \\
964 \\
8.8\end{array}$ & 1,320 & $\begin{array}{c}60 \\
1,300 \\
7.7\end{array}$ & 2,100 & $\begin{array}{r}(85) \\
1,170 \\
8.3\end{array}$ & $\begin{array}{c}(80) \\
1,830 \\
8.2\end{array}$ & $(76)$ \\
\hline $\begin{array}{c}66 \\
2-24-53 \\
200 \\
\text { GP }\end{array}$ & $\begin{array}{c}12-21-51 \\
200 \\
\mathrm{GW}\end{array}$ & $\begin{array}{c}68 \\
4-5-52 \\
200 \\
\text { QW } 8532\end{array}$ & $\begin{array}{r}12-21-51 \\
193.5\end{array}$ & $\begin{array}{l}8-23-55 \\
\text { DWR } 6106\end{array}$ & $\begin{array}{c}6-27-55 \\
610 \\
\text { SE } 403956 \\
\end{array}$ & $\begin{array}{c}7-29-55 \\
407 \\
\text { SE } 405710 \\
\end{array}$ \\
\hline
\end{tabular}




\begin{tabular}{|c|c|c|c|c|c|c|}
\hline & \multicolumn{2}{|c|}{$: 11 / 9-36 D 1: 11 / 9-36 \mathrm{HI}:$} & \multicolumn{2}{|c|}{$11 / 10-36 \mathrm{HI}$} & \multicolumn{2}{|c|}{$: 12 / 10-35 \mathrm{PI}: 29 / 39-12 \mathrm{N1}$} \\
\hline $\begin{array}{l}\mathrm{SiO}_{2} \\
\mathrm{Fe}\end{array}$ & $\begin{array}{l}50 \\
.20\end{array}$ & $\begin{array}{c}60 \\
\text { Trace }\end{array}$ & $\begin{array}{l}26 \\
.20\end{array}$ & & 25 & \\
\hline $\begin{array}{l}\mathrm{Ca} \\
\mathrm{Mg} \\
\mathrm{Na} \\
\mathrm{K}\end{array}$ & $\begin{array}{c}32 \\
6.8 \\
222\end{array}$ & $\begin{array}{r}33 \\
11 \\
294\end{array}$ & $\begin{array}{r}90 \\
27 \\
267\end{array}$ & $\begin{array}{c}92 \\
22 \\
260 \\
4.4\end{array}$ & $\begin{array}{c}19 \\
4.0 \\
143 \\
3.0\end{array}$ & $\begin{array}{r}74 \\
60 \\
143 \\
7.3\end{array}$ \\
\hline $\begin{array}{l}\mathrm{HCO}_{3} \\
\mathrm{CO}_{3} \\
\mathrm{SO}_{4} \\
\mathrm{Cl}\end{array}$ & $\begin{array}{r}305 \\
6 \\
132 \\
137\end{array}$ & $\begin{array}{l}305 \\
182 \\
230\end{array}$ & $\begin{array}{l}329 \\
189 \\
320\end{array}$ & $\begin{array}{r}337 \\
0 \\
186 \\
300\end{array}$ & $\begin{array}{r}210 \\
99 \\
70\end{array}$ & $\begin{array}{r}302 \\
0 \\
432 \\
25\end{array}$ \\
\hline $\begin{array}{l}\mathrm{F} \\
\mathrm{NO}_{3} \\
\mathrm{~B}_{3}\end{array}$ & 3.4 & 2.6 & .40 & $0^{.6}$ & $\begin{array}{c}1.6 \\
2.5 \\
.10\end{array}$ & $\begin{array}{r}.7 \\
7.6 \\
.70\end{array}$ \\
\hline $\begin{array}{l}\text { Dis. S. } \\
\text { Sum } \\
\text { Hardness }\end{array}$ & $\begin{array}{r}891 \\
(740) \\
(108) \\
\end{array}$ & $\begin{array}{c}1,110 \\
(965) \\
(128)\end{array}$ & $\begin{array}{c}1,250 \\
(1,080) \\
338\end{array}$ & $\begin{array}{c}1,100 \\
(1,030) \\
(320)\end{array}$ & $\begin{array}{r}(471) \\
(62)\end{array}$ & $\begin{array}{c}992 \\
(899) \\
(432)\end{array}$ \\
\hline $\begin{array}{l}\% \mathrm{Na} \\
\mathrm{Micromhos} \\
\mathrm{pH} \\
\mathrm{O}_{\mathrm{F}}\end{array}$ & $\begin{array}{r}(82) \\
8.3\end{array}$ & $\begin{array}{r}(83) \\
8.1\end{array}$ & $\begin{array}{r}(63) \\
1,700 \\
7.5\end{array}$ & $\begin{array}{c}(63) \\
1,810 \\
7.7\end{array}$ & $\begin{array}{r}82 \\
738 \\
7.5\end{array}$ & $\begin{array}{r}41 \\
1,330 \\
7.4\end{array}$ \\
\hline $\begin{array}{l}\text { Date } \\
\text { Depth } \\
\text { Lab., No. }\end{array}$ & $\begin{array}{c}7-29-55 \\
414 \\
\text { SE } 405709 \\
\end{array}$ & $\begin{array}{c}7-29-55 \\
300 \\
\text { SE } 405711 \\
\end{array}$ & $\begin{array}{c}1-28-53 \\
297\end{array}$ & $\begin{array}{c}2-18-55 \\
297 \\
\text { DWR } 5365\end{array}$ & $\begin{array}{c}5-14-53 \\
378 \\
\text { GP }\end{array}$ & $\begin{array}{c}9-26.55 \\
520 \\
\text { DWR R-869 } \\
\end{array}$ \\
\hline
\end{tabular}

\begin{tabular}{|c|c|c|c|c|c|c|}
\hline \multirow[b]{2}{*}{$\begin{array}{l}\mathrm{SiO}_{2} \\
\mathrm{Fe}\end{array}$} & \multicolumn{2}{|c|}{$: 29 / 39-12 \times 2: 29 / 39-12 \times 3:$} & \multicolumn{2}{|c|}{$29 / 39-15 \mathrm{M}$} & \multicolumn{2}{|c|}{$: 29 / 39-29 \mathrm{ML}: 29 / 39-29 \mathrm{NI}$} \\
\hline & $\begin{array}{l}55 \\
.15\end{array}$ & $7^{74} .09$ & & 20 & 4.0 & \\
\hline $\begin{array}{l}\mathrm{Ca} \\
\mathrm{Mg} \\
\mathrm{Na} \\
\mathrm{K}\end{array}$ & $\begin{array}{r}99 \\
52 \\
\text { a. } 54\end{array}$ & $\begin{array}{c}32 \\
9.7 \\
a(67)^{7}\end{array}$ & $\begin{array}{r}79 \\
117 \\
182 \\
11\end{array}$ & $\begin{array}{r}144 \\
102 \\
184 \\
13\end{array}$ & $\begin{array}{r}50 \\
37 \\
141 \\
9.0\end{array}$ & $\begin{array}{r}49 \\
53 \\
132 \\
12\end{array}$ \\
\hline $\begin{array}{l}\mathrm{HCO}_{3} \\
\mathrm{CO}_{3} \\
\mathrm{SO}_{4} \\
\mathrm{Cl}\end{array}$ & $\begin{array}{r}285 \\
0 \\
468 \\
21\end{array}$ & $\begin{array}{r}176 \\
10 \\
59 \\
27\end{array}$ & $\begin{array}{r}216 \\
4 \\
782 \\
50\end{array}$ & $\begin{array}{r}0 \\
0 \\
810 \\
260\end{array}$ & $\begin{array}{l}227 \\
138 \\
187\end{array}$ & $\begin{array}{r}285 \\
17 \\
225 \\
100\end{array}$ \\
\hline $\begin{array}{l}\mathrm{F} \\
\mathrm{NO}_{3} \\
\mathrm{~B}\end{array}$ & .8 & 6.4 & $\begin{array}{l}.7 \\
3.7 \\
.60\end{array}$ & $\begin{array}{l}.4 \\
.75\end{array}$ & $\begin{array}{l}.8 \\
5.0 \\
1.3\end{array}$ & $\begin{array}{l}1.0 \\
8.4 \\
1.6\end{array}$ \\
\hline $\begin{array}{l}\text { Dis. S. } \\
\text { Sum } \\
\text { Hardness }\end{array}$ & $\begin{array}{l}1,010 \\
\left(\begin{array}{l}990) \\
(461)\end{array}\right.\end{array}$ & $\begin{array}{l}387 \\
(372) \\
(120)\end{array}$ & $\begin{array}{r}1,400 \\
(1,340) \\
(679) \\
\end{array}$ & $\begin{array}{r}1,510 \\
(1,530) \\
(779) \\
\end{array}$ & $\begin{array}{r}714 \\
(685) \\
(277) \\
\end{array}$ & $\begin{array}{r}783 \\
(739) \\
(341) \\
\end{array}$ \\
\hline $\begin{array}{l}\text { \% Na } \\
\text { Micromhos } \\
\text { pH } \\
\mathrm{O}_{\mathrm{F}}\end{array}$ & (42) & $(55)$ & $\begin{array}{r}(36) \\
1,840 \\
8.4\end{array}$ & $\begin{array}{c}(33) \\
2,280 \\
4.0\end{array}$ & $\begin{array}{c}(52) \\
1,130 \\
7.7 \\
76\end{array}$ & $\begin{array}{r}(45) \\
1,140 \\
8.3\end{array}$ \\
\hline $\begin{array}{l}\text { Date } \\
\text { Depth } \\
\text { Lab., No. }\end{array}$ & $\begin{array}{c}10-2-17 \\
520 \\
\text { DGT } \\
\end{array}$ & $\begin{array}{c}10-5-17 \\
1,400 \\
\text { DGT }\end{array}$ & $\begin{array}{r}6-5-53 \\
\text { DWR P-675 } \\
\end{array}$ & $\begin{array}{r}7-1-57 \\
80 \\
\text { DWR T-864 } \\
\end{array}$ & $\begin{array}{c}4-23-53 \\
265 \\
\mathrm{GP} \\
\end{array}$ & $\begin{array}{r}8-24-55 \\
165 \\
\text { DWR } 6084 \\
\end{array}$ \\
\hline
\end{tabular}




$\begin{array}{rrrrrrr}48 & 47 & 8.0 & 53 & 164 & 66 & 64 \\ 38 & 36 & 2.0 & 3.2 & 4.0 & 12 & 16 \\ 174 & 160 & 115 & 734 & 602 & 102 & 106 \\ 8.0 & .8 & 2.0 & 6.0 & 4.0 & 2.6 & 3.5 \\ 235 & 146 & 165 & 196 & 102 & 256 & 281 \\ 0 & 0 & 0 & & 0 & 2 & 0 \\ 104 & 74 & 79 & 934 & 999 & 156 & 155 \\ 230 & 305 & 39 & 427 & 480 & 42 & 43\end{array}$

1.2

3.7

2

2.2

6.9

.6

1.0

8.0

4.0

.81 .5

2.2

2.0

.7

3.9

4.7

.38

33.4

1.8

1.7

2.0

\begin{tabular}{ccccccc}
849 & 1,030 & 349 & & & 563 & 588 \\
$(759)$ & $(763)$ & $(330)$ & $(2,350)$ & $(2,340)$ & $(515)$ & $(561)$ \\
$(276)$ & $(266)$ & $(28)$ & $(146)$ & $(426)$ & $(214)$ & $(226)$ \\
\hline$(57)$ & $(57)$ & 89 & $(91)$ & $(75)$ & $(51)$ & $(50)$ \\
1,320 & 1,440 & 570 & 3,480 & 3,500 & 822 & 931
\end{tabular}

7.9

7.9

7.5

78

78

7-1-57

$8-24-55$

165

460

DWR T-883 DWR 6109 $\begin{array}{ccccc}8-24-55 & 10-7-53 & 3-11-53 & 8-24-55 & 7-1-57\end{array}$ DWR R-913 GP GP DWR 17827 DWR T-885

\begin{tabular}{rrrrrcc}
\hline & \multicolumn{3}{c}{$30 / 37-23 \mathrm{~J} 3$} & \multicolumn{3}{c}{$: 30 / 37-24 \mathrm{B1}: 30 / 37-24 \mathrm{KI}$} \\
\hline $\mathrm{b} 32$ & 40 & $\mathrm{~b} 38$ & 41 & 63 & 32 & 50 \\
& & & & & Trace & \\
65 & 72 & 71 & 73 & 176 & 65 & 71 \\
14 & 14 & 14 & 14 & 22 & 14 & 7.0 \\
90 & 100 & 98 & 85 & 296 & 90 & 113 \\
& & & & & & 4.0 \\
294 & 316 & 303 & 285 & 276 & 294 & 275 \\
116 & 125 & 128 & 114 & 145 & 116 & 174 \\
37 & 48 & 47 & 53 & 565 & 37 & 44 \\
& & & & & & \\
& & & & 3.6 & & 2.6 \\
& & & & & &
\end{tabular}

\begin{tabular}{ccccccc}
$(499)$ & 555 & 546 & 521 & 1,410 & 499 & \\
$(499)$ & $(555)$ & $(545)$ & $(520)$ & $(1,410)$ & $(499)$ & $(605)$ \\
$(220)$ & 240 & 236 & 242 & 531 & 220 & $(206)$ \\
\hline$(47)$ & $(48)$ & $(48)$ & $(43)$ & $(55)$ & $(47)$ & 54 \\
& & & & & & 871
\end{tabular}

$$
\begin{array}{llll}
7.6 & 7.3 & 7.7 & 6.7
\end{array}
$$

7.7

$\begin{array}{lcccccc}6-7-09 & 1-29-49 & 8-17-49 & 4-3-51 & 10-31-52 & 6-7-09 & 5-13-53 \\ 4331 & 431 & 431 & 431 & & \end{array}$ 431 SP W-9279 SP W-9415 SP W-9631 SP W-9948 CW

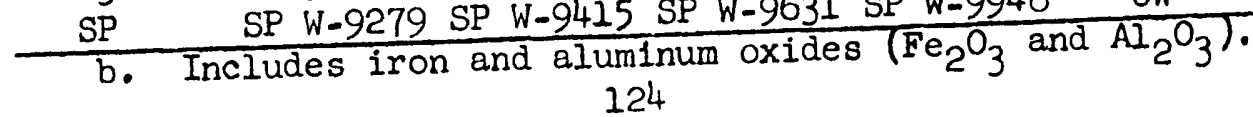




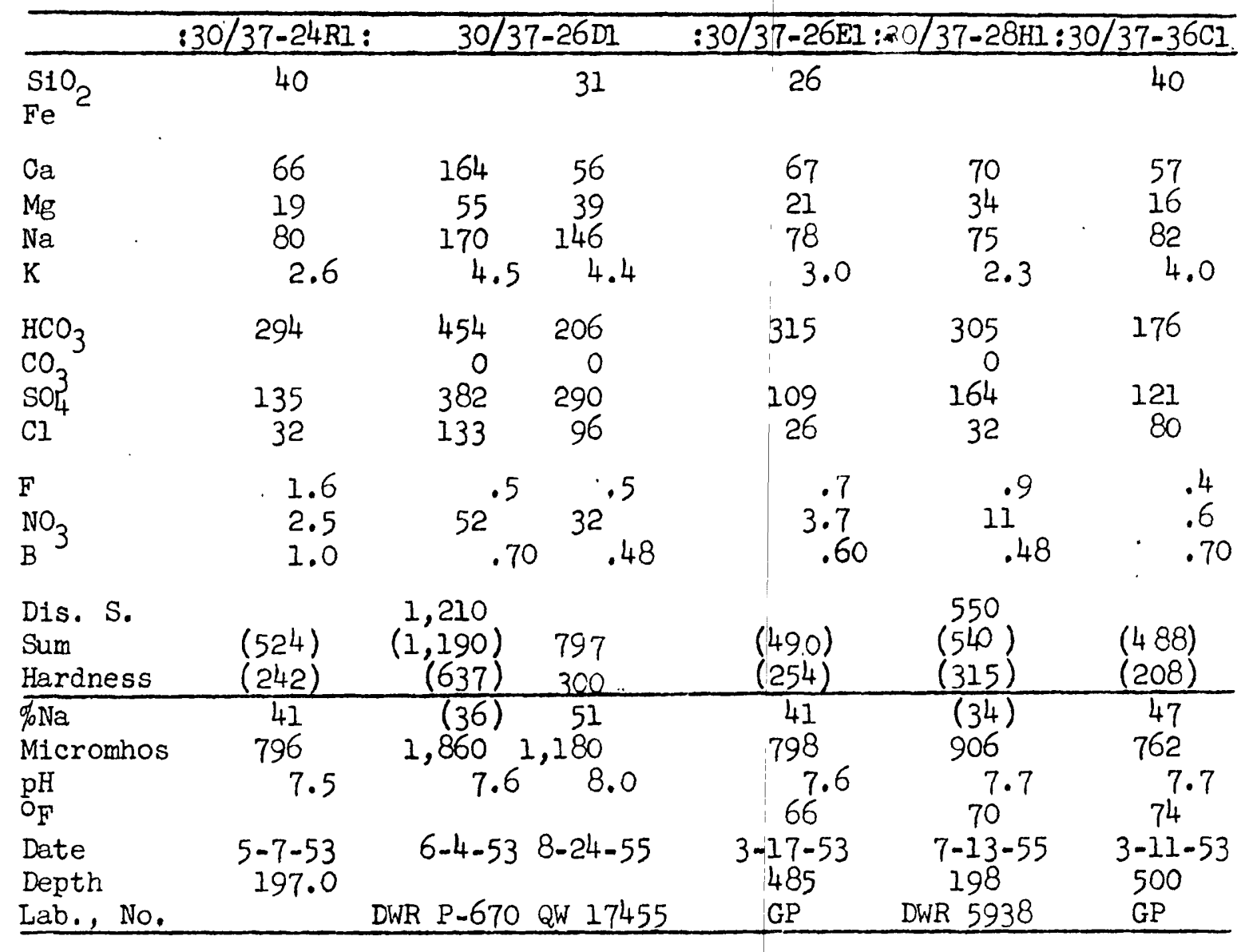

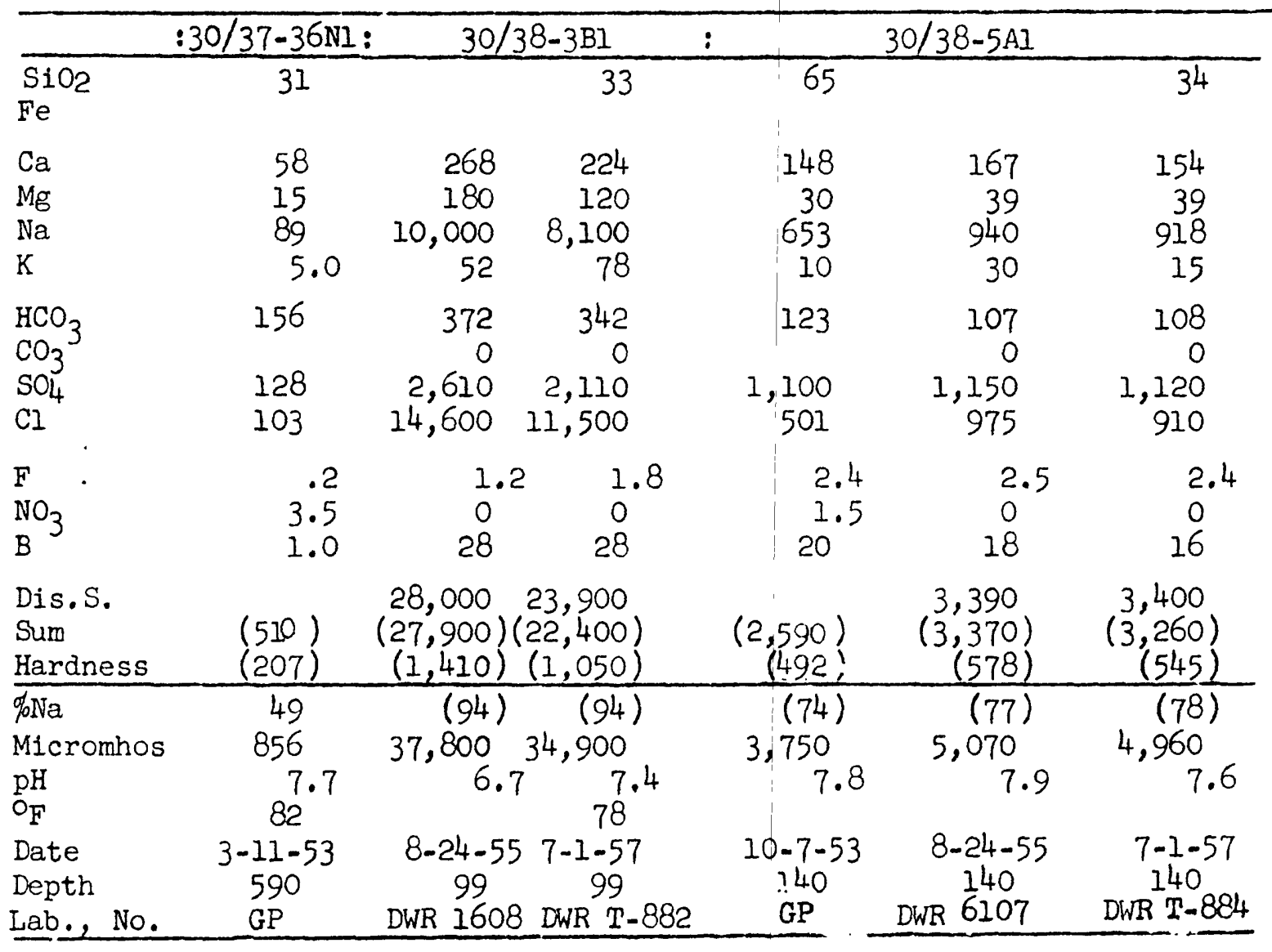




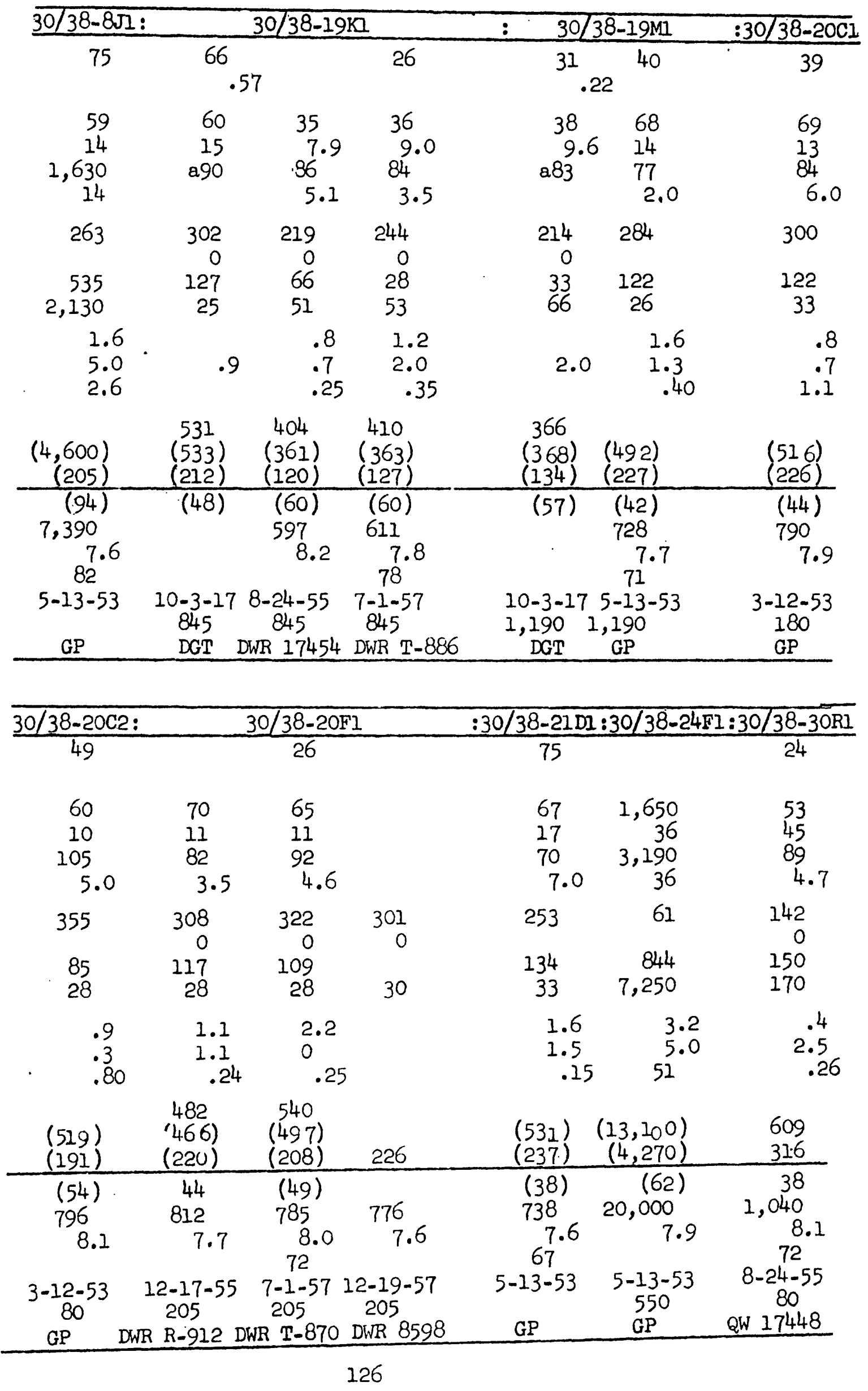




\begin{tabular}{|c|c|c|c|c|c|c|}
\hline & \multicolumn{4}{|c|}{$: 30 / 38-30 \mathrm{RL}: 30 / 38-3201: 30 / 38-34 \mathrm{Cl}: 30 / 39-8 \mathrm{Al}:$} & \multicolumn{2}{|c|}{$31 / 37-1 \mathrm{HL}$} \\
\hline $\begin{array}{l}\mathrm{SiO}_{2} \\
\mathrm{Fe}\end{array}$ & 19 & 30 & 20 & 2.5 & & 20 \\
\hline $\mathrm{Ca}$ & $\begin{array}{c}100 \\
30 \\
98 \\
3.9\end{array}$ & $\begin{array}{r}837 \\
370 \\
589 \\
10\end{array}$ & $\begin{array}{c}355 \\
9.8 \\
78 \\
4.0\end{array}$ & $\begin{array}{r}145 \\
36 \\
463 \\
13\end{array}$ & $\begin{array}{c}62 \\
14 \\
75 \\
3.9\end{array}$ & $\begin{array}{l}55 \\
17 \\
81 \\
4.2\end{array}$ \\
\hline $\begin{array}{l}\mathrm{HCO}_{3} \\
\mathrm{CO}_{3}{ }^{3} \\
\mathrm{SO}_{4} \\
\mathrm{Cl}^{4}\end{array}$ & $\begin{array}{r}172 \\
0 \\
163 \\
215\end{array}$ & $\begin{array}{r}155 \\
0 \\
1,660 \\
2,120\end{array}$ & $\begin{array}{r}107 \\
0 \\
834 \\
92\end{array}$ & $\begin{array}{l}151 \\
303 \\
778\end{array}$ & $\begin{array}{r}146 \\
0 \\
136 \\
81\end{array}$ & $\begin{array}{r}146 \\
0 \\
135 \\
85\end{array}$ \\
\hline $\begin{array}{l}\mathrm{F} \\
\mathrm{NO}_{3} \\
\mathrm{~B}\end{array}$ & $\begin{array}{l}1.4 \\
0 \\
.35\end{array}$ & $\begin{array}{r}.4 \\
5.0 \\
3.0\end{array}$ & $\begin{array}{l}.4 \\
3.0 \\
.85\end{array}$ & $\begin{array}{r}.4 \\
5.0 \\
4.6\end{array}$ & $\begin{array}{r}.2 \\
4.6 \\
.33\end{array}$ & $\begin{array}{c}.3 \\
5.3 \\
.21\end{array}$ \\
\hline $\begin{array}{l}\text { Dis. S. } \\
\text { Sum } \\
\text { Hardness }\end{array}$ & $\begin{array}{c}775 \\
(715) \\
(373)\end{array}$ & $\begin{array}{l}5,700) \\
(3,610) \\
\end{array}$ & $\begin{array}{r}(1,450) \\
(927)\end{array}$ & $\begin{array}{r}(1,820) \\
\quad(510) \\
\end{array}$ & $\begin{array}{r}477 \\
(449) \\
(212) \\
\end{array}$ & $\begin{array}{r}482 \\
(475) \\
(207) \\
\end{array}$ \\
\hline $\begin{array}{l}\% \mathrm{Na} \\
\text { Micromhos } \\
\mathrm{pH}\end{array}$ & $\begin{array}{c}(36) \\
1,240 \\
8.2\end{array}$ & $\begin{array}{c}(26) \\
7,900 \\
7.3\end{array}$ & $\begin{array}{c}(15) \\
1,890 \\
7.6\end{array}$ & $\begin{array}{c}(66) \\
3,080 \\
7.6\end{array}$ & $\begin{array}{r}(43) \\
8.2\end{array}$ & $\begin{array}{c}(46) \\
776 \\
8.2\end{array}$ \\
\hline & $76^{8.2}$ & & $82^{10}$ & & 79. & \\
\hline $\begin{array}{l}\text { Date } \\
\text { Depth } \\
\text { Lab., No. }\end{array}$ & $\begin{array}{c}7-1-57 \\
80 \\
\text { DWR T-871 }\end{array}$ & $\begin{array}{c}5-13-53 \\
300 \\
\text { GP }\end{array}$ & $\begin{array}{c}5-13-53 \\
367 \\
\text { GP }\end{array}$ & $\begin{array}{c}5-13-53 \\
268.3 \\
\text { GP }\end{array}$ & $\begin{array}{c}8-24-55 \\
504 \\
\text { DWR } 17786\end{array}$ & $\begin{array}{c}7-1-57 \\
504 \\
\text { DWR T-888 }\end{array}$ \\
\hline
\end{tabular}

\begin{tabular}{|c|c|c|c|c|c|c|}
\hline & $: 31 / 37-5 \mathrm{M}:$ & $31 / 37-$ & $-10 \mathrm{AI}$ & $31 / 37$ & $-14 L 1$ & $: 31 / 37-35 \mathrm{NI}$ \\
\hline $\begin{array}{l}\mathrm{SiO}_{2} \\
\mathrm{Fe}\end{array}$ & & & 23 & 26 & & 30 \\
\hline $\begin{array}{l}\mathrm{Ca} \\
\mathrm{Mg} \\
\mathrm{Na} \\
\mathrm{K}\end{array}$ & $\begin{array}{r}43 \\
34 \\
108 \\
4.4\end{array}$ & $\begin{array}{l}63 \\
12 \\
81 \\
4.7\end{array}$ & $\begin{array}{l}52 \\
19 \\
90 \\
4.6\end{array}$ & $\begin{array}{l}62 \\
14 \\
79 \\
4.0\end{array}$ & $\begin{array}{l}65 \\
16 \\
80 \\
4.3\end{array}$ & $\begin{array}{r}62 \\
14 \\
112 \\
3.0\end{array}$ \\
\hline $\begin{array}{l}\mathrm{HCO}_{3} \\
\mathrm{CO}_{3} \\
\mathrm{SO}_{4} \\
\mathrm{Cl}\end{array}$ & $\begin{array}{r}232 \\
0 \\
240 \\
38\end{array}$ & $\begin{array}{r}151 \\
0 \\
159 \\
73\end{array}$ & $\begin{array}{r}151 \\
0 \\
149 \\
80\end{array}$ & $\begin{array}{r}164 \\
119 \\
88\end{array}$ & $\begin{array}{c}151 \\
2.1 \\
126 \\
96\end{array}$ & $\begin{array}{l}129 \\
123 \\
158\end{array}$ \\
\hline $\begin{array}{l}\mathrm{F} \\
\mathrm{NO}_{3}\end{array}$ & $\begin{array}{l}.7 \\
6.0 \\
1.2\end{array}$ & $\begin{array}{c}.2 \\
5.6 \\
.43\end{array}$ & $\begin{array}{l}.3 \\
6.2 \\
.32\end{array}$ & $\begin{array}{r}.1 \\
1.0 \\
.80\end{array}$ & $\begin{array}{l}.3 \\
4.7 \\
.38\end{array}$ & $\begin{array}{l}.3 \\
2.9 \\
1.0\end{array}$ \\
\hline $\begin{array}{l}\text { Dis. S. } \\
\text { Sum } \\
\text { Hardness }\end{array}$ & $\begin{array}{c}605 \\
(590) \\
(247)\end{array}$ & $\begin{array}{c}504 \\
(473) \\
(207)\end{array}$ & $\begin{array}{c}510 \\
(499) \\
(208) \\
\end{array}$ & $\begin{array}{r}(475) \\
(212) \\
\end{array}$ & $\begin{array}{l}500 \\
(469) \\
(228) \\
\end{array}$ & $\begin{array}{r}(570) \\
(212) \\
\end{array}$ \\
\hline $\begin{array}{l}\text { \%Na } \\
\text { Micromhos } \\
\text { pH } \\
\mathrm{O}_{\mathrm{F}} \\
\text { Date } \\
\text { Depth } \\
\text { Lab., No. } \\
\end{array}$ & $\begin{array}{c}(48) \\
922 \\
7.7 \\
76 \\
7-13-55 \\
205 \\
\text { DIR } 5940\end{array}$ & $\begin{array}{c}(45) \\
788 \\
8.2 \\
80 \\
8-24-55 \\
320 \\
\text { DWR } 17785 \\
\end{array}$ & $\begin{array}{c}(48) \\
802 \\
8.1 \\
80 \\
7-1-57 \\
320 \\
\text { DWR T-889 }\end{array}$ & $\begin{array}{c}(44) \\
783 \\
7.6 \\
72 \\
3-10-53 \\
170.0 \\
\text { GP. }\end{array}$ & $\begin{array}{c}43 \\
802 \\
8.4 \\
\\
7-13-55 \\
170.0 \\
\text { DWR R- }-751\end{array}$ & $\begin{array}{c}(53) \\
1,010 \\
7.9 \\
78 \\
3-10-53 \\
439 \\
\text { GP }\end{array}$ \\
\hline
\end{tabular}




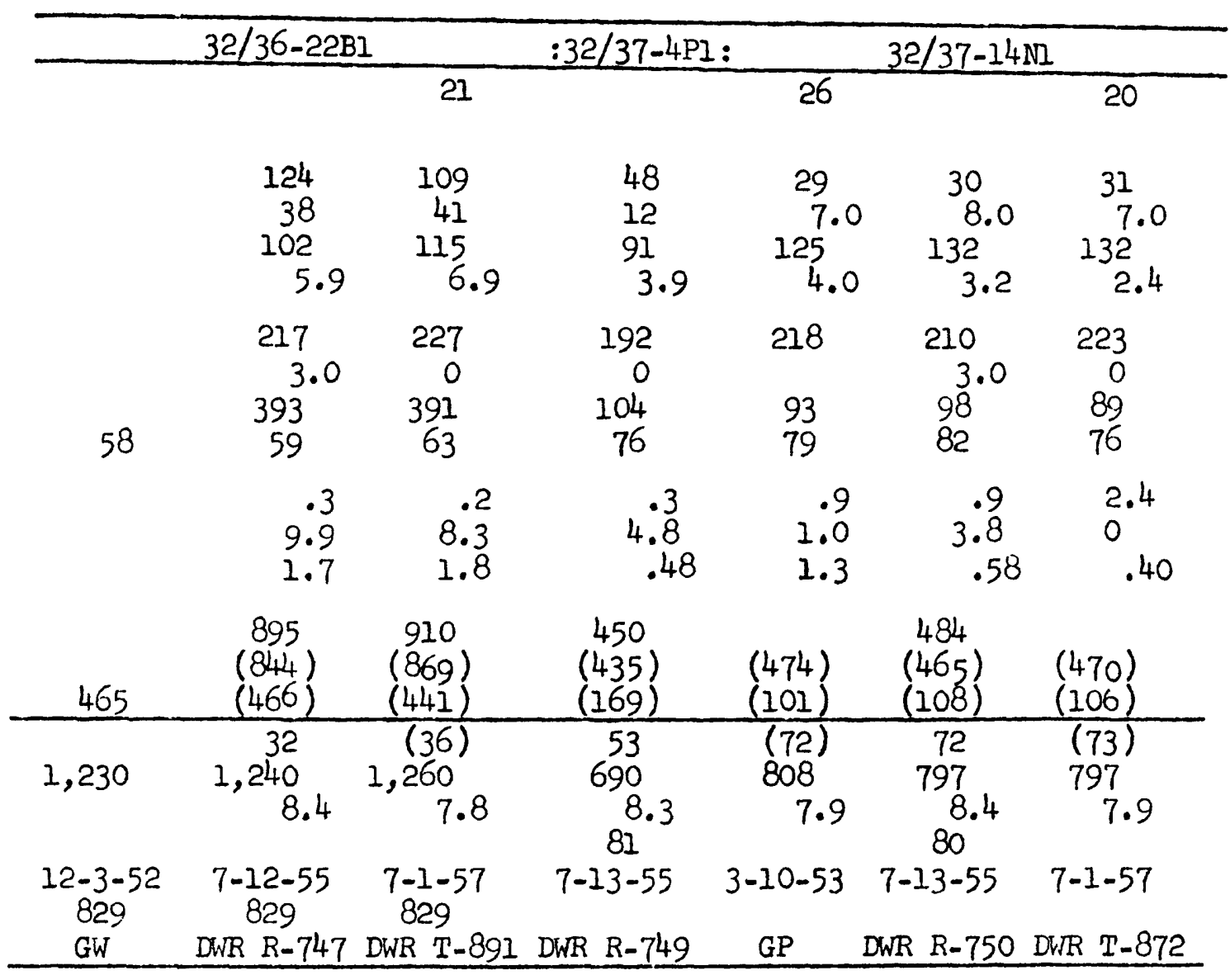

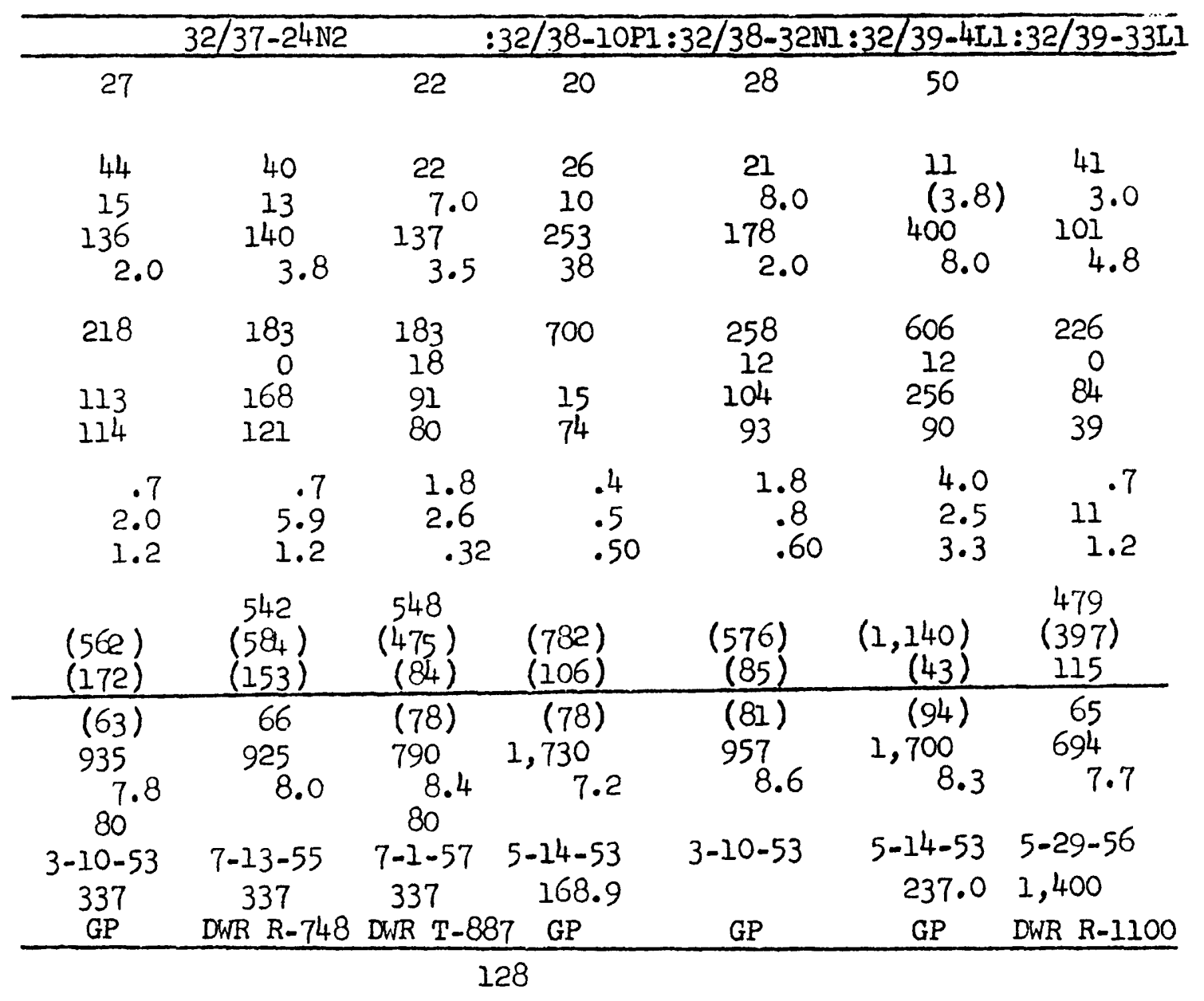

